\title{
Catalytic Enantioselective Synthesis of Aryl-Methyl Organophosphorus Compounds
}

\author{
Arun Yadav, ${ }^{\mathrm{a}, \mathrm{b}}$ Dileep Kumar, ${ }^{\mathrm{a}}$ Manish Kumar Mishra, ${ }^{\mathrm{c}}$ Deeksha ${ }^{\mathrm{a}}$ and Chandra Bhushan Tripathiª, b \\ ${ }^{a}$ Medicinal \& Process Chemistry Division, CSIR-Central Drug Research Institute, Lucknow-226031, India. \\ ${ }^{\mathrm{b}}$ Chemical Sciences Division, Academy of Scientific and Innovative Research (AcSIR), Ghaziabad-201002, India. \\ ${ }^{c}$ Department of Pharmaceutics, College of Pharmacy, University of Minnesota, USA. \\ chandra.tripathi@,cdri.res.in
}

\section{SUPPORTING INFORMATION}

\section{Table of Contents}

A. Effect of solvent and concentration on the reaction $\quad$ S2

$\begin{array}{ll}\text { B. Catalyst screening with } \alpha \text {-naphthol } & \text { S3 }\end{array}$

$\begin{array}{ll}\text { C. Spectral data for newly synthesized compounds } & \text { S3 }\end{array}$

D. Details of Single Crystal $X$-ray Diffraction Analysis of 3z $\quad$ S104 
Solvent Screening:

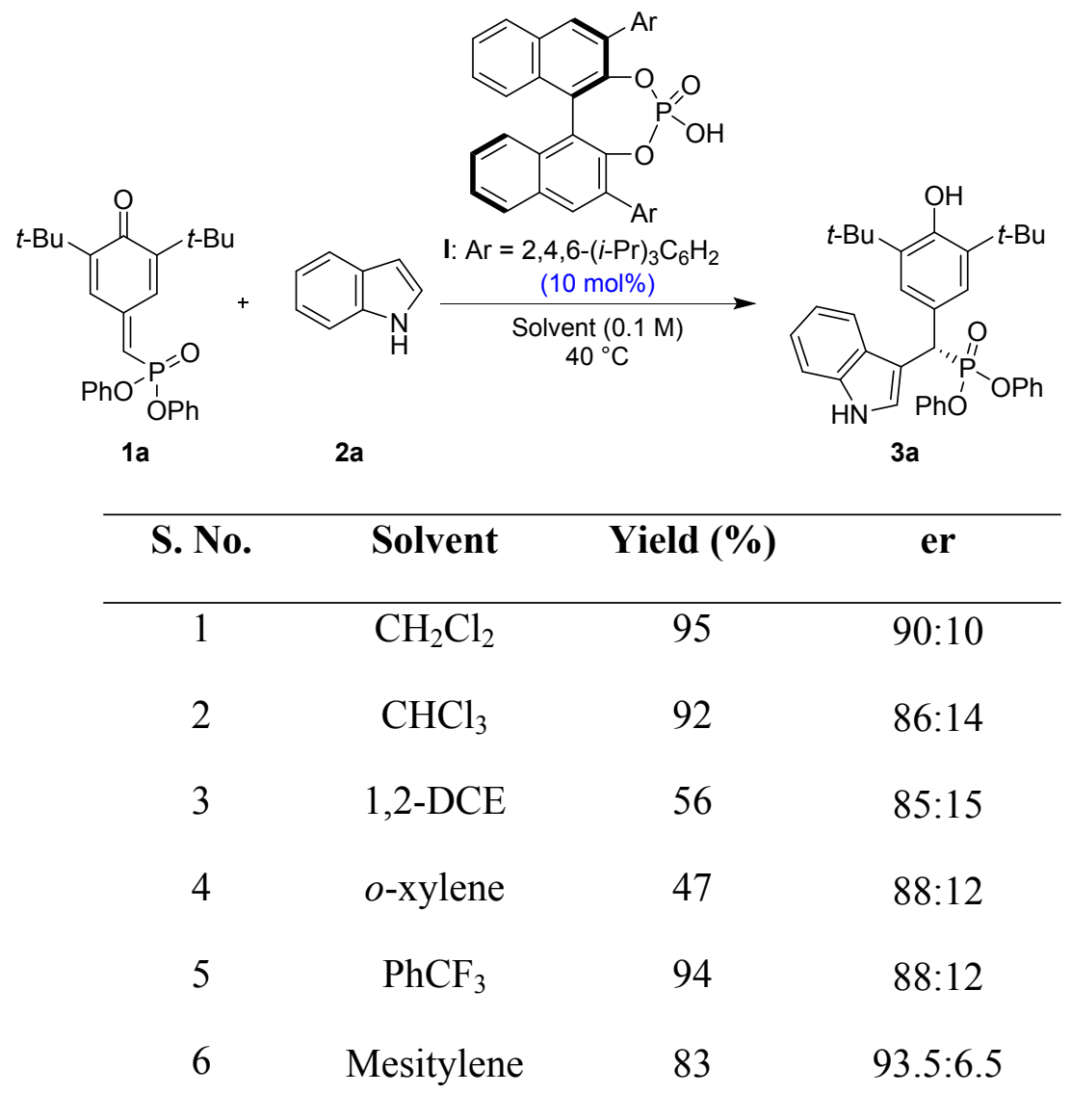

\section{Effect of concentration:}

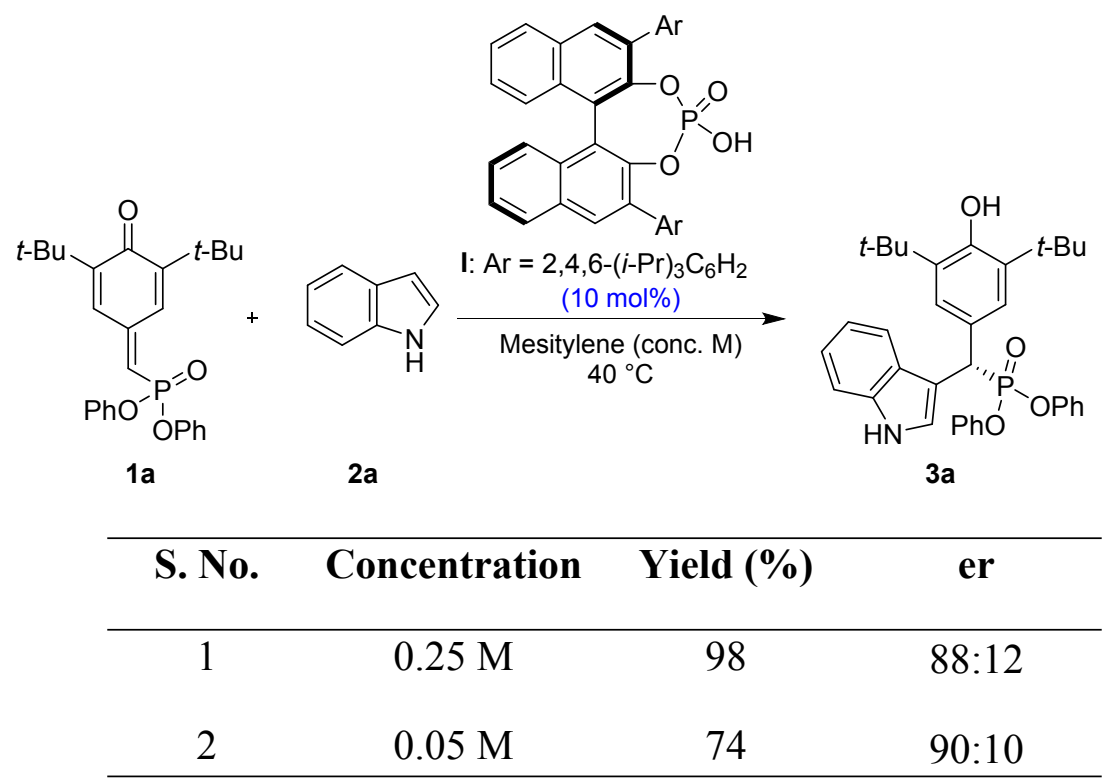




\section{Catalyst screening with $\alpha$-naphthol:}

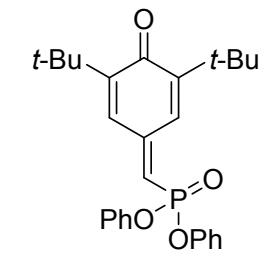

1 a

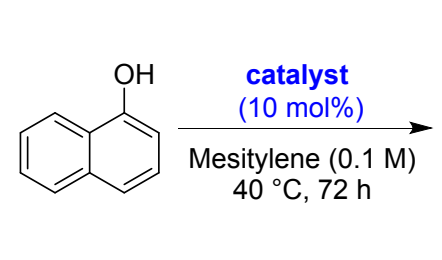

6

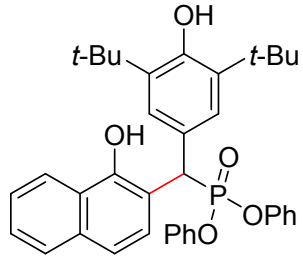

\section{7}

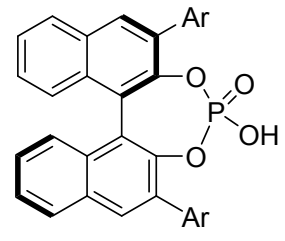

I: $\mathrm{Ar}=2,4,6-(i-\mathrm{Pr})_{3} \mathrm{C}_{6} \mathrm{H}_{2}$ (52\% yield, $77: 23$ er)

II: $\mathrm{Ar}=2,4,6-(c-\mathrm{Hex})_{3} \mathrm{C}_{6} \mathrm{H}_{2}$ (45\% yield, $\left.65: 35 \mathrm{er}\right)$

III: $\mathrm{Ar}=\mathrm{Ph}(51 \%$ yield, $68: 32 \mathrm{er})$

IV: $\mathrm{Ar}=3,5-\left(\mathrm{CF}_{3}\right)_{2} \mathrm{C}_{6} \mathrm{H}_{3}$ (44\% yield, 52: $48 \mathrm{er}$ )

V: $\mathrm{Ar}=\mathrm{SiPh}_{3}(12 \%$ yield, 51: $49 \mathrm{er})$

\section{Spectral data for newly synthesized compounds:}
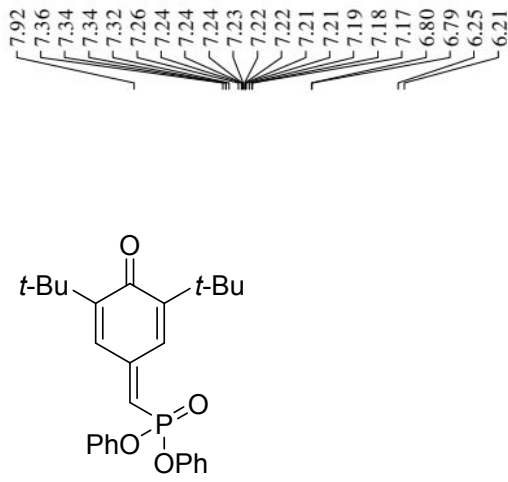

$1 \mathrm{a}$

${ }^{1} \mathrm{H}-\mathrm{NMR}\left(400 \mathrm{MHz}, \mathrm{CDCl}_{3}\right)$
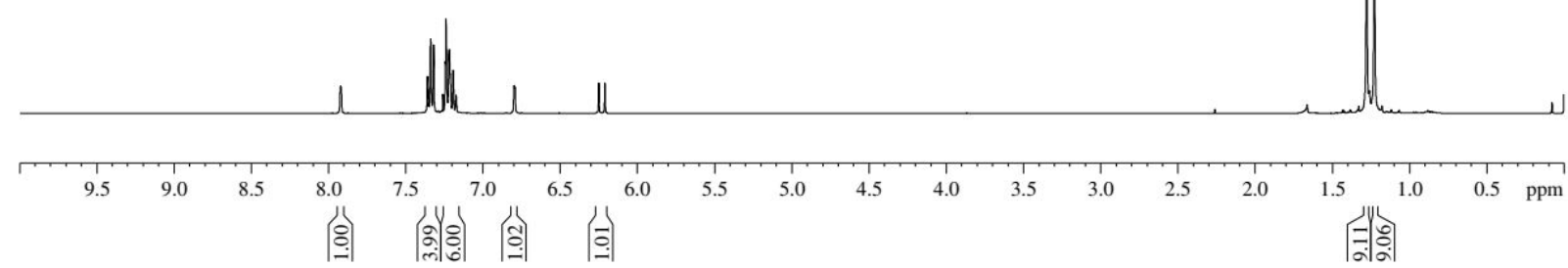

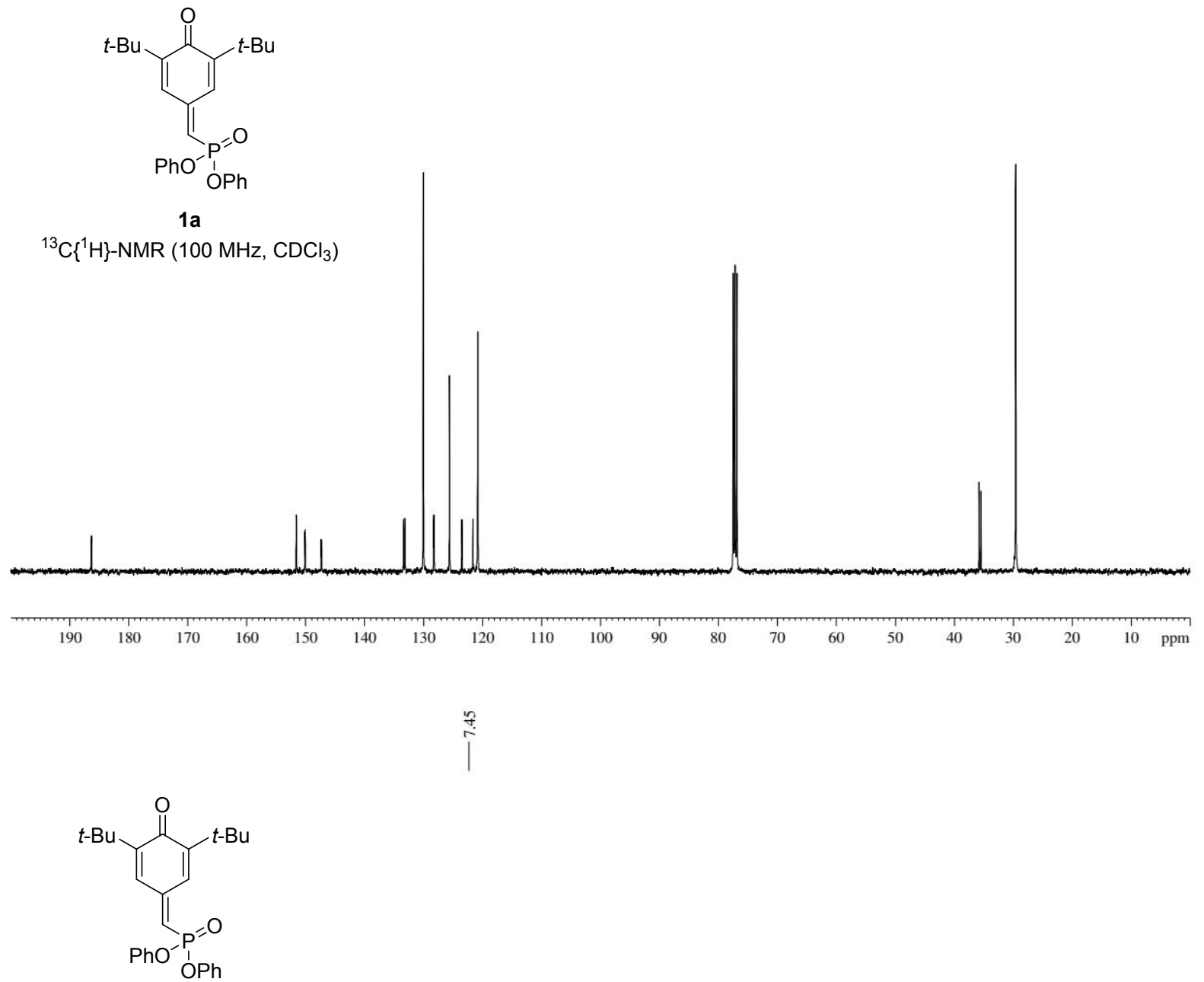

${ }^{31} \mathrm{P}-\mathrm{NMR}\left(162 \mathrm{MHz}, \mathrm{CDCl}_{3}\right.$ )

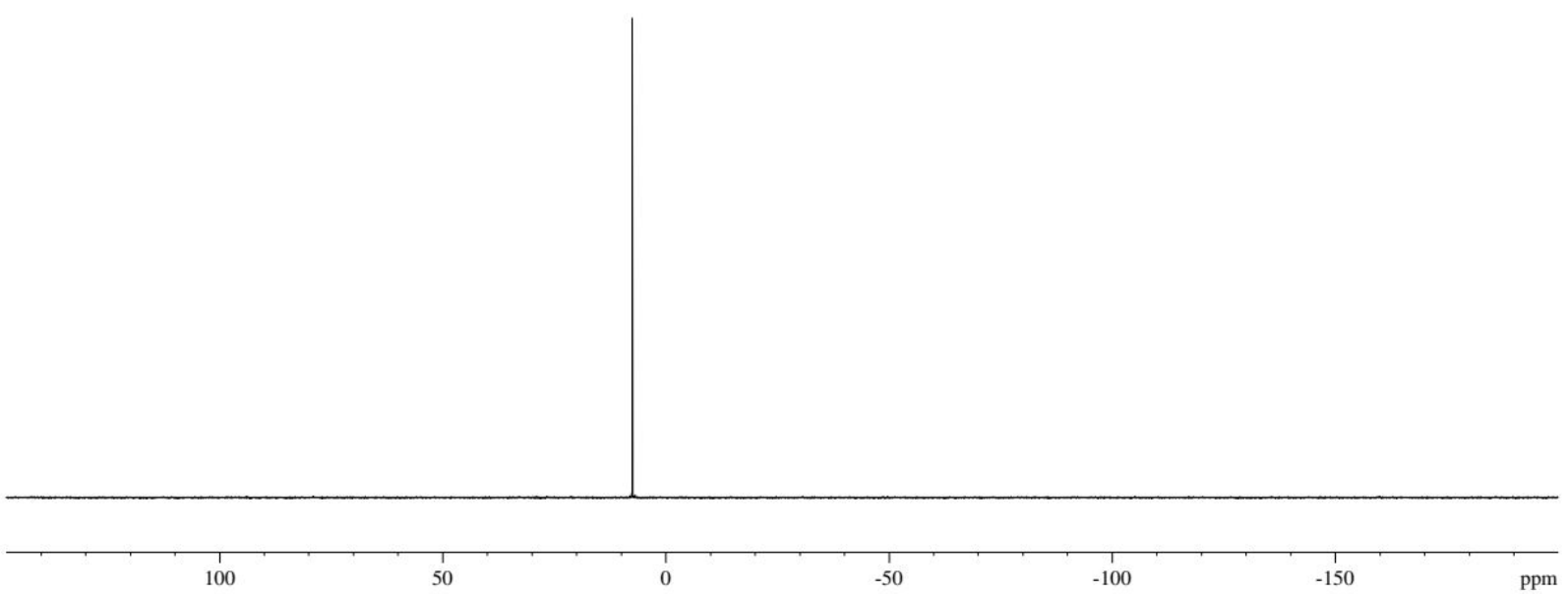




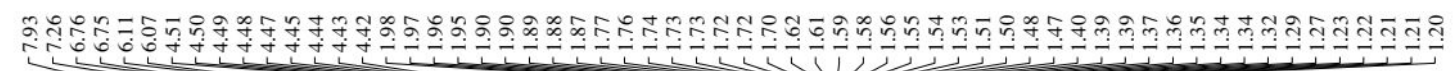<smiles>CCCOP(=O)(C=C1C=C(C(C)(C)C)C(=O)C(C(C)(C)C)=C1)OCC</smiles>

$1 \mathrm{~b}$

${ }^{1} \mathrm{H}-\mathrm{NMR}\left(400 \mathrm{MHz}, \mathrm{CDCl}_{3}\right)$

l.<smiles>CCCCOP(=O)(OC=C1C=C(C(C)(C)C)C(=O)C(C(C)(C)C)=C1)OCCCC</smiles>

$1 b$

${ }^{13} \mathrm{C}\left\{{ }^{1} \mathrm{H}\right\}-\mathrm{NMR}\left(100 \mathrm{MHz}, \mathrm{CDCl}_{3}\right)$
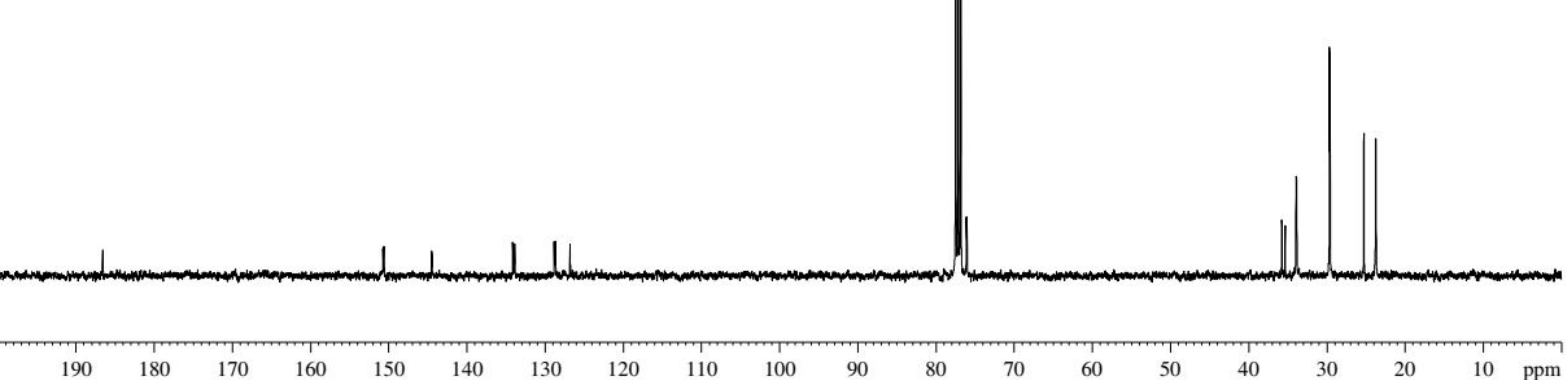


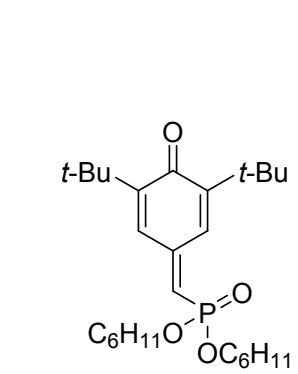

in

$1 \mathrm{~b}$

${ }^{31} \mathrm{P}-\mathrm{NMR}\left(162 \mathrm{MHz}, \mathrm{CDCl}_{3}\right)$

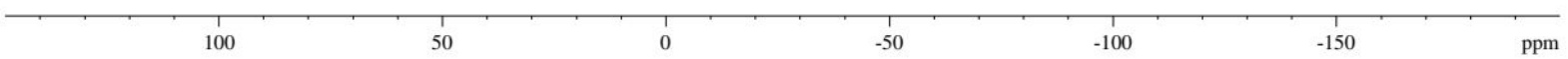
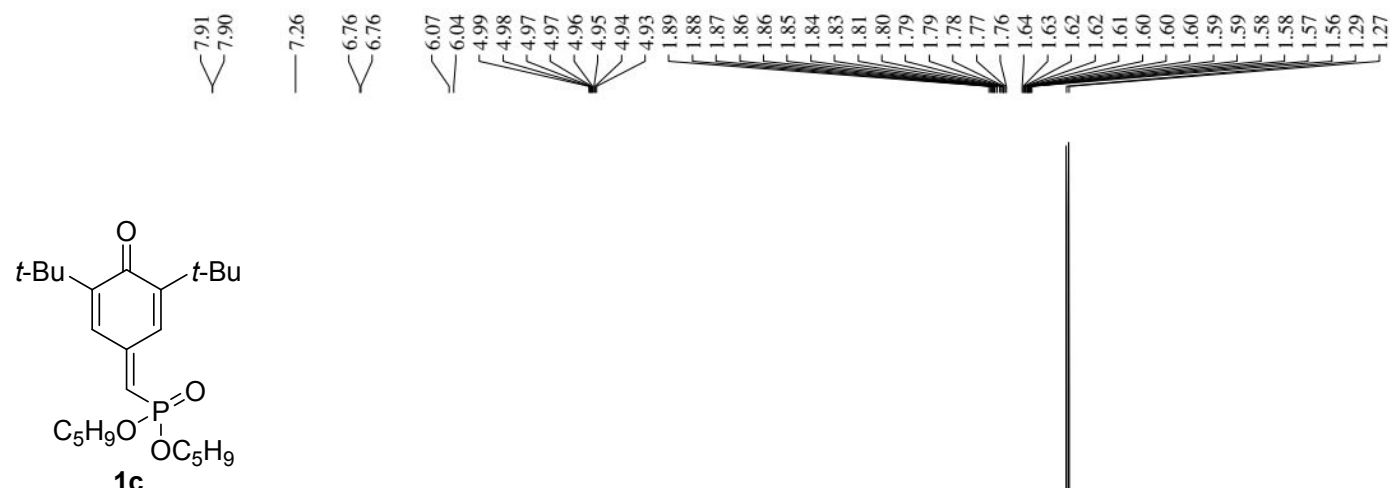

${ }^{1} \mathrm{H}-\mathrm{NMR}\left(400 \mathrm{MHz}, \mathrm{CDCl}_{3}\right)$

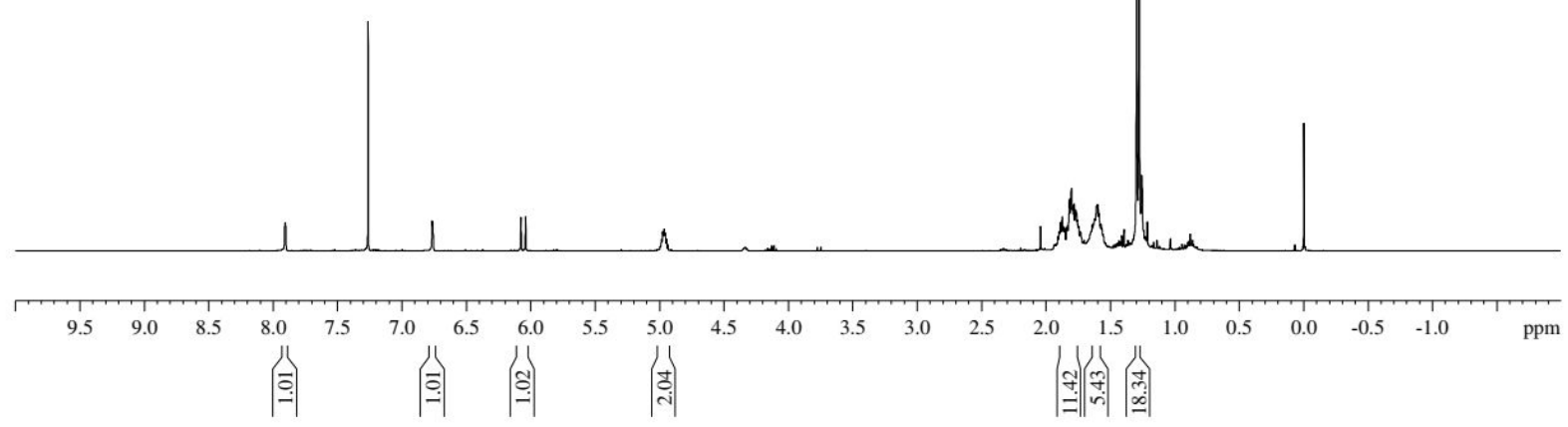



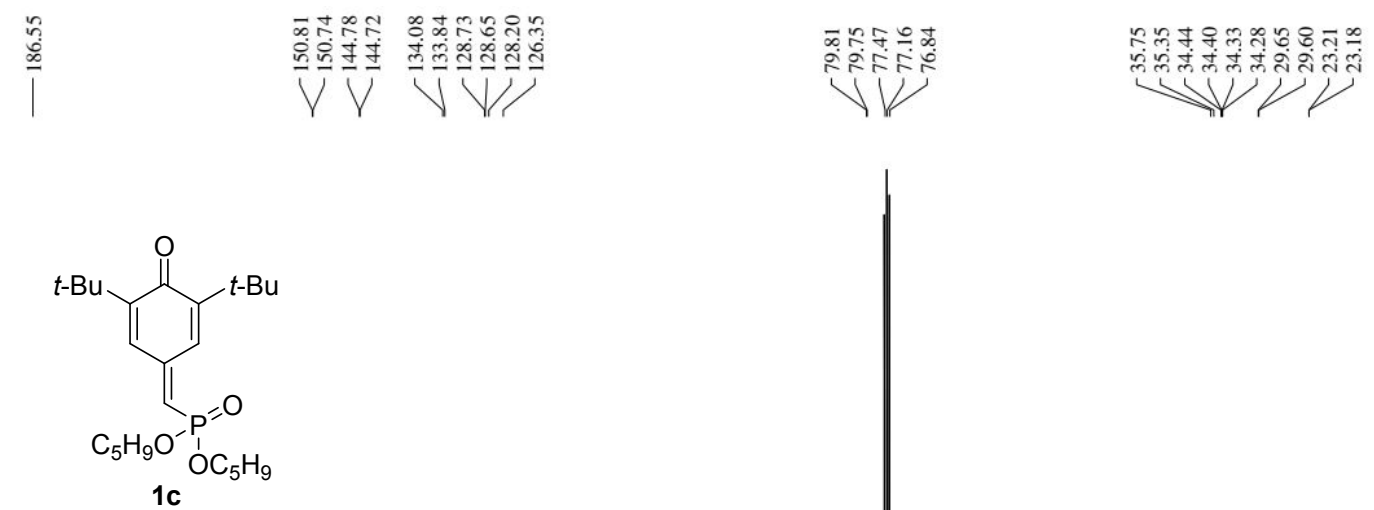

${ }^{13} \mathrm{C}\left\{{ }^{1} \mathrm{H}\right\}-\mathrm{NMR}\left(100 \mathrm{MHz}, \mathrm{CDCl}_{3}\right)$
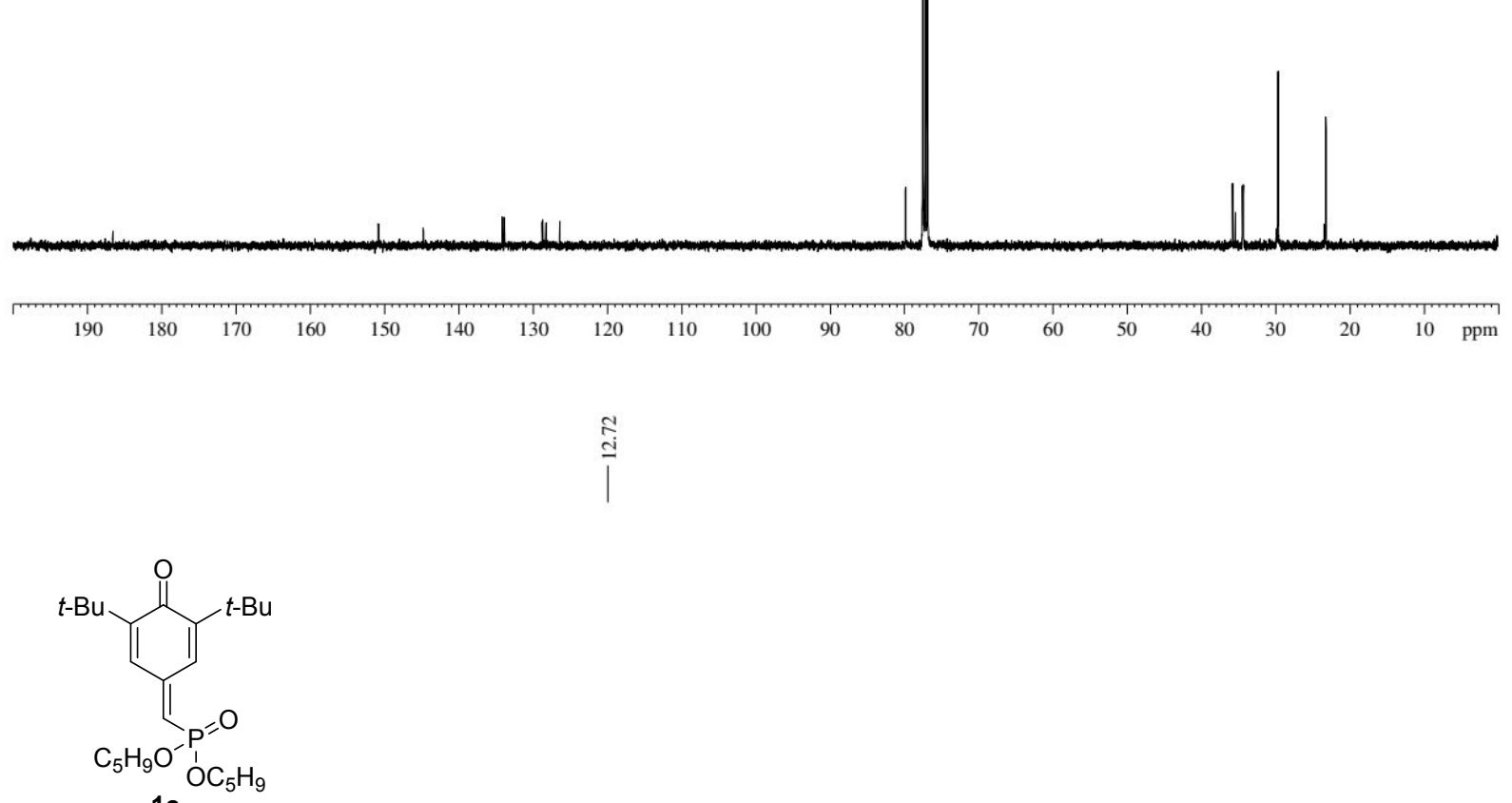

${ }^{31} \mathrm{P}-\mathrm{NMR}$ (162 MHz, CDCl3)

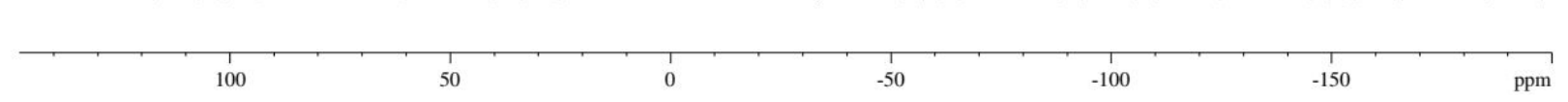



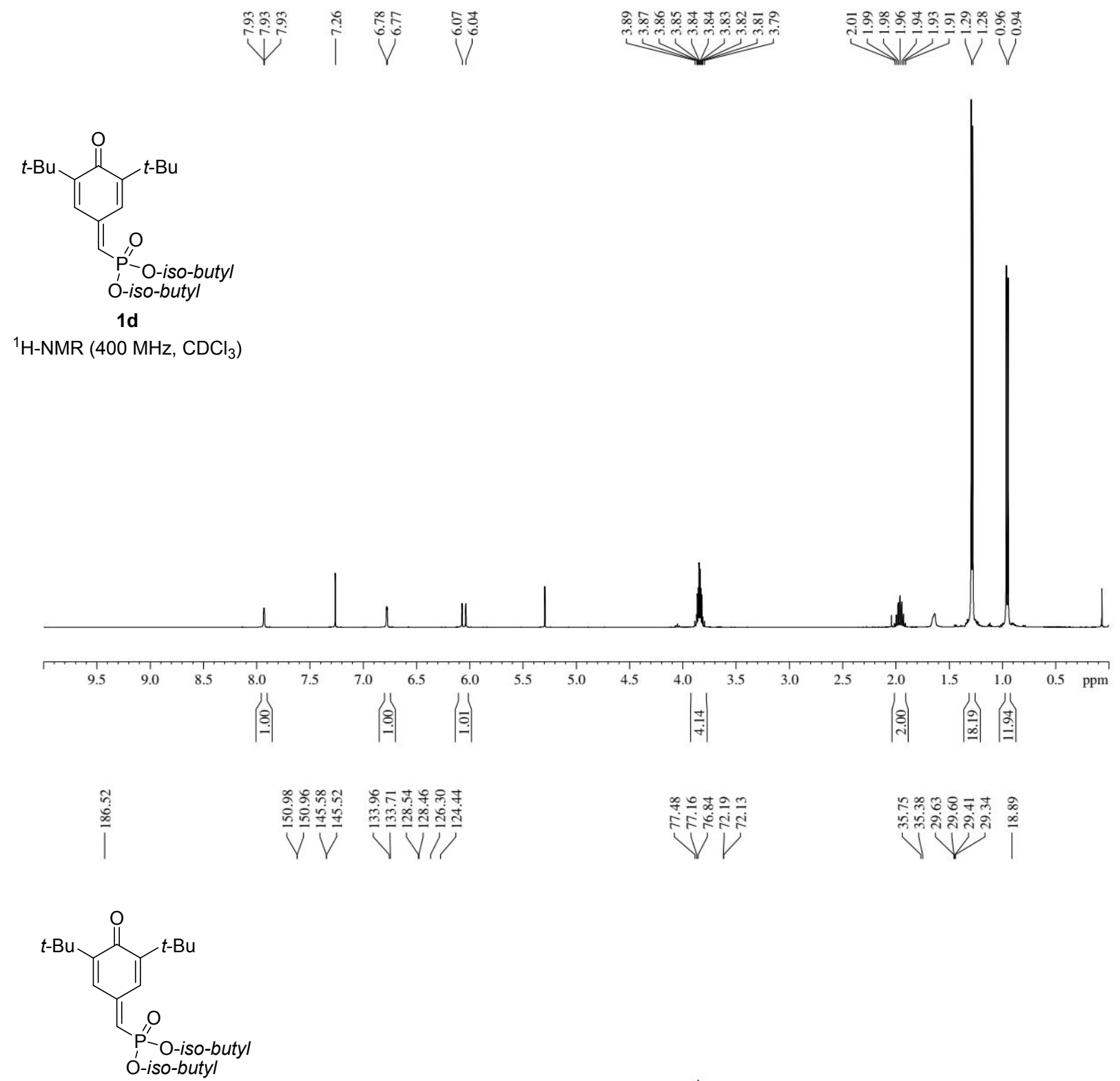

1d

${ }^{13} \mathrm{C}\left\{{ }^{1} \mathrm{H}\right\}-\mathrm{NMR}\left(100 \mathrm{MHz}, \mathrm{CDCl}_{3}\right)$
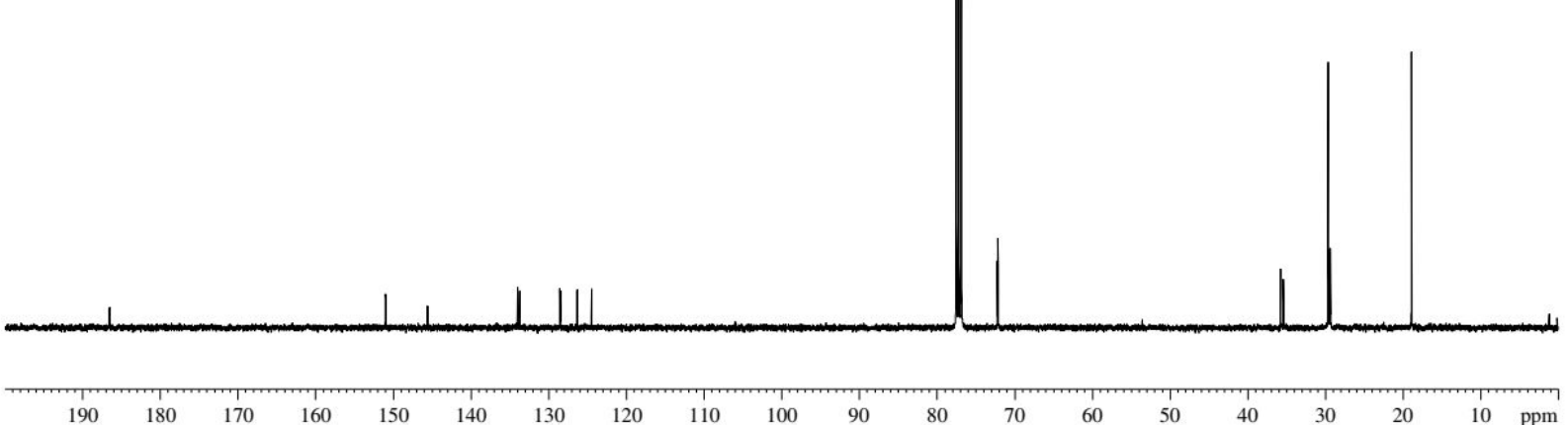


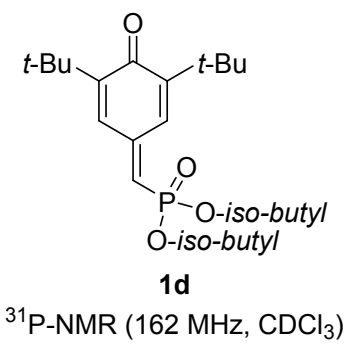

${ }^{31}$ P-NMR $\left(162 \mathrm{MHz}, \mathrm{CDCl}_{3}\right)$
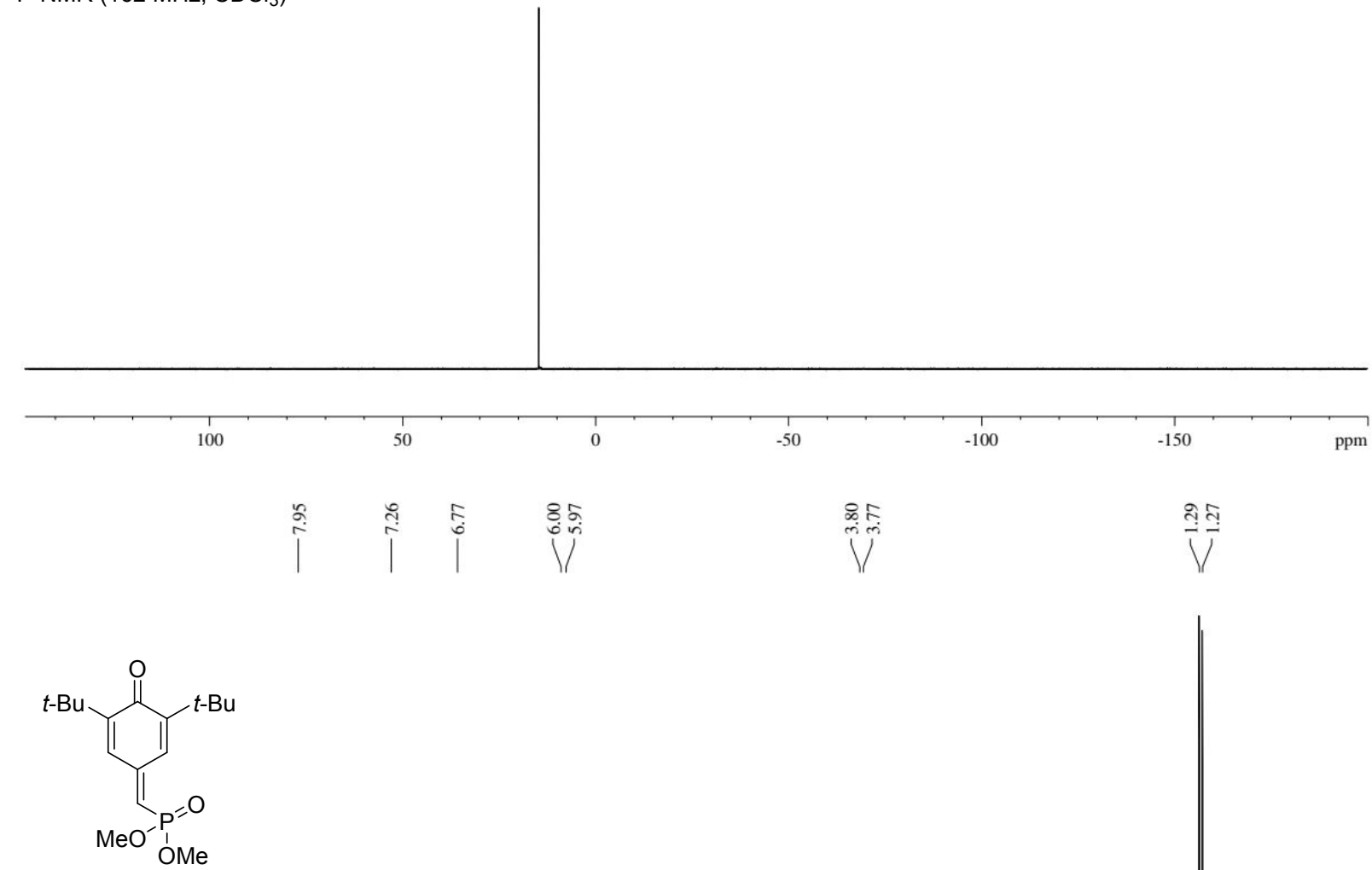

$1 \mathrm{e}$

${ }^{1} \mathrm{H}-\mathrm{NMR}\left(400 \mathrm{MHz}, \mathrm{CDCl}_{3}\right)$

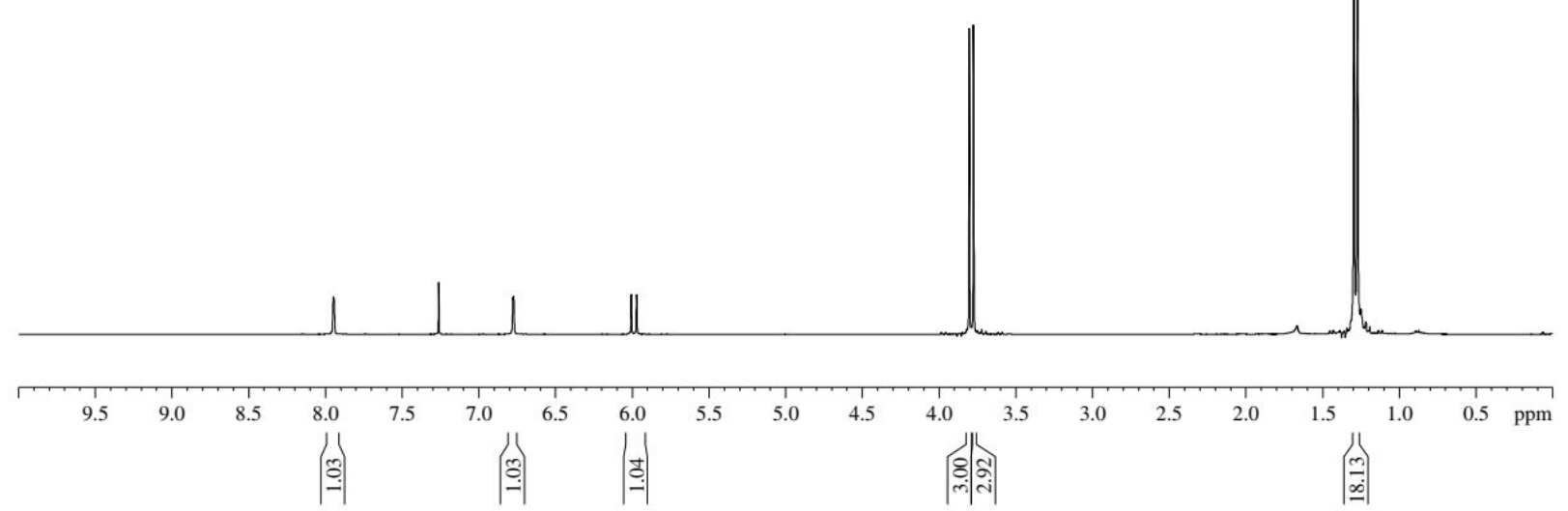




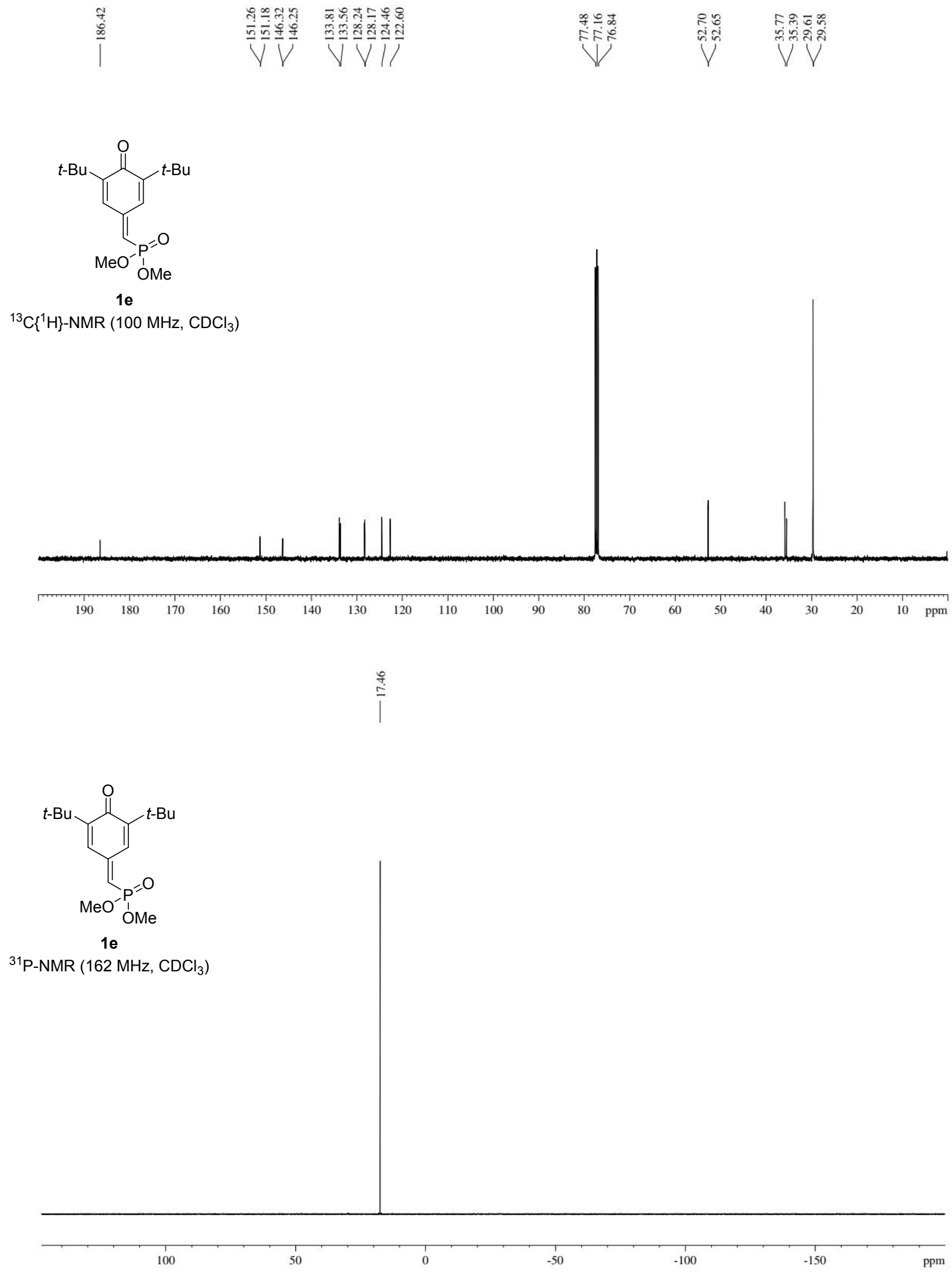



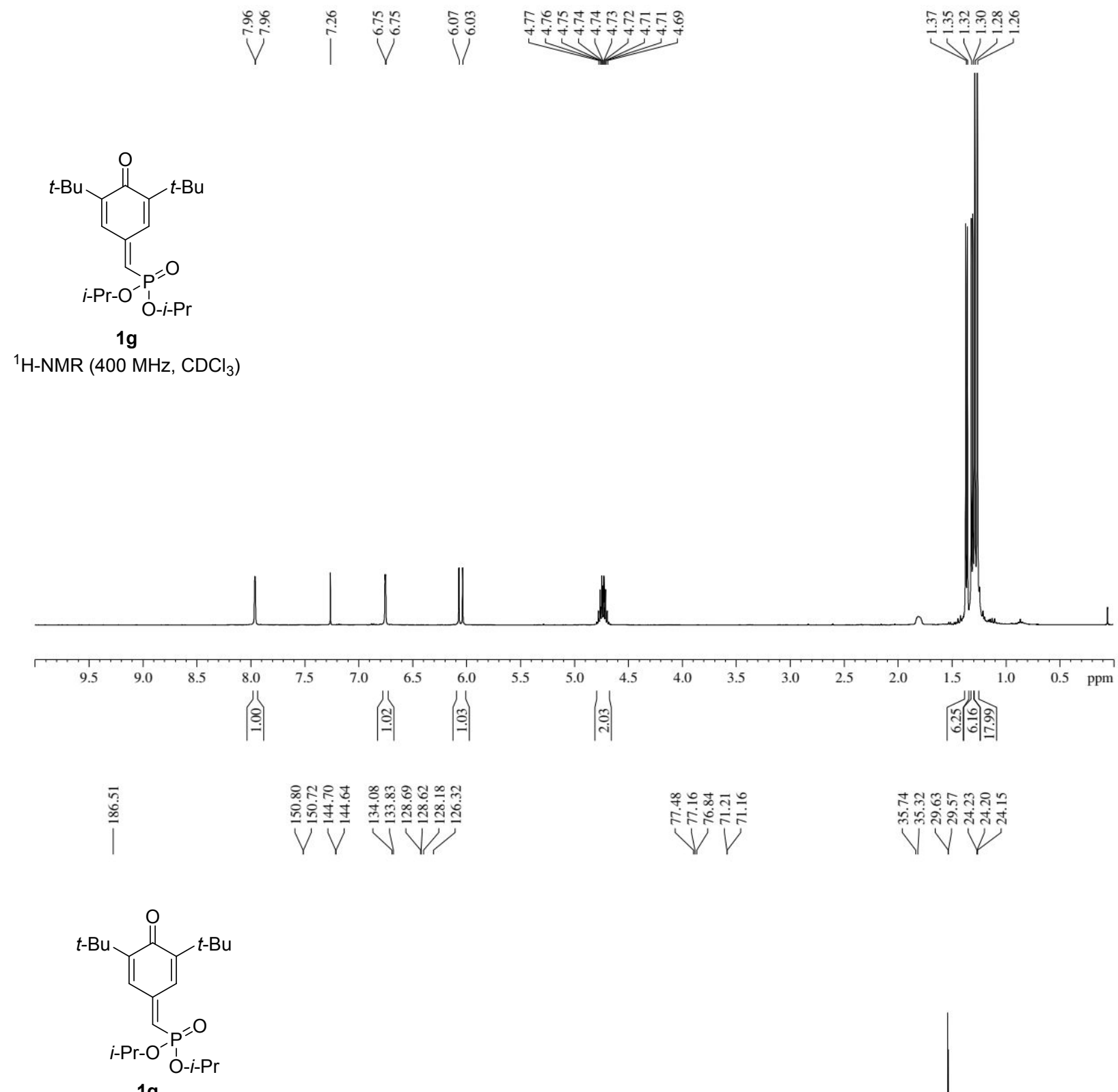

${ }^{13} \mathrm{C}\left\{{ }^{1} \mathrm{H}\right\}-\mathrm{NMR}\left(100 \mathrm{MHz}, \mathrm{CDCl}_{3}\right)$

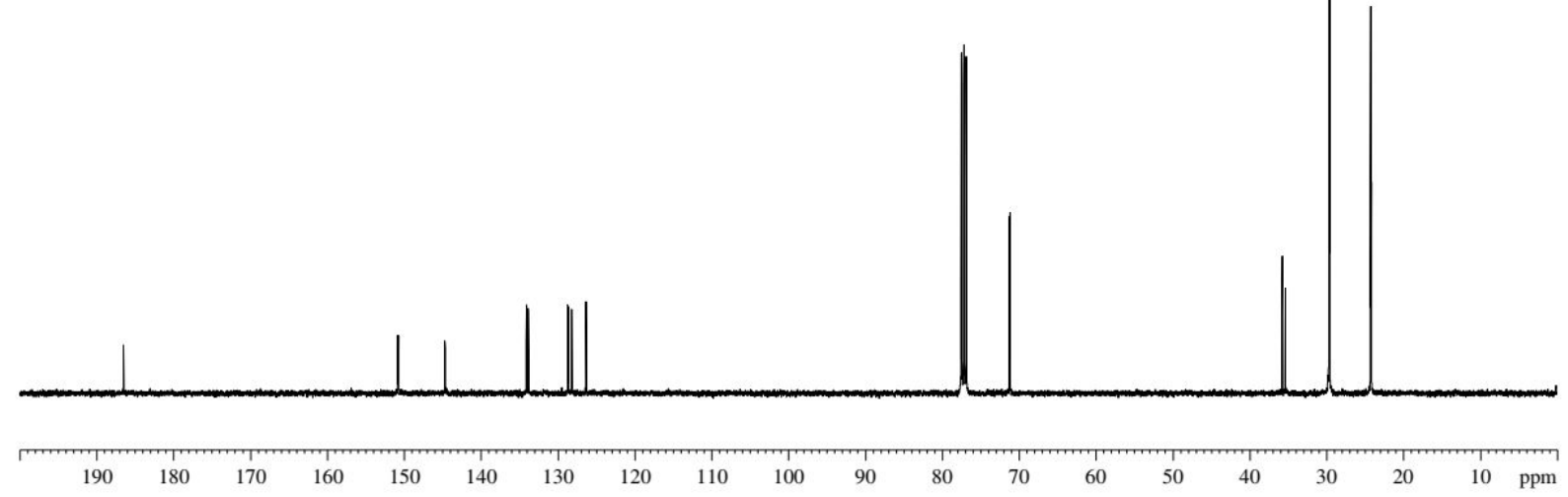




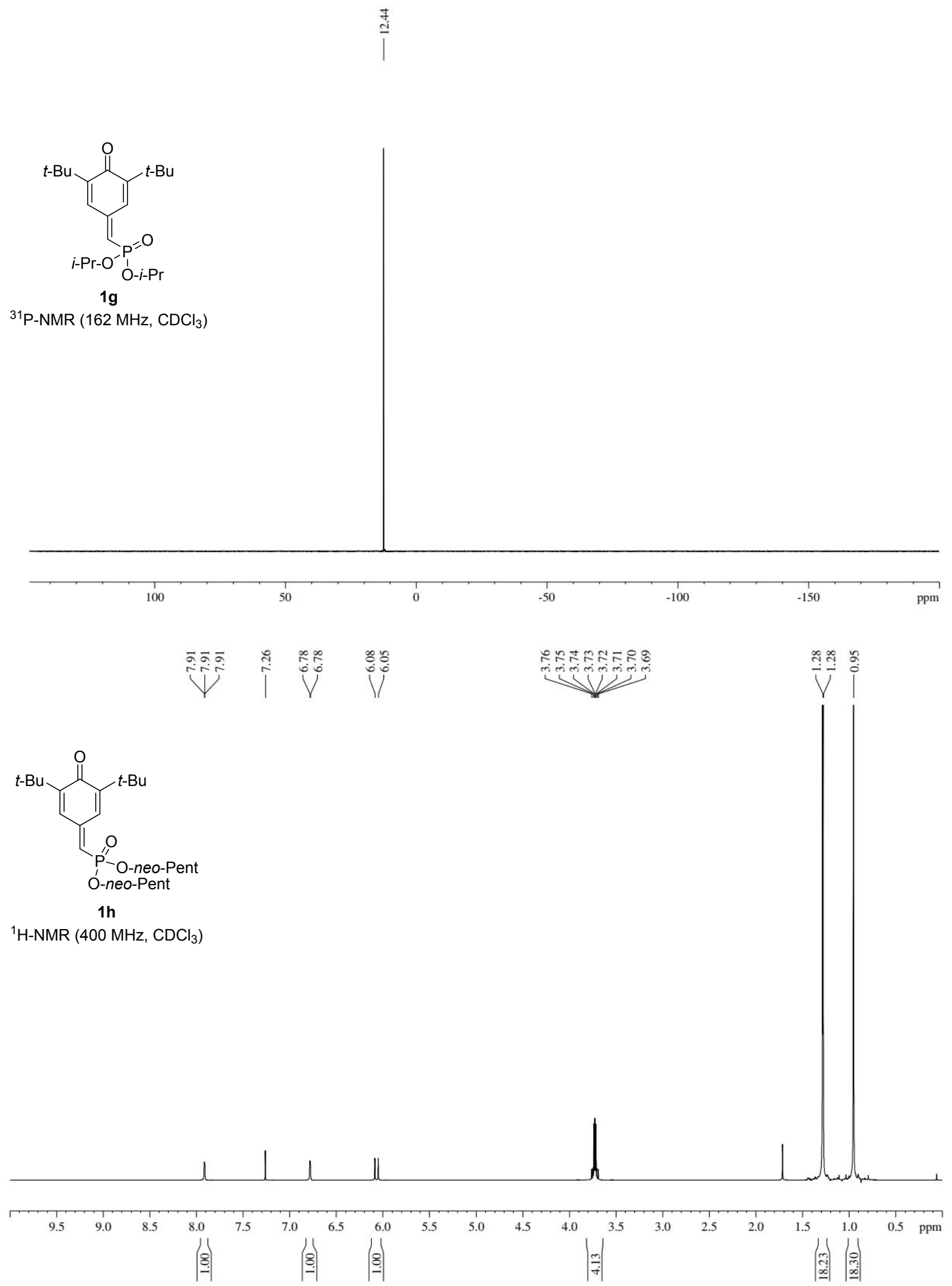



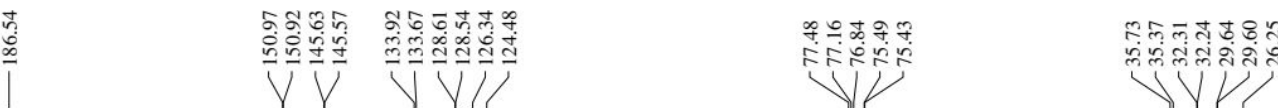

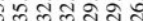

,

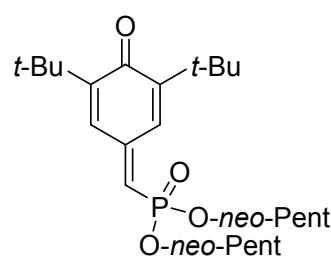

$1 \mathrm{~h}$

${ }^{13} \mathrm{C}\left\{{ }^{1} \mathrm{H}\right\}-\mathrm{NMR}\left(100 \mathrm{MHz}, \mathrm{CDCl}_{3}\right)$

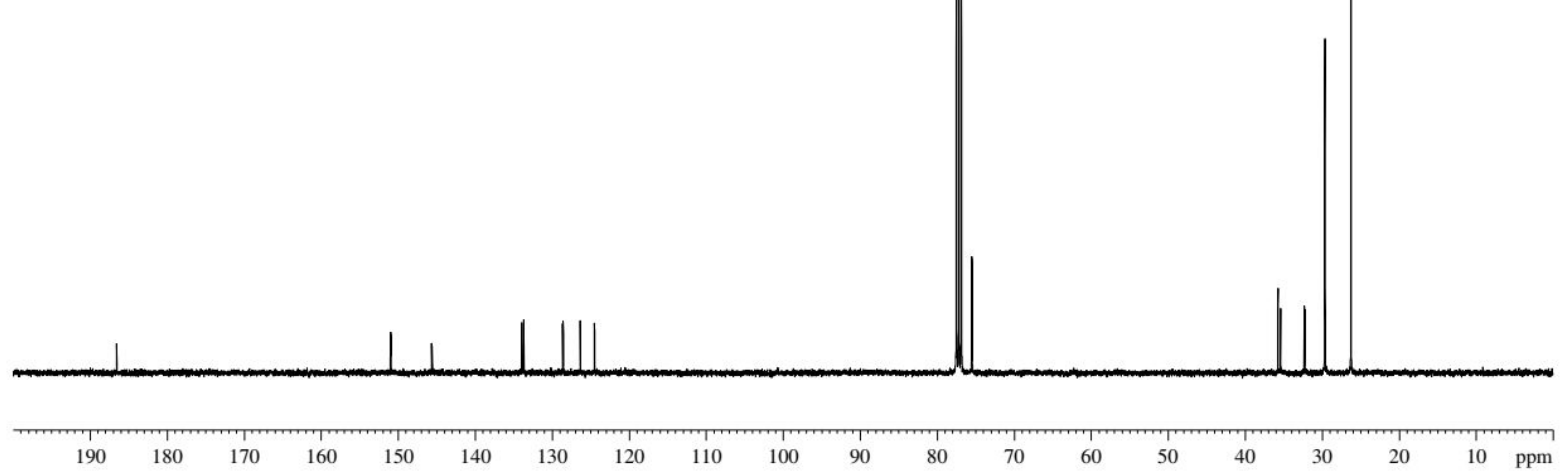

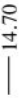

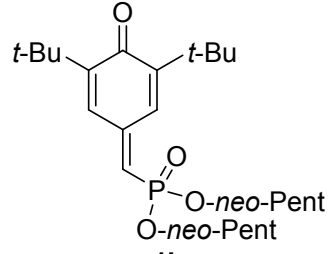

$1 \mathrm{~h}$

${ }^{31} \mathrm{P}-\mathrm{NMR}\left(162 \mathrm{MHz}, \mathrm{CDCl}_{3}\right)$

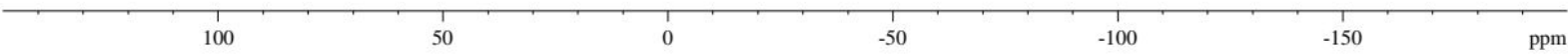



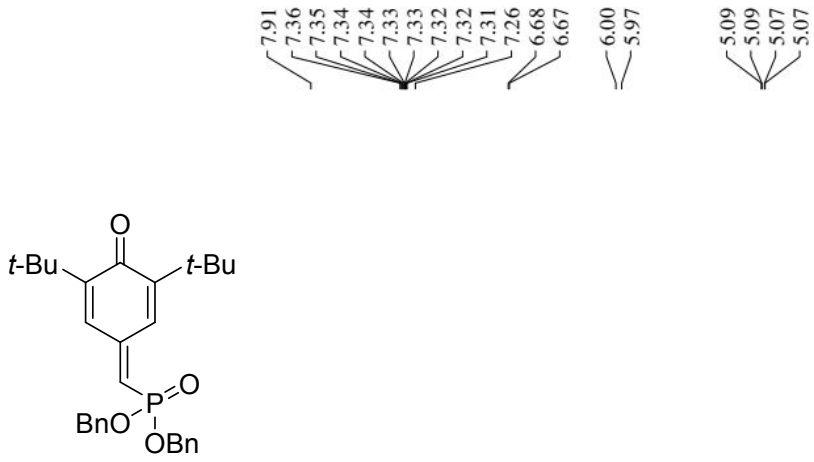

$1 \mathrm{i}$

${ }^{1} \mathrm{H}-\mathrm{NMR}\left(400 \mathrm{MHz}, \mathrm{CDCl}_{3}\right)$
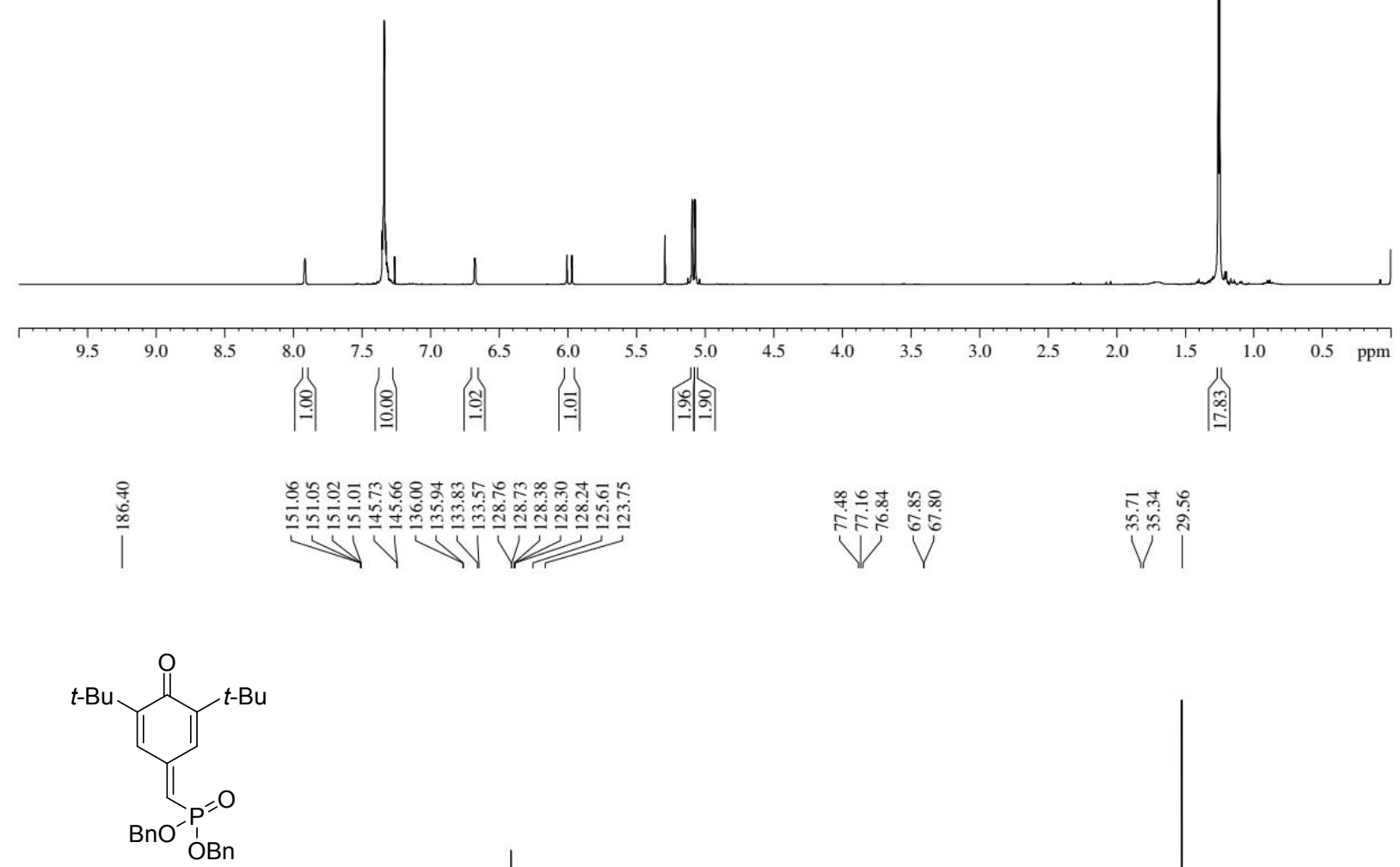

$1 \mathrm{i}$

${ }^{13} \mathrm{C}\left\{{ }^{1} \mathrm{H}\right\}$-NMR $\left(100 \mathrm{MHz}, \mathrm{CDCl}_{3}\right)$

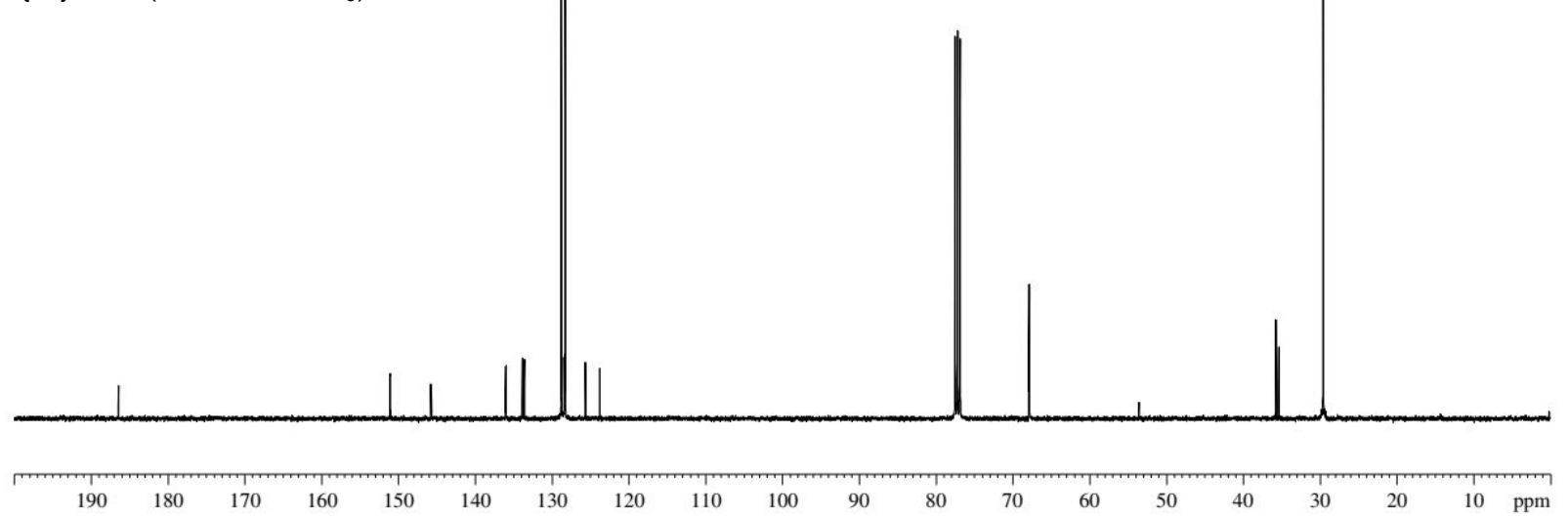




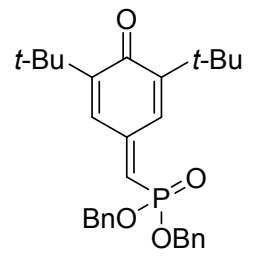

$1 \mathrm{i}$

${ }^{31} \mathrm{P}-\mathrm{NMR}\left(162 \mathrm{MHz}, \mathrm{CDCl}_{3}\right)$

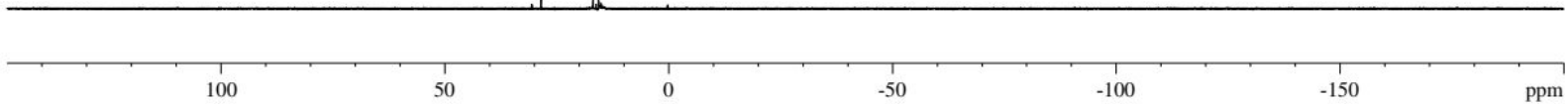

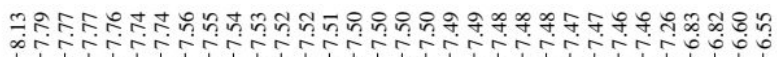

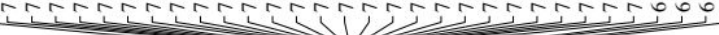

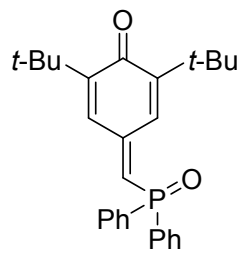

$1 \mathrm{j}$

${ }^{1} \mathrm{H}-\mathrm{NMR}\left(400 \mathrm{MHz}, \mathrm{CDCl}_{3}\right)$

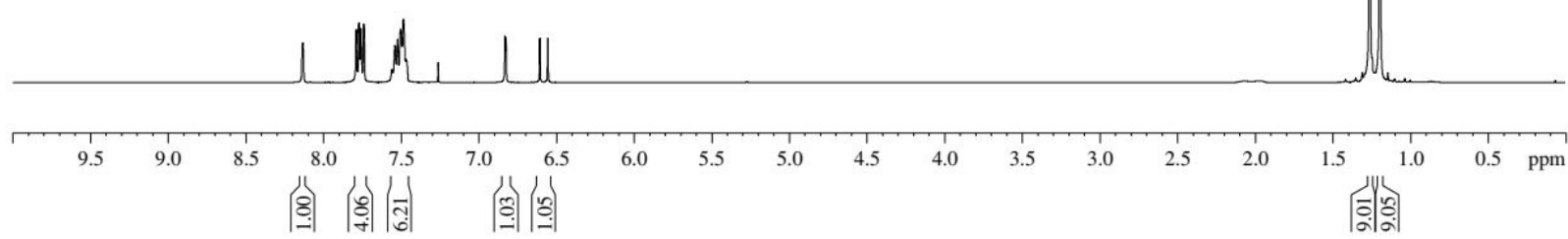



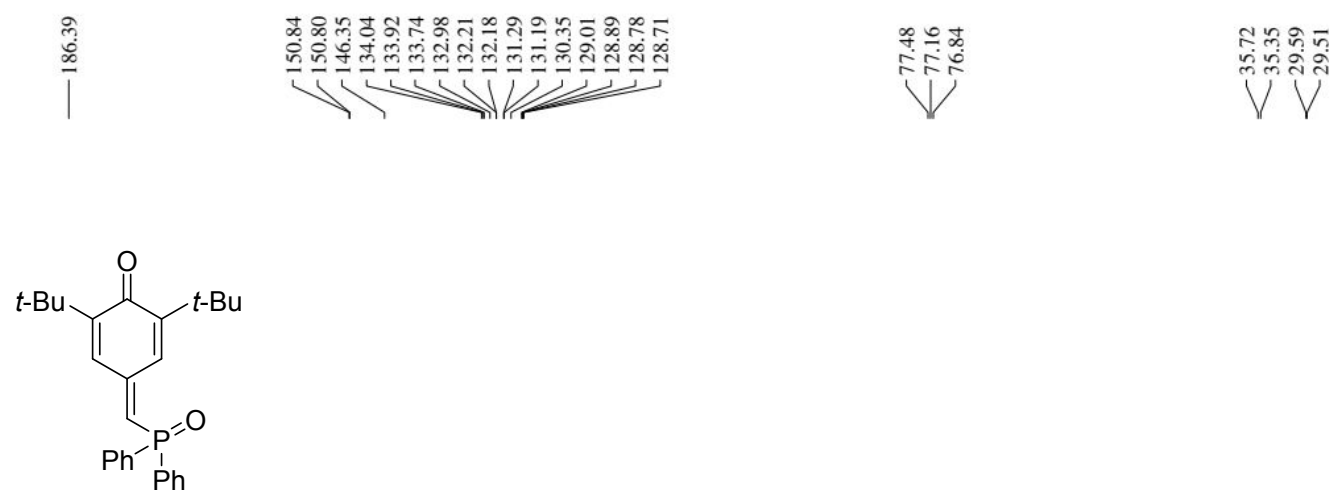

$1 \mathrm{j}$

${ }^{13} \mathrm{C}\left\{{ }^{1} \mathrm{H}\right\}-\mathrm{NMR}\left(100 \mathrm{MHz}, \mathrm{CDCl}_{3}\right)$
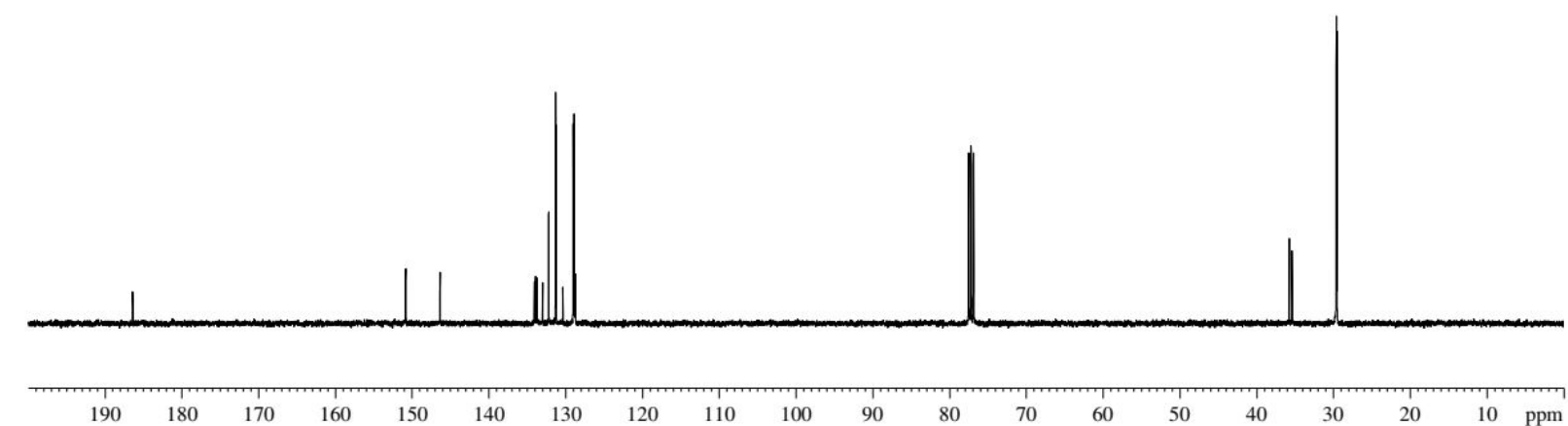

\section{$\frac{5}{i}$}

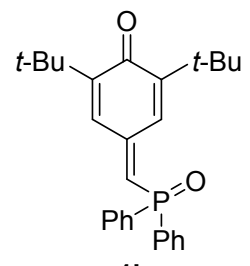

$1 \mathrm{j}$

${ }^{31} \mathrm{P}-\mathrm{NMR}\left(162 \mathrm{MHz}, \mathrm{CDCl}_{3}\right.$ )

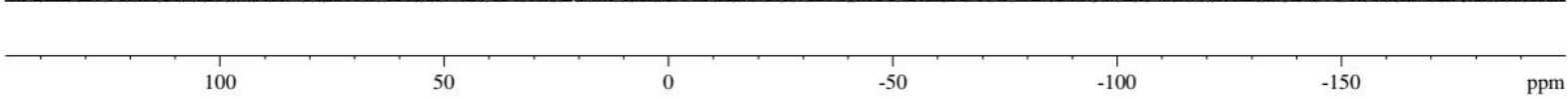




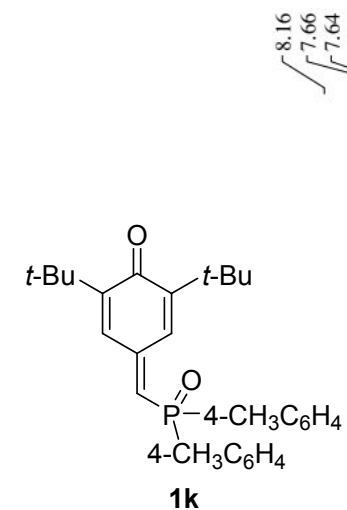

${ }^{1} \mathrm{H}-\mathrm{NMR}\left(400 \mathrm{MHz}, \mathrm{CDCl}_{3}\right)$
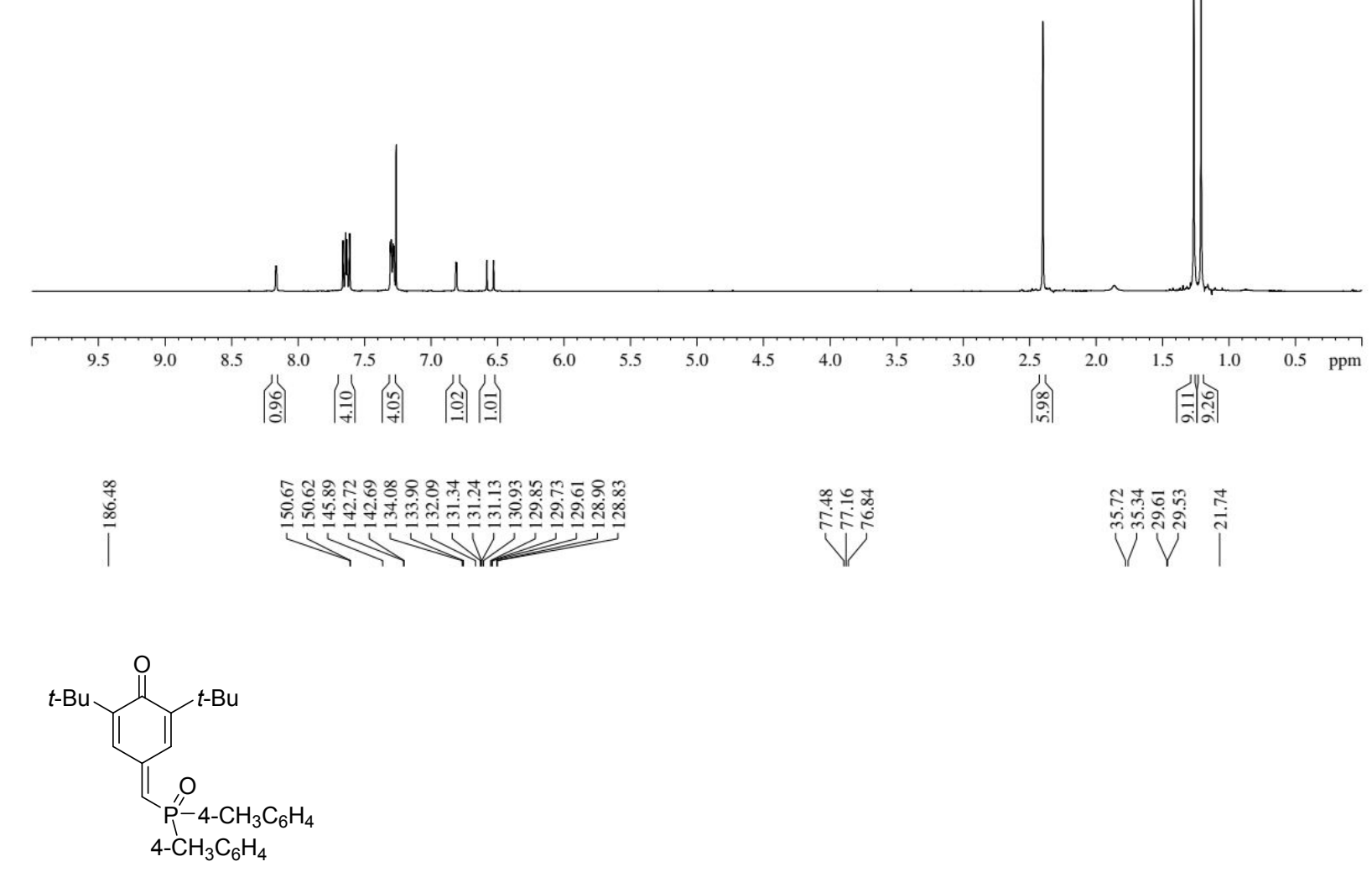

${ }^{13} \mathrm{C}\left\{{ }^{1} \mathrm{H}\right\}-\mathrm{NMR}\left(100 \mathrm{MHz}, \mathrm{CDCl}_{3}\right)$
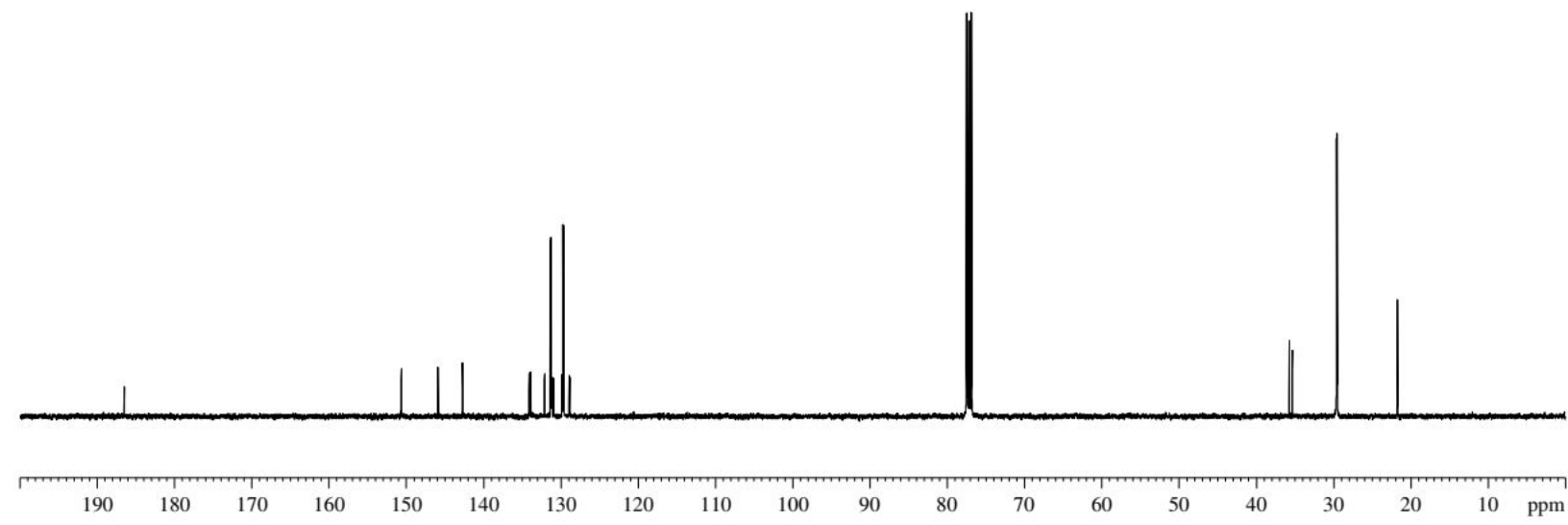


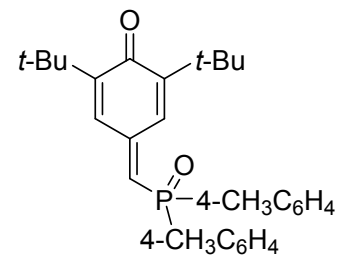

$1 \mathrm{k}$

${ }^{31} \mathrm{P}-\mathrm{NMR}\left(162 \mathrm{MHz}, \mathrm{CDCl}_{3}\right.$ )
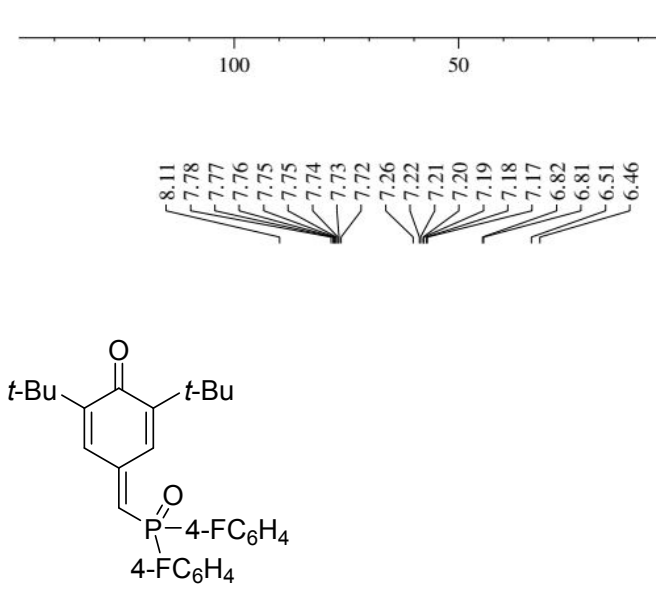

11

${ }^{1} \mathrm{H}-\mathrm{NMR}\left(400 \mathrm{MHz}, \mathrm{CDCl}_{3}\right.$ )

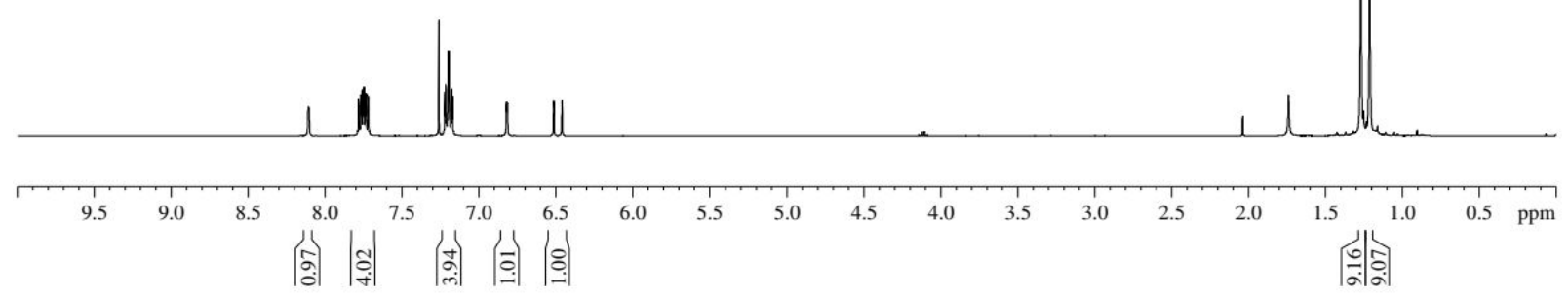




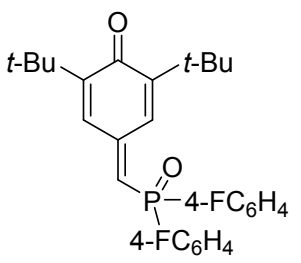

11

${ }^{13} \mathrm{C}\left\{{ }^{1} \mathrm{H}\right\}-\mathrm{NMR}\left(100 \mathrm{MHz}, \mathrm{CDCl}_{3}\right)$

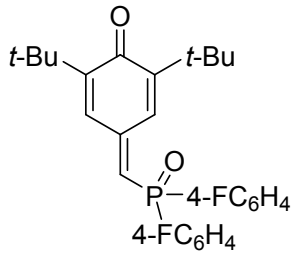

11

${ }^{19} \mathrm{~F}-\mathrm{NMR}\left(376 \mathrm{MHz}, \mathrm{CDCl}_{3}\right.$ )

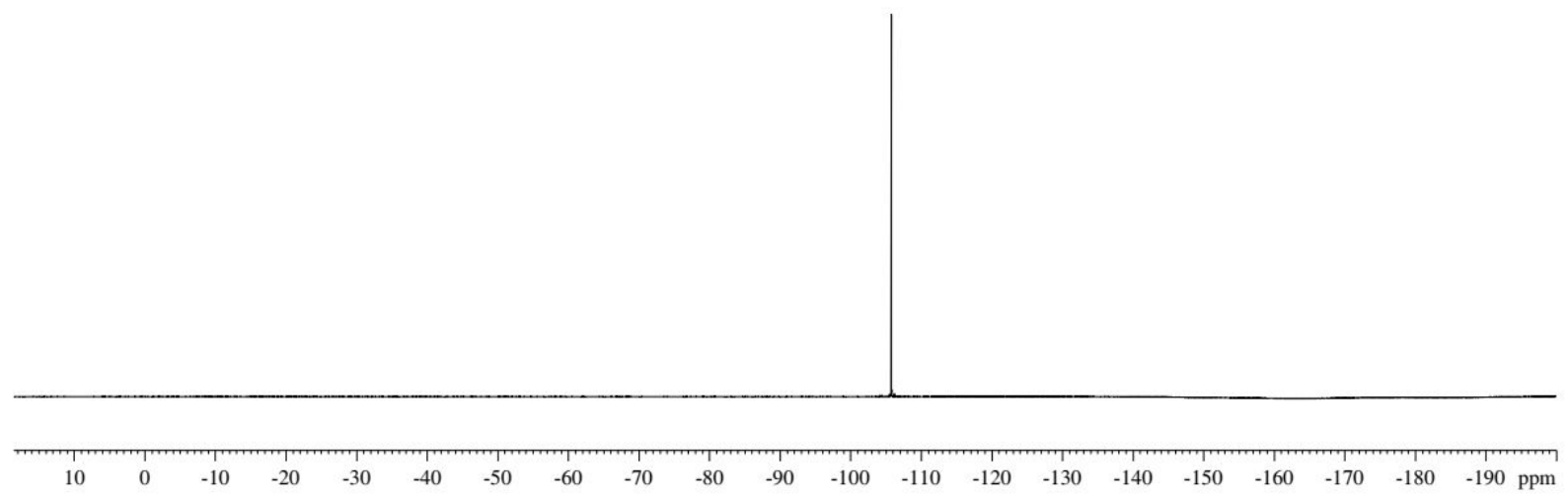




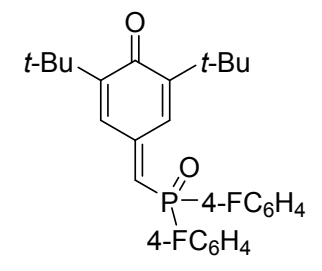

11

${ }^{31} \mathrm{P}-\mathrm{NMR}\left(162 \mathrm{MHz}, \mathrm{CDCl}_{3}\right.$ )
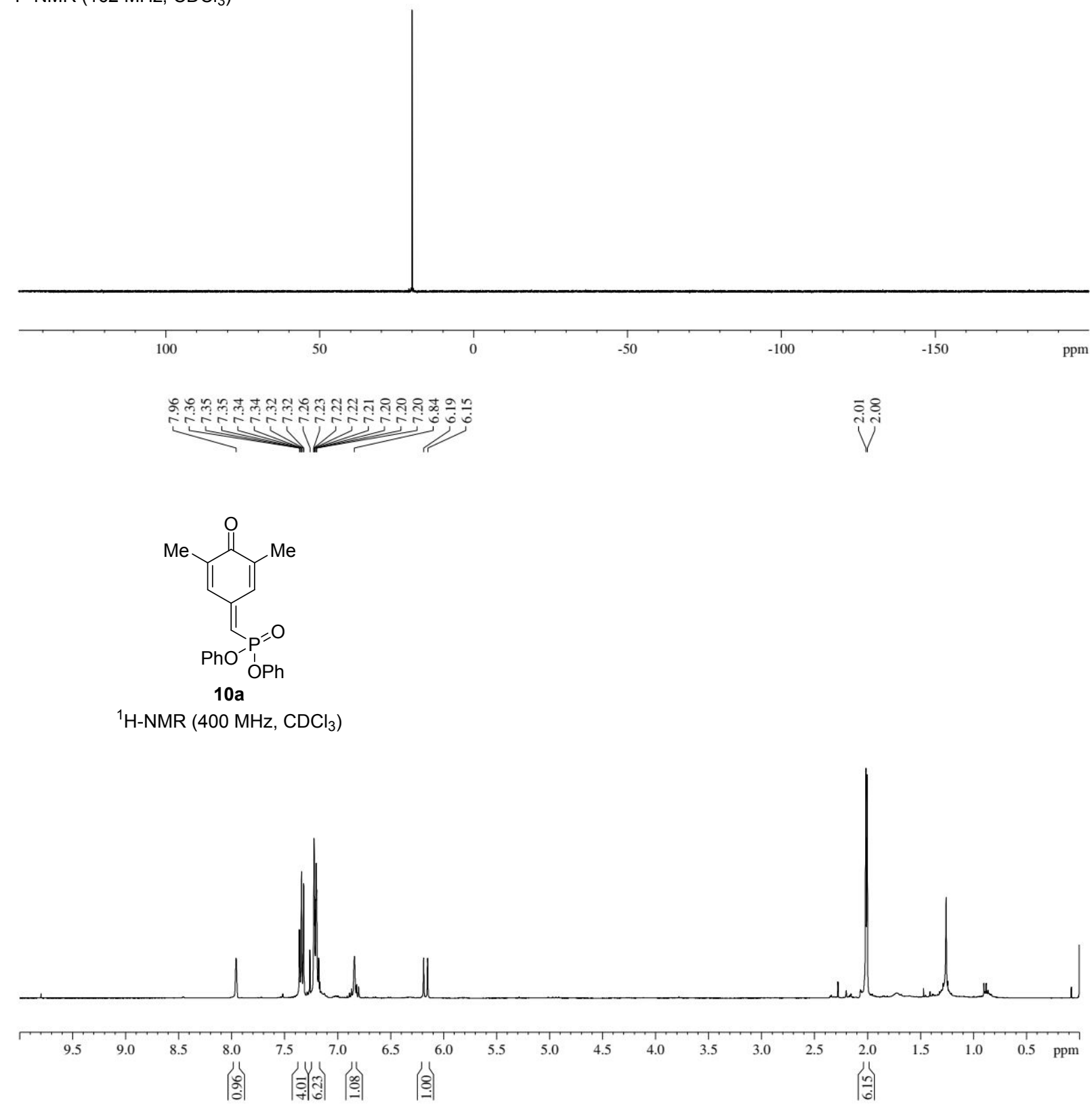

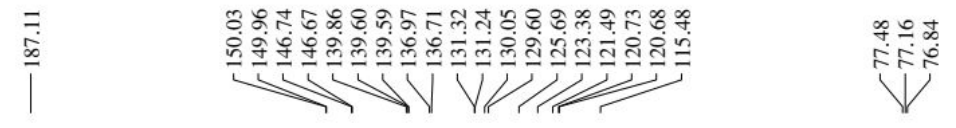

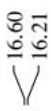

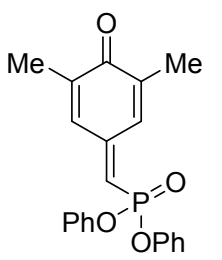

$10 \mathrm{a}$

${ }^{13} \mathrm{C}\left\{{ }^{1} \mathrm{H}\right\}-N M R\left(100 \mathrm{MHz}, \mathrm{CDCl}_{3}\right)$
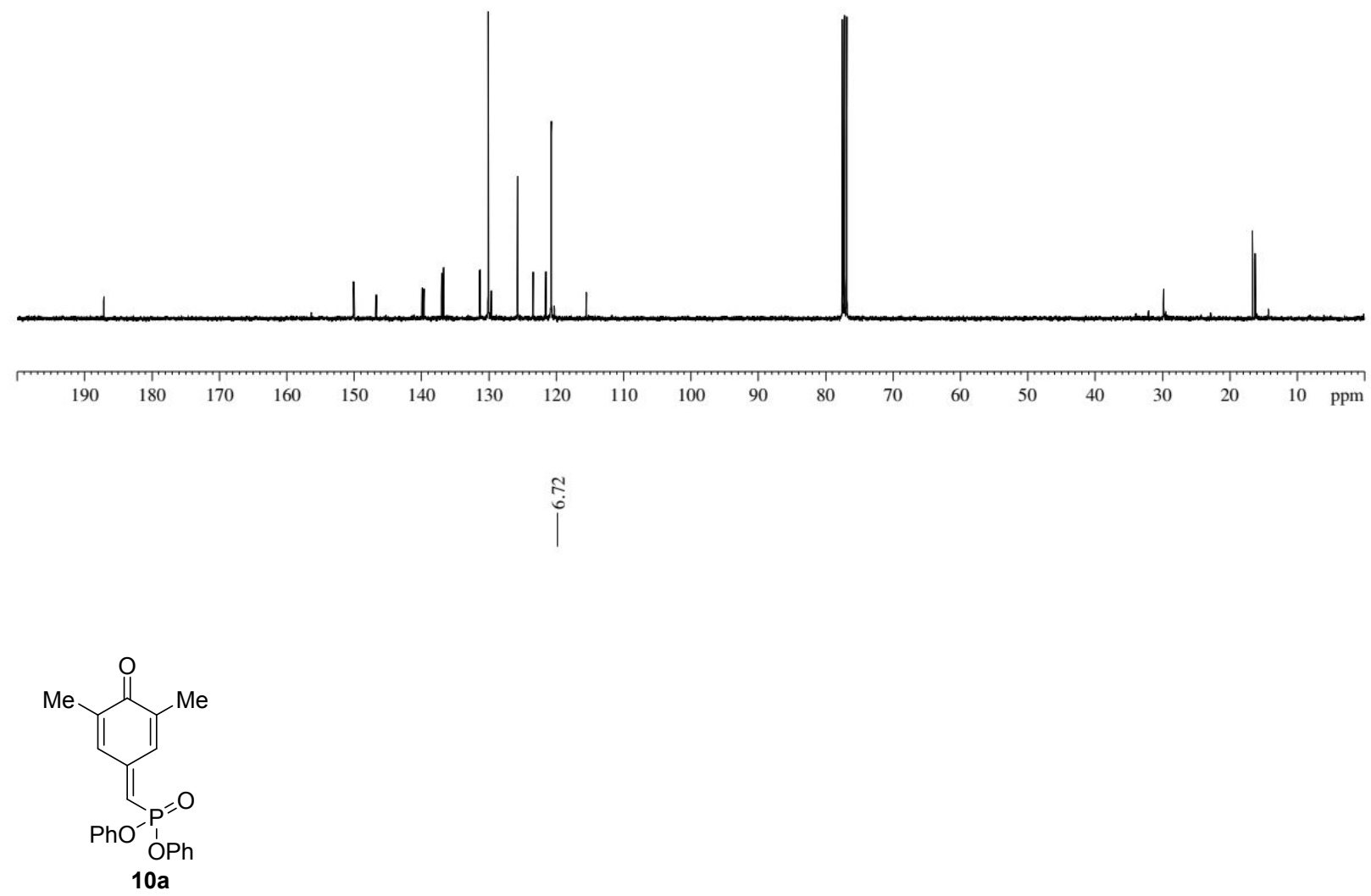

${ }^{31} \mathrm{P}-\mathrm{NMR}\left(162 \mathrm{MHz}, \mathrm{CDCl}_{3}\right.$ )

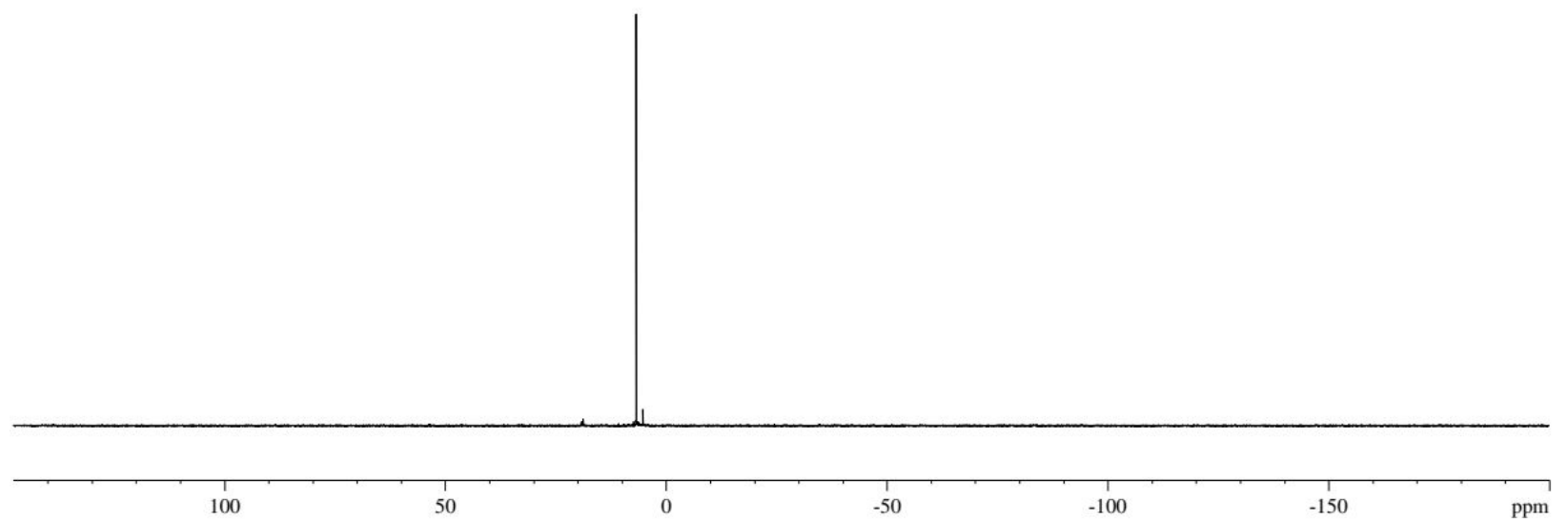



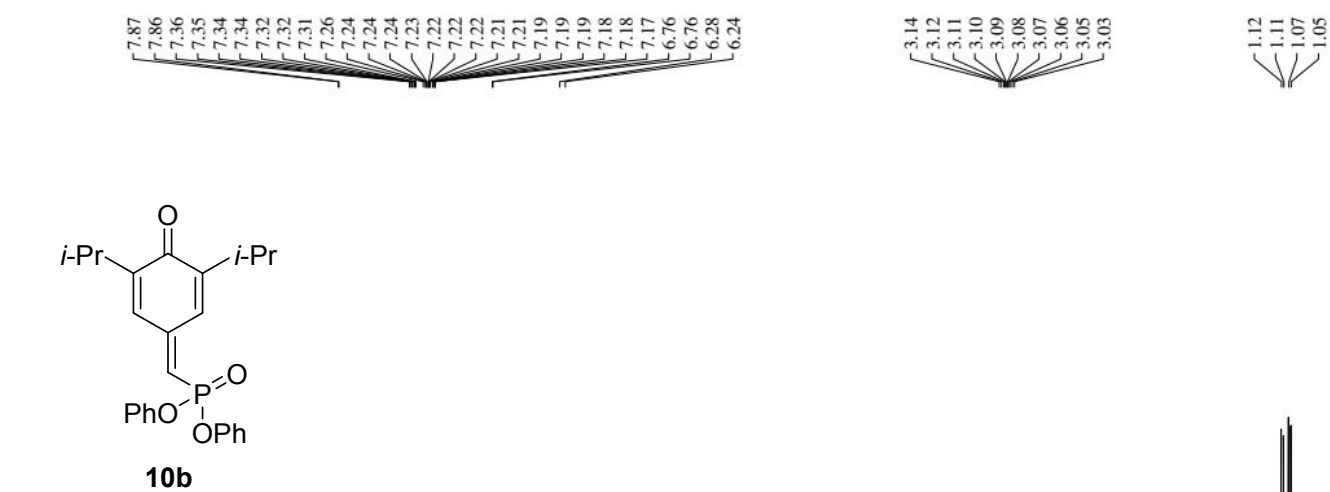

${ }^{1} \mathrm{H}-\mathrm{NMR}\left(400 \mathrm{MHz}, \mathrm{CDCl}_{3}\right)$
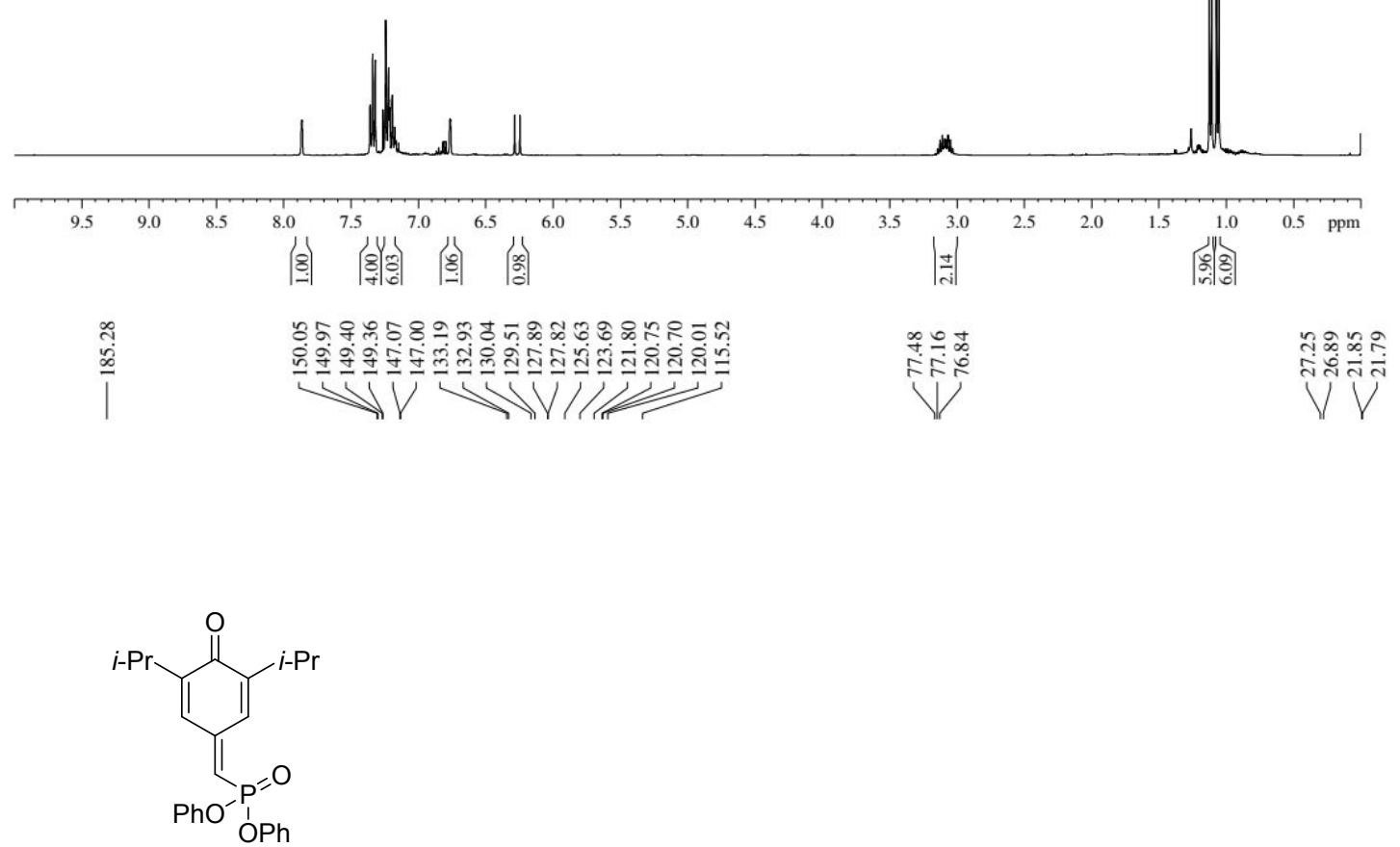

$10 \mathrm{~b}$

${ }^{13} \mathrm{C}\left\{{ }^{1} \mathrm{H}\right\}-\mathrm{NMR}\left(100 \mathrm{MHz}, \mathrm{CDCl}_{3}\right)$
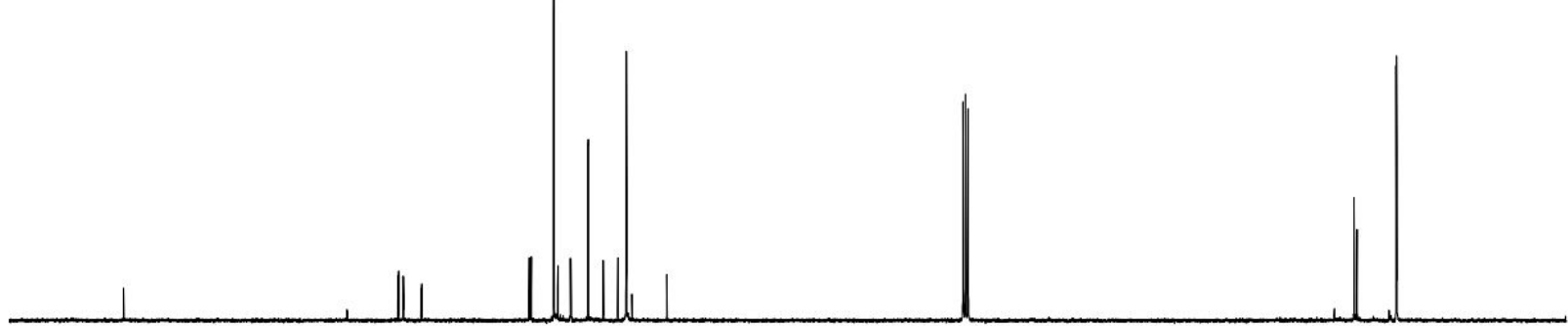

$\begin{array}{lllllllllllllllllll}190 & 180 & 170 & 160 & 150 & 140 & 130 & 120 & 110 & 100 & 90 & 80 & 70 & 60 & 50 & 40 & 30 & 20 & 10\end{array}$ 


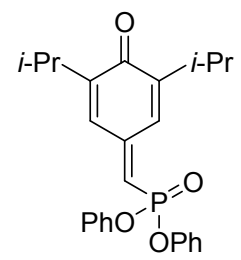

$10 \mathrm{~b}$

${ }^{31} \mathrm{P}-\mathrm{NMR}\left(162 \mathrm{MHz}, \mathrm{CDCl}_{3}\right)$

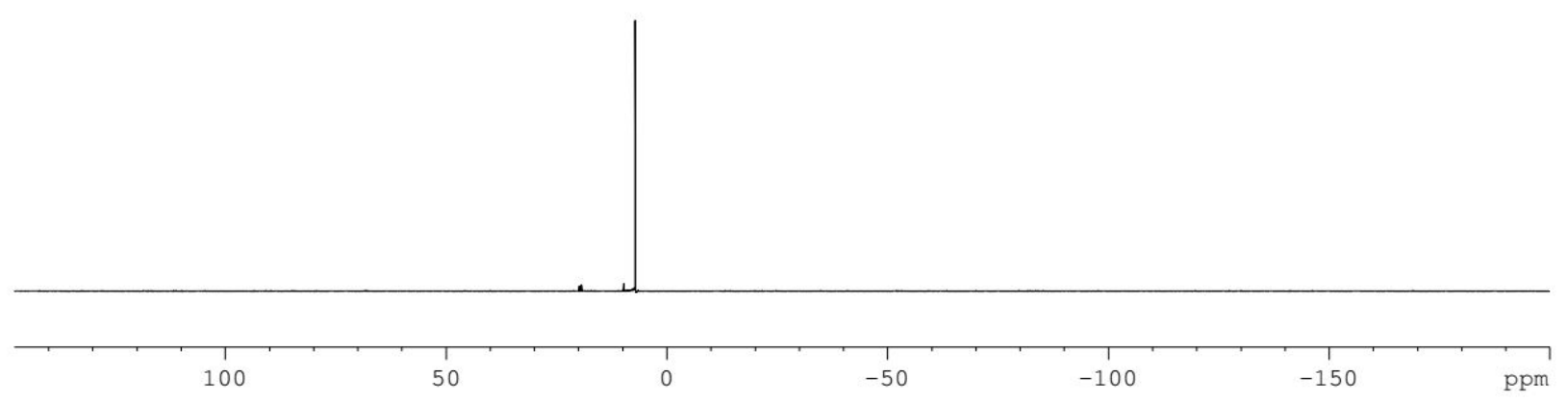

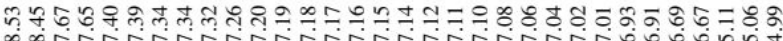

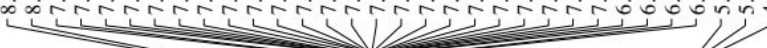

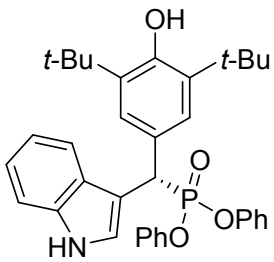

3a

${ }^{1} \mathrm{H}-\mathrm{NMR}\left(400 \mathrm{MHz}, \mathrm{CDCl}_{3}\right)$

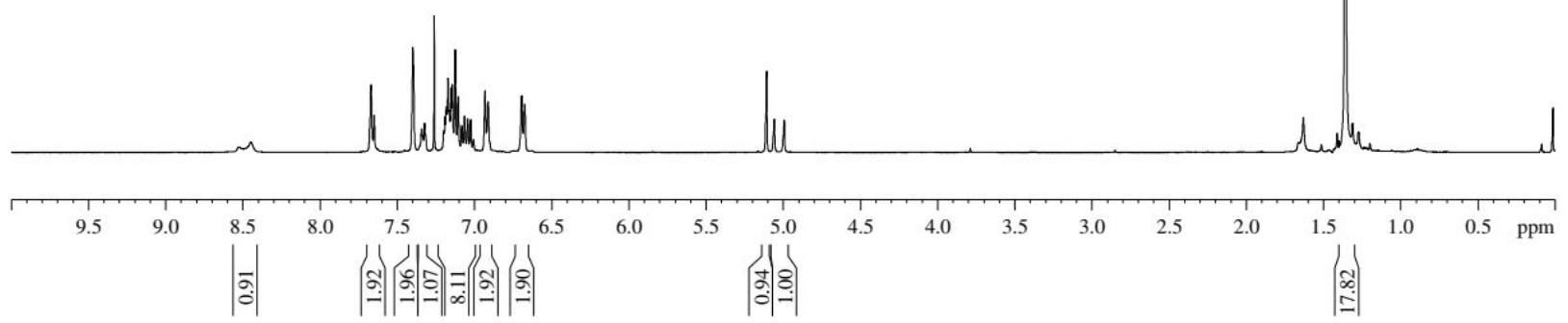




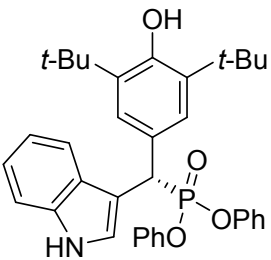

3a

${ }^{13} \mathrm{C}\left\{{ }^{1} \mathrm{H}\right\}-N M R\left(100 \mathrm{MHz}, \mathrm{CDCl}_{3}\right)$
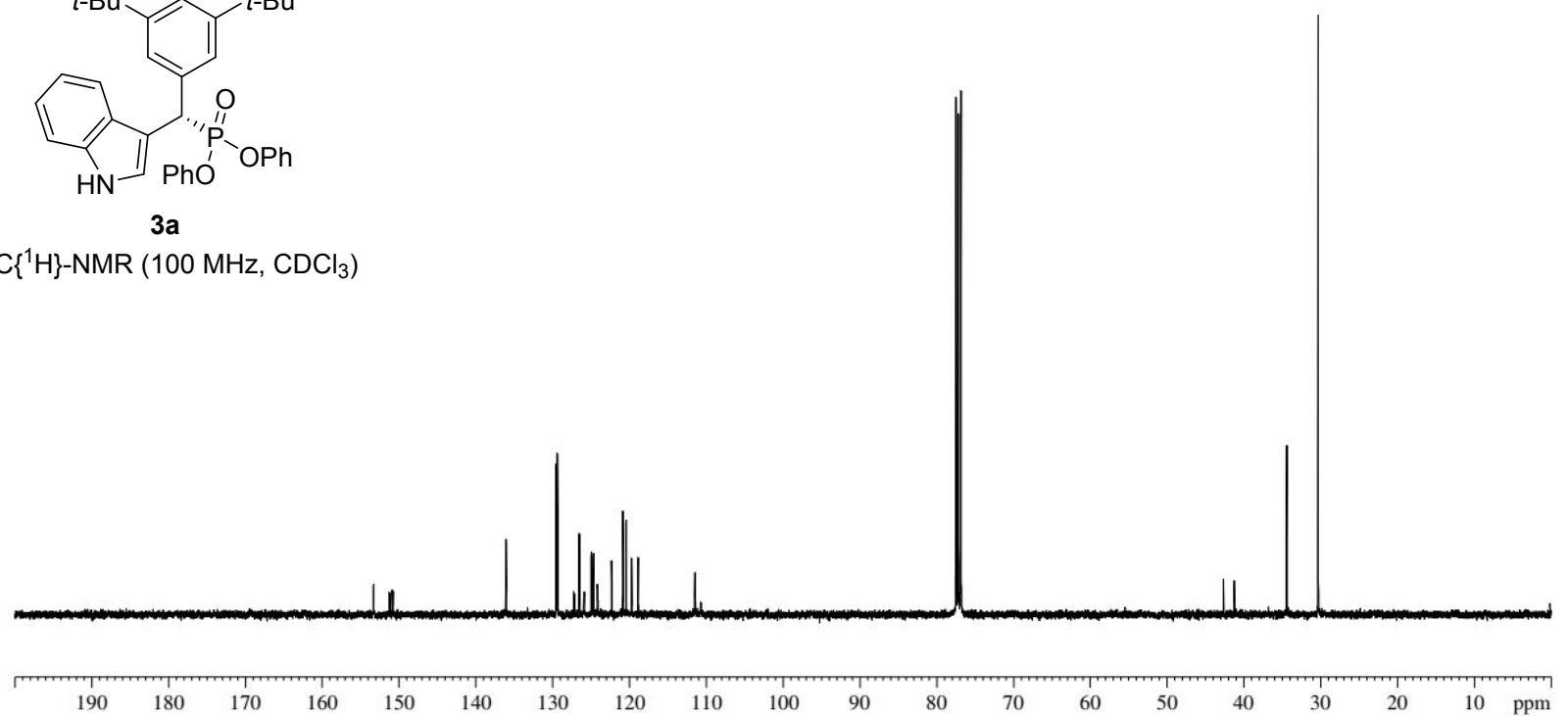

$\stackrel{\widetilde{6}}{1}$

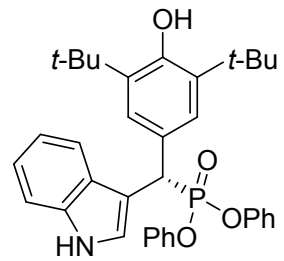

$3 a$

${ }^{31} \mathrm{P}-\mathrm{NMR}\left(162 \mathrm{MHz}, \mathrm{CDCl}_{3}\right.$ )
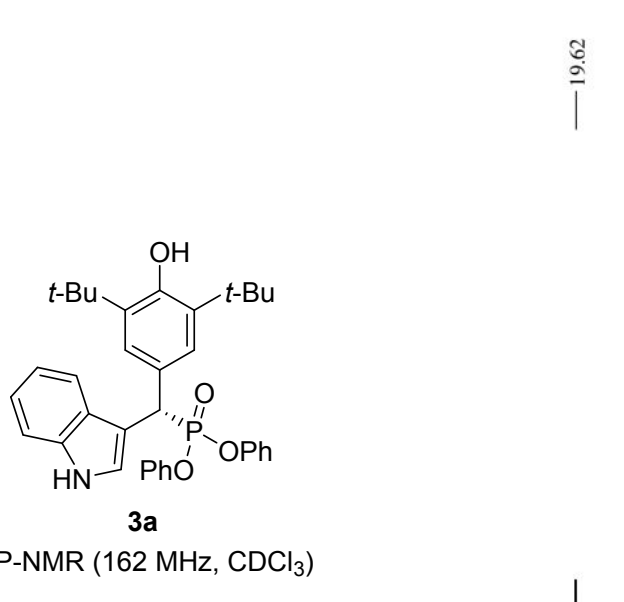
mAU

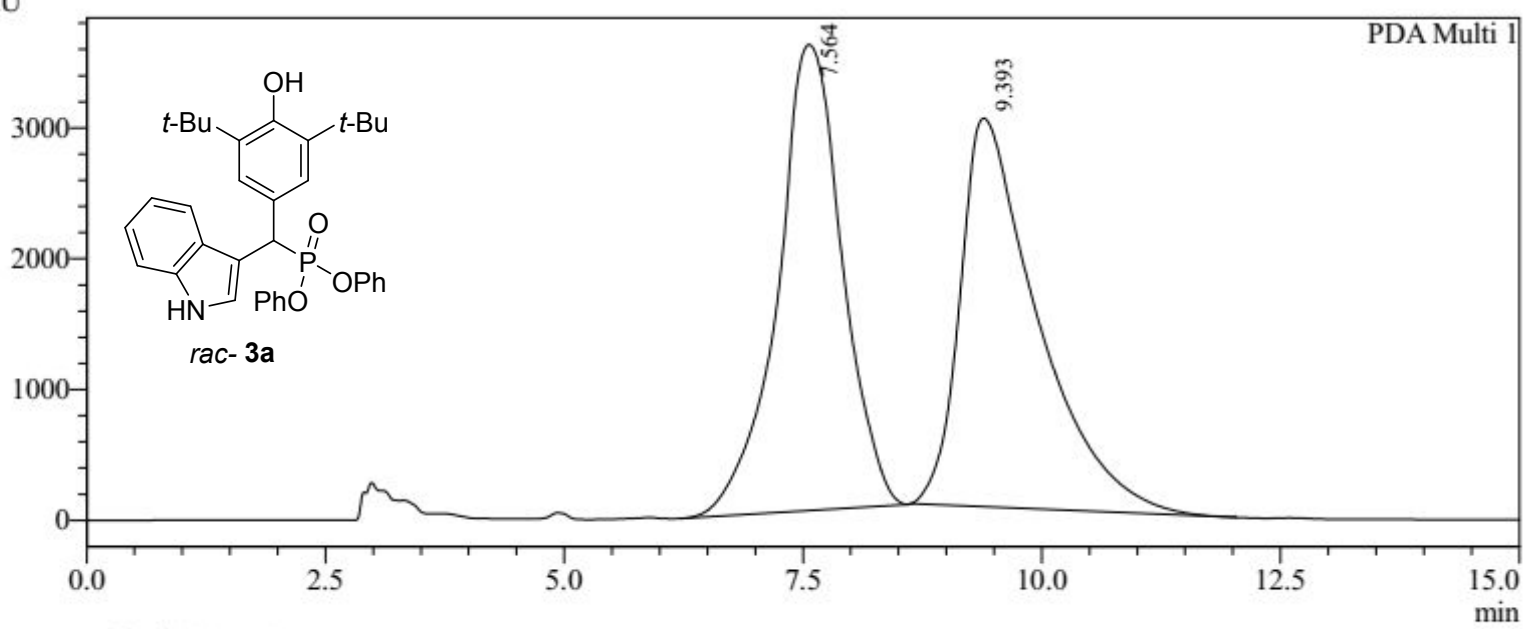

1 PDA Multi $1 / 230 \mathrm{~nm}, 4 \mathrm{~nm}$

\begin{tabular}{|c|c|c|c|c|}
\hline \multicolumn{5}{|c|}{ PDA Chl 230nm } \\
\hline Peak\# & Ret. Time & Area & Height & Area \% \\
\hline 1 & 7.564 & 167798578 & 3562042 & 49.888 \\
\hline 2 & 9.393 & 168554304 & 2968932 & 50.112 \\
\hline Total & & 336352882 & 6530974 & 100.000 \\
\hline
\end{tabular}

mAU

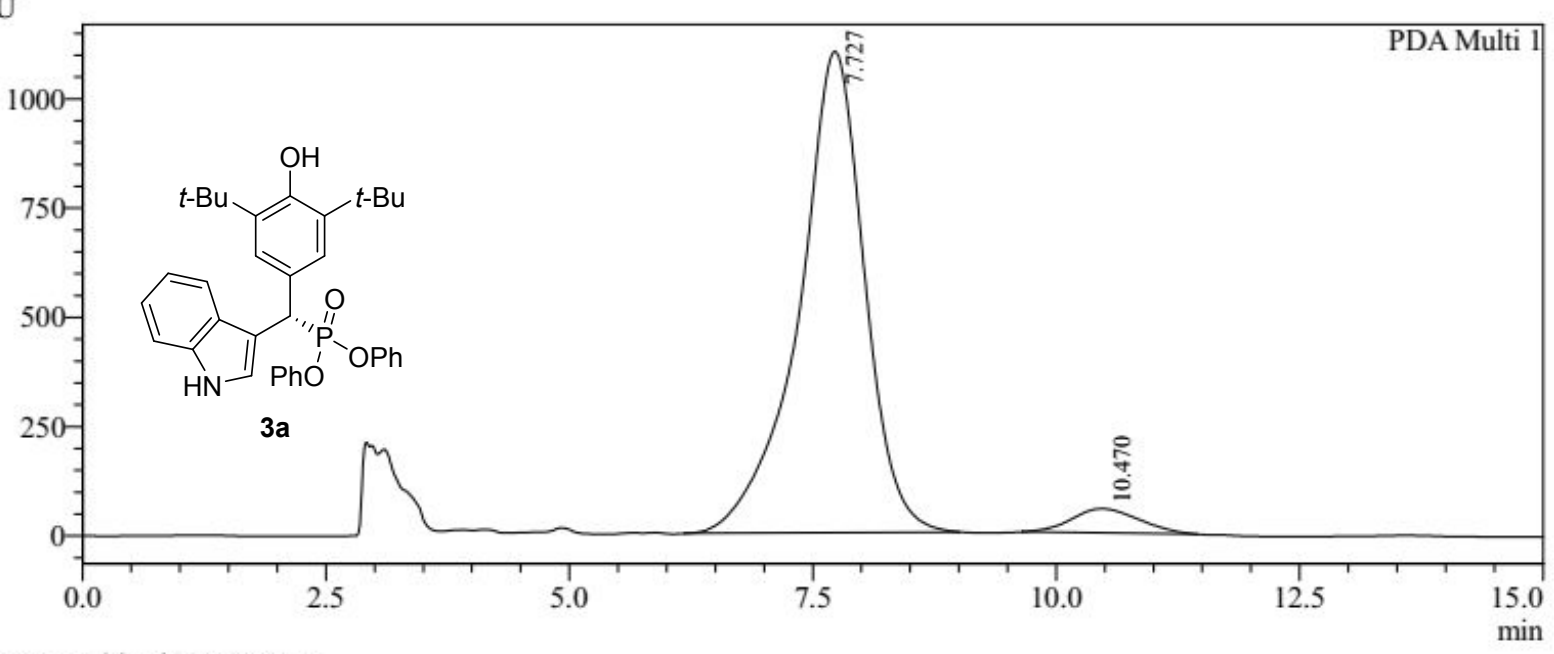

1 PDA Multi $1 / 230 \mathrm{~nm}, 4 \mathrm{~nm}$

\begin{tabular}{|c|c|c|c|c|}
\hline \multicolumn{5}{|c|}{ PDA Chl $230 \mathrm{~nm}$} \\
\hline Peak\# & Ret. Time & Area & Height & Area \% \\
\hline 1 & 7.727 & 51586159 & 1100375 & 95.063 \\
\hline 2 & 10.470 & 2679219 & 55473 & 4.937 \\
\hline Total & & 54265378 & 1155848 & 100.000 \\
\hline
\end{tabular}


<smiles>CCOP(=O)(OCC)C(c1cc(C(C)(C)C)c(O)c(C(C)(C)C)c1)c1c[nH]c2ccccc12</smiles>

3b

${ }^{1} \mathrm{H}-\mathrm{NMR}\left(400 \mathrm{MHz}, \mathrm{CDCl}_{3}\right)$

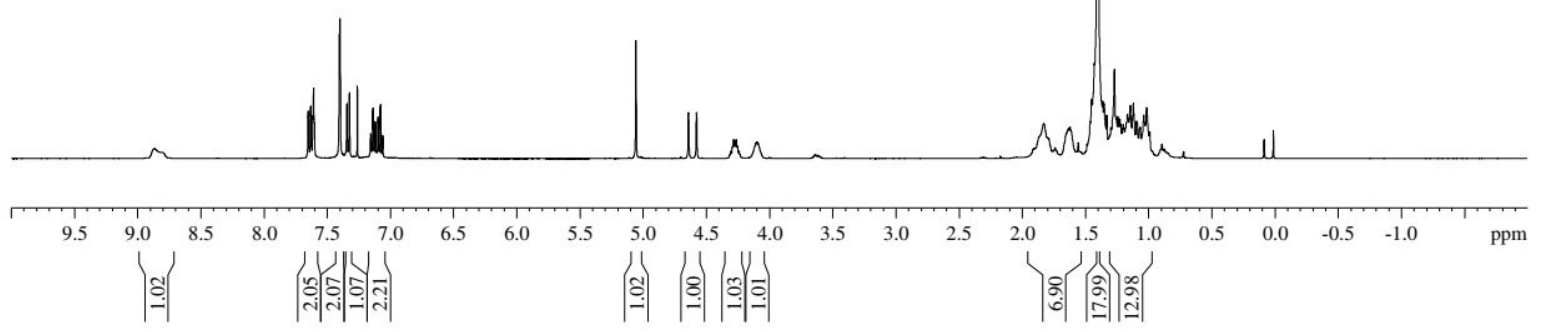

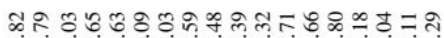
กิ่

$\gamma$

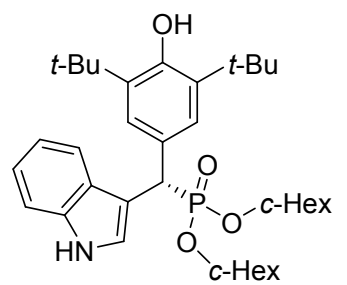

3b

${ }^{13} \mathrm{C}\left\{{ }^{1} \mathrm{H}\right\}$ NMR $\left(100 \mathrm{MHz}, \mathrm{CDCl}_{3}\right)$

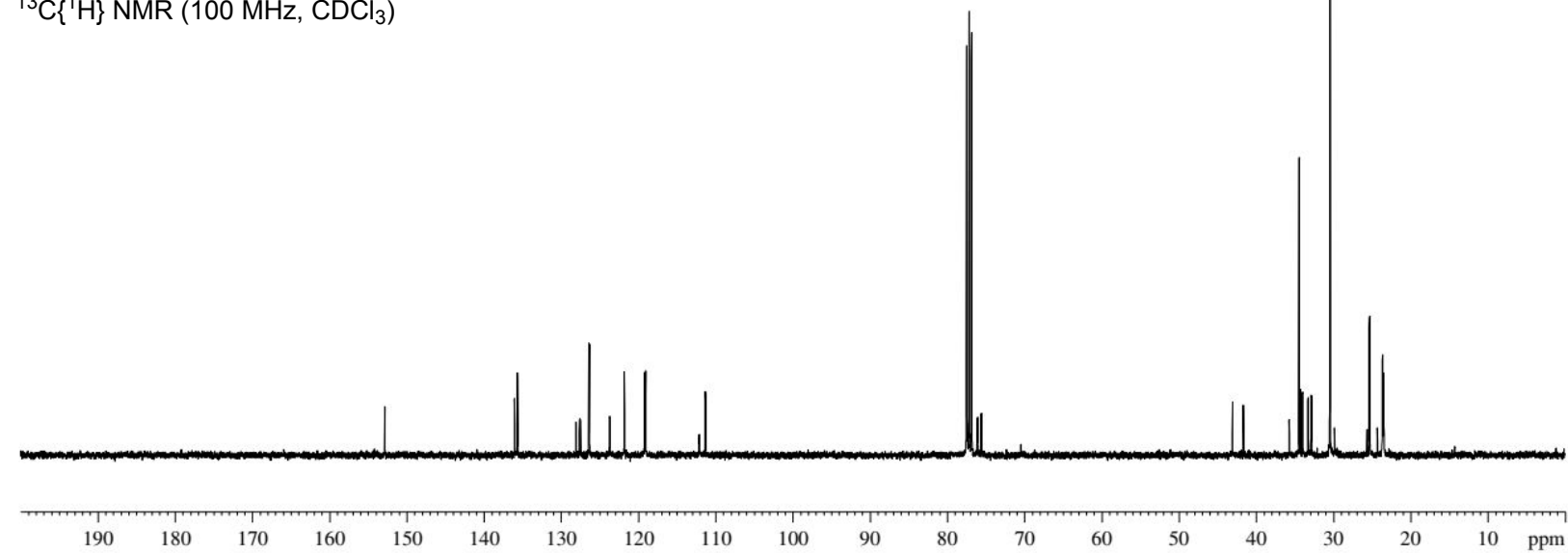




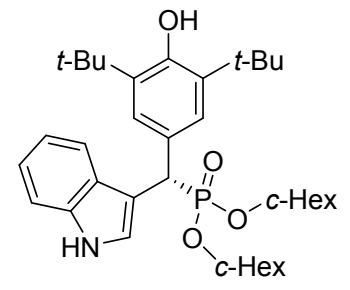

3b

${ }^{31} \mathrm{P}-\mathrm{NMR}\left(162 \mathrm{MHz}, \mathrm{CDCl}_{3}\right.$ )

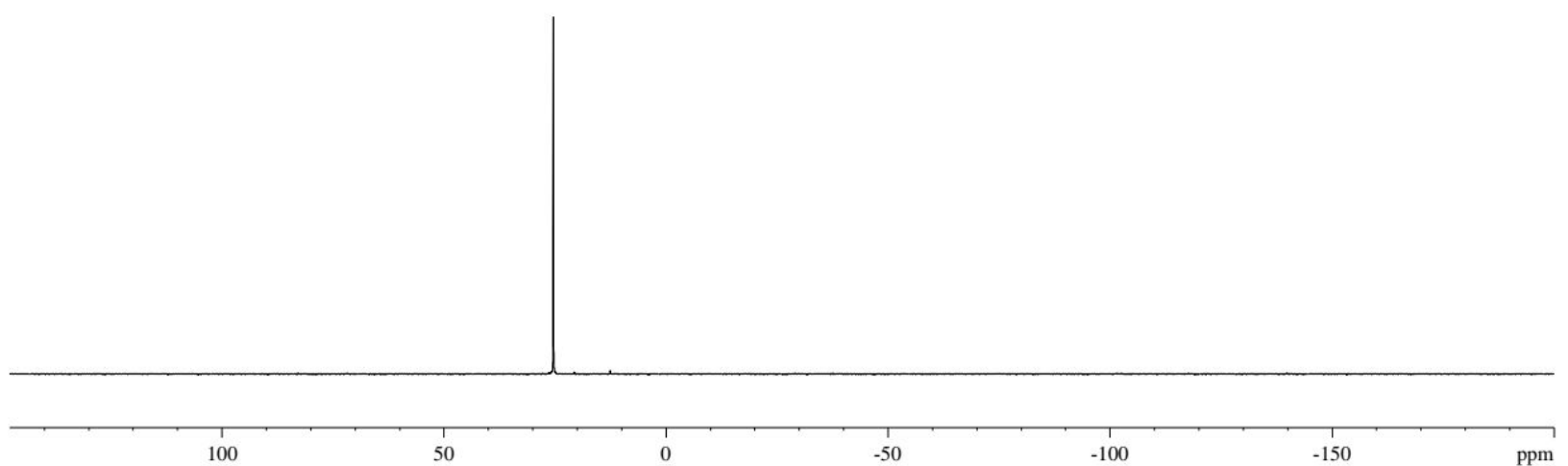

mAU

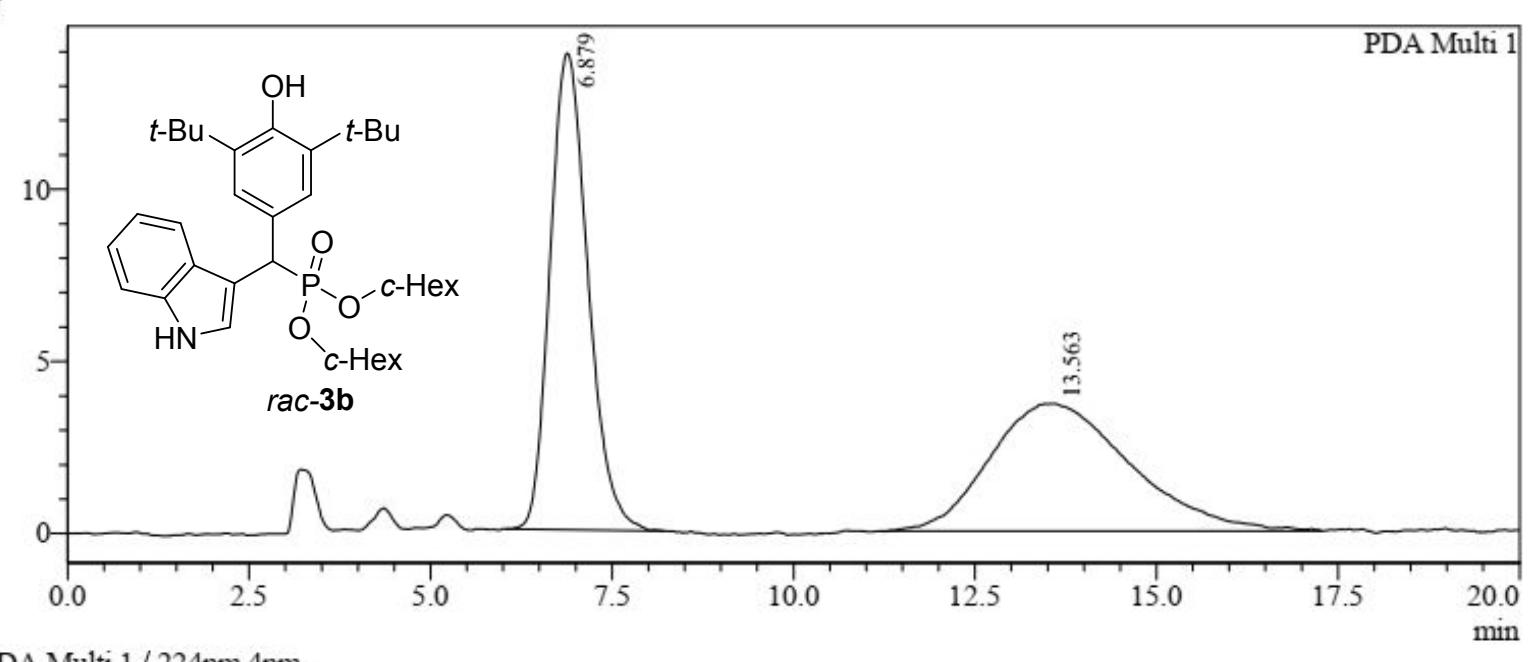

1 PDA Multi $1 / 224 \mathrm{~nm} 4 \mathrm{~nm}$

PeakTable

Ch1 224nm 4nm
\begin{tabular}{|r|r|r|r|r|}
\hline \multicolumn{1}{|c|}{ Peak\# } & Ret. Time & \multicolumn{1}{c|}{ Area } & Height & Area $\%$ \\
\hline 1 & 6.879 & 503786 & 13841 & 50.351 \\
\hline 2 & 13.563 & 496756 & 3702 & 49.649 \\
\hline Total & & 1000542 & 17543 & 100.000 \\
\hline
\end{tabular}


mAU

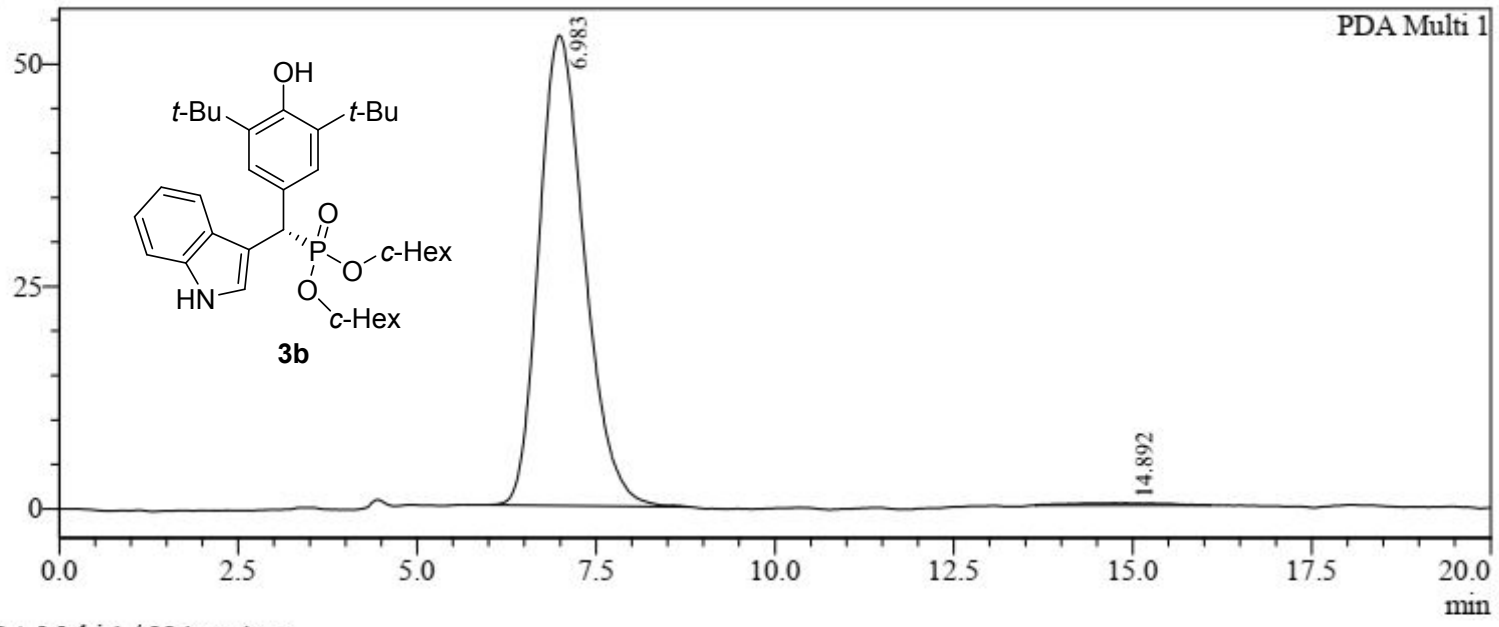

1 PDA Multi $1 / 224 \mathrm{~nm} 4 \mathrm{~nm}$

Ch1 224nm 4nm
\begin{tabular}{|r|r|r|r|r|}
\hline \multicolumn{1}{|c|}{ Peak\# } & ReakTable \\
\hline 1 & 6.983 & \multicolumn{1}{|c|}{ Area } & Height & Area $\%$ \\
\hline 2 & 14.892 & 2344545 & 52858 & 99.119 \\
\hline Total & & 20842 & 238 & 0.881 \\
\hline
\end{tabular}<smiles>CC(C)(C)c1cc(C(c2c[nH]c3ccccc23)P(=O)(OC(=O)O)OC(=O)O)cc(C(C)(C)C)c1O</smiles>

${ }^{1} \mathrm{H}-\mathrm{NMR}\left(400 \mathrm{MHz}, \mathrm{CDCl}_{3}\right)$

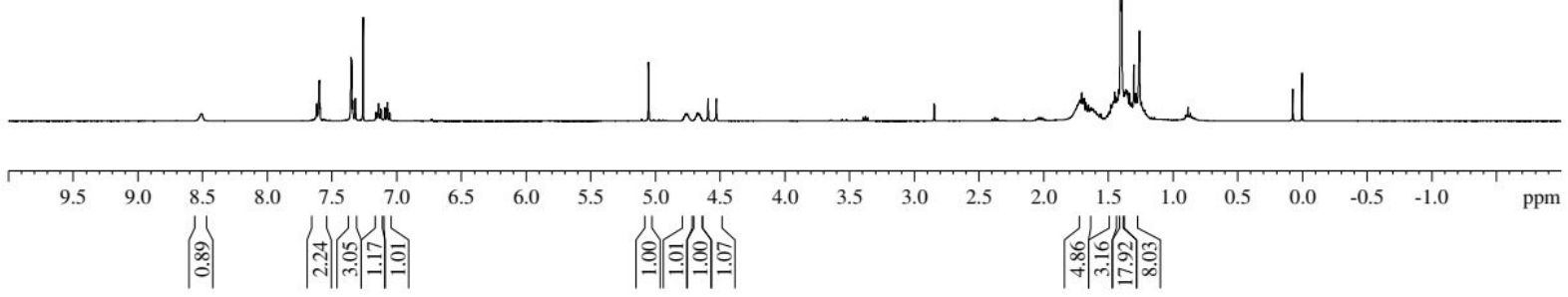



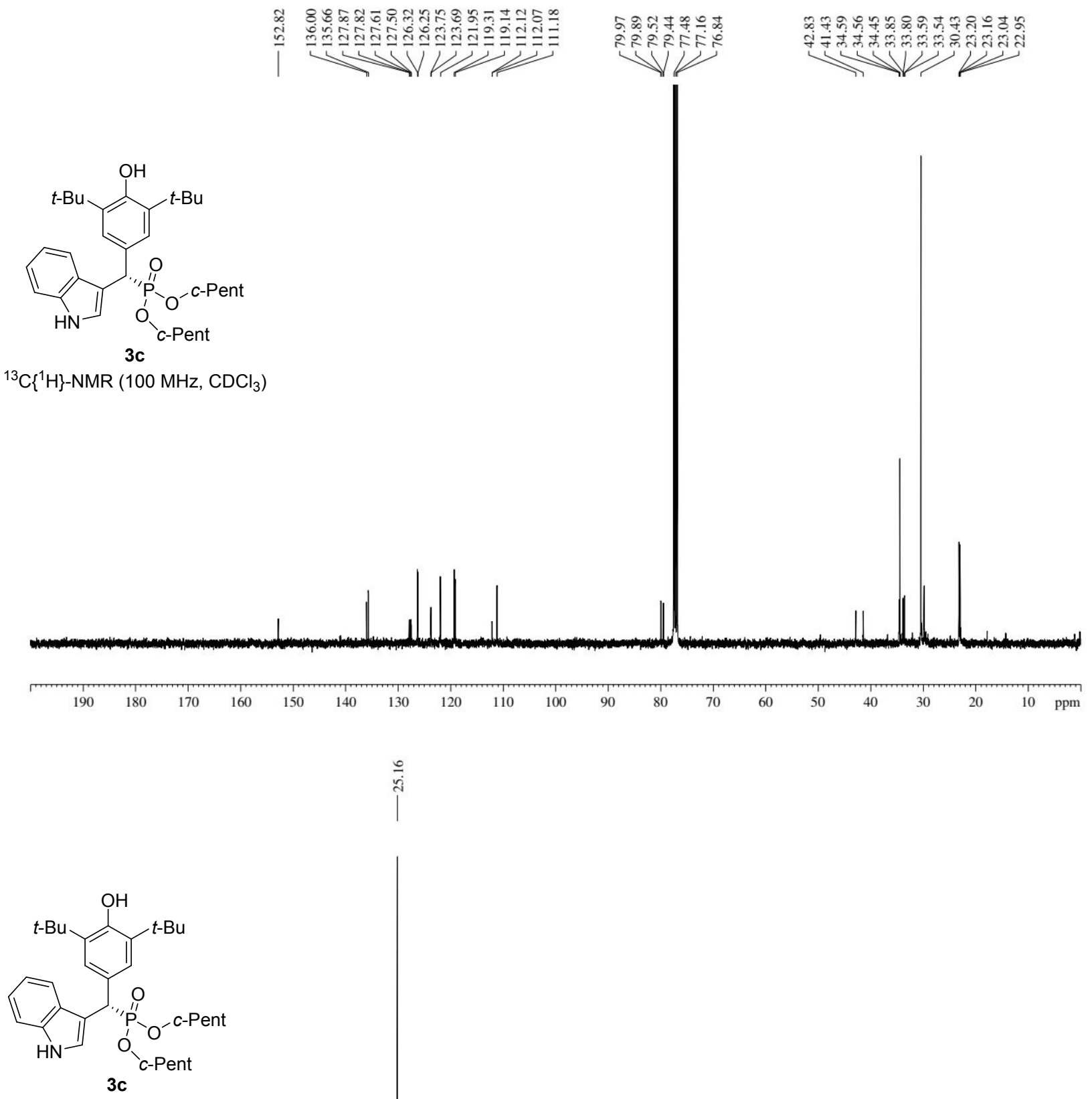

${ }^{31} \mathrm{P}-\mathrm{NMR}\left(162 \mathrm{MHz}, \mathrm{CDCl}_{3}\right)$

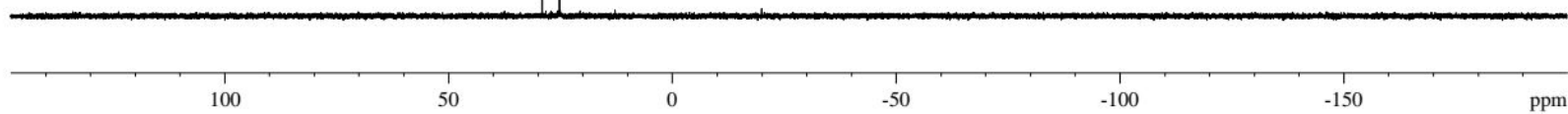


mAU

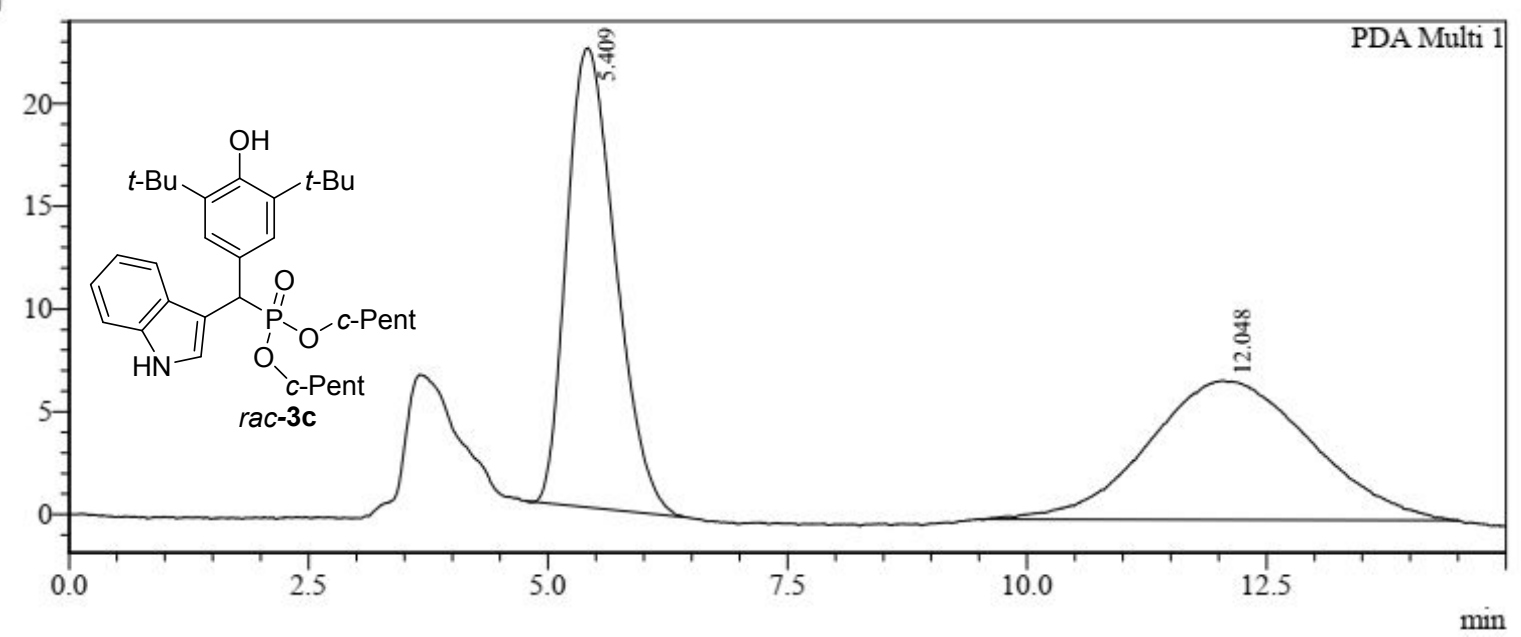

1 PDA Multi $1 / 224 \mathrm{~nm} 4 \mathrm{~nm}$

Ch1 224nm 4nm
\begin{tabular}{|r|r|r|r|r|}
\hline \multicolumn{1}{|c|}{ PeakT\# } & Ret. Time & Area & Height & \multicolumn{1}{c|}{ Area $\%$} \\
\hline 1 & 5.409 & 795708 & 22360 & 49.764 \\
\hline 2 & 12.048 & 803261 & 6789 & 50.236 \\
\hline Total & & 1598969 & 29149 & 100.000 \\
\hline
\end{tabular}

mAU

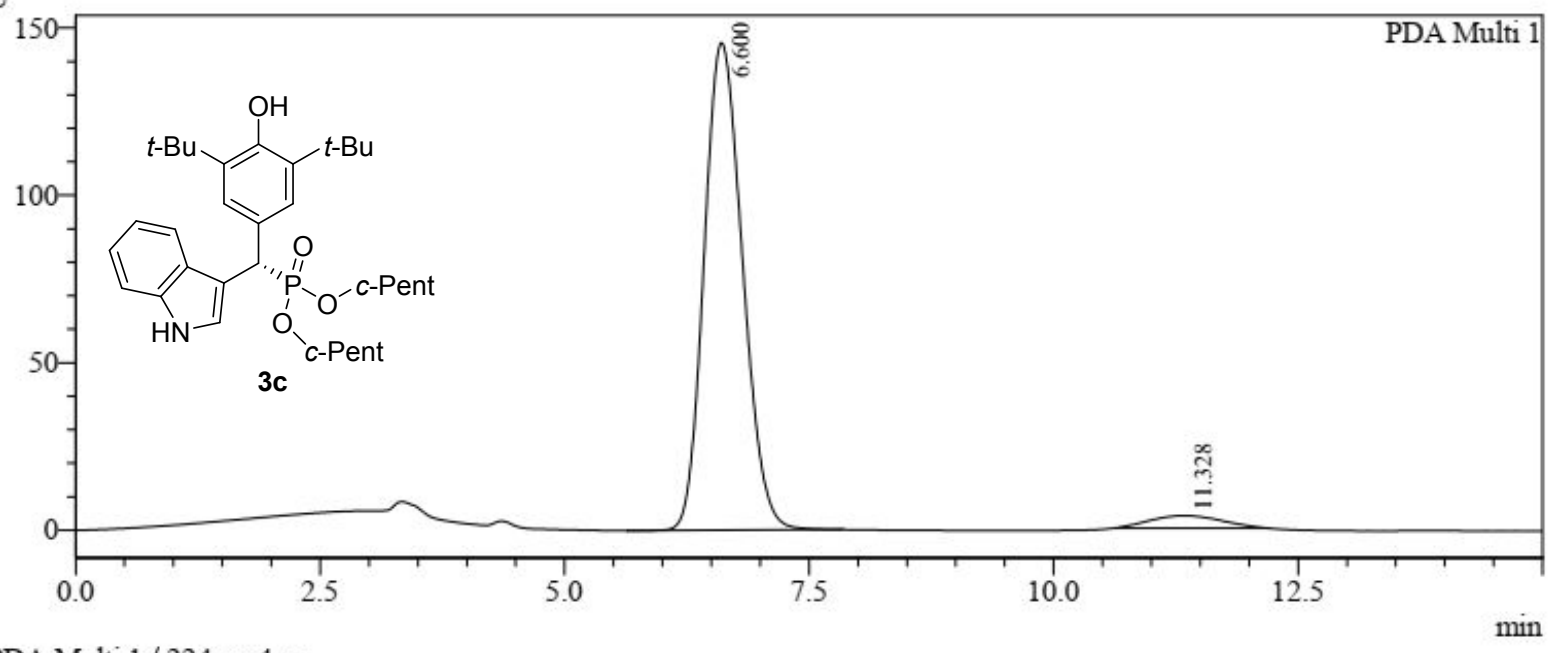

1 PDA Multi $1 / 224 \mathrm{~nm} 4 \mathrm{~nm}$

PeakTable

Ch1 224nm 4nm
\begin{tabular}{|r|r|r|r|r|}
\hline Peak\# & Ret. Time & \multicolumn{1}{|c|}{ Area } & Height & Area \% \\
\hline 1 & 6.600 & 3983232 & 145587 & 95.407 \\
\hline 2 & 11.328 & 191769 & 3709 & 4.593 \\
\hline Total & & 4175001 & 149296 & 100.000 \\
\hline
\end{tabular}



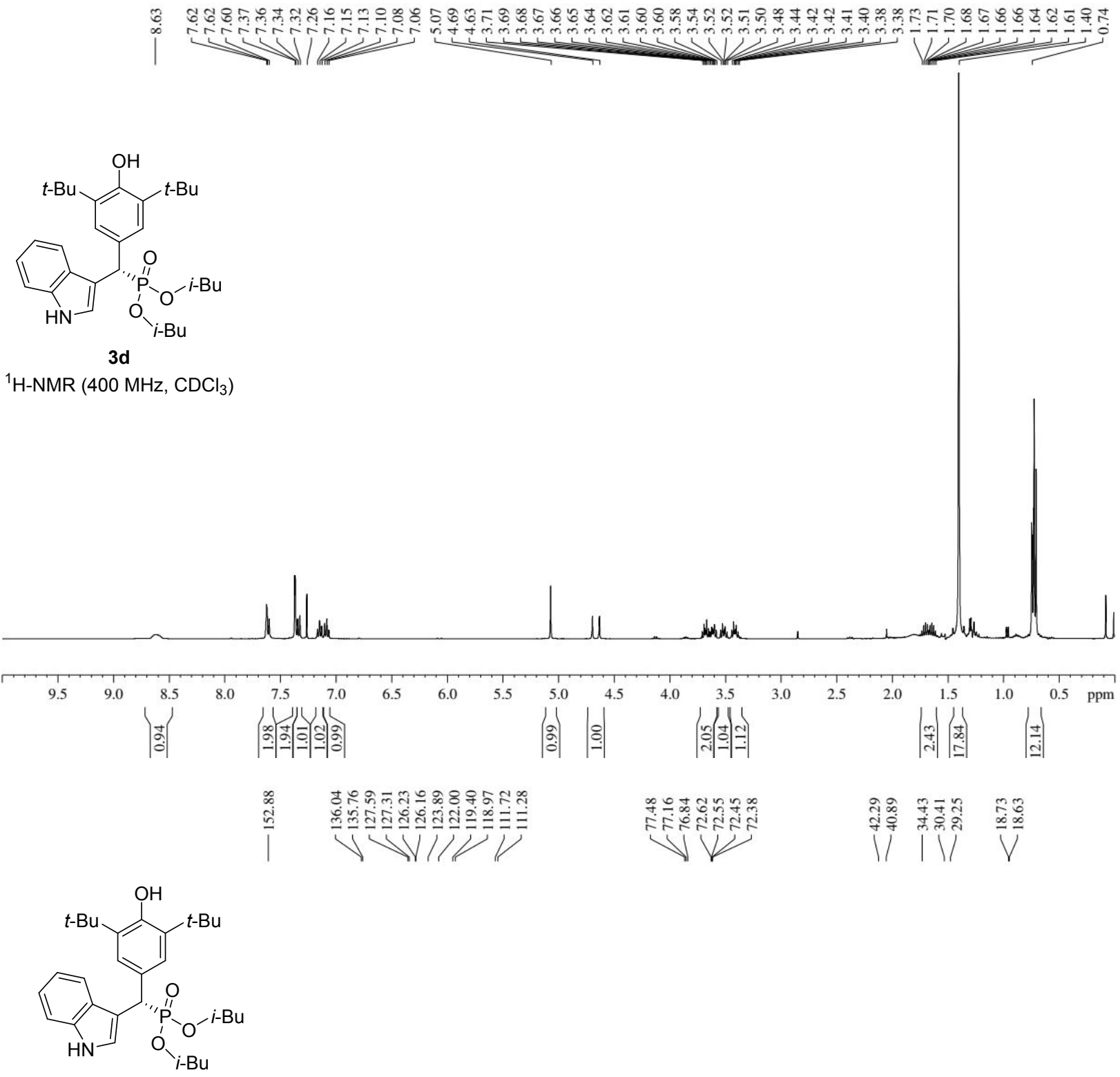

3d

${ }^{13} \mathrm{C}\left\{{ }^{1} \mathrm{H}\right\}-\mathrm{NMR}\left(100 \mathrm{MHz}, \mathrm{CDCl}_{3}\right)$

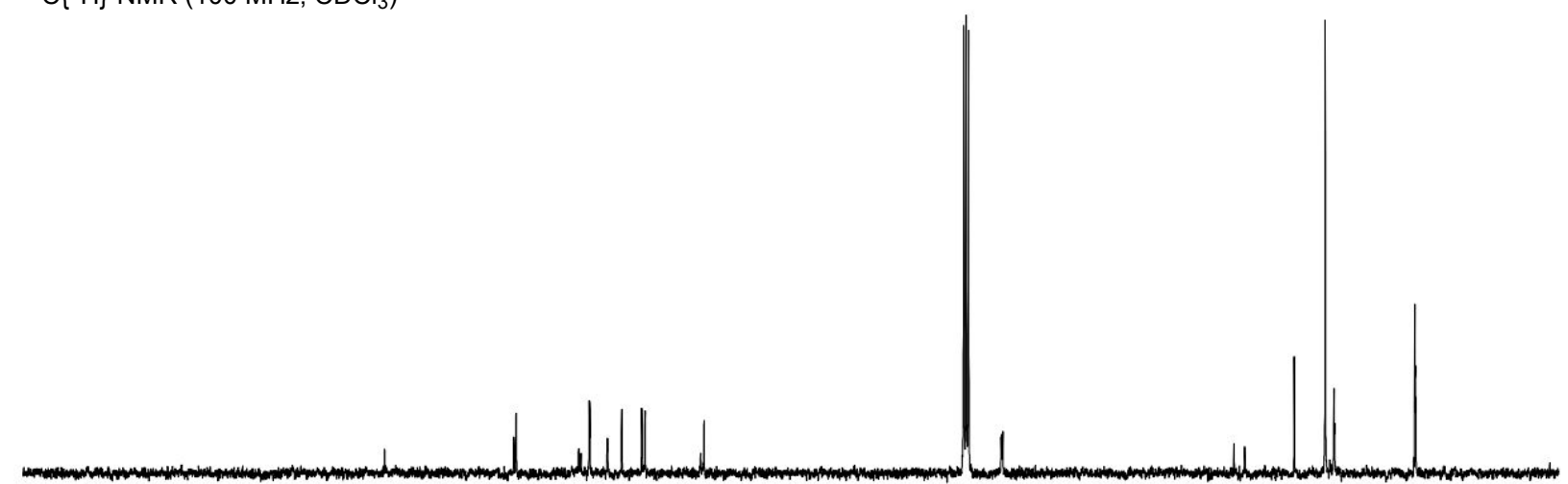

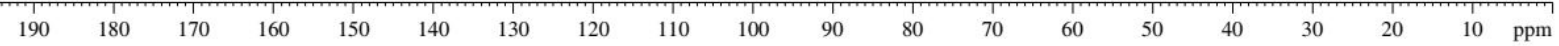




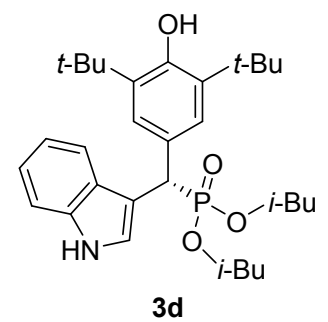

${ }^{31} \mathrm{P}-\mathrm{NMR}\left(162 \mathrm{MHz}, \mathrm{CDCl}_{3}\right)$

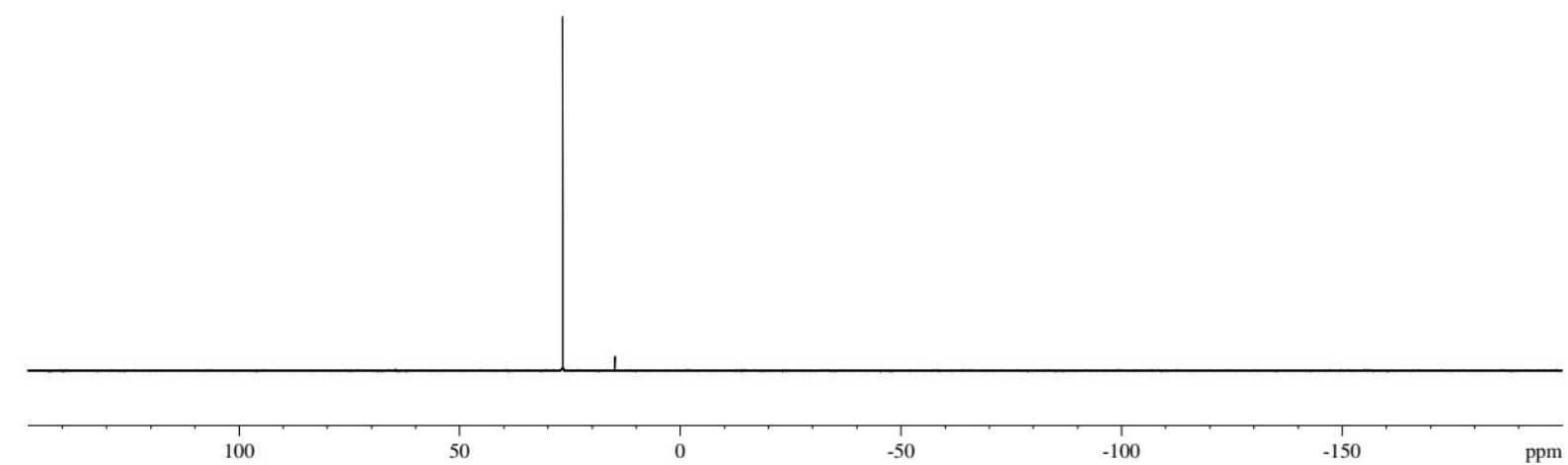

mAU

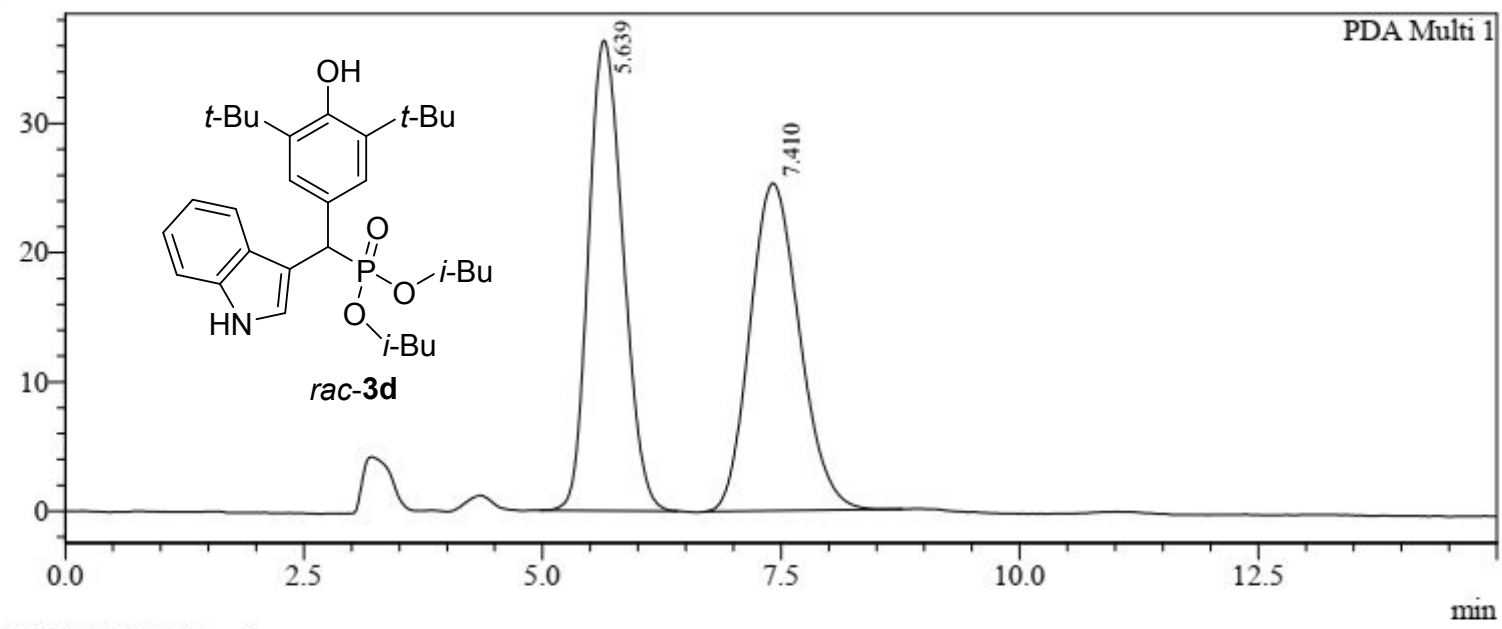

1 PDA Multi $1 / 224 \mathrm{~nm} 4 \mathrm{~nm}$

\begin{tabular}{|c|c|c|c|c|}
\hline \multicolumn{5}{|c|}{$\mathrm{Ch} 1224 \mathrm{~nm} 4 \mathrm{~nm}$} \\
\hline Peak\# & Ret. Time & Area & Height & Area \% \\
\hline 1 & 5.639 & 920888 & 36398 & 50.124 \\
\hline 2 & 7.410 & 916342 & 25355 & 49.876 \\
\hline Total & & 1837230 & 61753 & 100.000 \\
\hline
\end{tabular}


mAU

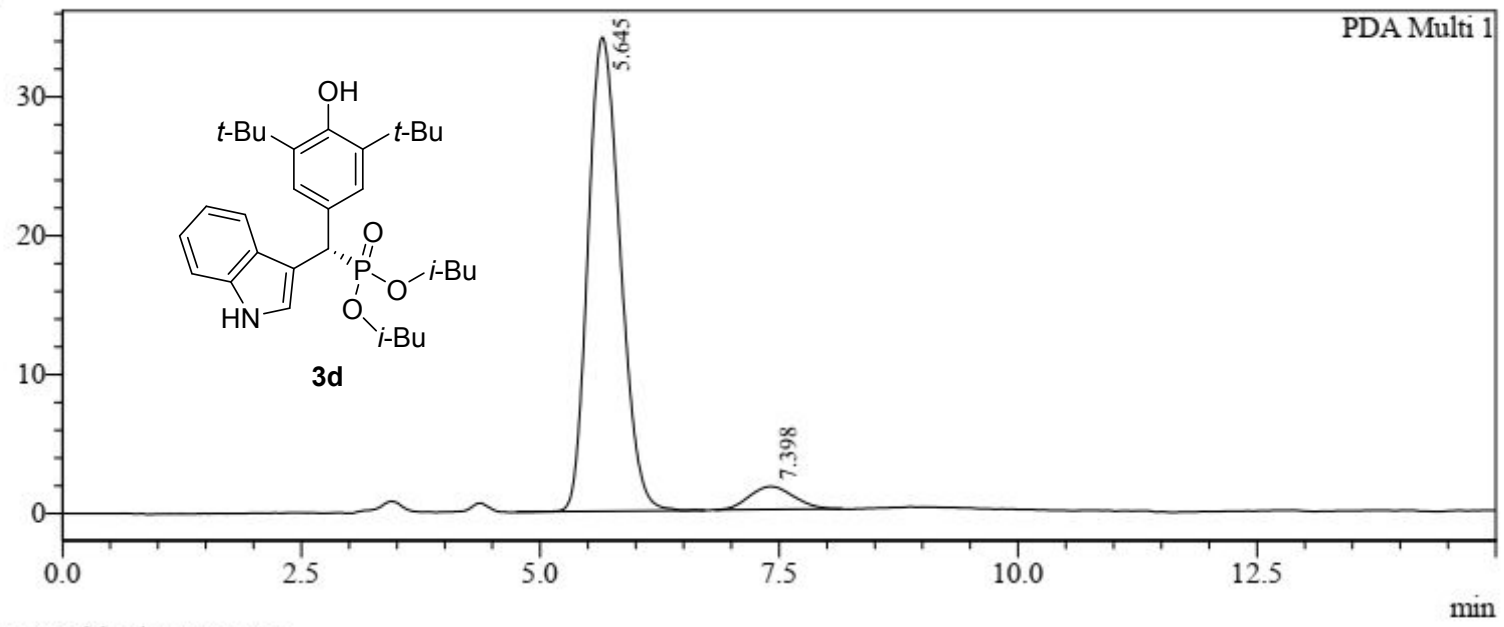

1 PDA Multi $1 / 224 \mathrm{~nm} 4 \mathrm{~nm}$

Ch1 224nm 4nm
\begin{tabular}{|r|r|r|r|r|}
\hline \multicolumn{1}{|c|}{ Peak\# } & Ret. Time & Area & Height & \multicolumn{1}{c|}{ Area \% } \\
\hline 1 & 5.645 & 800758 & 34132 & 93.720 \\
\hline 2 & 7.398 & 53660 & 1659 & 6.280 \\
\hline Total & & 854418 & 35790 & 100.000 \\
\hline
\end{tabular}

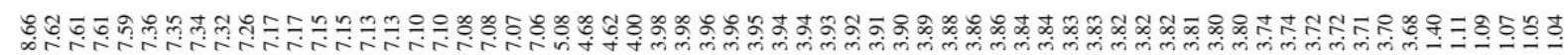<smiles>CCOP(=O)(OC)C(c1cc(C(C)(C)C)c(O)c(C(C)(C)C)c1)c1c[nH]c2ccccc12</smiles>

${ }^{1} \mathrm{H}-\mathrm{NMR}\left(400 \mathrm{MHz}, \mathrm{CDCl}_{3}\right)$

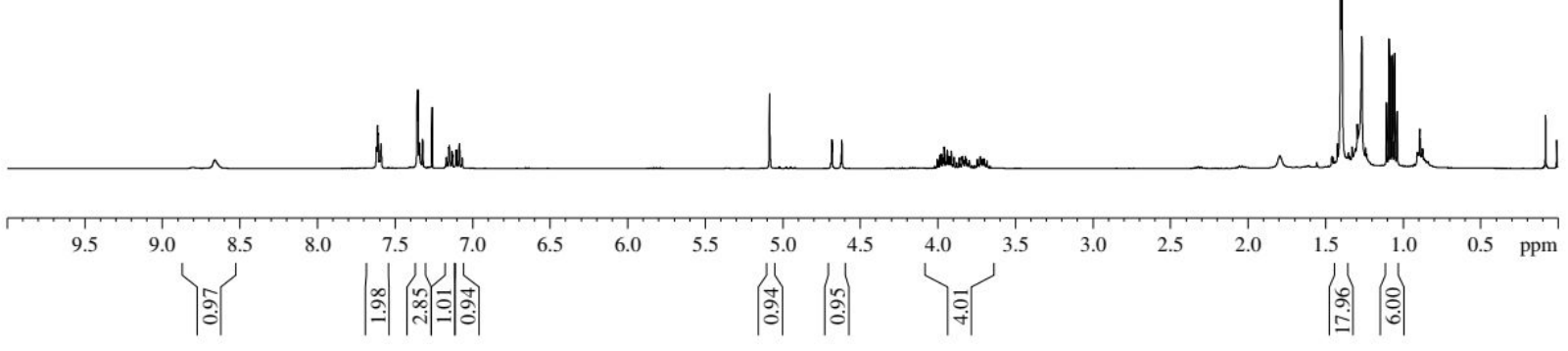


Arylated-Methyl Organophosphorus Compounds, Tripathi et al., SI-Part-B, Page S-34
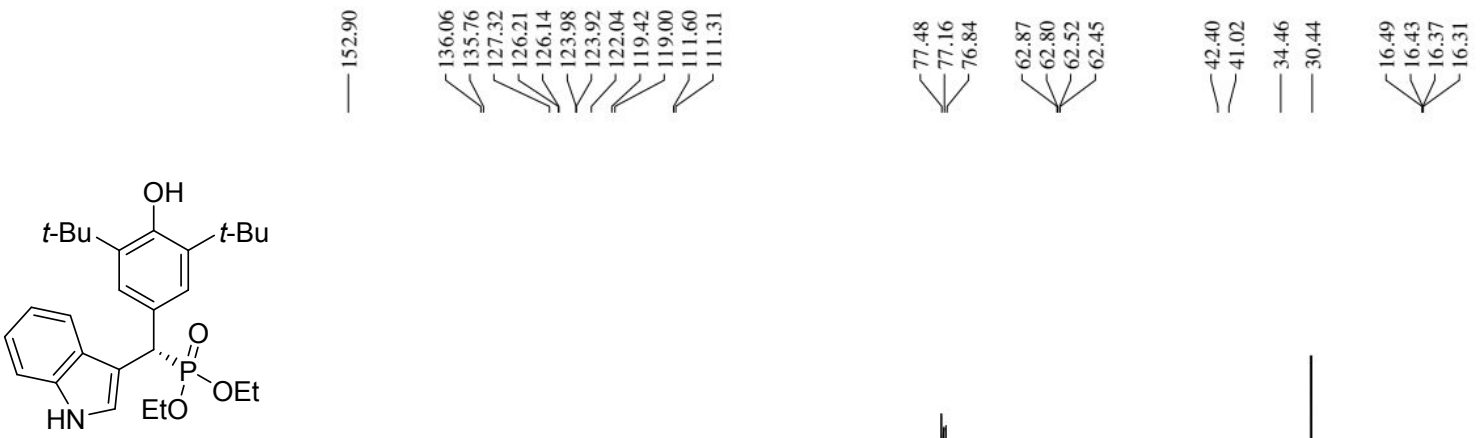

$3 e$

${ }^{13} \mathrm{C}\left\{{ }^{1} \mathrm{H}\right\}-N M R\left(100 \mathrm{MHz}, \mathrm{CDCl}_{3}\right)$
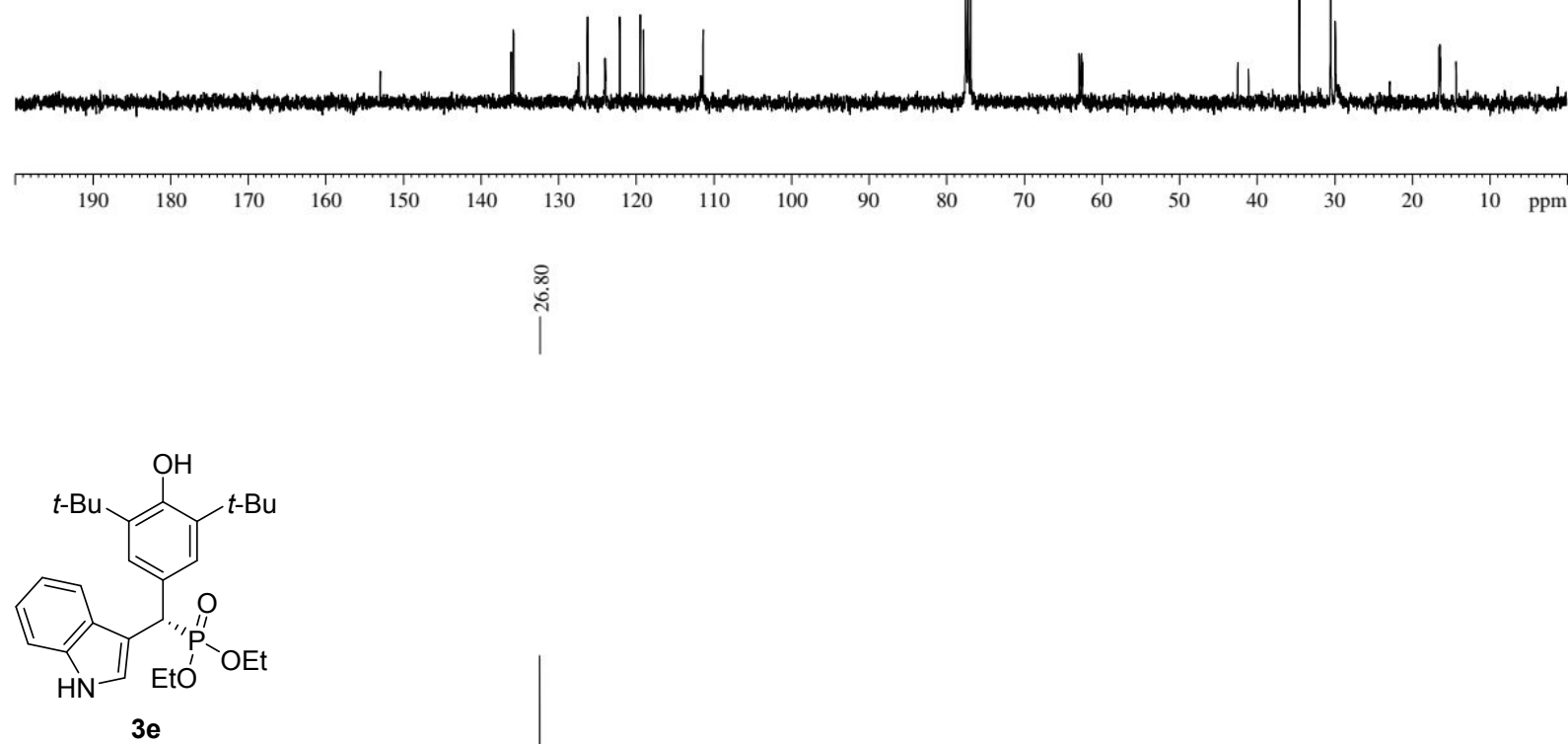

${ }^{31} \mathrm{P}-\mathrm{NMR}\left(162 \mathrm{MHz}, \mathrm{CDCl}_{3}\right.$ )

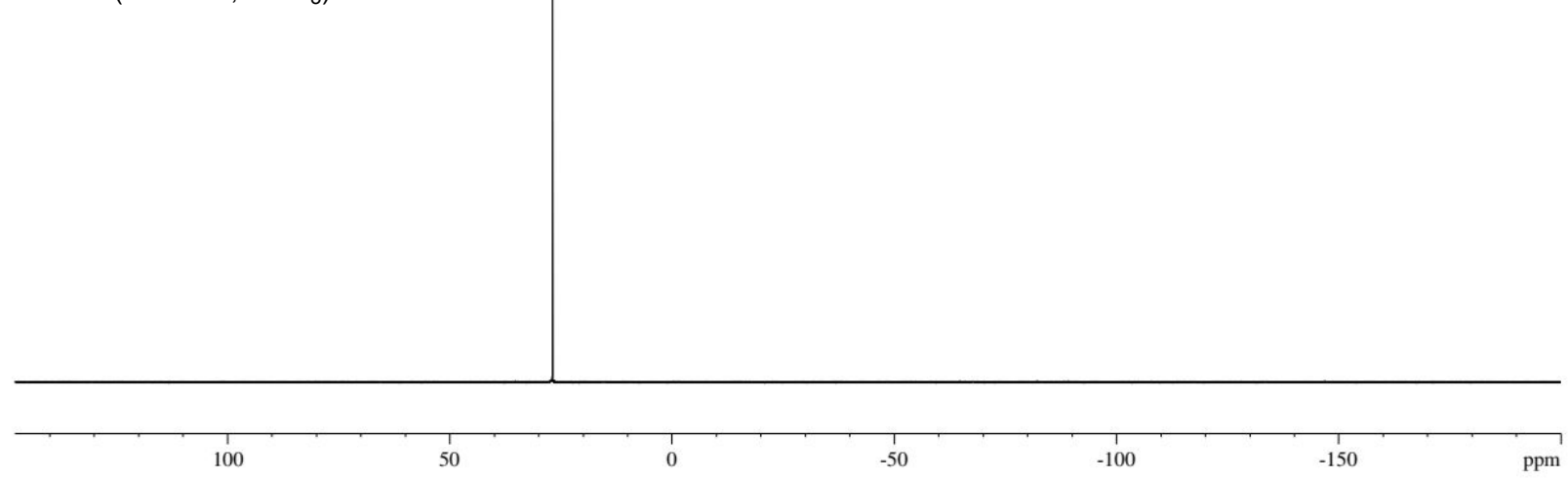


mAU

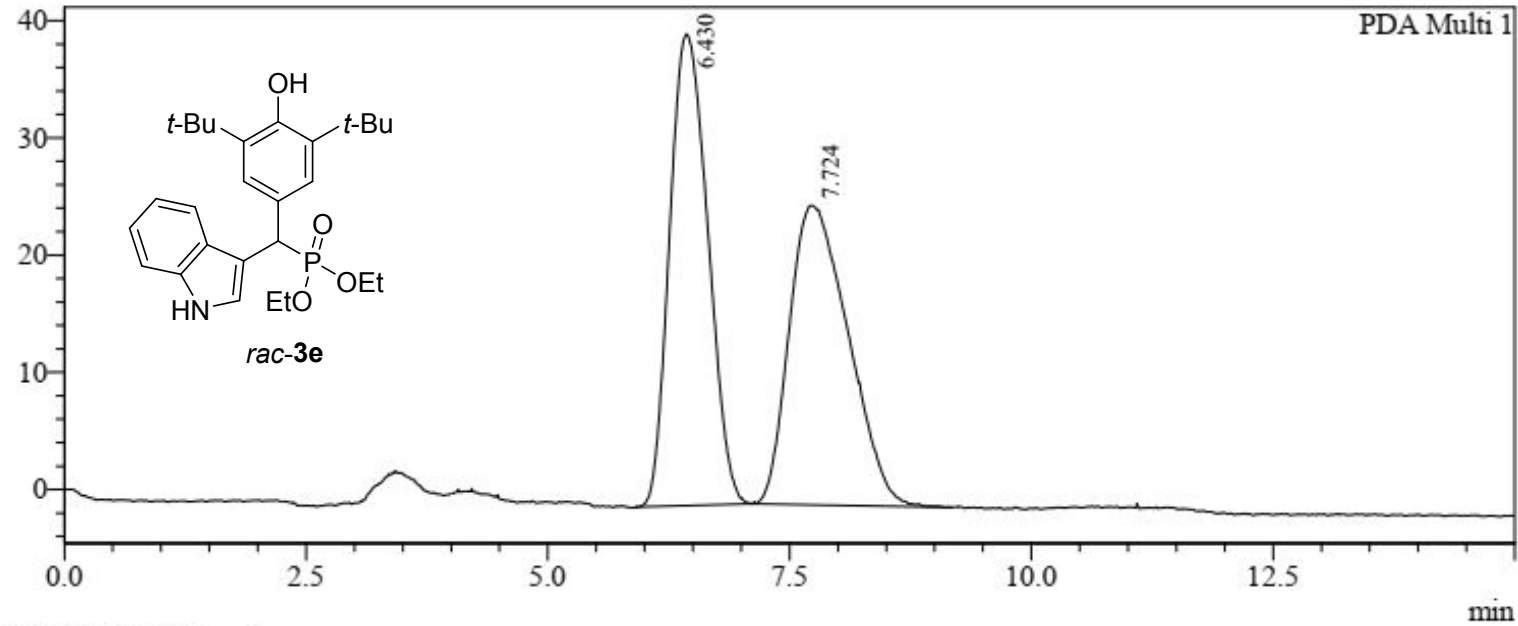

1 PDA Multi $1 / 224 \mathrm{~nm} 4 \mathrm{~nm}$

Ch1 224nm 4nm
\begin{tabular}{|r|r|r|r|r|}
\hline \multicolumn{1}{|c|}{ Peak \# } & Ret. Time & Area & Height & \multicolumn{1}{|c|}{ Area \% } \\
\hline 1 & 6.430 & 1105932 & 40215 & 50.518 \\
\hline 2 & 7.724 & 1083261 & 25537 & 49.482 \\
\hline Total & & 2189193 & 65752 & 100.000 \\
\hline
\end{tabular}

mAU

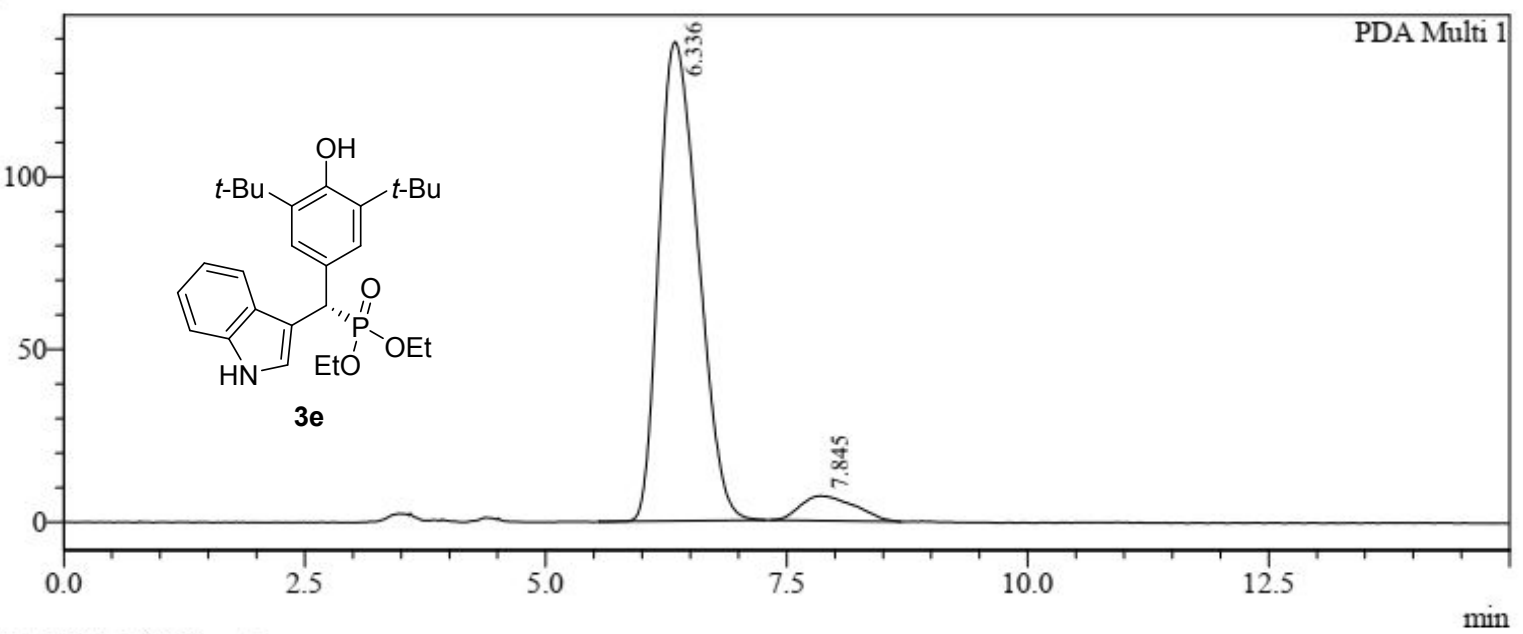

1 PDA Multi 1/224nm 4nm

Ch1 224nm 4nm
\begin{tabular}{|r|r|r|r|r|}
\hline \multicolumn{1}{|c|}{ Peak\# } & Ret. Time & \multicolumn{1}{c|}{ Area } & Height & \multicolumn{1}{|c|}{ Area $\%$} \\
\hline 1 & 6.336 & 3947628 & 138737 & 93.323 \\
\hline 2 & 7.845 & 282424 & 7194 & 6.677 \\
\hline Total & & 4230052 & 145931 & 100.000 \\
\end{tabular}




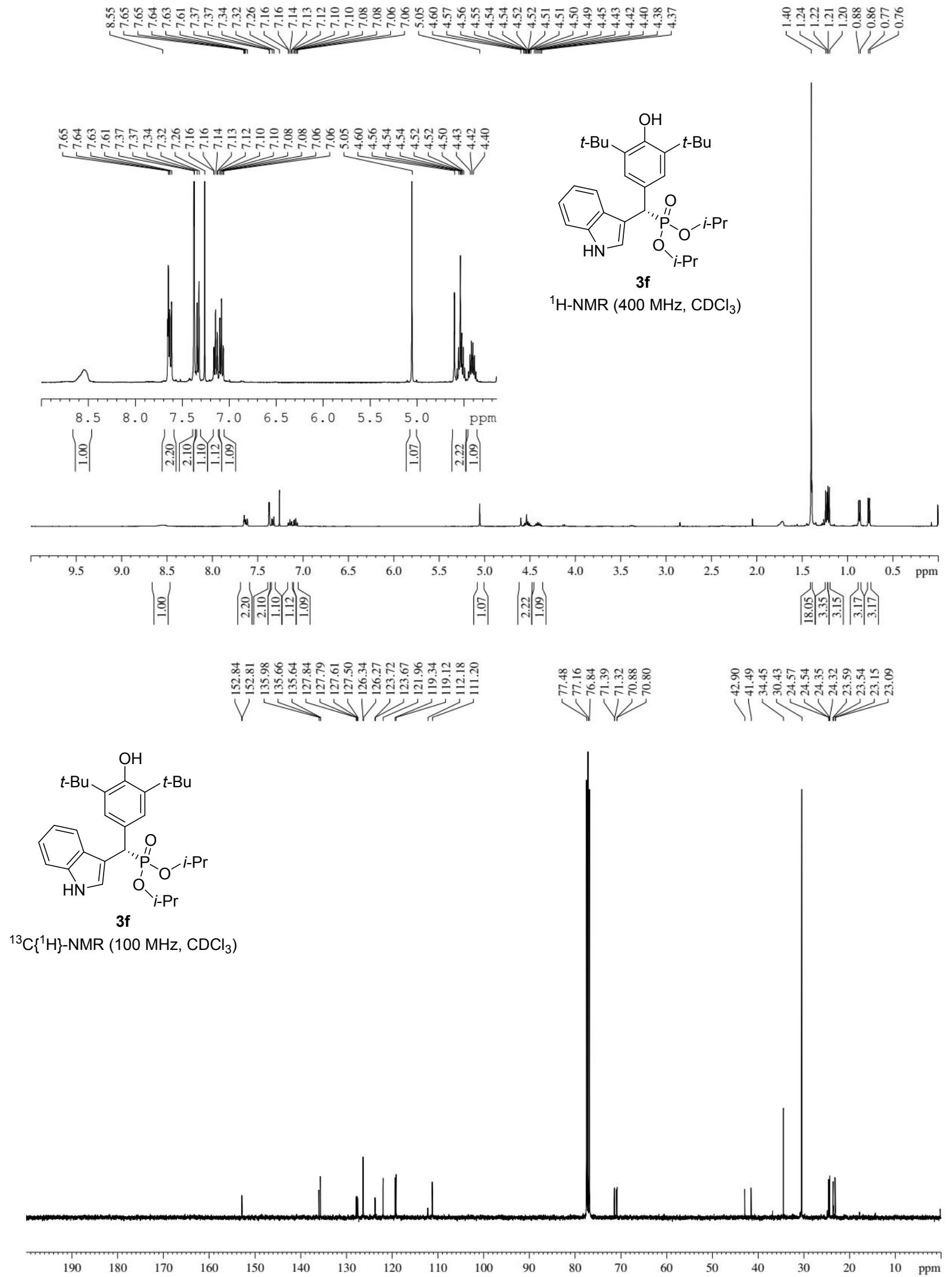



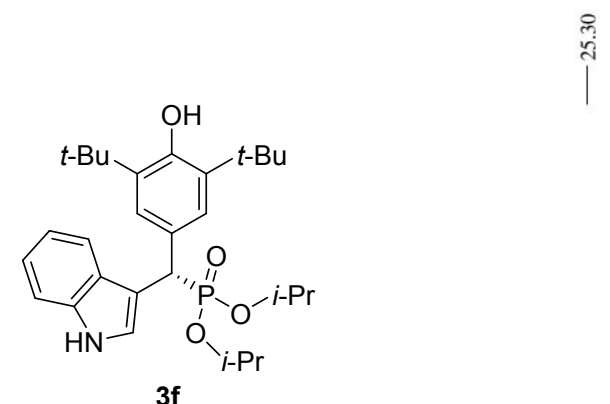

${ }^{31} \mathrm{P}-\mathrm{NMR}\left(162 \mathrm{MHz}, \mathrm{CDCl}_{3}\right.$ )

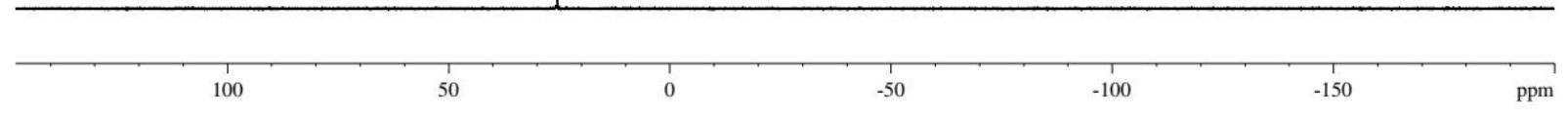

mAU

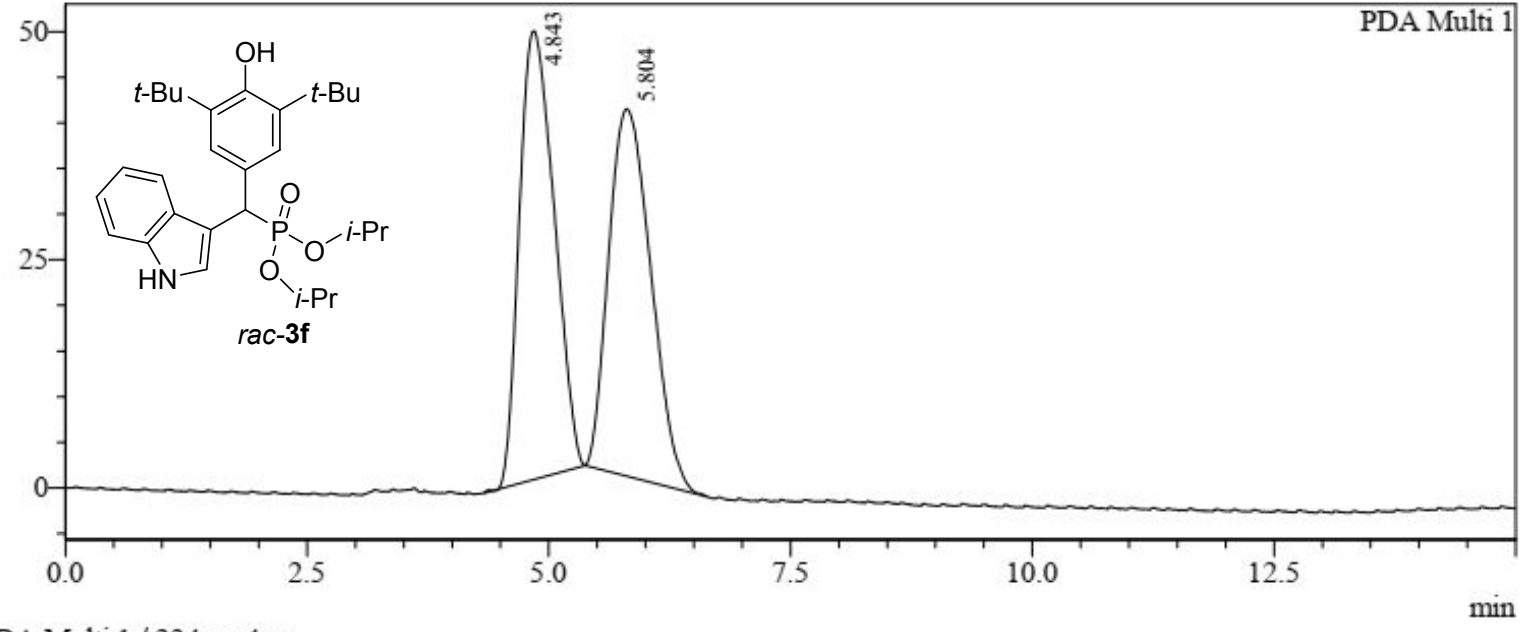

1 PDA Multi $1 / 224 \mathrm{~nm} 4 \mathrm{~nm}$

PeakTable

Ch1 224nm 4nm
\begin{tabular}{|r|r|r|r|r|}
\hline \multicolumn{1}{|c|}{ Peak\# } & Ret. Time & Area & Height & Area \% \\
\hline 1 & 4.843 & 1233447 & 49141 & 50.222 \\
\hline 2 & 5.804 & 1222546 & 40246 & 49.778 \\
\hline Total & & 2455993 & 89386 & 100.000 \\
\hline
\end{tabular}


mAU

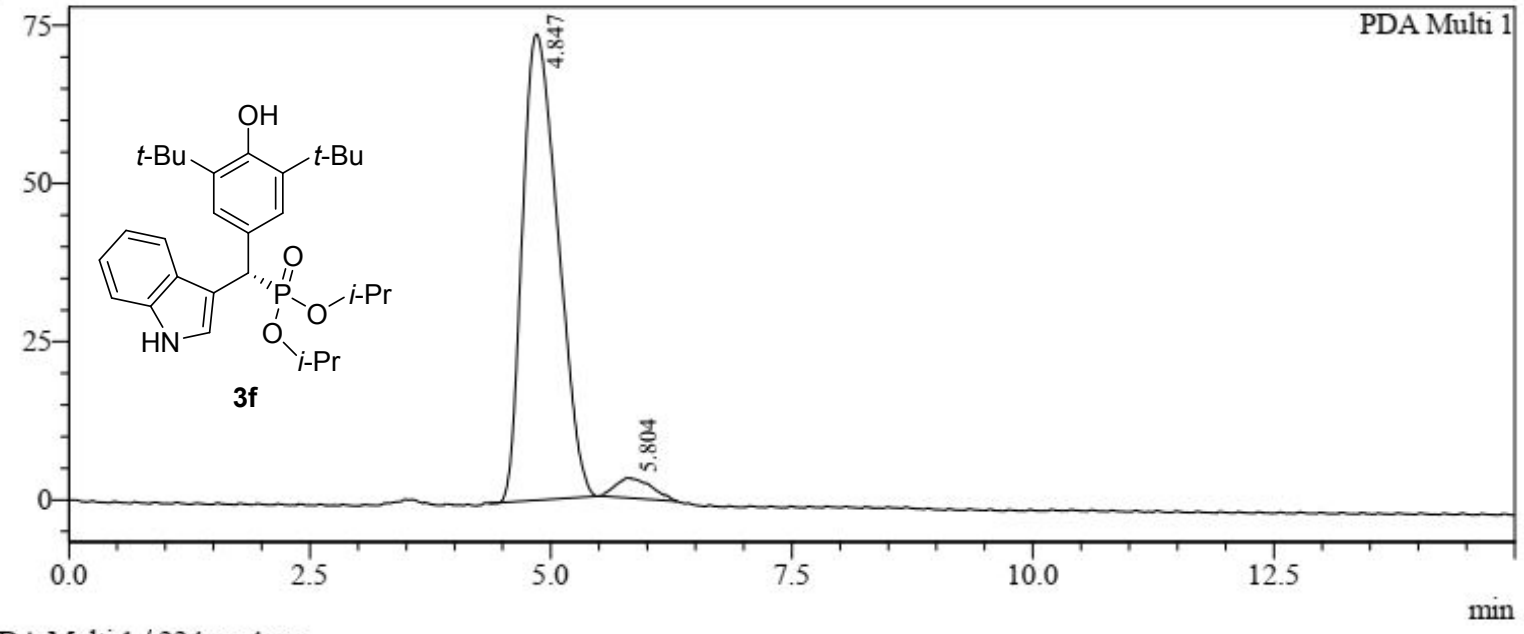

1 PDA Multi $1 / 224 \mathrm{~nm} 4 \mathrm{~nm}$

PeakTable

Ch1 224nm 4nm
\begin{tabular}{|r|r|r|r|r|}
\multicolumn{1}{|c|}{ PeakTable } \\
\hline 1 & Ret. Time & \multicolumn{1}{|c|}{ Area } & Height & \multicolumn{1}{|c|}{ Area $\%$} \\
\hline 2 & 4.847 & 1885224 & 73691 & 95.806 \\
\hline Total & 5.804 & 82526 & 3157 & 4.194 \\
\hline & & 1967750 & 76848 & 100.000 \\
\hline
\end{tabular}

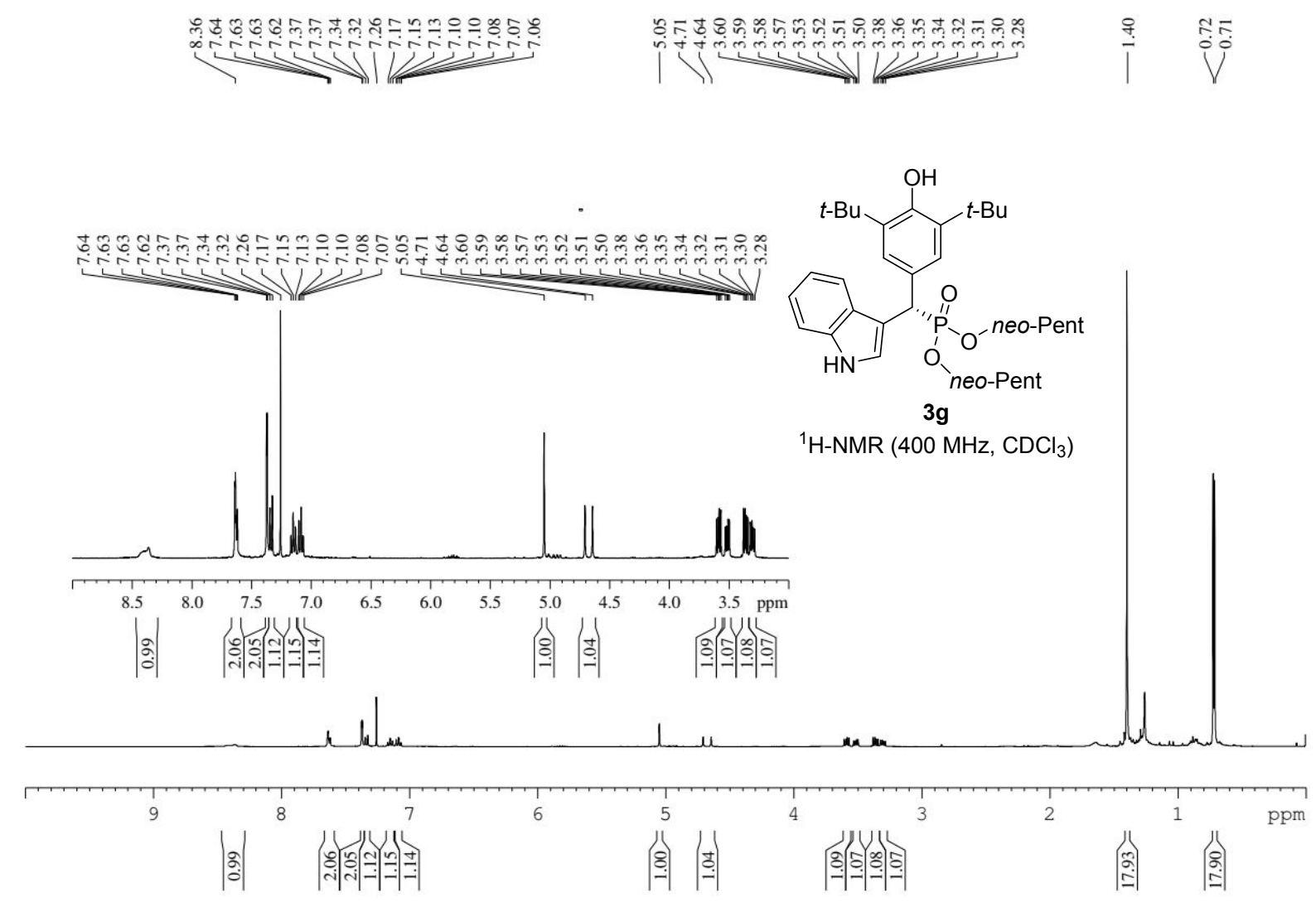



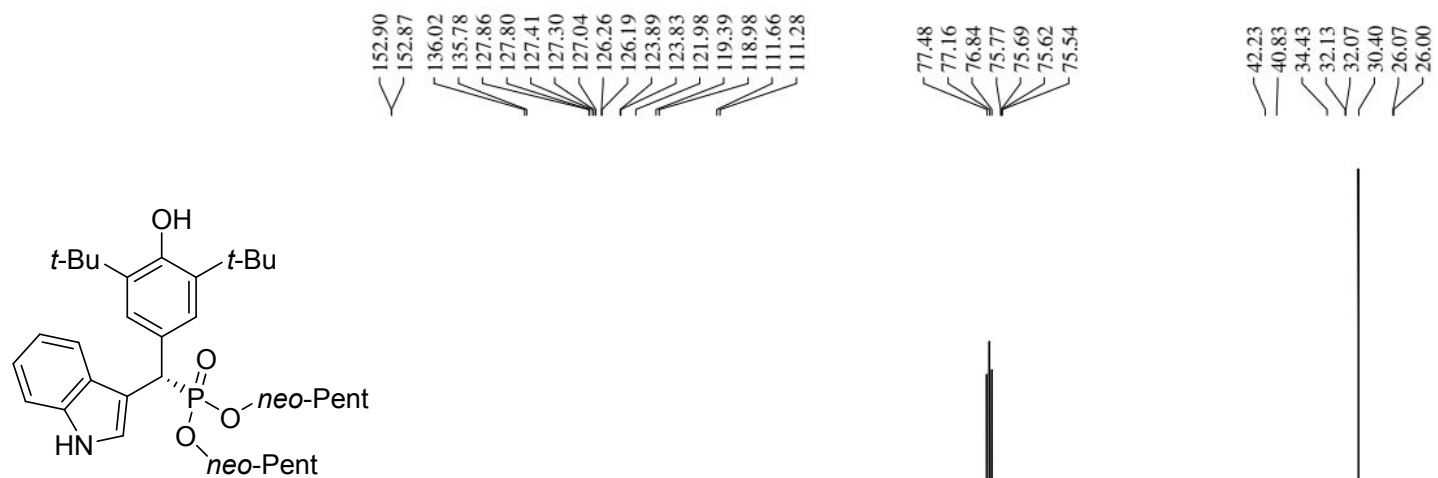

$3 \mathrm{~g}$

${ }^{13} \mathrm{C}\left\{{ }^{1} \mathrm{H}\right\}-\mathrm{NMR}\left(100 \mathrm{MHz}, \mathrm{CDCl}_{3}\right)$

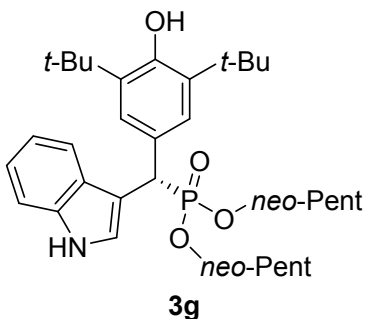

|

${ }^{31}$ P-NMR (162 MHz, CDCl 3 )

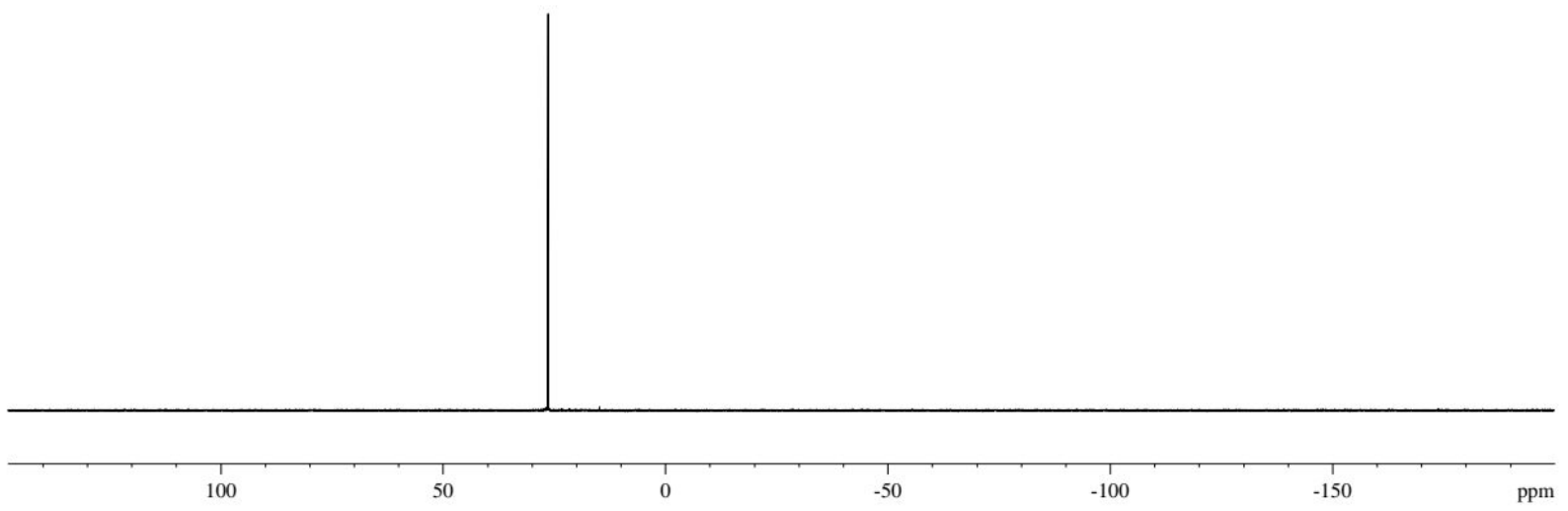


mAU

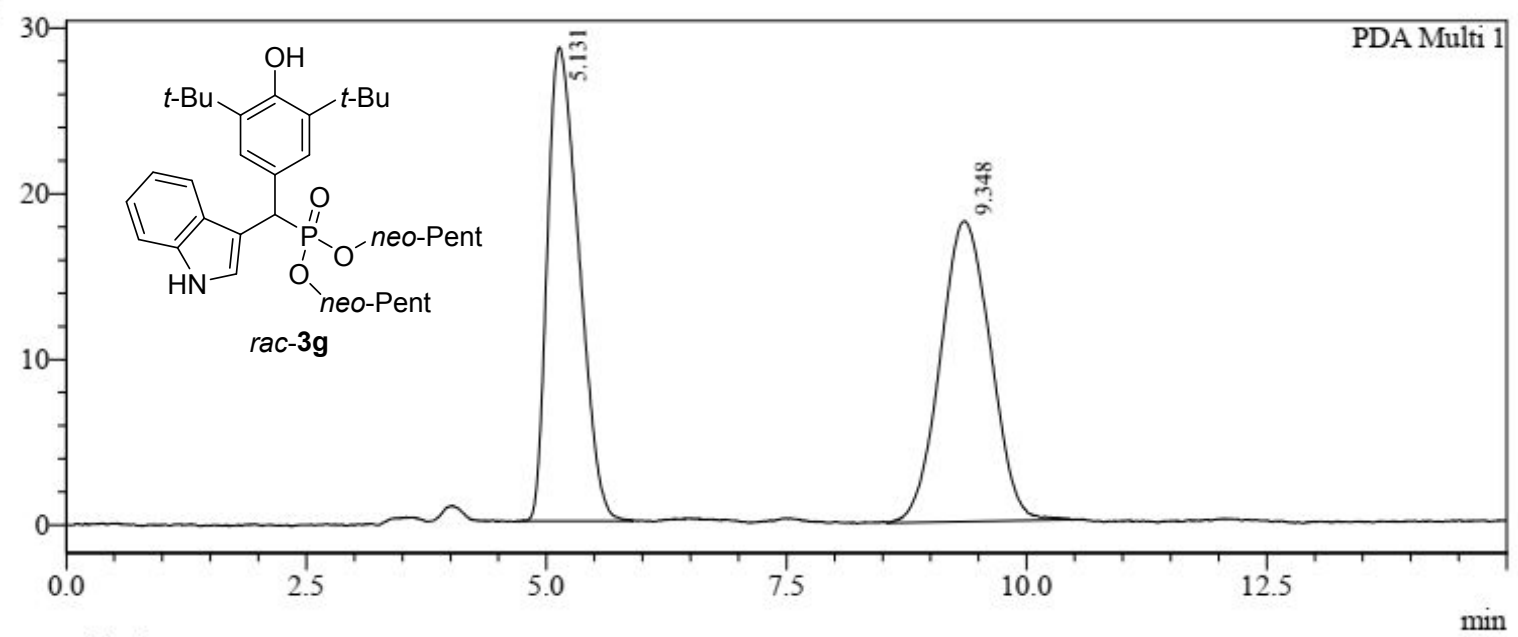

1 PDA Multi $1 / 224 \mathrm{~nm} 4 \mathrm{~nm}$

\begin{tabular}{|c|c|c|c|c|}
\hline \multicolumn{5}{|c|}{ Ch1 $224 \mathrm{~nm} 4 \mathrm{~nm}$} \\
\hline Peak\# & Ret. Time & Area & Height & Area \% \\
\hline 1 & 5.131 & 653654 & 28591 & 49.773 \\
\hline 2 & 9.348 & 659623 & 18154 & 50.227 \\
\hline Total & & 1313277 & 46745 & 100.000 \\
\hline
\end{tabular}

$\mathrm{mAU}$

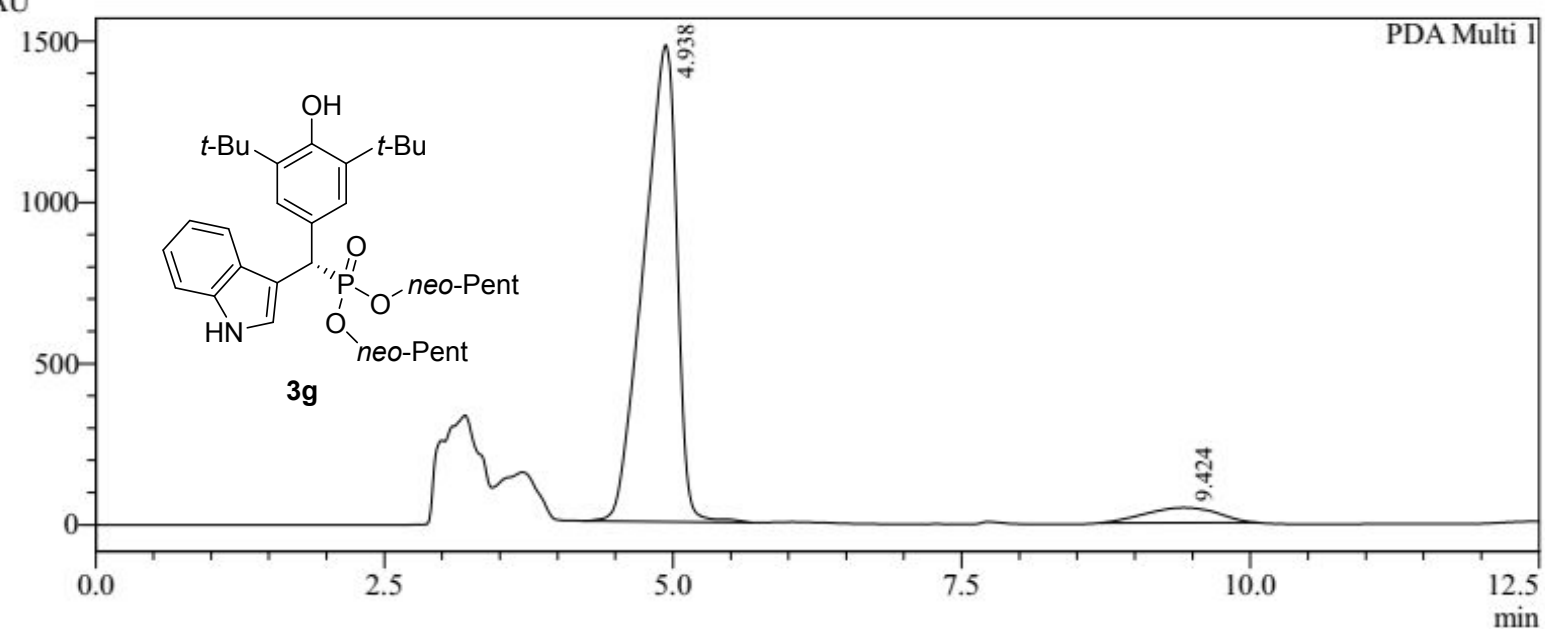

1 PDA Multi $1 / 224 \mathrm{~nm}, 4 \mathrm{~nm}$

\begin{tabular}{|c|c|c|c|c|}
\hline \multicolumn{5}{|c|}{ PDA Ch1 224nm } \\
\hline Peak\# & Ret. Time & Area & Height & Area $\%$ \\
\hline 1 & 4.938 & 29748232 & 1478051 & 93.569 \\
\hline 2 & 9.424 & 2044749 & 46518 & 6.431 \\
\hline Total & & 31792982 & 1524569 & 100.000 \\
\hline
\end{tabular}


<smiles>CCCOP(=O)(OCC(C)(C)C)C(c1cc(C(C)(C)C)c(O)c(C(C)(C)C)c1)c1c[nH]c2ccccc12</smiles>

${ }^{1} \mathrm{H}-\mathrm{NMR}\left(400 \mathrm{MHz}, \mathrm{CDCl}_{3}\right)$
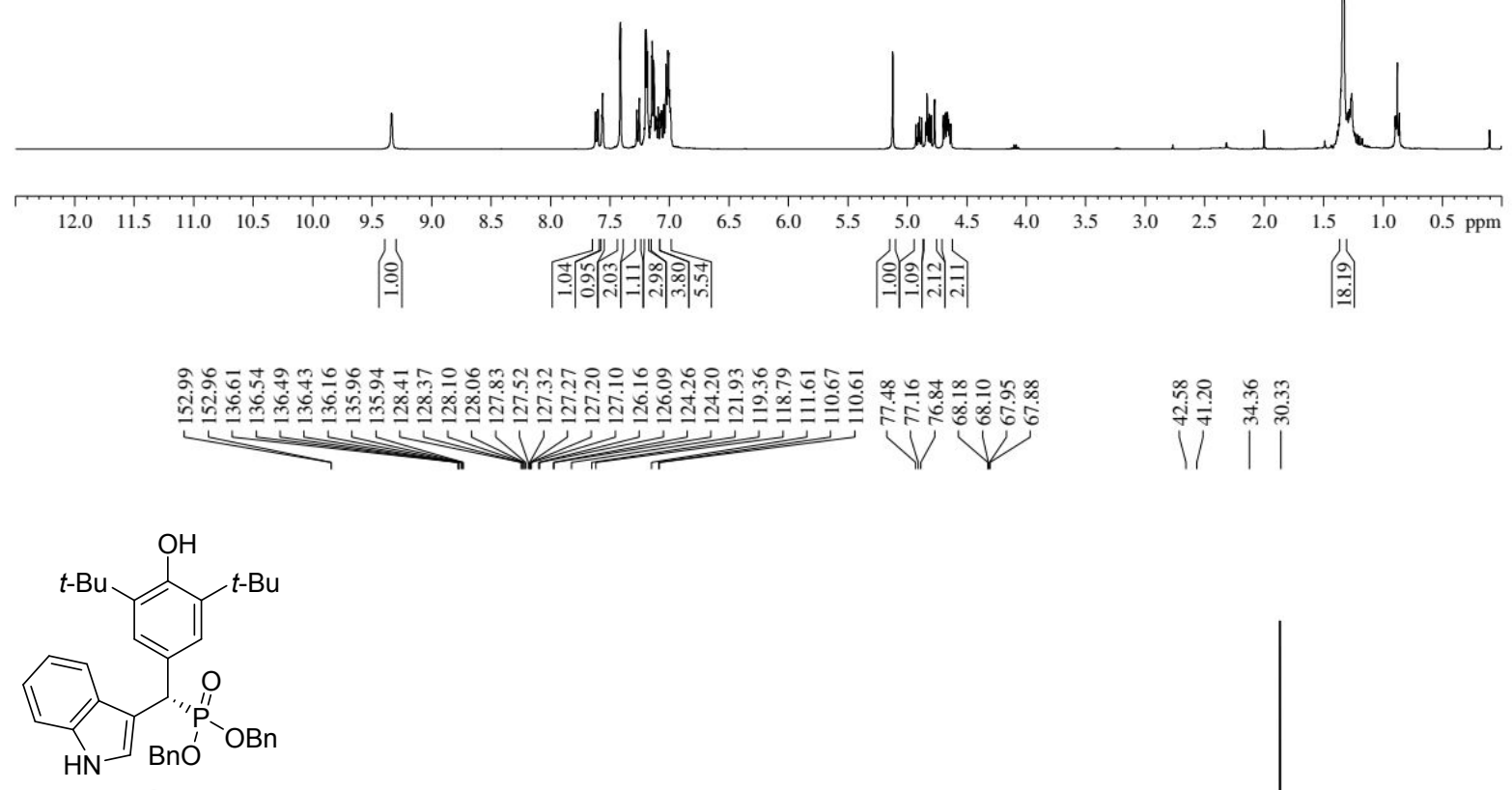

$3 \mathrm{~h}$

${ }^{13} \mathrm{C}\left\{{ }^{1} \mathrm{H}\right\}-\mathrm{NMR}\left(100 \mathrm{MHz}, \mathrm{CDCl}_{3}\right)$
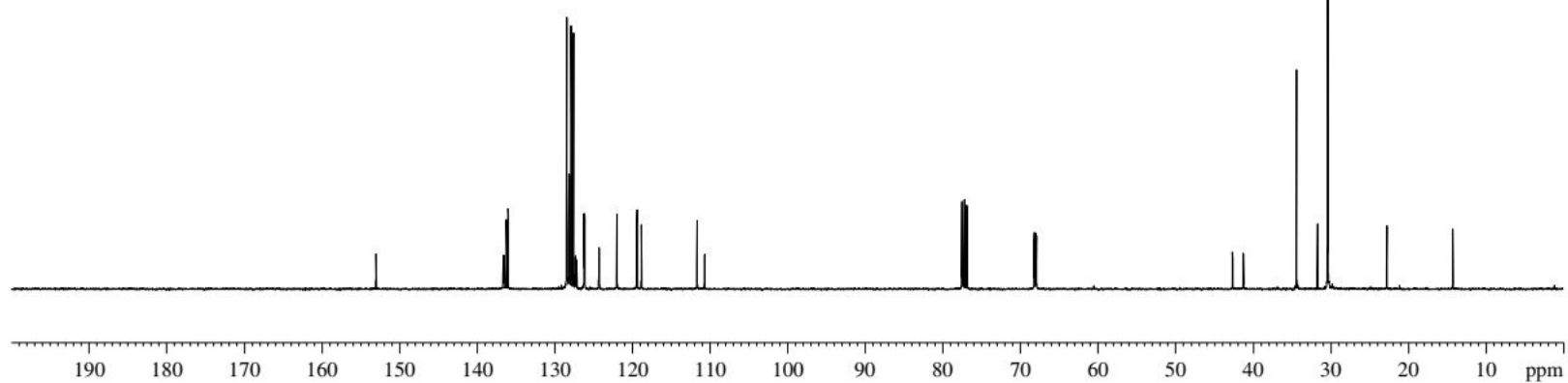

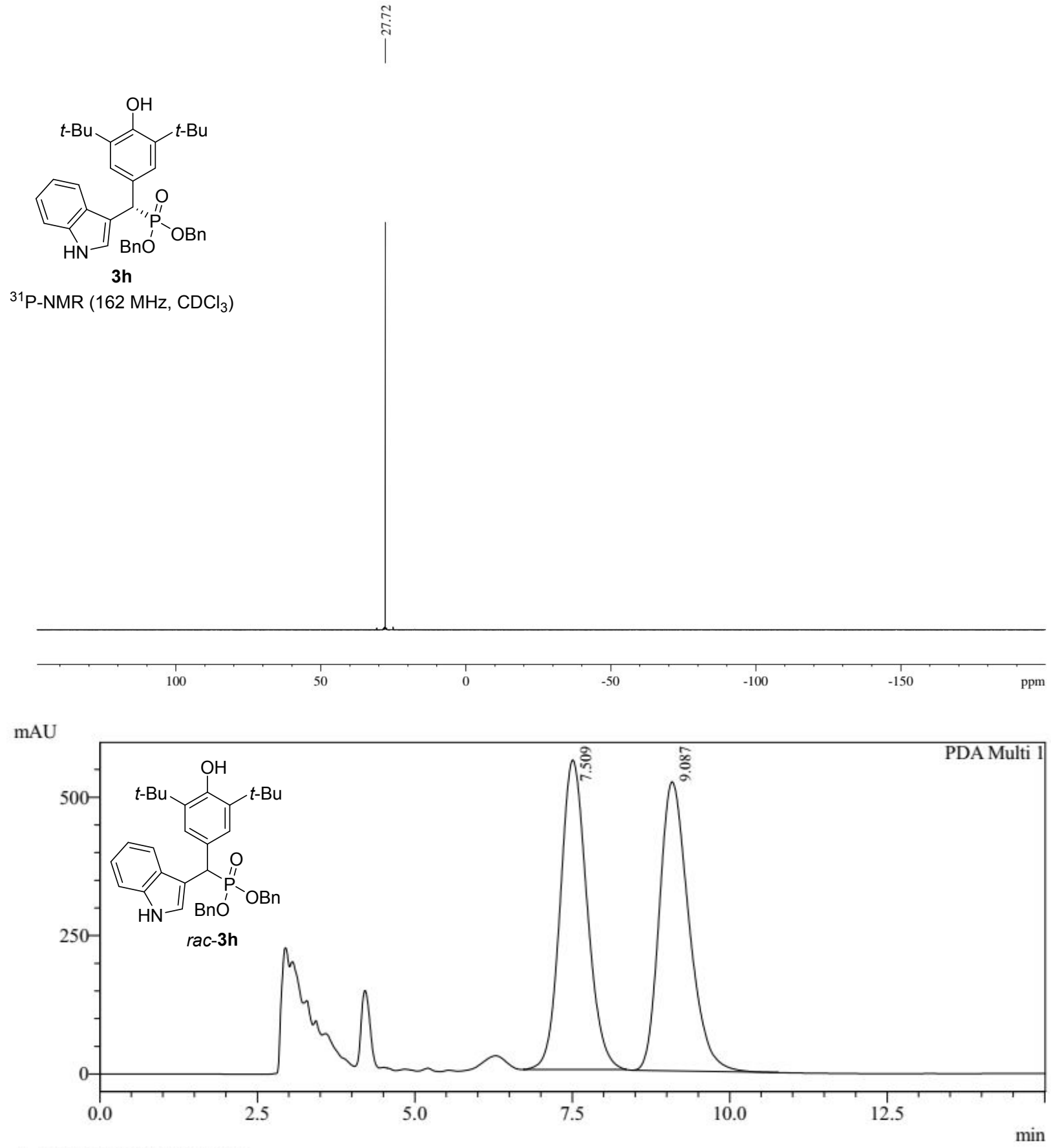

1 PDA Multi $1 / 224 \mathrm{~nm}, 4 \mathrm{~nm}$

\begin{tabular}{|c|c|c|c|c|}
\hline \multicolumn{5}{|c|}{ PDAChl 224nm } \\
\hline Peak\# & Ret. Time & Area & Height & Area $\%$ \\
\hline 1 & 7.509 & 16580405 & 559126 & 49.352 \\
\hline 2 & 9.087 & 17016125 & 522272 & 50.648 \\
\hline Total & & 33596530 & 1081398 & 100.000 \\
\hline
\end{tabular}




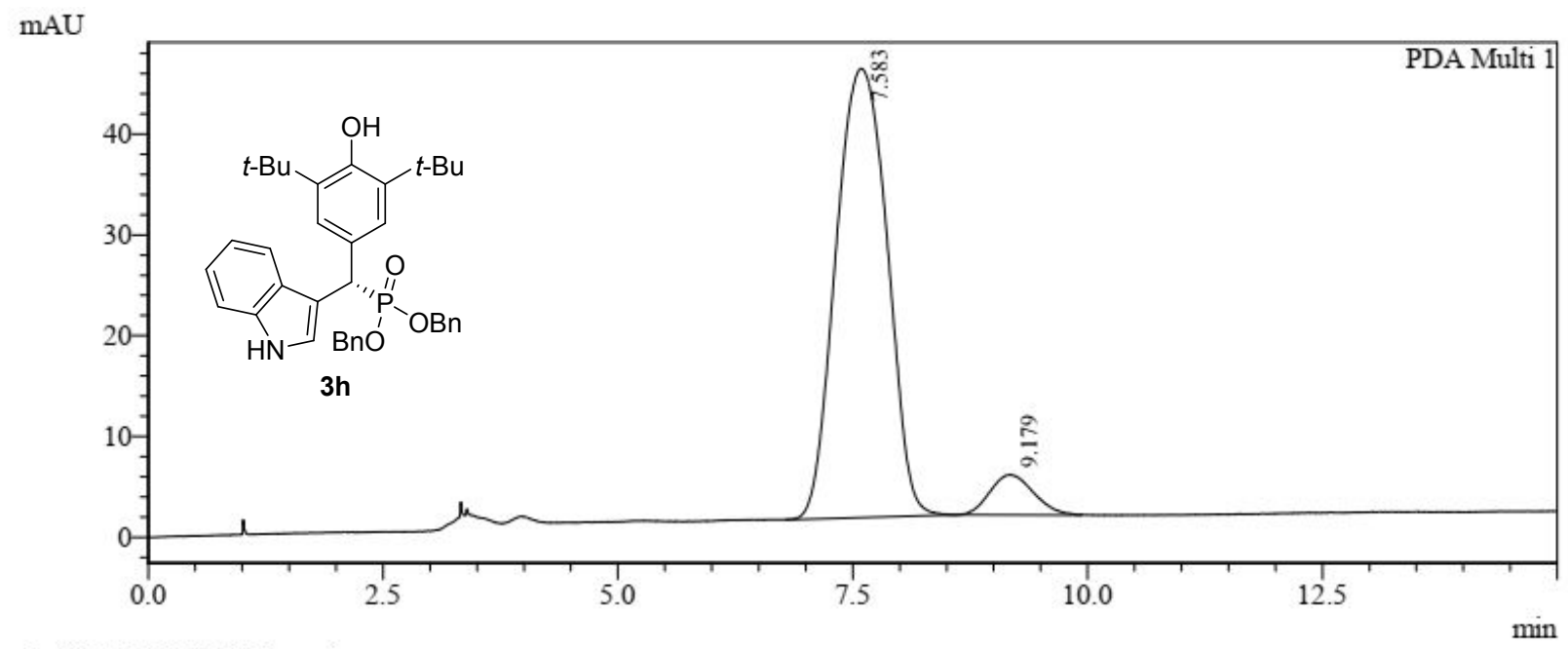

1 PDA Multi $1 / 224 \mathrm{~nm} 4 \mathrm{~nm}$

Ch1 224nm 4nm
\begin{tabular}{|r|r|r|r|r|}
\hline Peak\# & Ret. Time & \multicolumn{1}{|c|}{ Area } & Height & Area \% \\
\hline 1 & 7.583 & 1695415 & 44564 & 92.933 \\
\hline 2 & 9.179 & 128923 & 3998 & 7.067 \\
\hline Total & & 1824338 & 48562 & 100.000 \\
\hline
\end{tabular}

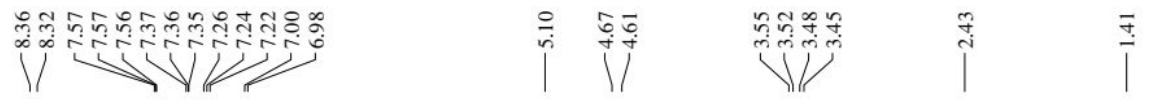
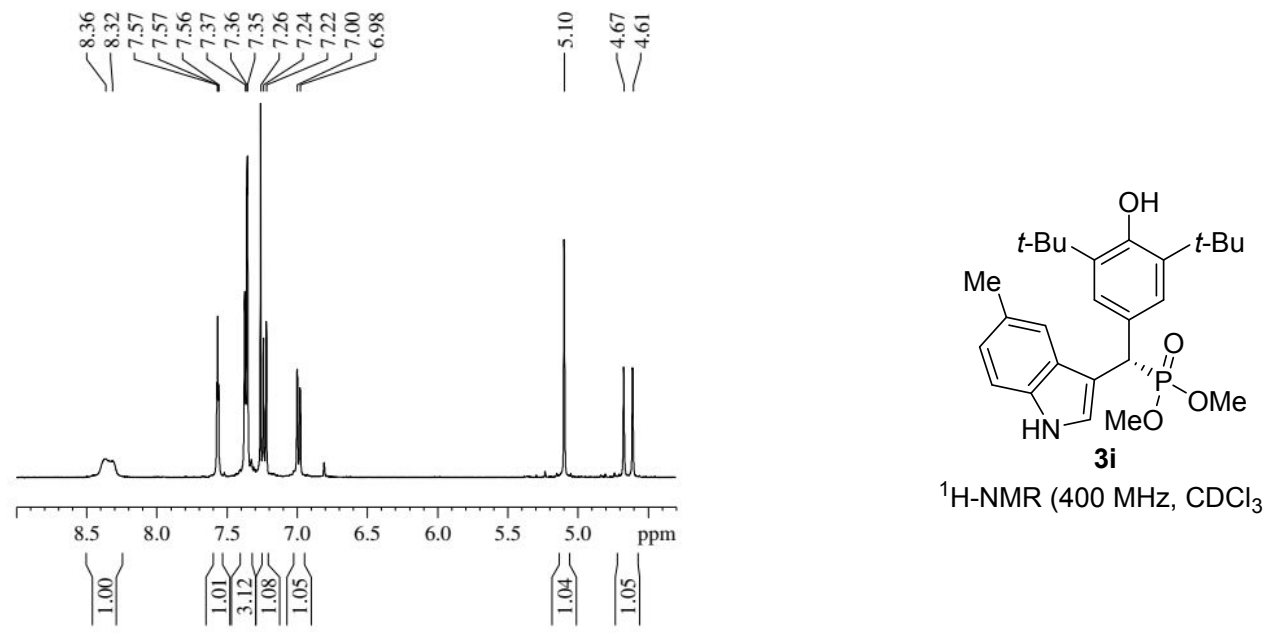

${ }^{1} \mathrm{H}-\mathrm{NMR}\left(400 \mathrm{MHz}, \mathrm{CDCl}_{3}\right)$

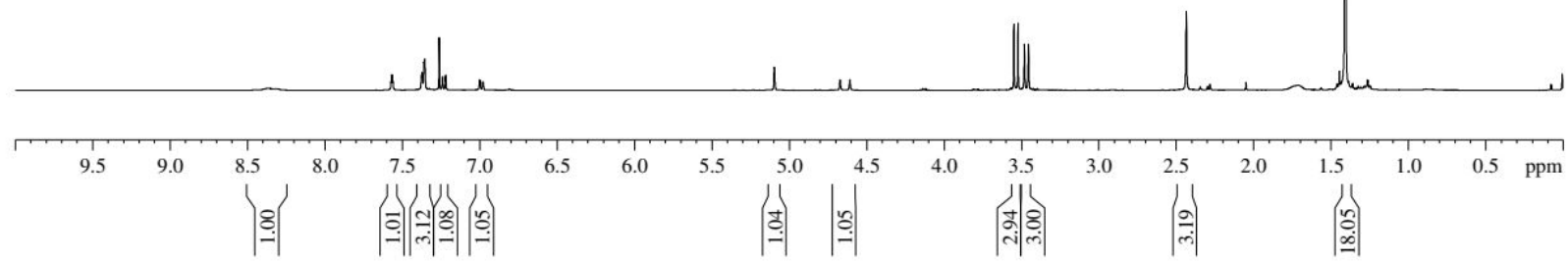


Arylated-Methyl Organophosphorus Compounds, Tripathi et al., SI-Part-B, Page S-44
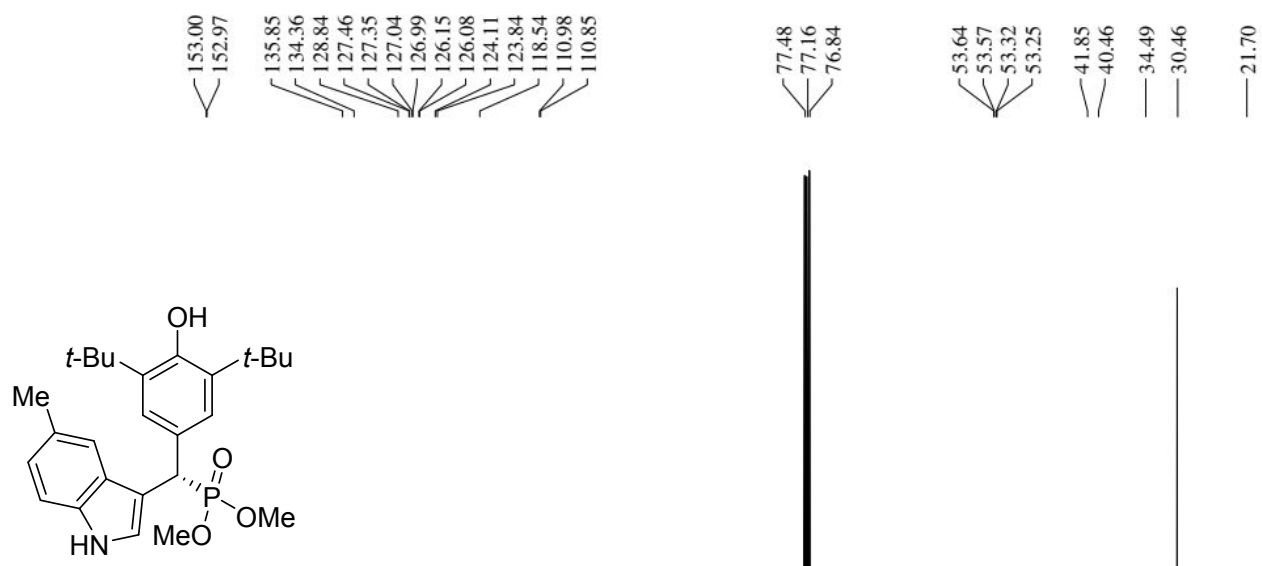

3i

${ }^{13} \mathrm{C}\left\{{ }^{1} \mathrm{H}\right\}-N M R\left(100 \mathrm{MHz}, \mathrm{CDCl}_{3}\right)$
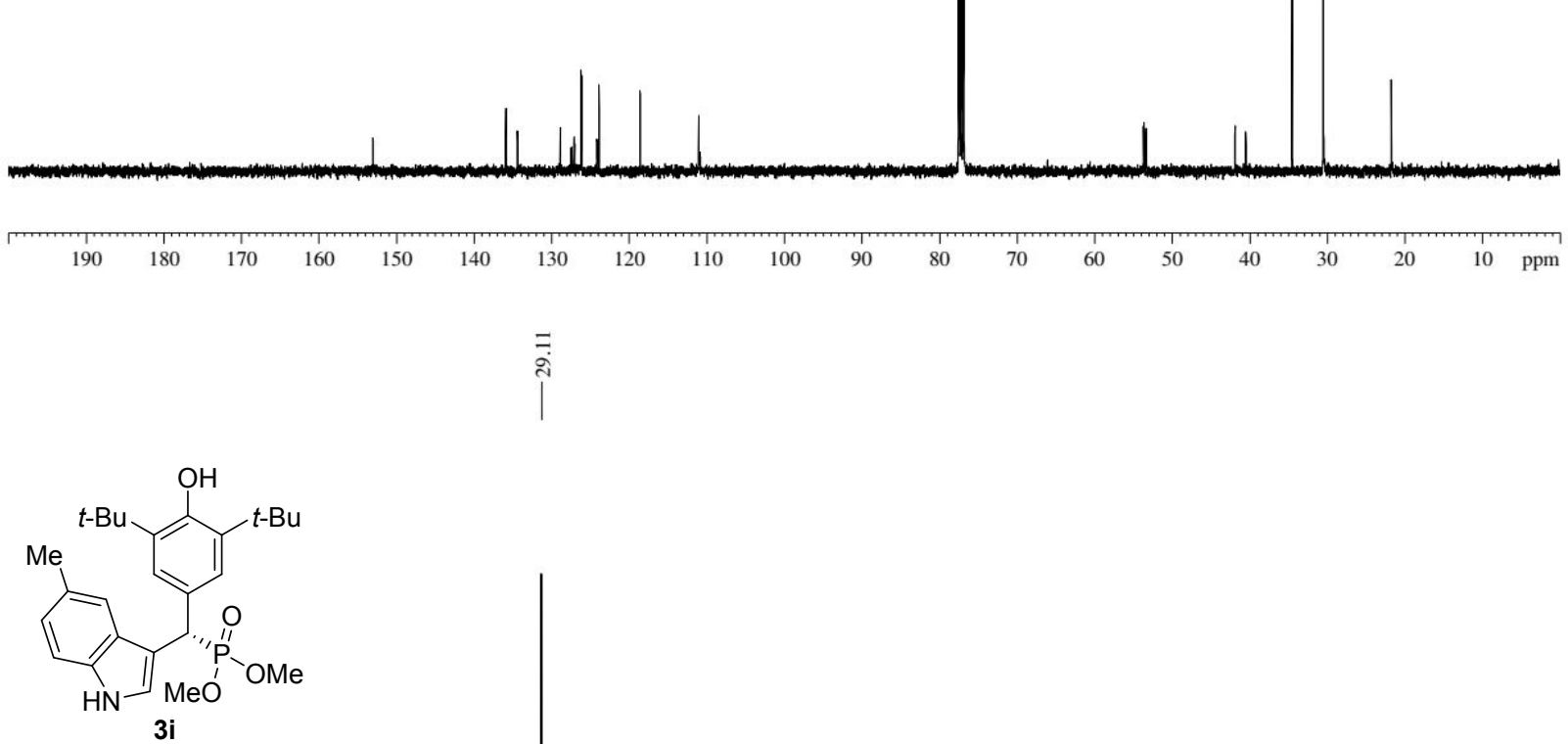

${ }^{31} \mathrm{P}-\mathrm{NMR}\left(162 \mathrm{MHz}, \mathrm{CDCl}_{3}\right.$ )

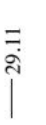


mAU

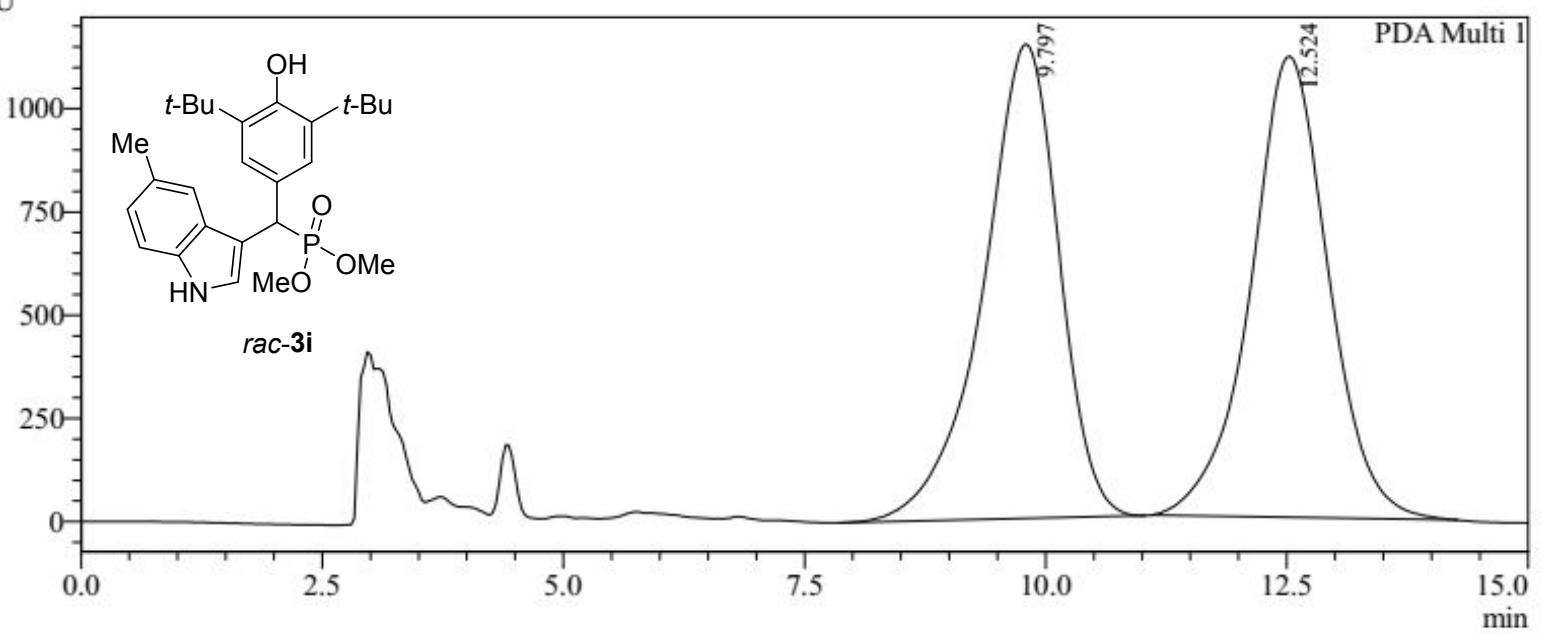

1 PDA Multi $1 / 224 \mathrm{~nm}, 4 \mathrm{~nm}$

\begin{tabular}{|c|c|c|c|c|}
\hline \multicolumn{5}{|c|}{ PDA Chl $224 \mathrm{~nm}$} \\
\hline Peak\# & Ret. Time & Area & Height & Area $\%$ \\
\hline 1 & 9.797 & 62827861 & 1148607 & 50.075 \\
\hline 2 & 12.524 & 62640328 & 1116516 & 49.925 \\
\hline Total & & 125468189 & 2265123 & 100.000 \\
\hline
\end{tabular}

mAU

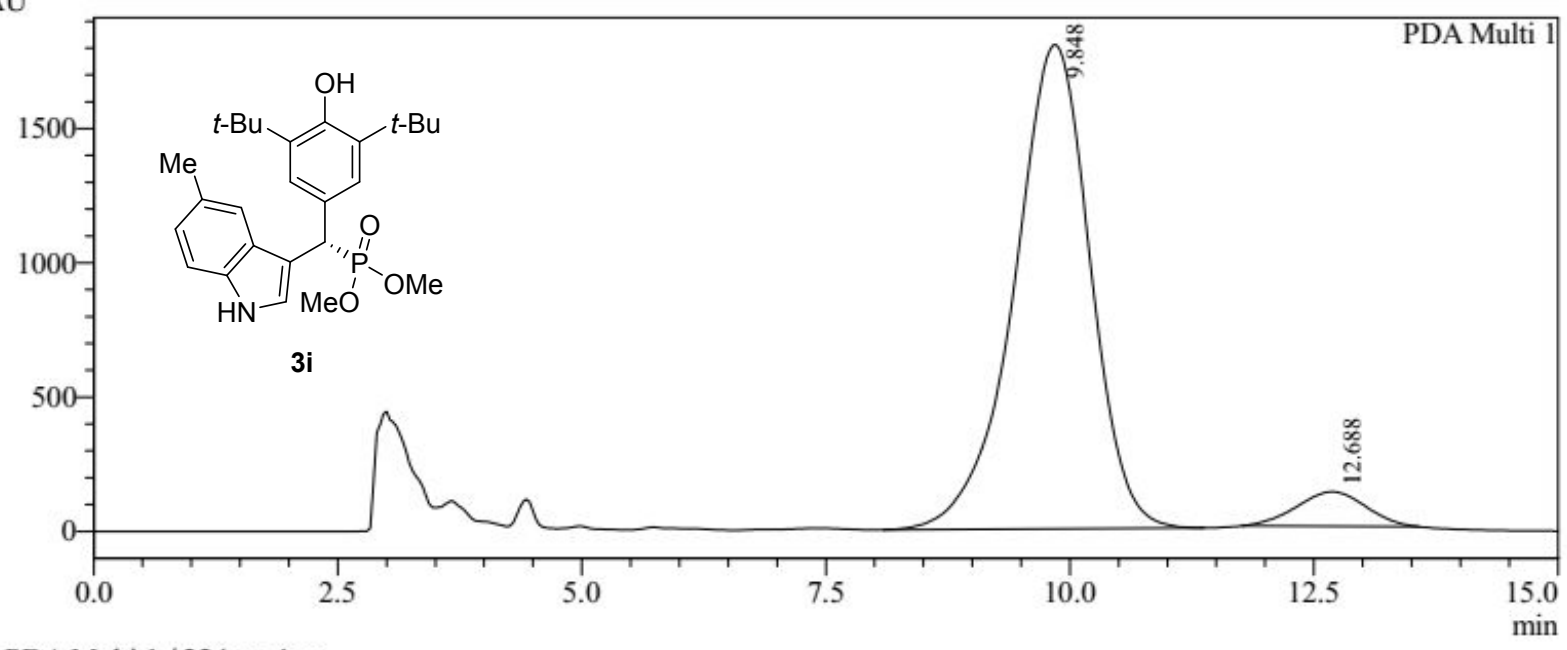

1 PDA Multi $1 / 224 \mathrm{~nm}, 4 \mathrm{~nm}$

\begin{tabular}{|c|c|c|c|c|}
\hline \multicolumn{5}{|c|}{ PDAChl 224nm } \\
\hline Peak\# & Ret. Time & Area & Height & Area \% \\
\hline 1 & 9.848 & 97496301 & 1802298 & 93.900 \\
\hline 2 & 12.688 & 6333911 & 127970 & 6.100 \\
\hline Total & & 103830212 & 1930268 & 100.000 \\
\hline
\end{tabular}



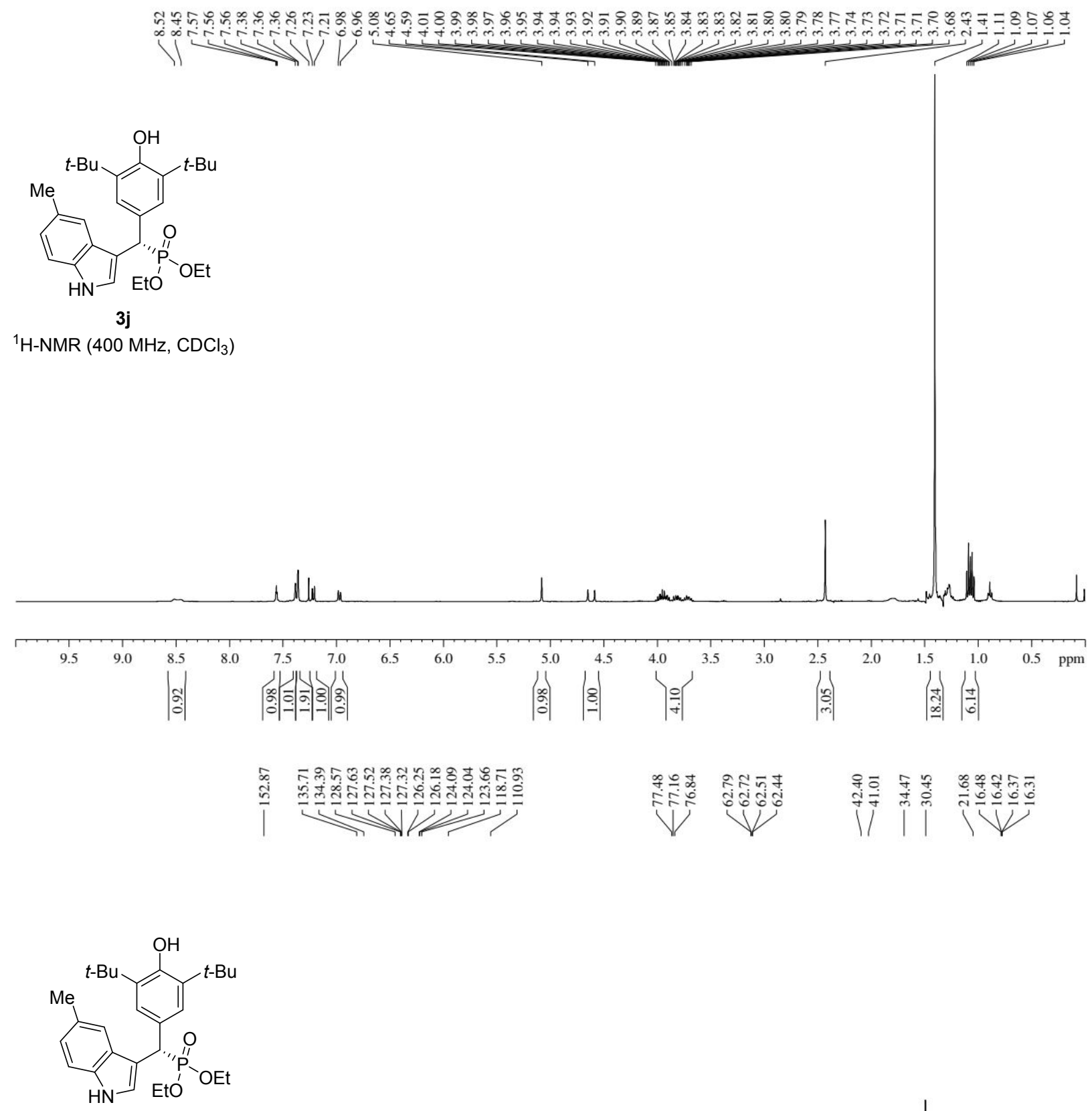

$3 \mathbf{j}$

${ }^{13} \mathrm{C}\left\{{ }^{1} \mathrm{H}\right\}$-NMR $\left(100 \mathrm{MHz}, \mathrm{CDCl}_{3}\right)$

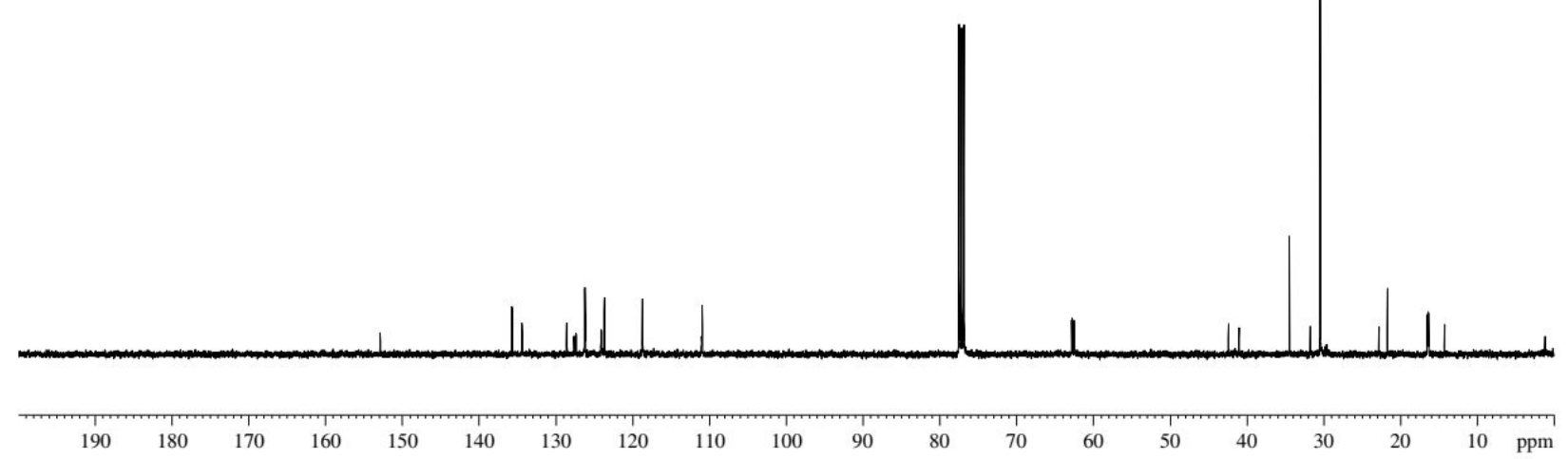




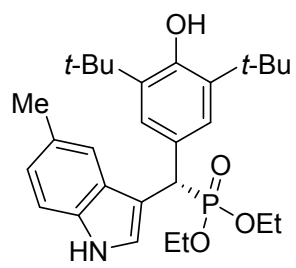

3j

${ }^{31} \mathrm{P}-\mathrm{NMR}\left(162 \mathrm{MHz}, \mathrm{CDCl}_{3}\right)$

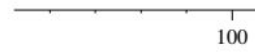

$00 \quad 50$

0

$-50$

$-100$

$-150$ ppm

mAU

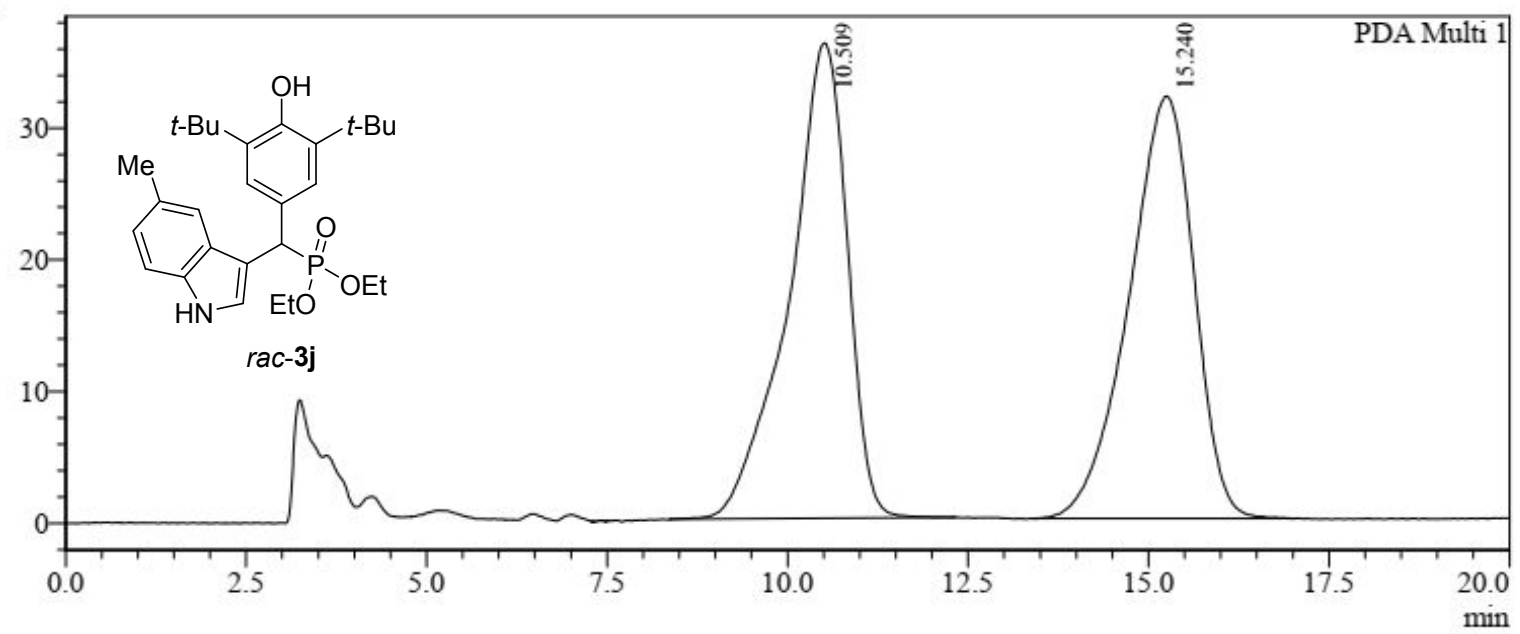

1 PDA Multi $1 / 230 \mathrm{~nm} 4 \mathrm{~nm}$

PeakTable

Ch1 230nm 4nm
\begin{tabular}{|r|r|r|r|r|}
\hline Peak\# & Ret. Time & Area & Height & \multicolumn{1}{|c|}{ Area \% } \\
\hline 1 & 10.509 & 2016736 & 36061 & 50.001 \\
\hline 2 & 15.240 & 2016689 & 32058 & 49.999 \\
\hline Total & & 4033426 & 68118 & 100.000 \\
\hline
\end{tabular}


mAU

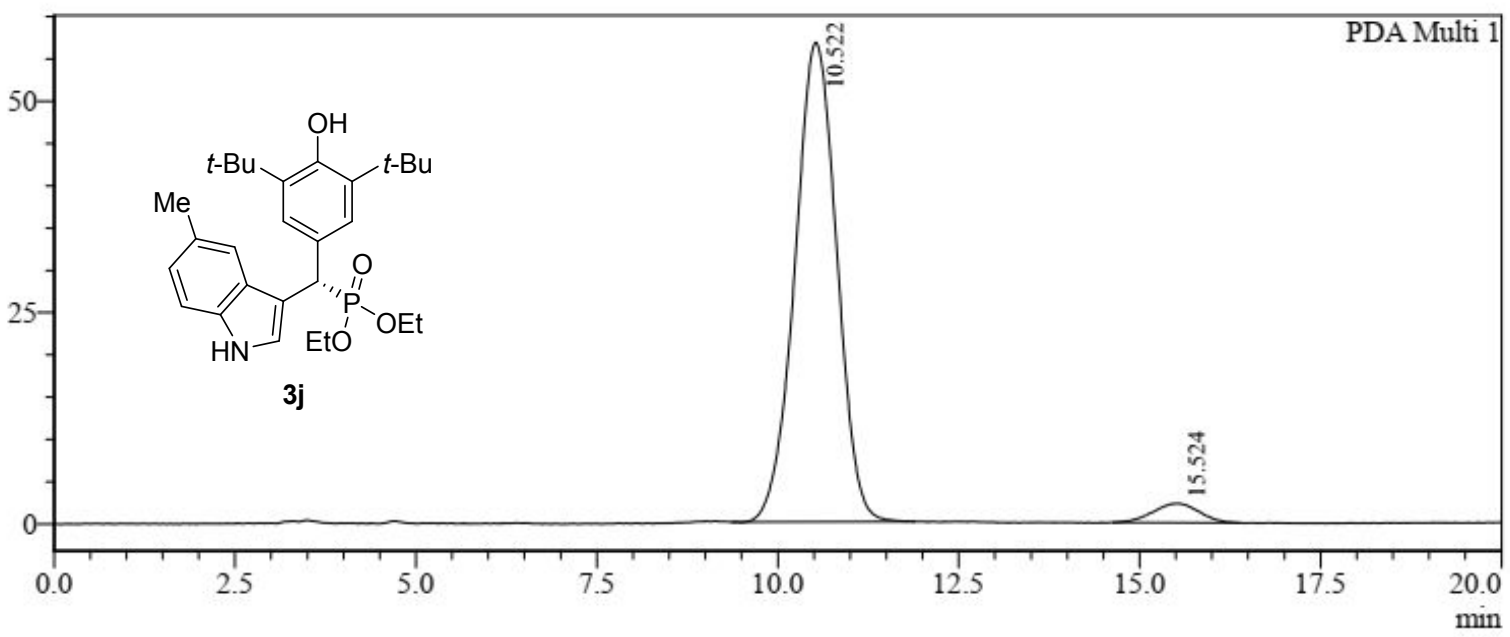

1 PDA Multi $1 / 230 \mathrm{~nm} 4 \mathrm{~nm}$

Ch1 230nm 4nm
\begin{tabular}{|r|r|r|r|r|}
\hline \multicolumn{5}{|c|}{ PeakTable } \\
\hline 1 & Reak. Time & \multicolumn{1}{c|}{ Area } & \multicolumn{1}{c|}{ Height } & \multicolumn{1}{c|}{ Area \% } \\
\hline 2 & 10.522 & 2281555 & 56678 & 95.835 \\
\hline Total & 15.524 & 99146 & 2254 & 4.165 \\
\hline & & 2380701 & 58932 & 100.000 \\
\hline
\end{tabular}
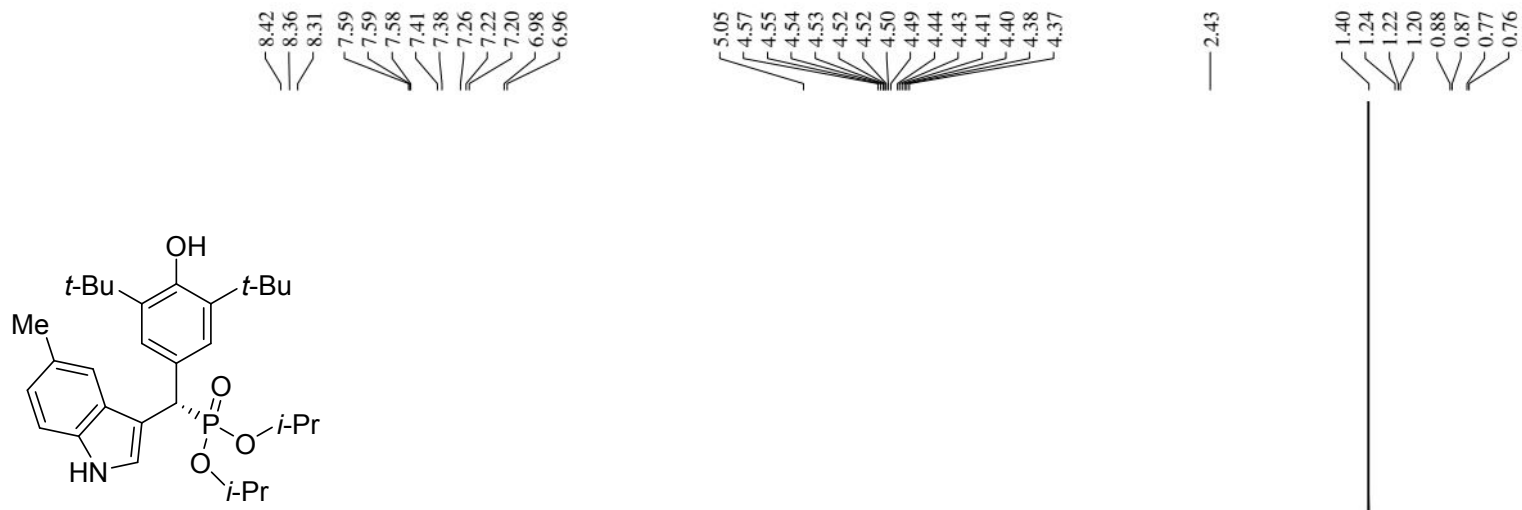

$3 \mathbf{k}$

${ }^{1} \mathrm{H}-\mathrm{NMR}\left(400 \mathrm{MHz}, \mathrm{CDCl}_{3}\right.$ )

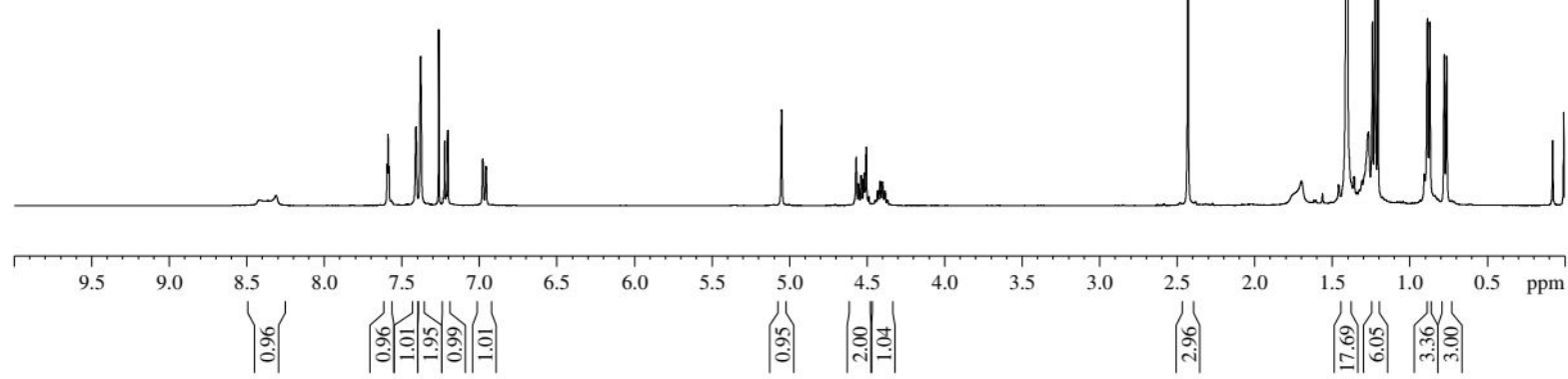



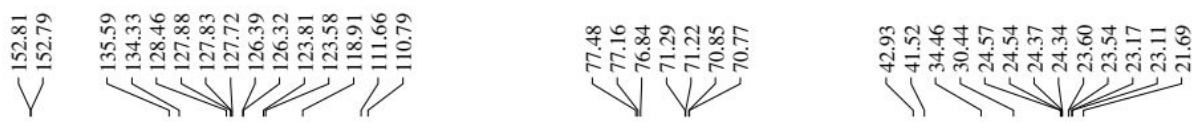

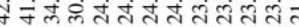

$1)$

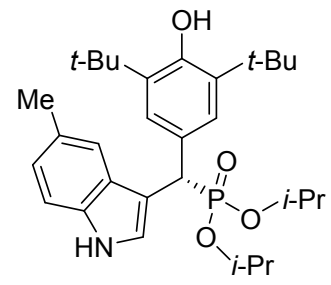

3k

${ }^{13} \mathrm{C}\left\{{ }^{1} \mathrm{H}\right\}-\mathrm{NMR}\left(100 \mathrm{MHz}, \mathrm{CDCl}_{3}\right)$

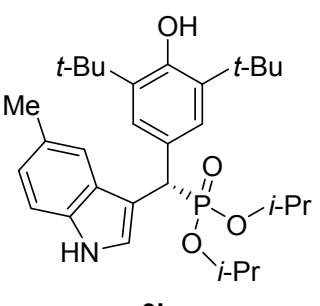

3k

${ }^{31} \mathrm{P}-\mathrm{NMR}\left(162 \mathrm{MHz}, \mathrm{CDCl}_{3}\right)$

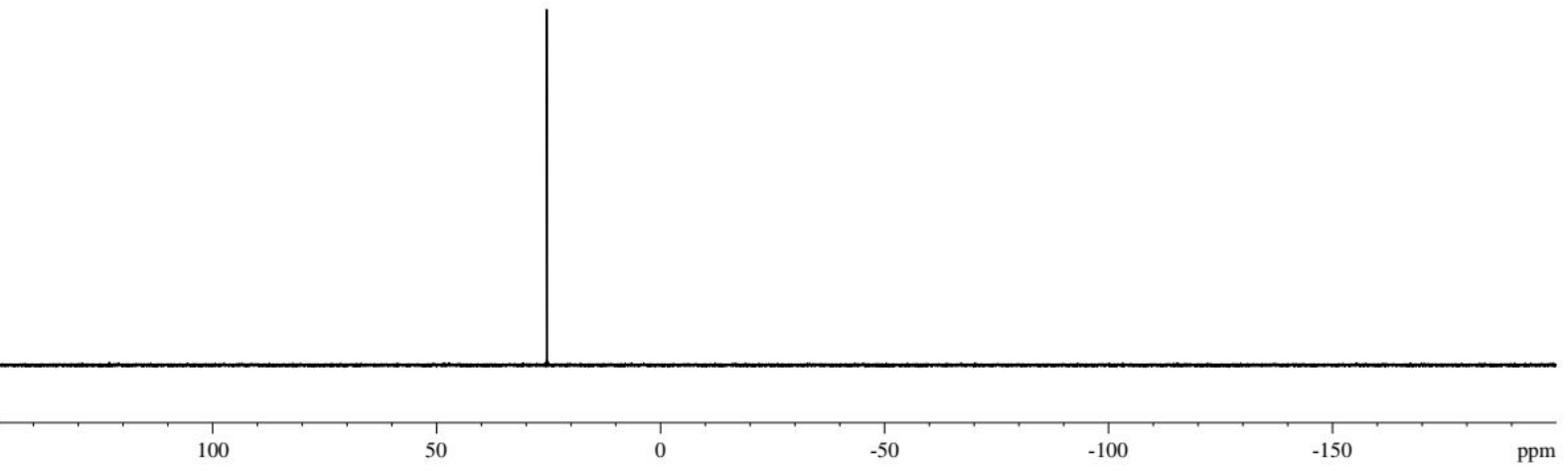


mAU

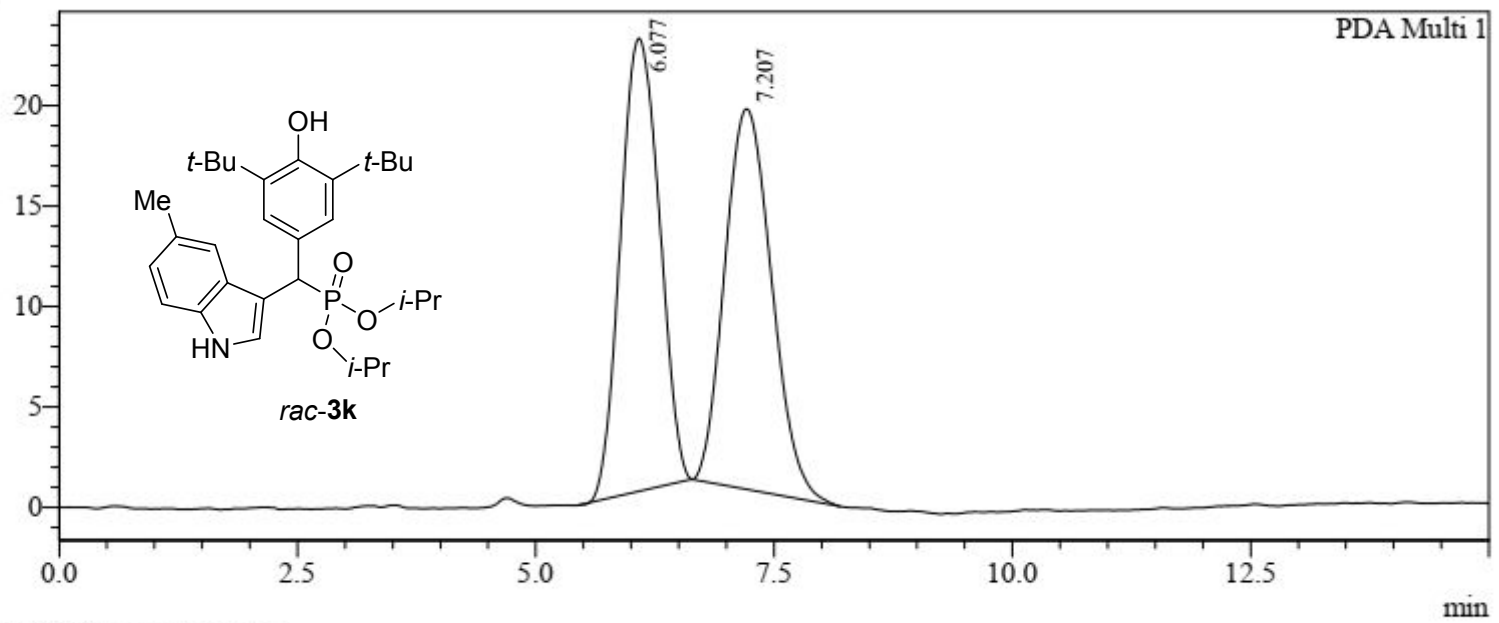

1 PDA Multi $1 / 224 \mathrm{~nm} 4 \mathrm{~nm}$

PeakTable

Ch1 $224 \mathrm{~nm} 4 \mathrm{~nm}$
\begin{tabular}{|r|r|r|r|r|}
\hline Peak\# & Ret. Time & \multicolumn{1}{|c|}{ Area } & Height & Area $\%$ \\
\hline 1 & 6.077 & 641891 & 22560 & 49.749 \\
\hline 2 & 7.207 & 648365 & 18937 & 50.251 \\
\hline Total & & 1290257 & 41497 & 100.000 \\
\hline
\end{tabular}

mAU

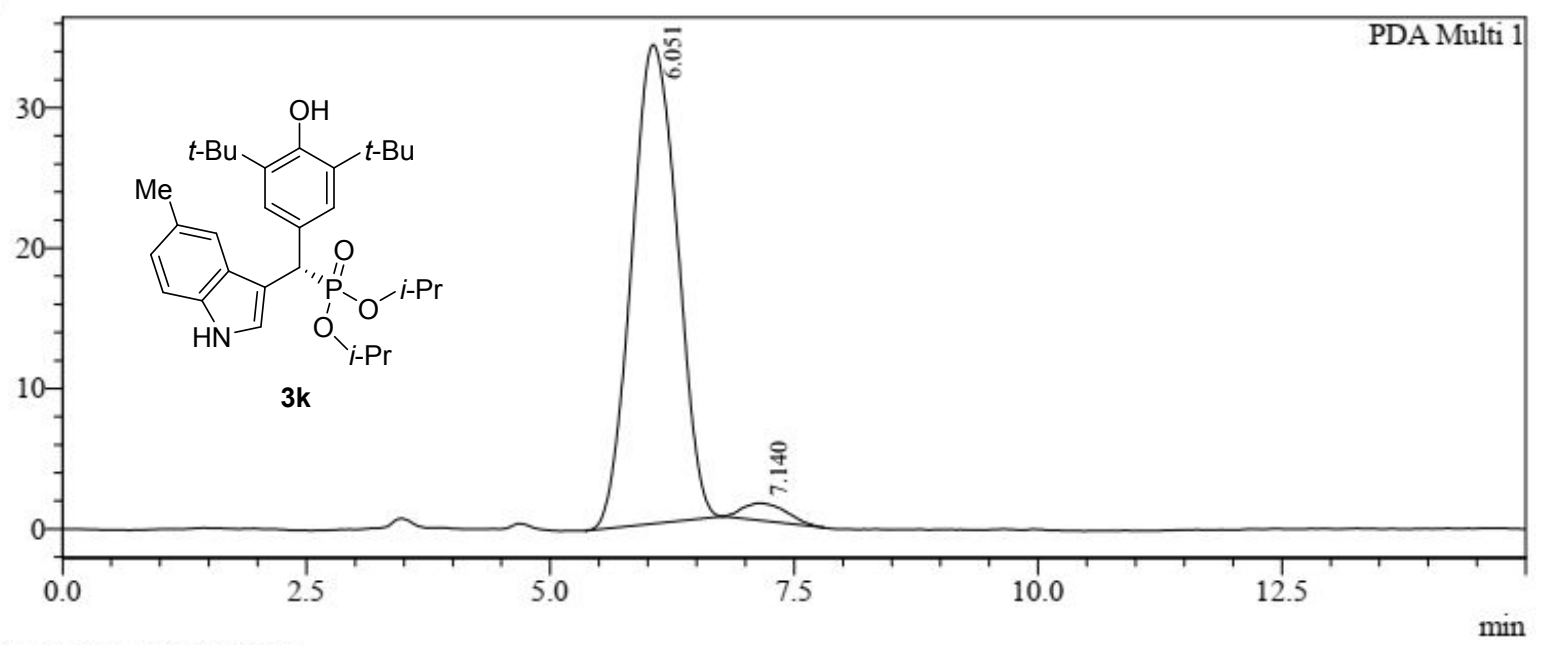

1 PDA Multi $1 / 224 \mathrm{~nm} 4 \mathrm{~nm}$

PeakTable

Ch1 224nm 4nm
\begin{tabular}{|r|r|r|r|r|}
\hline Peak\# & Ret. Time & \multicolumn{1}{|c|}{ Area } & Height & \multicolumn{1}{|c|}{ Area \% } \\
\hline 1 & 6.051 & 1109498 & 34114 & 96.564 \\
\hline 2 & 7.140 & 39481 & 1228 & 3.436 \\
\hline Total & & 1148979 & 35342 & 100.000 \\
\hline
\end{tabular}




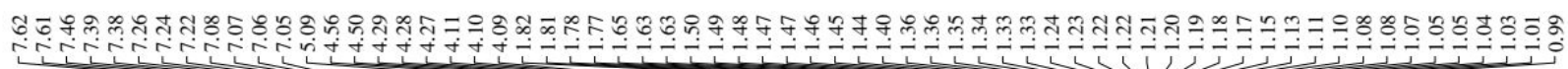<smiles>CCOP(=O)(OC[14CH3])C(c1cc(C(C)(C)C)c(O)c(C(C)(C)C)c1)c1c[nH]c2ccc(Cl)cc12</smiles>

3!

${ }^{1} \mathrm{H}-\mathrm{NMR}\left(400 \mathrm{MHz}, \mathrm{CDCl}_{3}\right)$

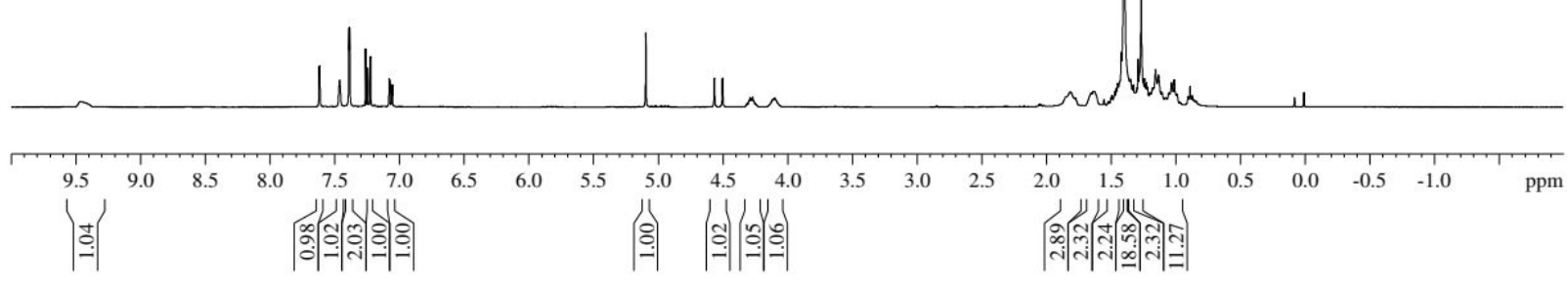

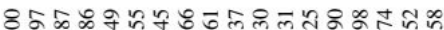

V

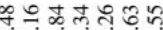

Finosin

N

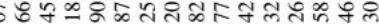

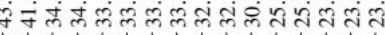

年<smiles>[R]COP(=O)(OC[R])C(c1cc(C(C)(C)C)c(O)c(C(C)(C)C)c1)c1c[nH]c2ccc(Cl)cc12</smiles>

31

${ }^{13} \mathrm{C}\left\{{ }^{1} \mathrm{H}\right\}-N M R\left(100 \mathrm{MHz}, \mathrm{CDCl}_{3}\right)$

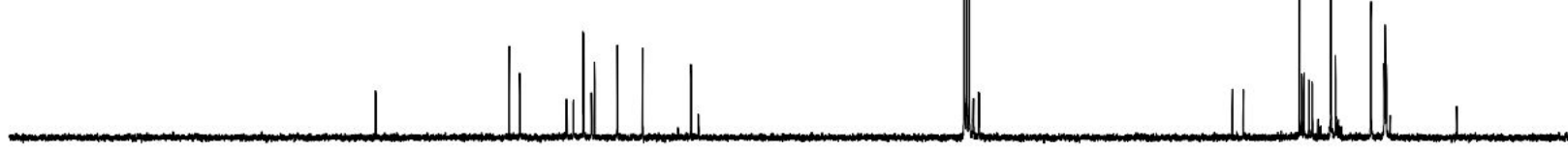




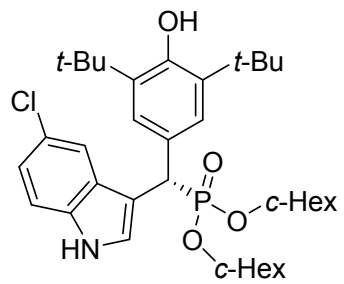

31

${ }^{31} \mathrm{P}-\mathrm{NMR}\left(162 \mathrm{MHz}, \mathrm{CDCl}_{3}\right.$ )

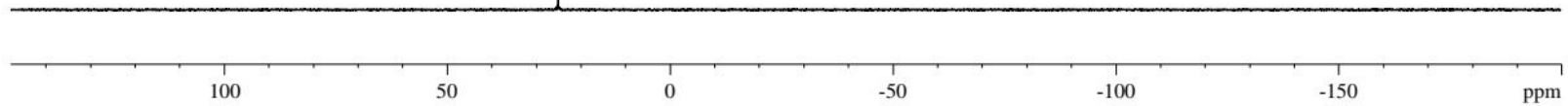

mAU

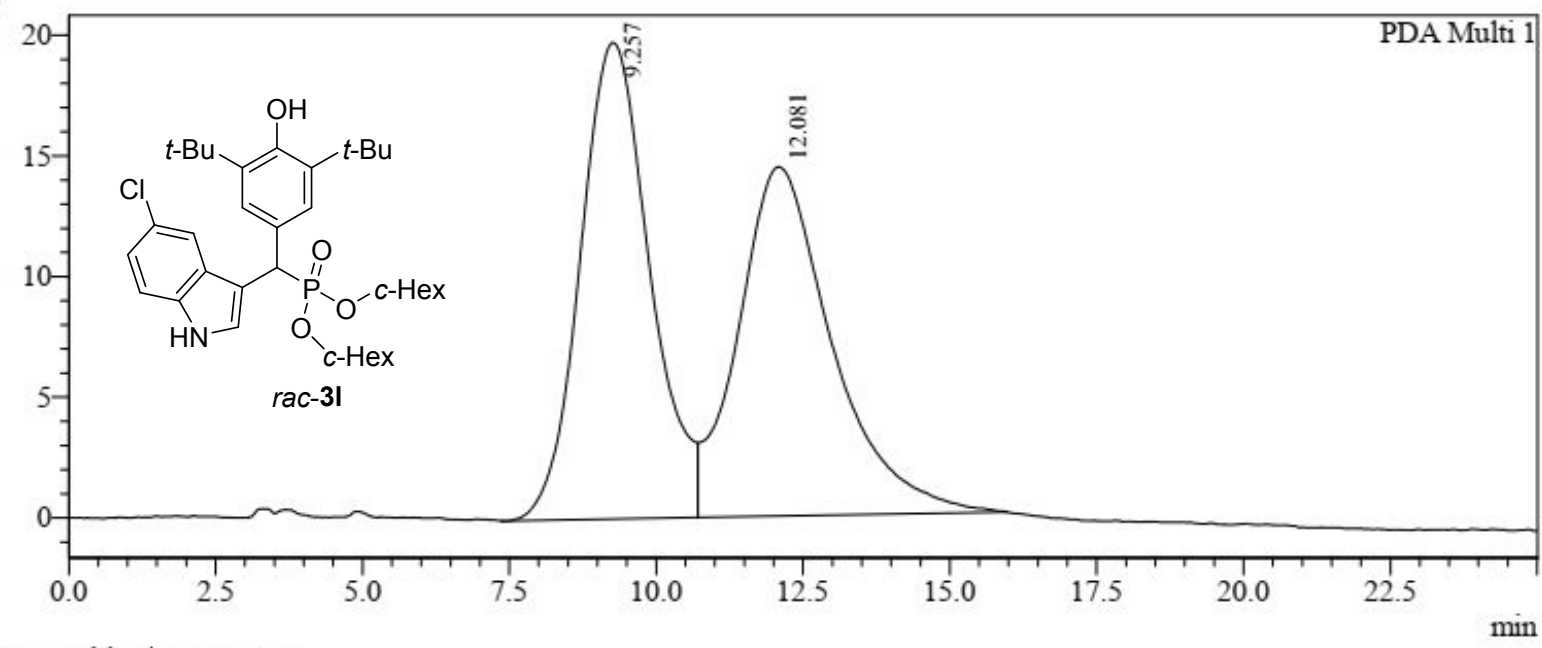

1 PDA Multi $1 / 230 \mathrm{~nm} 4 \mathrm{~nm}$

PeakTable

Ch1 230nm 4nm
\begin{tabular}{|r|r|r|r|r|}
\hline Peak\# & Ret. Time & Area & Height & Area $\%$ \\
\hline 1 & 9.257 & 1624157 & 19737 & 50.266 \\
\hline 2 & 12.081 & 1606939 & 14474 & 49.734 \\
\hline Total & & 3231096 & 34211 & 100.000 \\
\hline
\end{tabular}


mAU

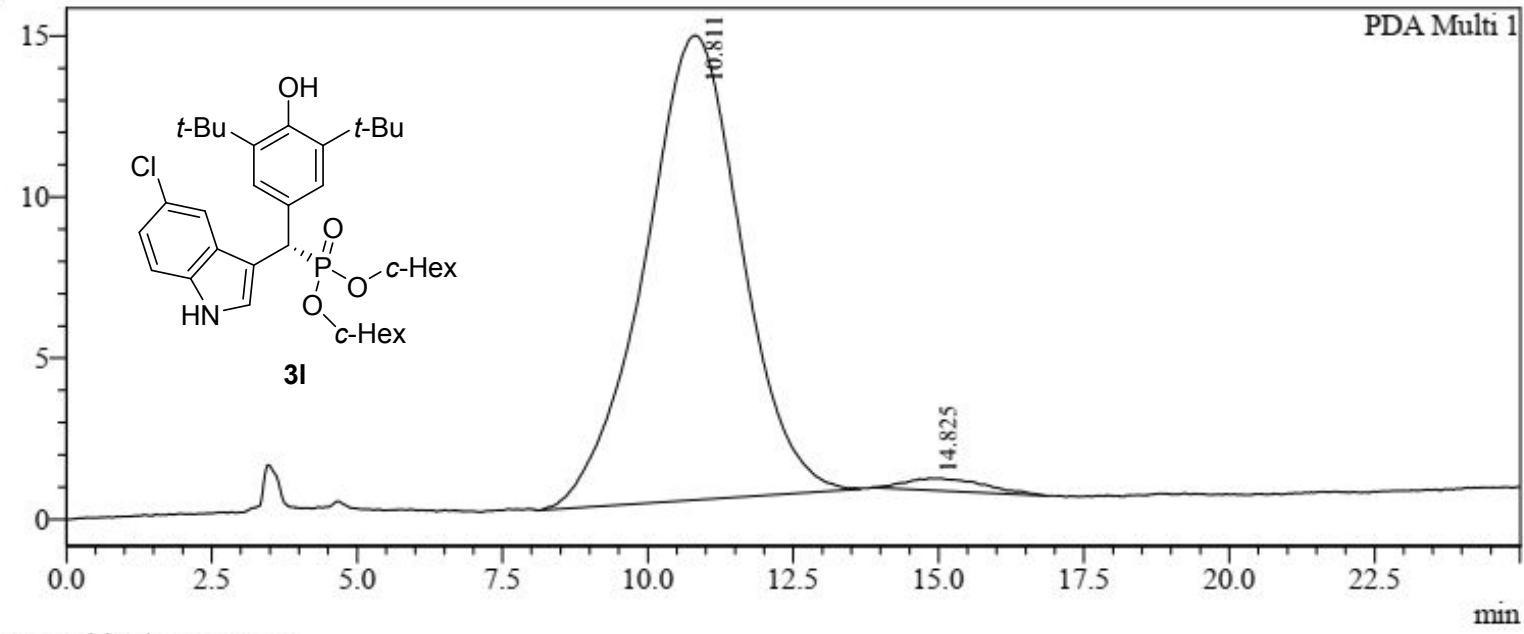

1 PDA Multi $1 / 230 \mathrm{~nm} 4 \mathrm{~nm}$

Ch1 230nm 4nm
\begin{tabular}{|r|r|r|r|r|}
\hline \multicolumn{1}{|c|}{ PeakTable } \\
\hline 1 & Ret. Time & Area & \multicolumn{1}{c|}{ Height } & Area $\%$ \\
\hline 2 & 10.811 & 1667464 & 14416 & 97.824 \\
\hline Total & 14.825 & 37089 & 376 & 2.176 \\
\hline & & 1704554 & 14791 & 100.000 \\
\hline
\end{tabular}

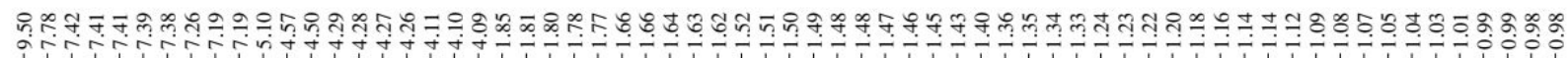
(1)<smiles>[R]COP(=O)(OC[Ga])C(c1cc(C(C)(C)C)c(O)c(C(C)(C)C)c1)c1c[nH]c2ccc(Br)cc12</smiles>

${ }^{1} \mathrm{H}-\mathrm{NMR}\left(400 \mathrm{MHz}, \mathrm{CDCl}_{3}\right)$

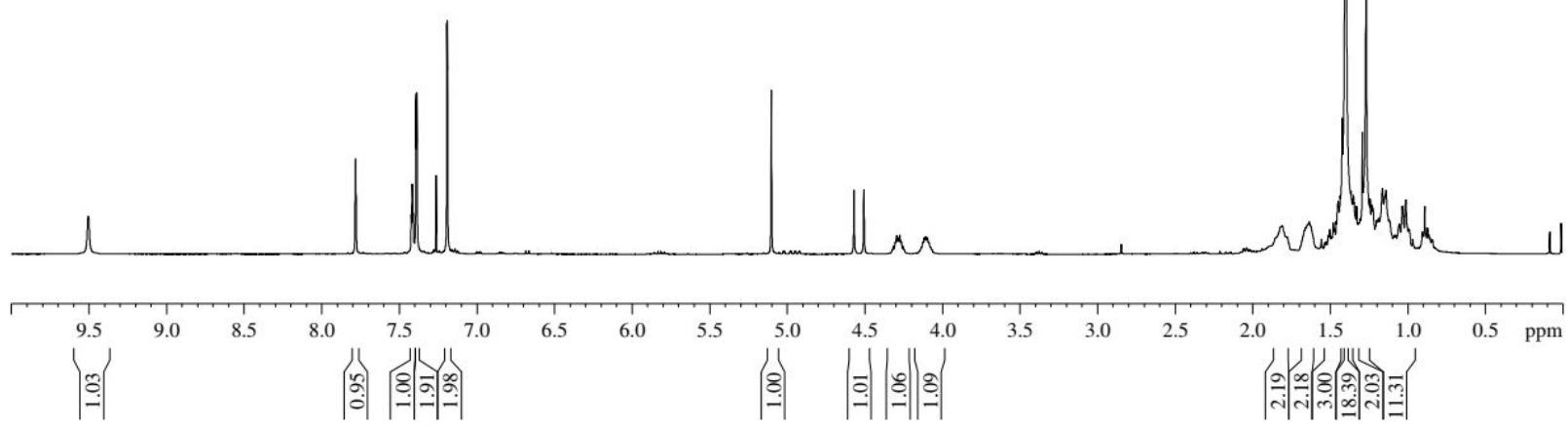



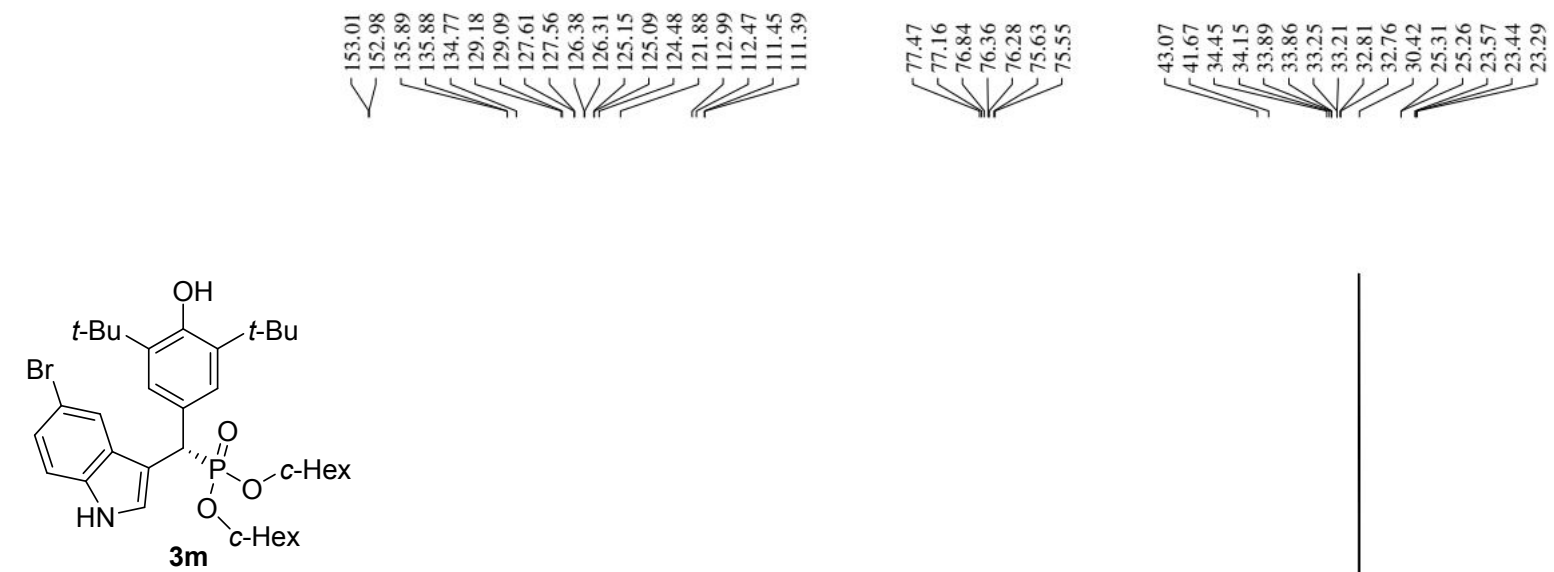

${ }^{13} \mathrm{C}\left\{{ }^{1} \mathrm{H}\right\}-\mathrm{NMR}\left(100 \mathrm{MHz}, \mathrm{CDCl}_{3}\right)$
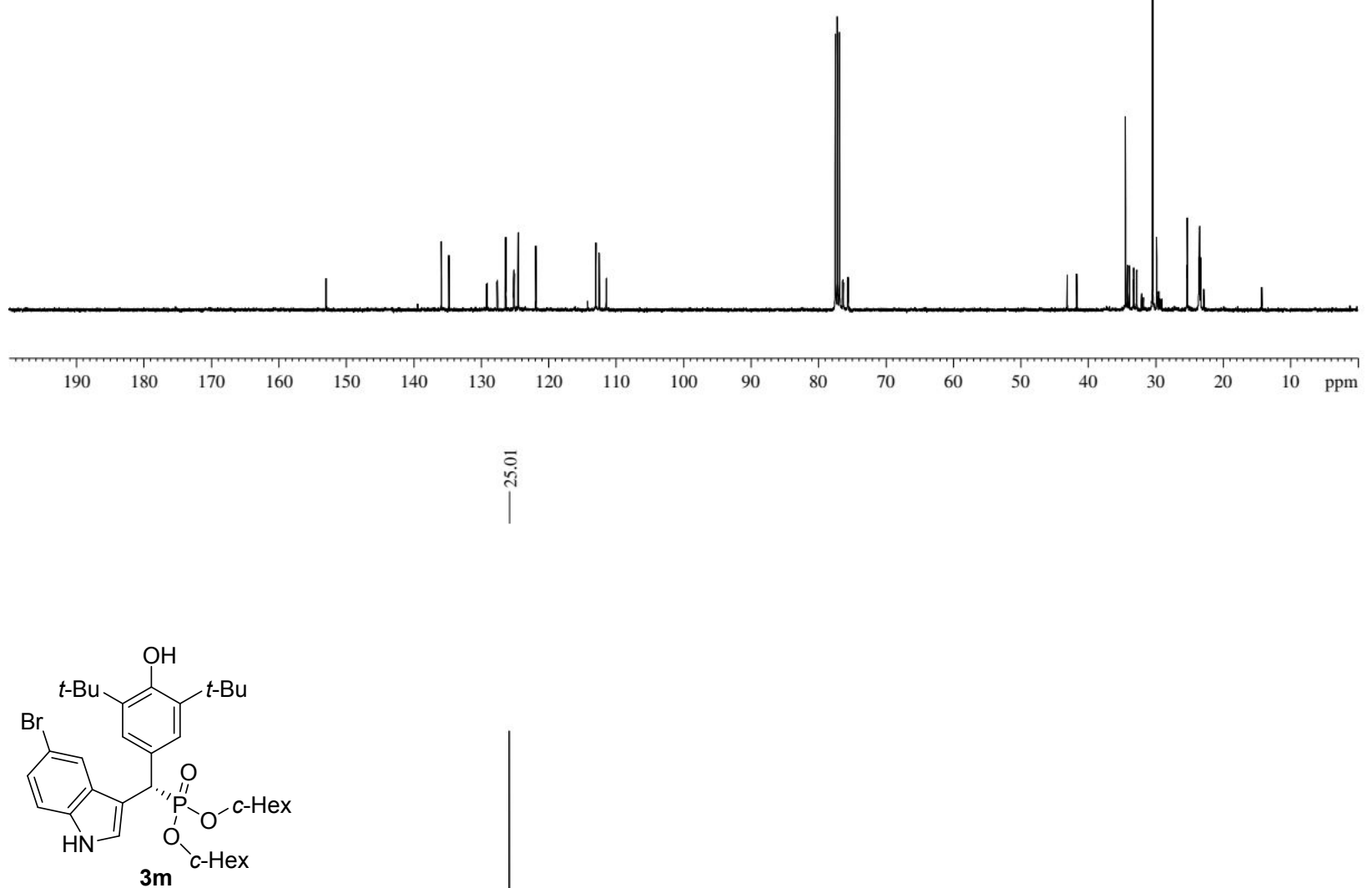

${ }^{31} \mathrm{P}-\mathrm{NMR}\left(162 \mathrm{MHz}, \mathrm{CDCl}_{3}\right.$ )

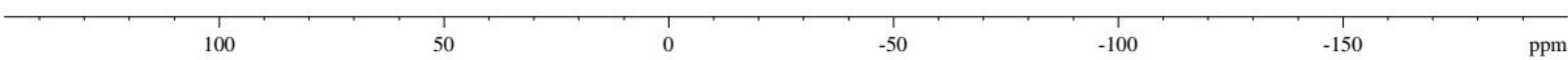


mAU

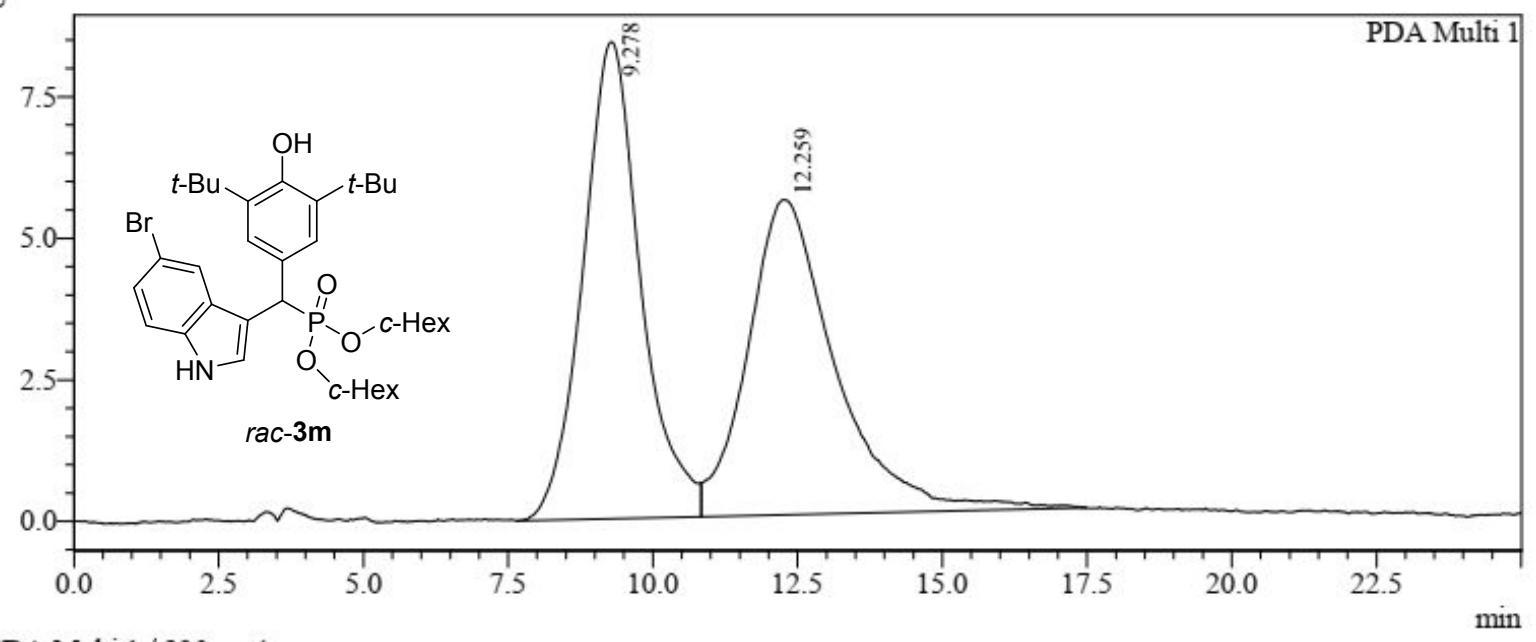

1 PDA Multi $1 / 230 \mathrm{~nm} 4 \mathrm{~nm}$

\begin{tabular}{|c|c|c|c|c|}
\hline \multicolumn{5}{|c|}{$\mathrm{Chl} 230 \mathrm{~nm} 4 \mathrm{~nm}$} \\
\hline Peak\# & Ret. Time & Area & Height & Area $\%$ \\
\hline 1 & 9.278 & 585878 & 8416 & 49.972 \\
\hline 2 & 12.259 & 586531 & 5562 & 50.028 \\
\hline Total & & 1172410 & 13978 & 100.000 \\
\hline
\end{tabular}

mAU

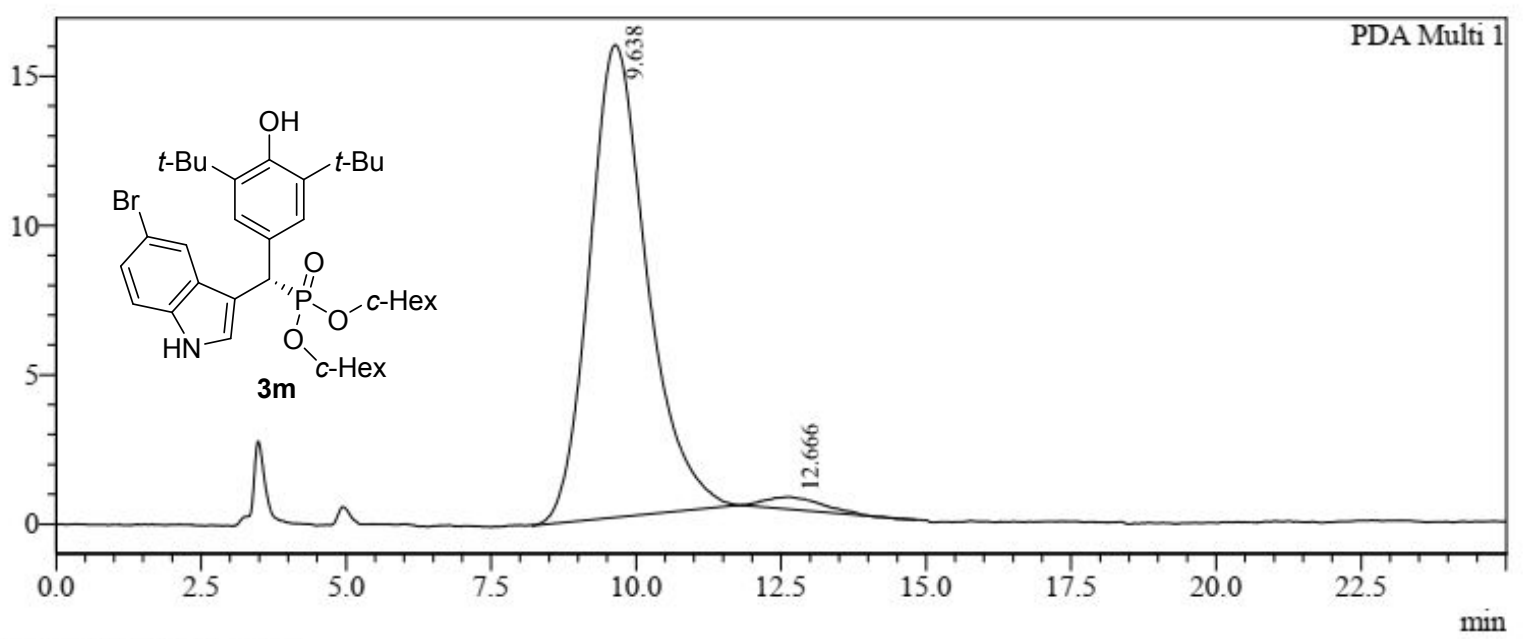

1 PDA Multi $1 / 230 \mathrm{~nm} 4 \mathrm{~nm}$

\begin{tabular}{|c|c|c|c|c|}
\hline \multicolumn{5}{|c|}{ Ch1 230nm 4nm } \\
\hline Peak\# & Ret. Time & Area & Height & Area \% \\
\hline 1 & 9.638 & 1094858 & 15815 & 97.513 \\
\hline 2 & 12.666 & 27924 & 416 & 2.487 \\
\hline Total & & 1122782 & 16232 & 100.000 \\
\hline
\end{tabular}




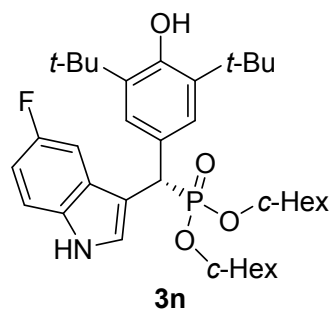

${ }^{1} \mathrm{H}-\mathrm{NMR}\left(400 \mathrm{MHz}, \mathrm{CDCl}_{3}\right)$
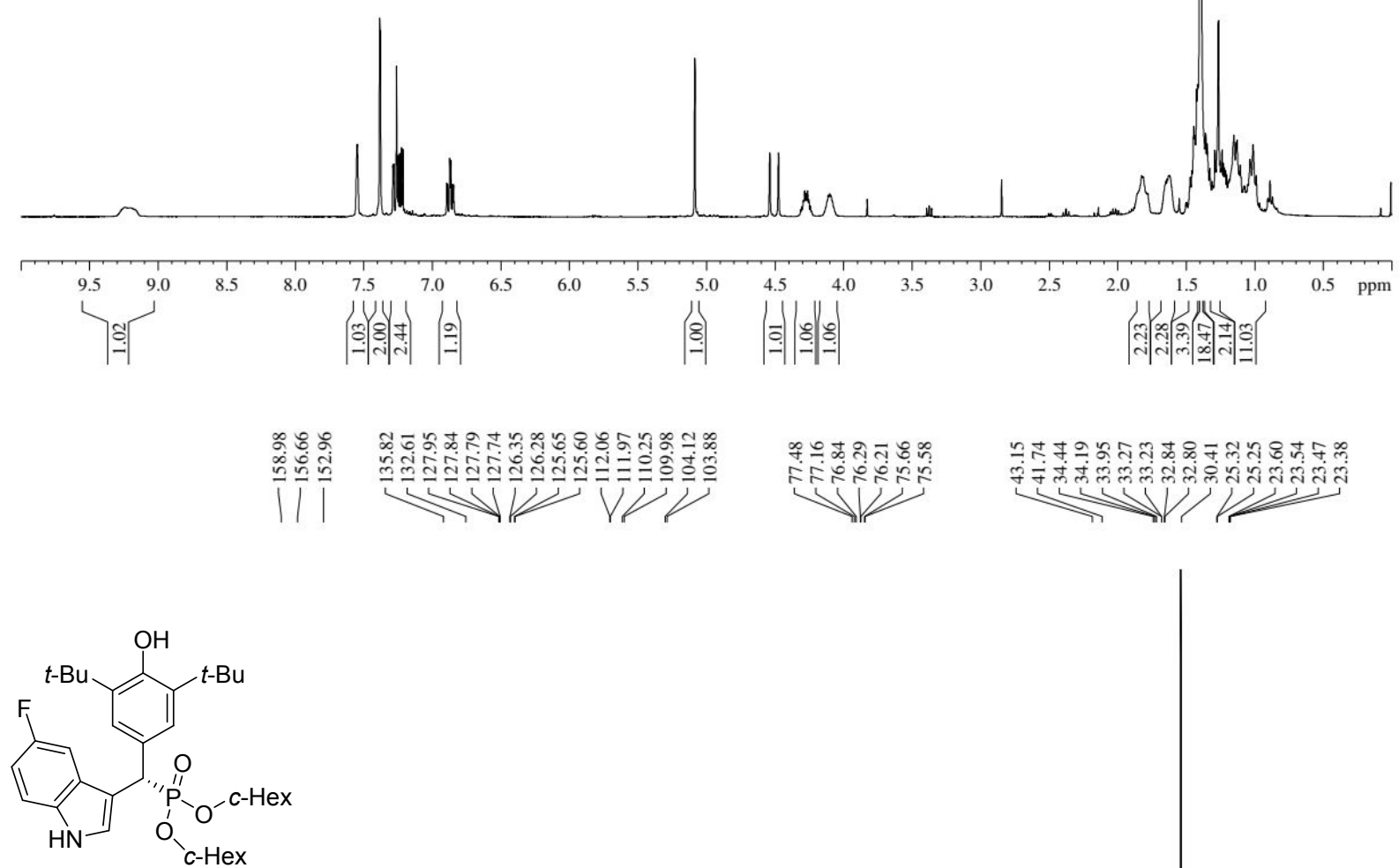

$3 n$

${ }^{13} \mathrm{C}\left\{{ }^{1} \mathrm{H}\right\}-\mathrm{NMR}\left(100 \mathrm{MHz}, \mathrm{CDCl}_{3}\right)$

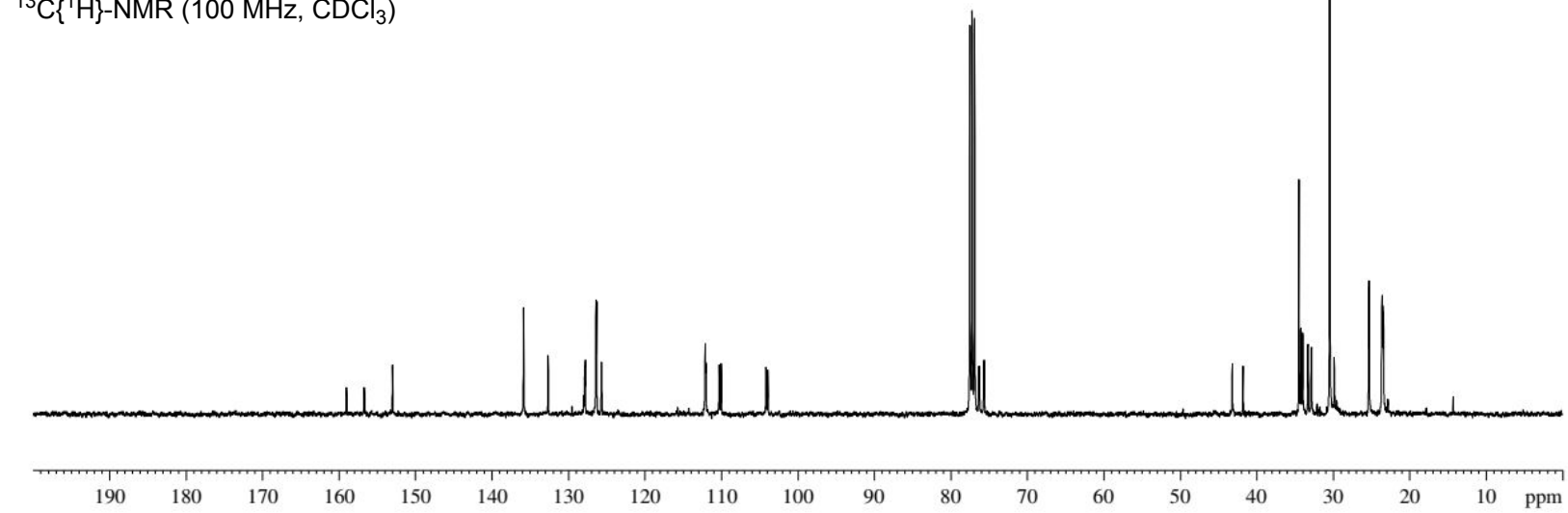




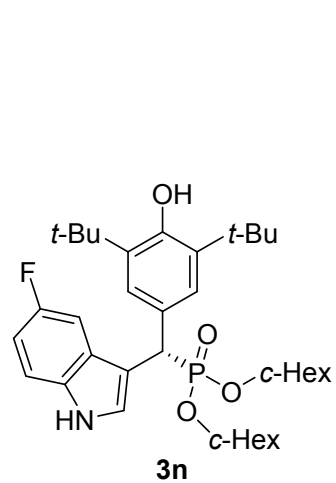

${ }^{19} \mathrm{~F}-\mathrm{NMR}\left(376 \mathrm{MHz}, \mathrm{CDCl}_{3}\right)$
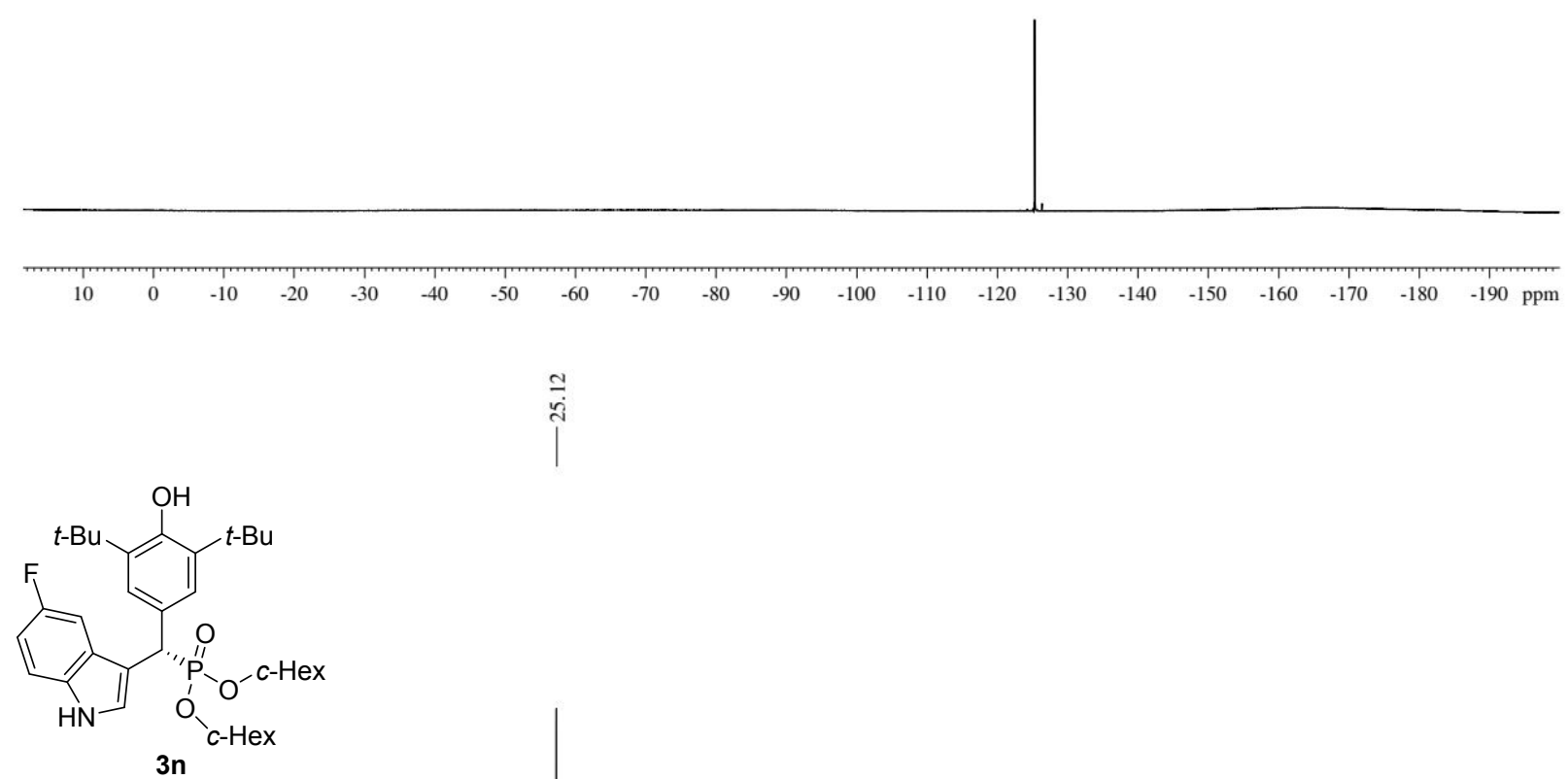

${ }^{31} \mathrm{P}-\mathrm{NMR}\left(162 \mathrm{MHz}, \mathrm{CDCl}_{3}\right)$

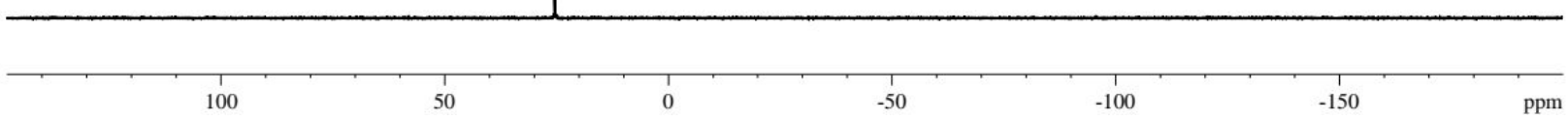


mAU

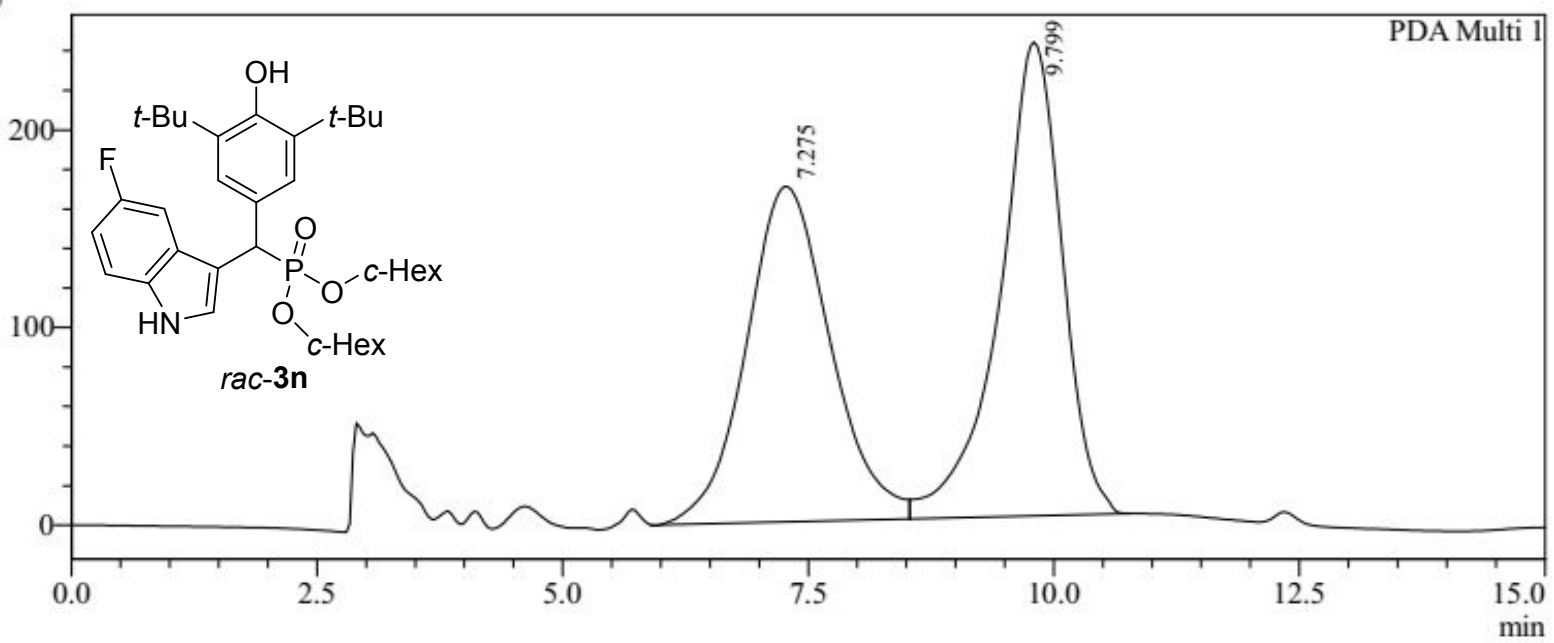

1 PDA Multi $1 / 224 \mathrm{~nm}, 4 \mathrm{~nm}$

\begin{tabular}{|c|c|c|c|c|}
\hline \multicolumn{5}{|c|}{ PDA Chl 224nm } \\
\hline Peak\# & Ret. Time & Area & Height & Area $\%$ \\
\hline 1 & 7.275 & 10103612 & 169661 & 48.745 \\
\hline 2 & 9.799 & 10623790 & 239703 & 51.255 \\
\hline Total & & 20727403 & 409364 & 100.000 \\
\hline
\end{tabular}

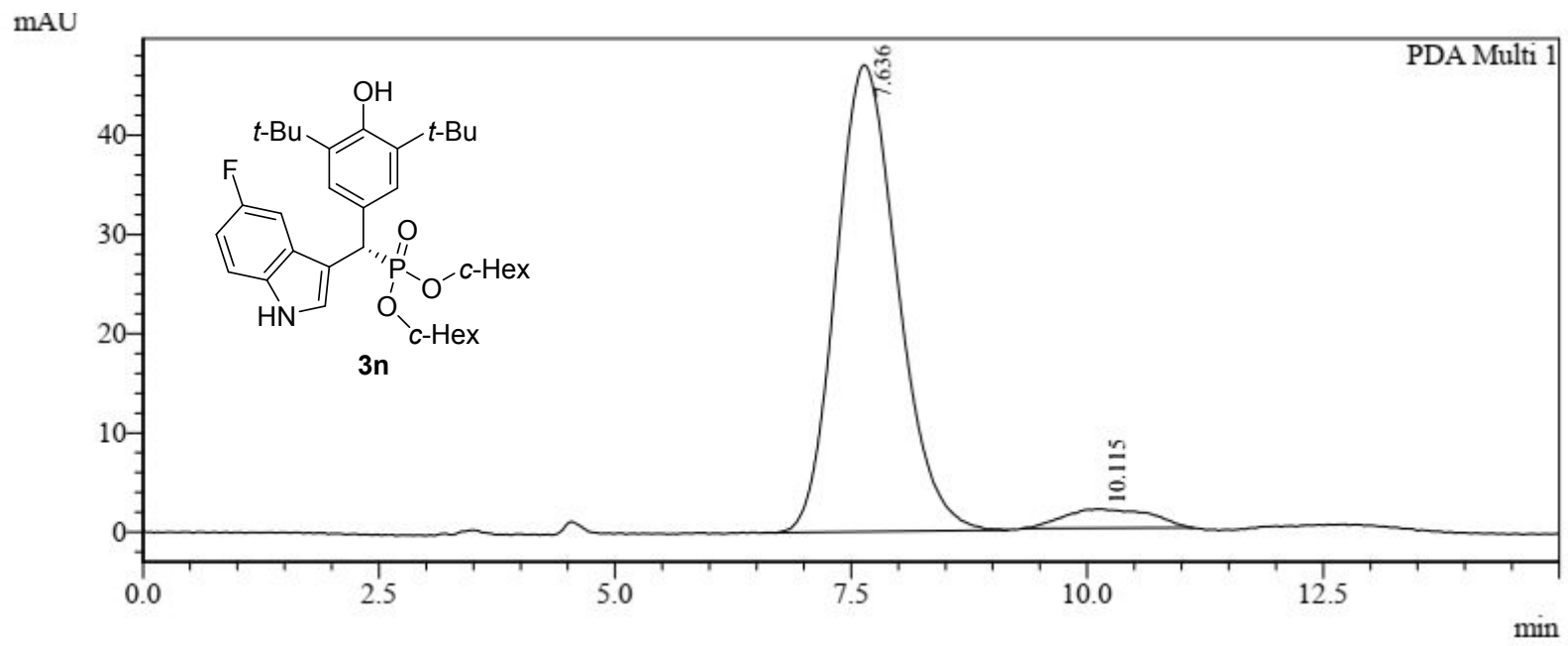

1 PDA Multi $1 / 224 \mathrm{~nm} 4 \mathrm{~nm}$

Ch1 224nm 4nm
\begin{tabular}{|r|r|r|r|r|}
\hline \multicolumn{1}{|c|}{ Peak\# } & Ret. Time & \multicolumn{1}{|c|}{ Area } & \multicolumn{1}{|c|}{ Height } & \multicolumn{1}{c|}{ Area \% } \\
\hline 1 & 7.636 & 2133207 & 47047 & 94.527 \\
\hline 2 & 10.115 & 123514 & 1928 & 5.473 \\
\hline Total & & 2256720 & 48975 & 100.000 \\
\hline
\end{tabular}




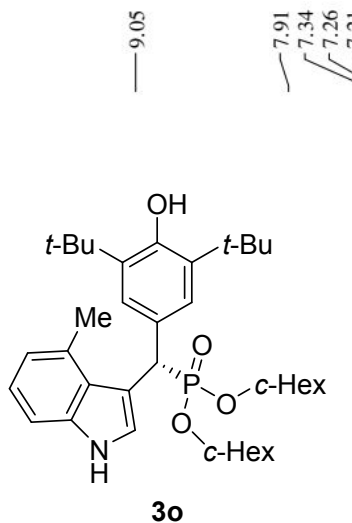

${ }^{1} \mathrm{H}-\mathrm{NMR}\left(400 \mathrm{MHz}, \mathrm{CDCl}_{3}\right)$
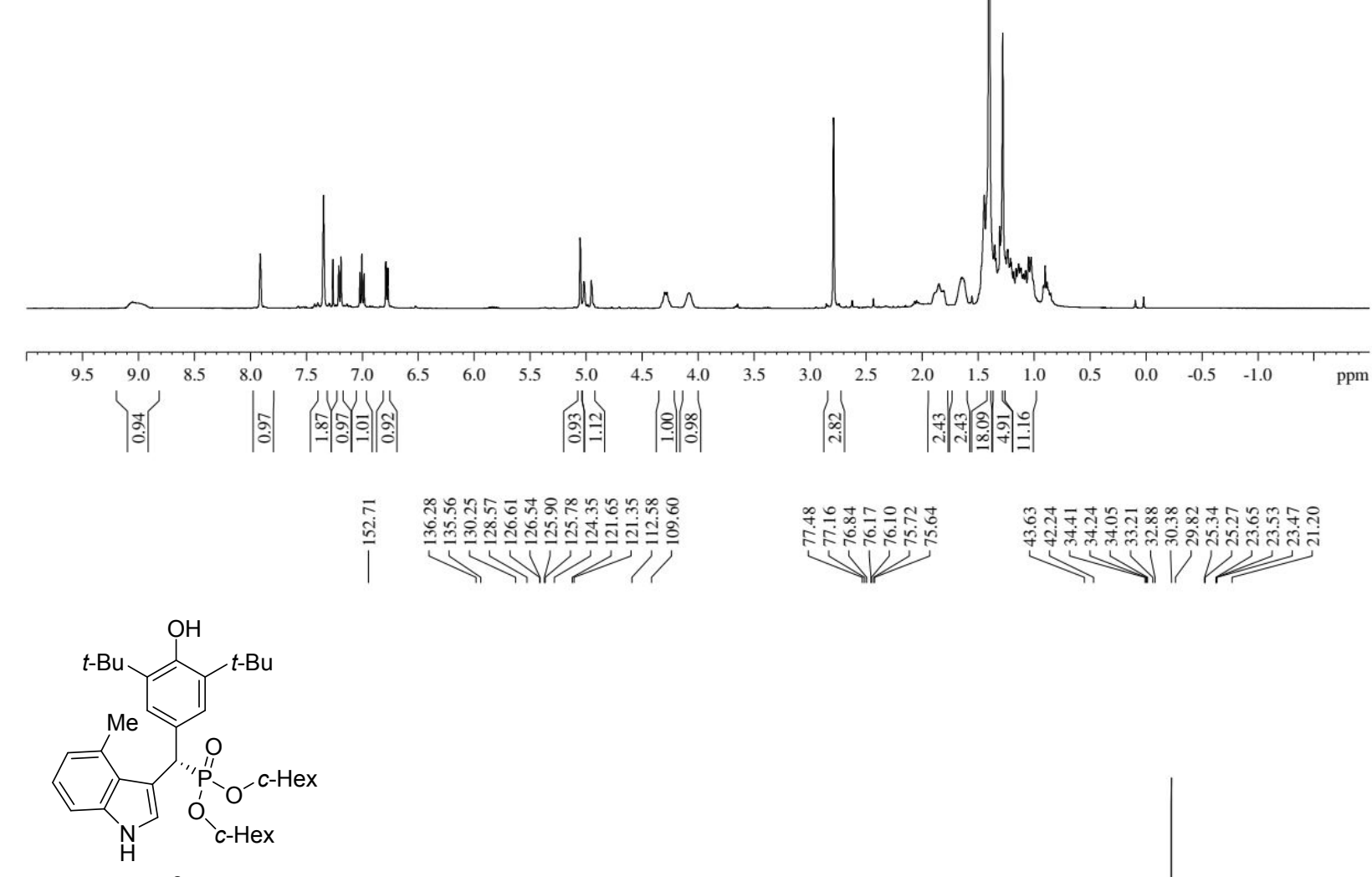

${ }^{13} \mathrm{C}\left\{{ }^{1} \mathrm{H}\right\}-\mathrm{NMR}\left(100 \mathrm{MHz}, \mathrm{CDCl}_{3}\right)$

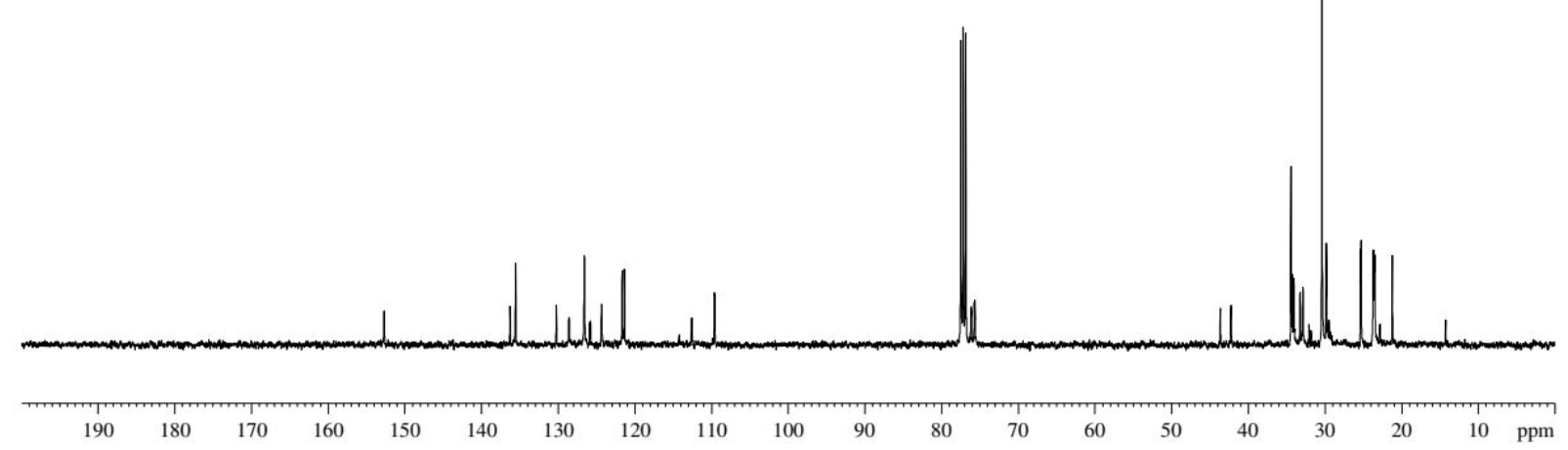




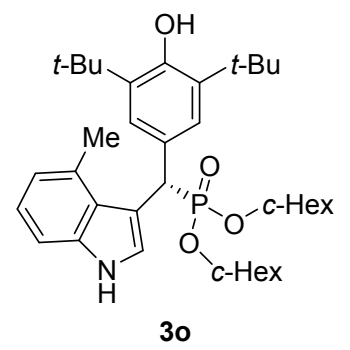

${ }^{31} \mathrm{P}-\mathrm{NMR}\left(162 \mathrm{MHz}, \mathrm{CDCl}_{3}\right.$ )

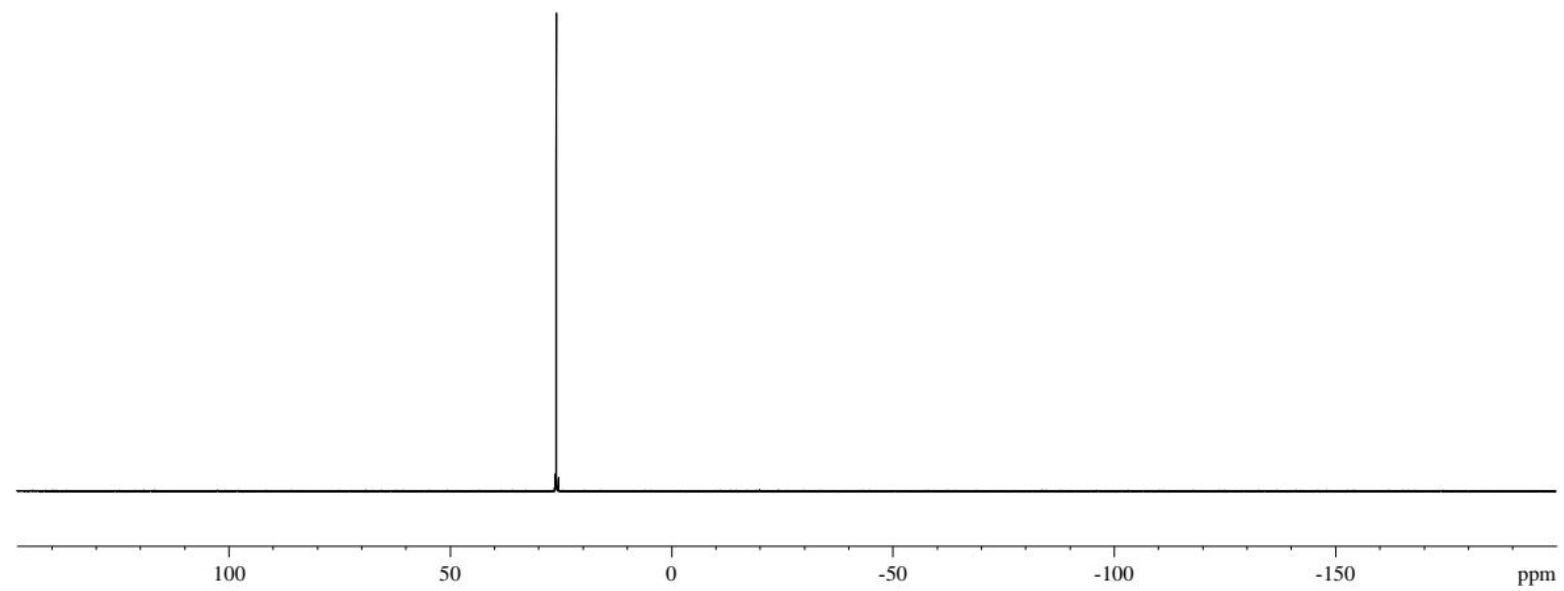

mAU

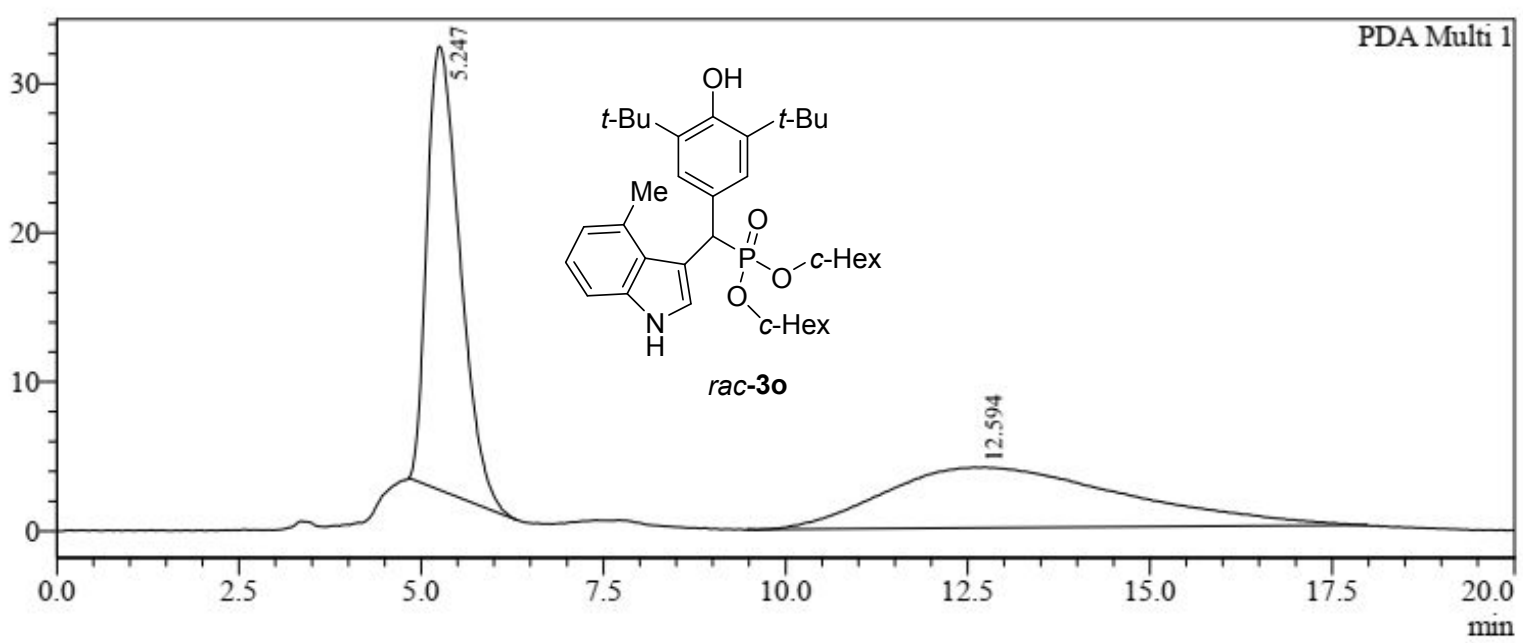

1 PDA Multi $1 / 224 \mathrm{~nm} 4 \mathrm{~nm}$

Ch1 $224 \mathrm{~nm}$ 4nm
\begin{tabular}{|r|r|r|r|r|}
\hline \multicolumn{1}{|c|}{ Peak\# } & Ret. Time & \multicolumn{1}{c|}{ Area } & Height & Area \% \\
\hline 1 & 5.247 & 941716 & 29723 & 50.234 \\
\hline 2 & 12.594 & 932939 & 4067 & 49.766 \\
\hline Total & & 1874655 & 33791 & 100.000
\end{tabular}


mAU

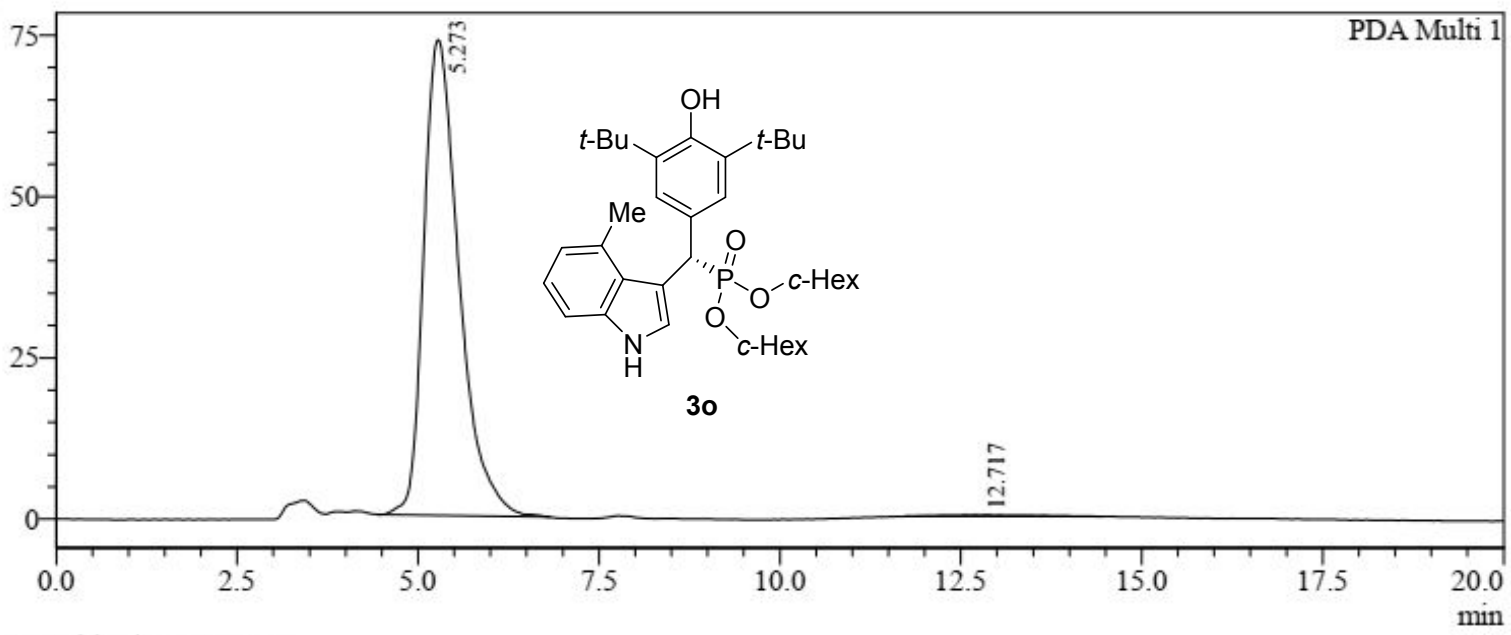

1 PDA Multi $1 / 224 \mathrm{~nm} 4 \mathrm{~nm}$

PeakTable

Ch1 224nm 4nm
\begin{tabular}{|r|r|r|r|r|}
\hline Peak\# & Ret. Time & Area & Height & \multicolumn{1}{|c|}{ Area $\%$} \\
\hline 1 & 5.273 & 2512451 & 73713 & 99.228 \\
\hline 2 & 12.717 & 19557 & 206 & 0.772 \\
\hline Total & & 2532008 & 73919 & 100.000 \\
\hline
\end{tabular}

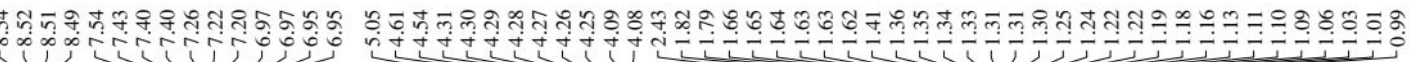<smiles>CCOP(=O)(OCC)C(c1cc(C(C)(C)C)c(O)c(C(C)(C)C)c1)c1c[nH]c2ccc(C)cc12</smiles>

$3 p$

${ }^{1} \mathrm{H}-\mathrm{NMR}\left(400 \mathrm{MHz}, \mathrm{CDCl}_{3}\right)$

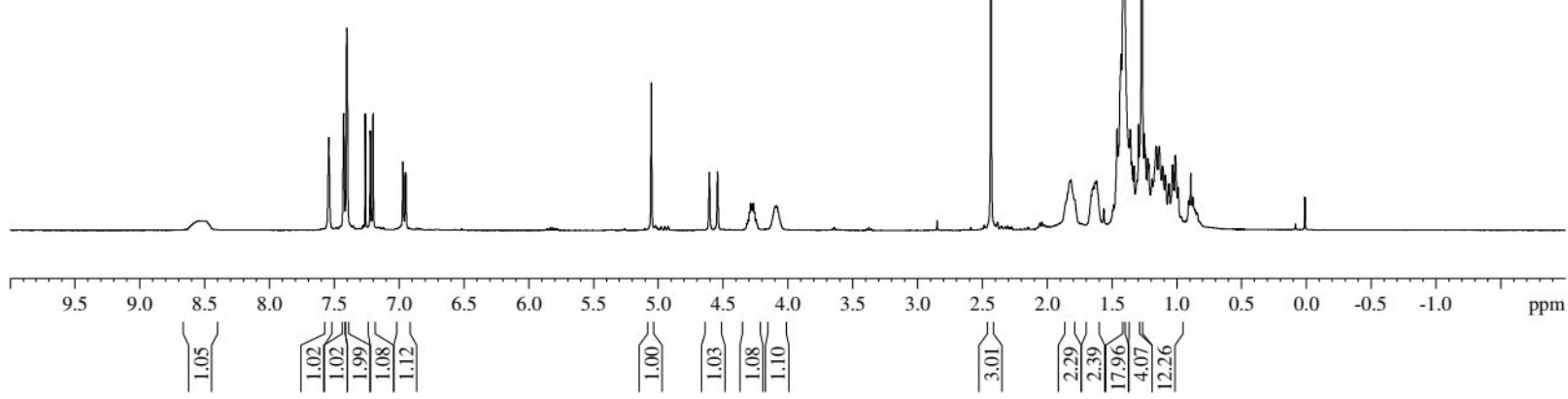



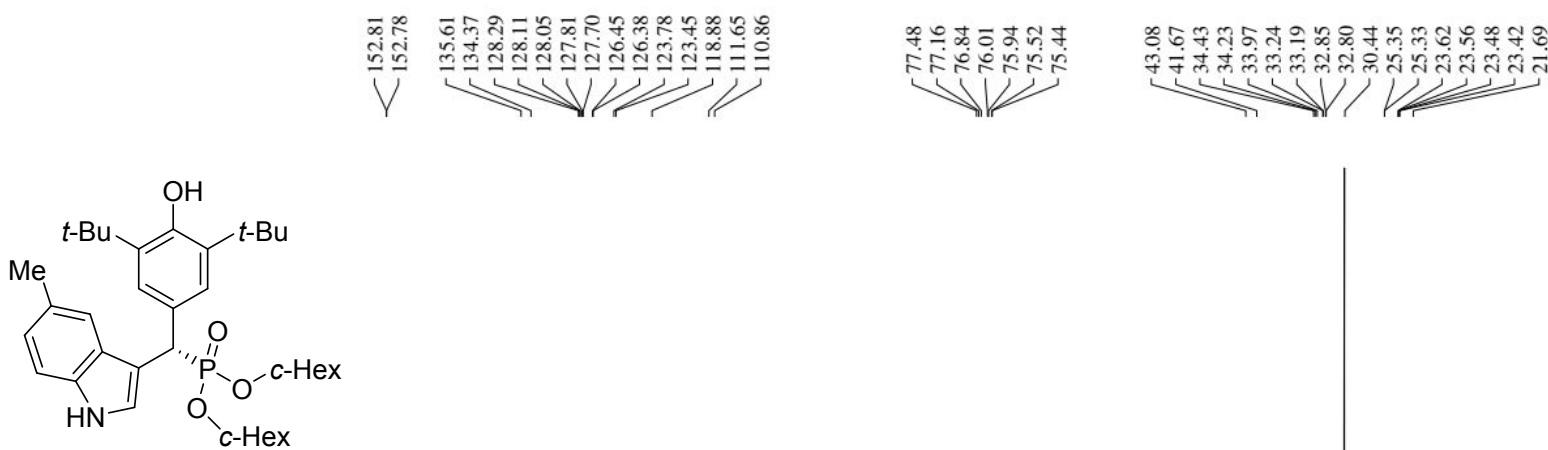

$3 p$

${ }^{13} \mathrm{C}\left\{{ }^{1} \mathrm{H}\right\}-\mathrm{NMR}\left(100 \mathrm{MHz}, \mathrm{CDCl}_{3}\right)$
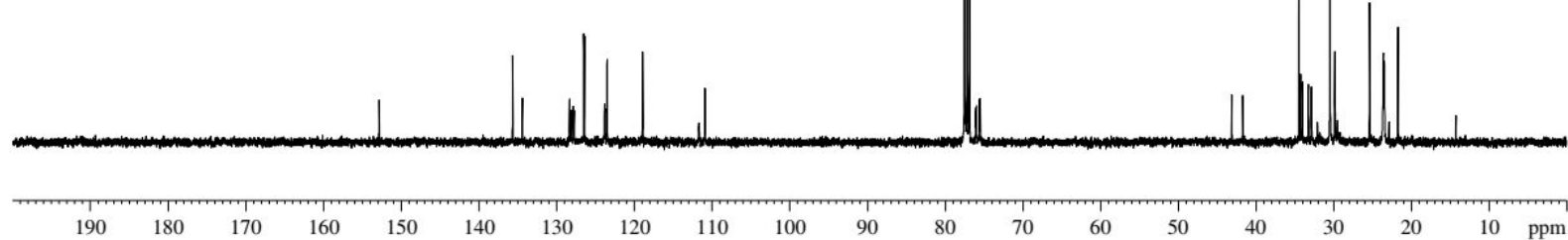

$$
\text { iู่ }
$$<smiles>CCOP(=O)(OC[14CH3])C(c1cc(C(C)(C)C)c(O)c(C(C)(C)C)c1)c1c[nH]c2ccc(C)cc12</smiles>

$3 p$

${ }^{31} \mathrm{P}-\mathrm{NMR}\left(162 \mathrm{MHz}, \mathrm{CDCl}_{3}\right.$ )

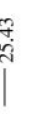


$\mathrm{mAU}$

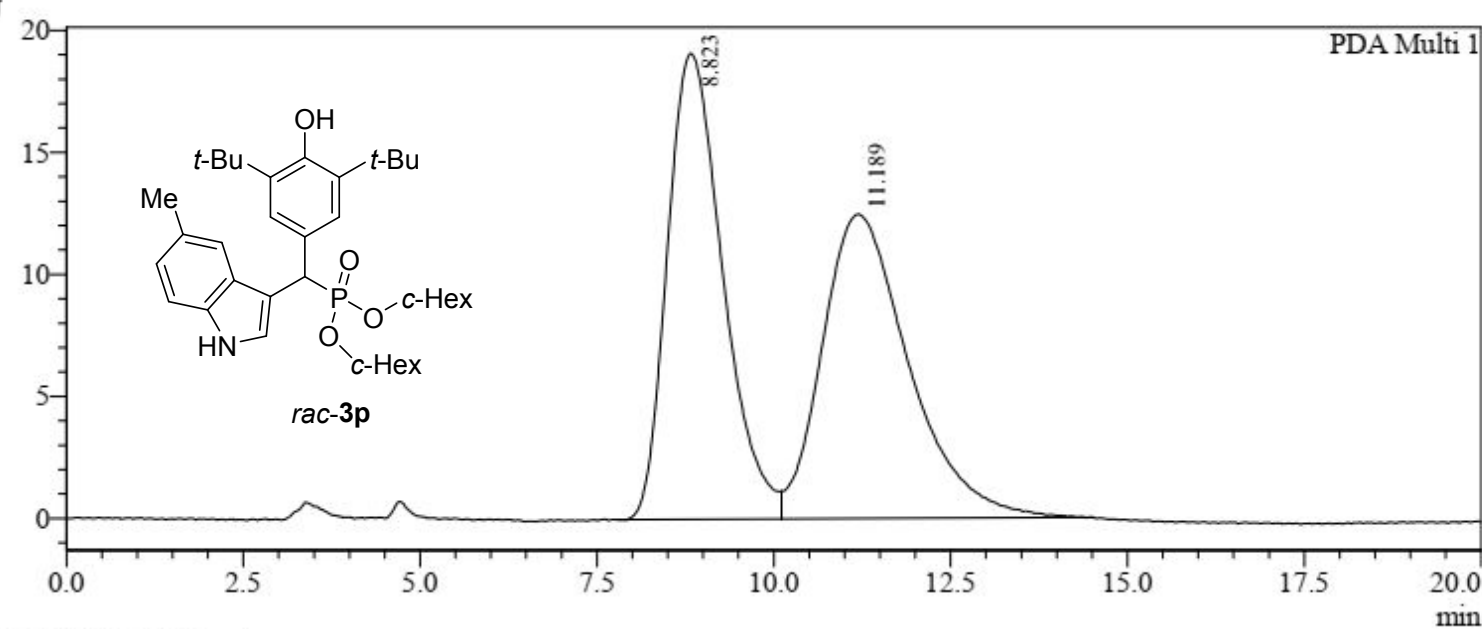

1 PDA Multi $1 / 224 \mathrm{~nm} 4 \mathrm{~nm}$

Ch1 $224 \mathrm{~nm} 4 \mathrm{~nm}$
\begin{tabular}{|r|r|r|r|r|}
\hline \multicolumn{1}{|c|}{ Peak\# } & ReakTable \\
\hline 1 & 8.823 & 1041928 & Height & Area $\%$ \\
\hline 2 & 11.189 & 1061936 & 19081 & 49.524 \\
\hline Total & & 2103865 & 31549 & 50.476 \\
\hline
\end{tabular}

mAU

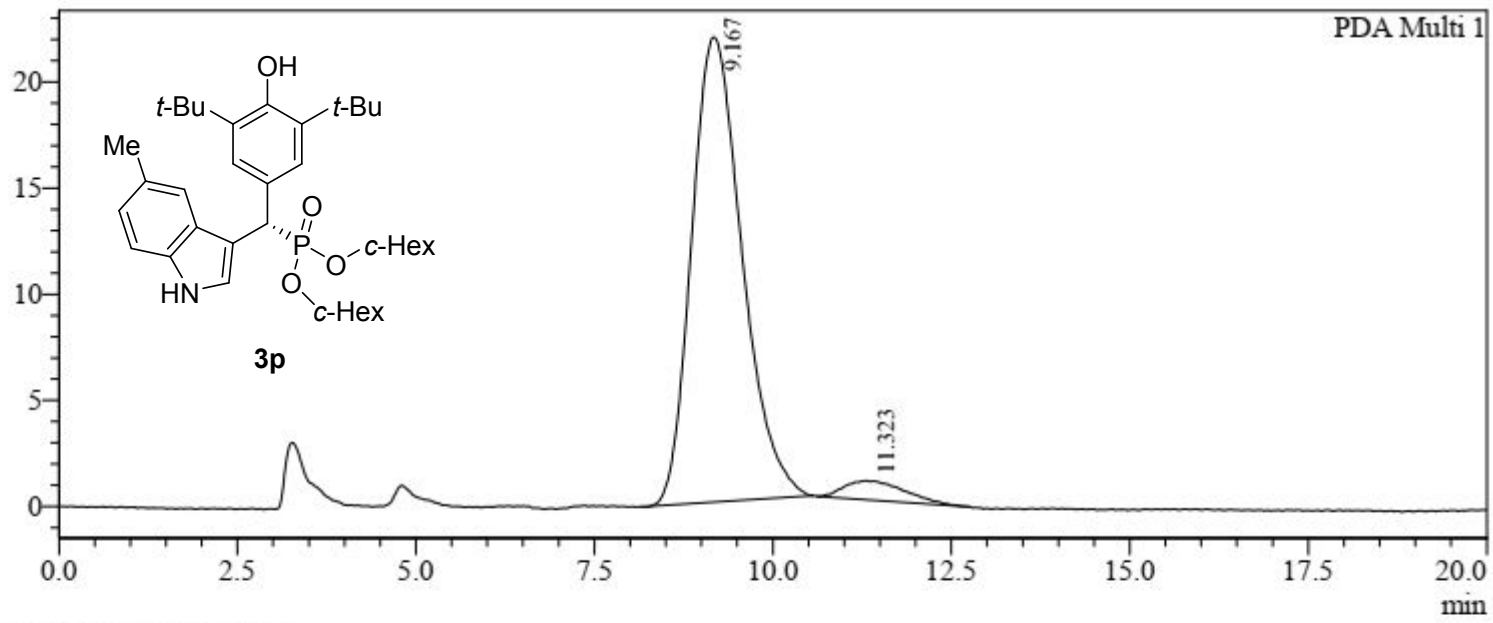

1 PDA Multi $1 / 224 \mathrm{~nm} 4 \mathrm{~nm}$

PeakTable

Ch1 $224 \mathrm{~nm} 4 \mathrm{~nm}$
\begin{tabular}{|r|r|r|r|r|}
\hline \multicolumn{1}{|c|}{ Peak\# } & Ret. Time & \multicolumn{1}{|c|}{ Area } & Height & \multicolumn{1}{c|}{ Area $\%$} \\
\hline 1 & 9.167 & 1078355 & 21907 & 95.208 \\
\hline 2 & 11.323 & 54280 & 899 & 4.792 \\
\hline Total & & 1132635 & 22806 & 100.000 \\
\hline
\end{tabular}


<smiles>CCOP(=O)(OCC)C(c1cc(C(C)(C)C)c(O)c(C(C)(C)C)c1)c1c[nH]c2cc(C)ccc12</smiles>

$3 q$

${ }^{1} \mathrm{H}-\mathrm{NMR}\left(400 \mathrm{MHz}, \mathrm{CDCl}_{3}\right)$
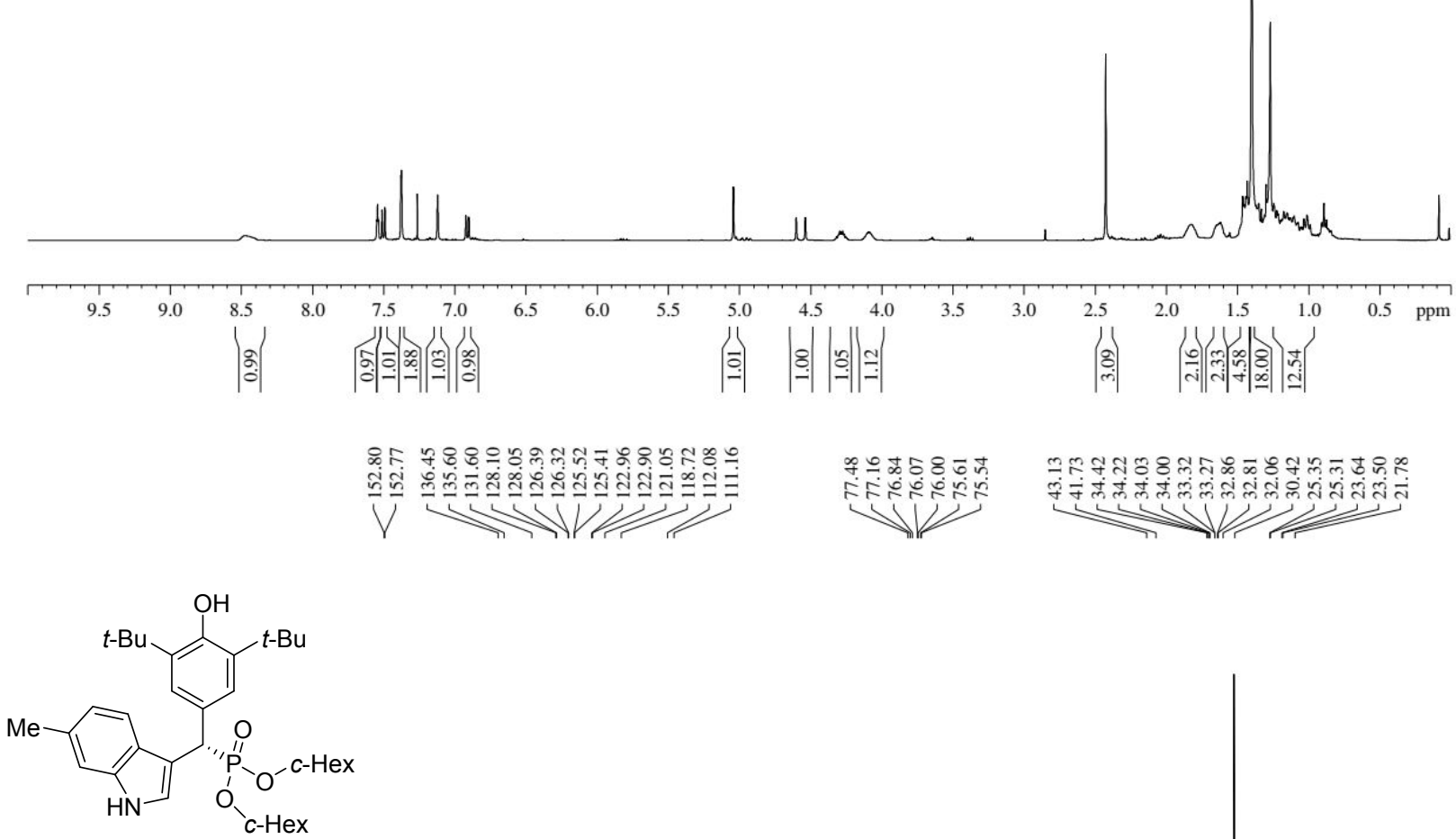

$3 q$

${ }^{13} \mathrm{C}\left\{{ }^{1} \mathrm{H}\right\}-\mathrm{NMR}\left(100 \mathrm{MHz}, \mathrm{CDCl}_{3}\right)$

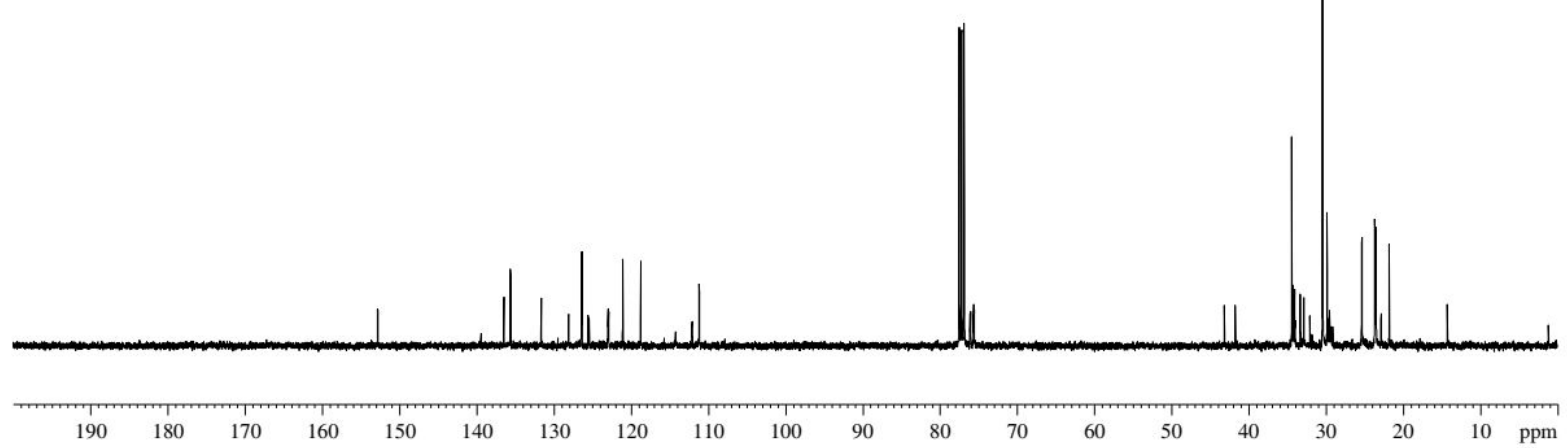




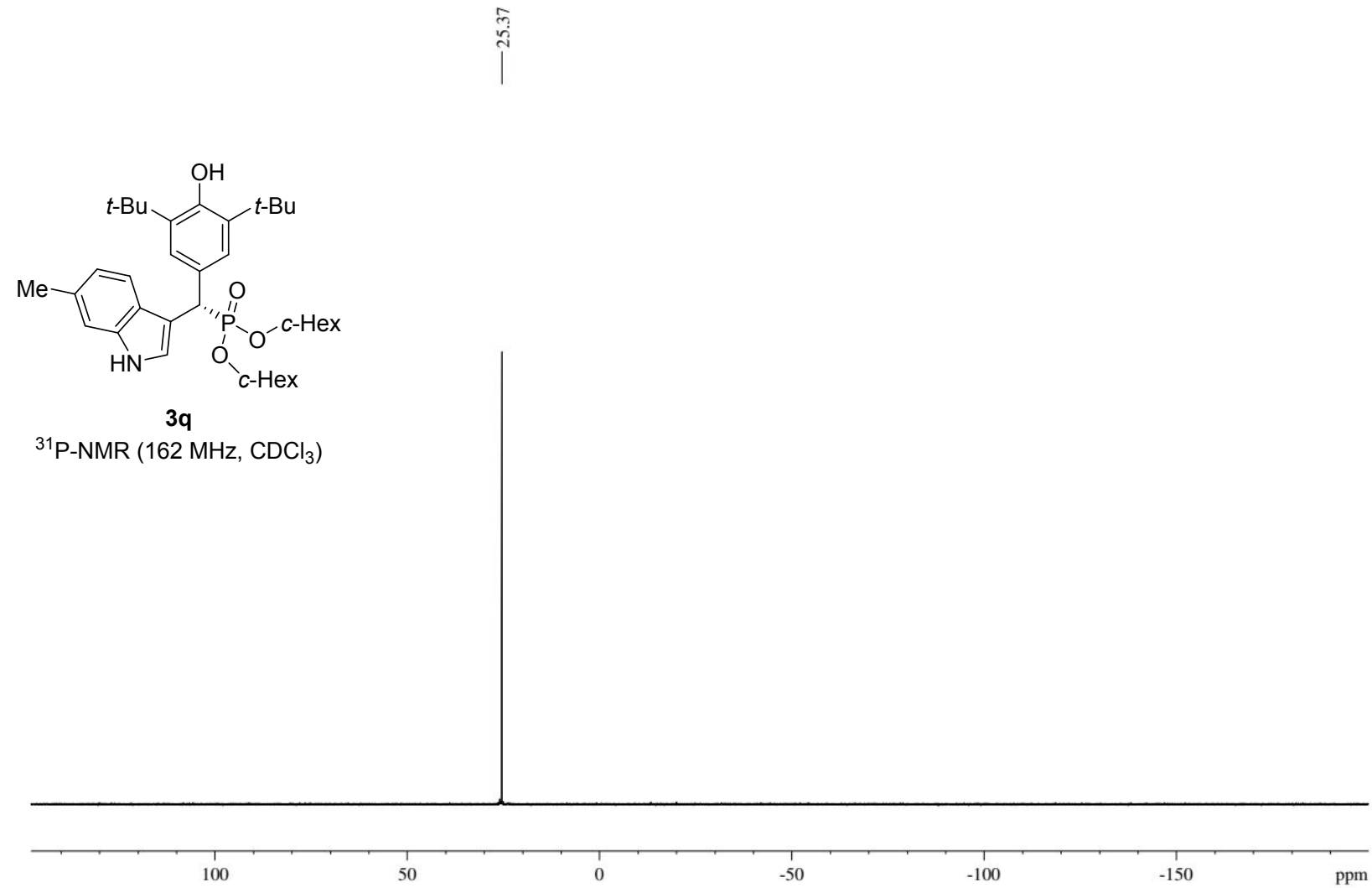

mAU

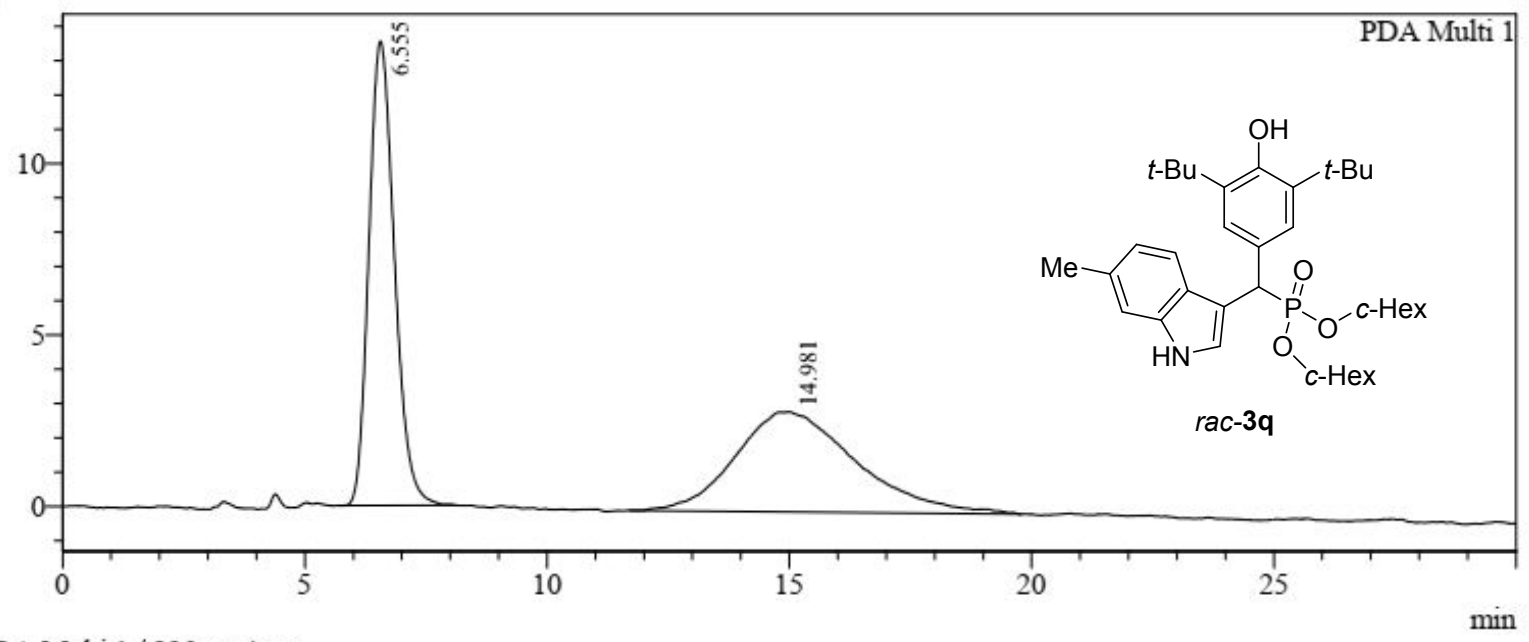

1 PDA Multi $1 / 230 \mathrm{~nm} 4 \mathrm{~nm}$

PeakTable

Ch1 230nm 4nm
\begin{tabular}{|r|r|r|r|r|}
\hline \multicolumn{1}{|c|}{ Peak\# } & Ret. Time & \multicolumn{1}{|c|}{ Area } & Height & \multicolumn{1}{|c|}{ Area $\%$} \\
\hline 1 & 6.555 & 497576 & 13543 & 49.621 \\
\hline 2 & 14.981 & 505180 & 2919 & 50.379 \\
\hline Total & & 1002756 & 16462 & 100.000 \\
\hline
\end{tabular}


mAU

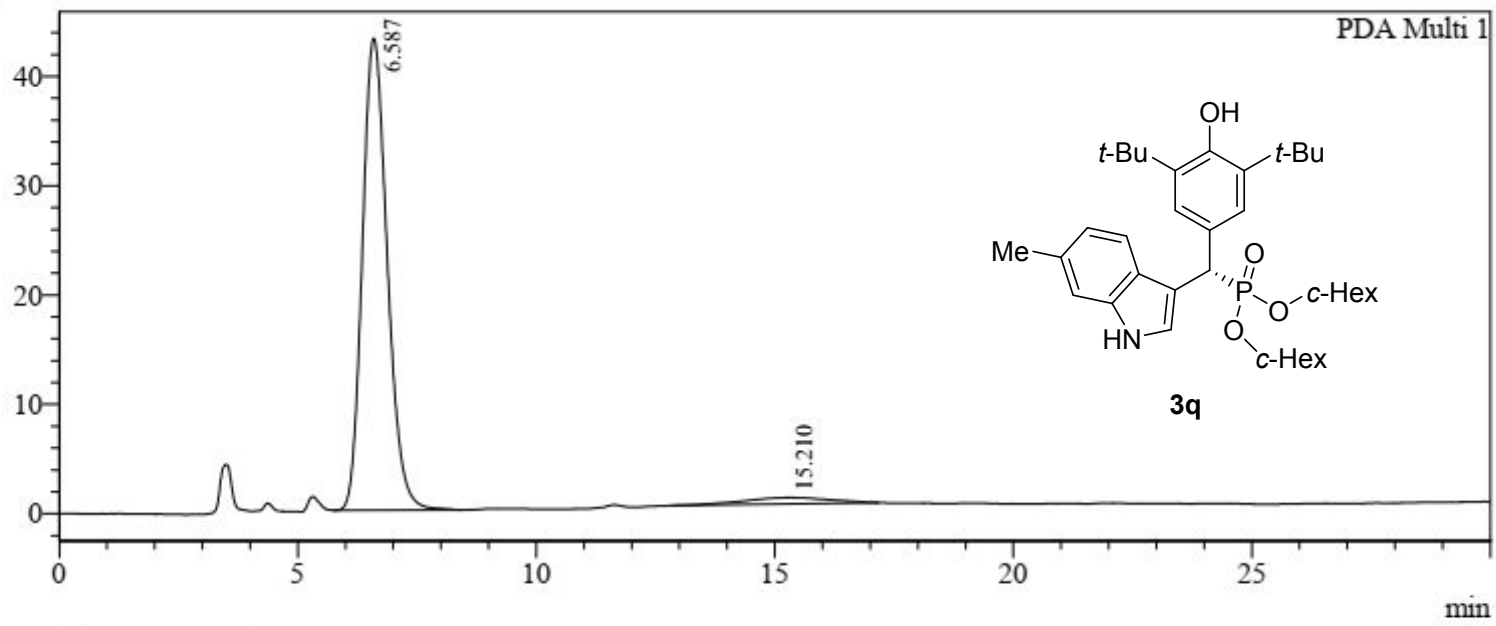

1 PDA Multi $1 / 230 \mathrm{~nm} 4 \mathrm{~nm}$

PeakTable

Ch1 230nm 4nm
\begin{tabular}{|r|r|r|r|r|}
\hline \multicolumn{1}{|c|}{ Peak\# } & Ret. Time & \multicolumn{1}{|c|}{ Area } & \multicolumn{1}{c|}{ Height } & Area \% \\
\hline 1 & 6.587 & 1568415 & 43165 & 95.631 \\
\hline 2 & 15.210 & 71653 & 578 & 4.369 \\
\hline Total & & 1640068 & 43744 & 100.000 \\
\hline
\end{tabular}<smiles>[R]COP(=O)(OC[R])[C@H](c1cc(C(C)(C)C)c(O)c(C(C)(C)C)c1)c1c[nH]c2ccc(OC)cc12</smiles>

${ }^{1} \mathrm{H}-\mathrm{NMR}\left(400 \mathrm{MHz}, \mathrm{CDCl}_{3}\right)$

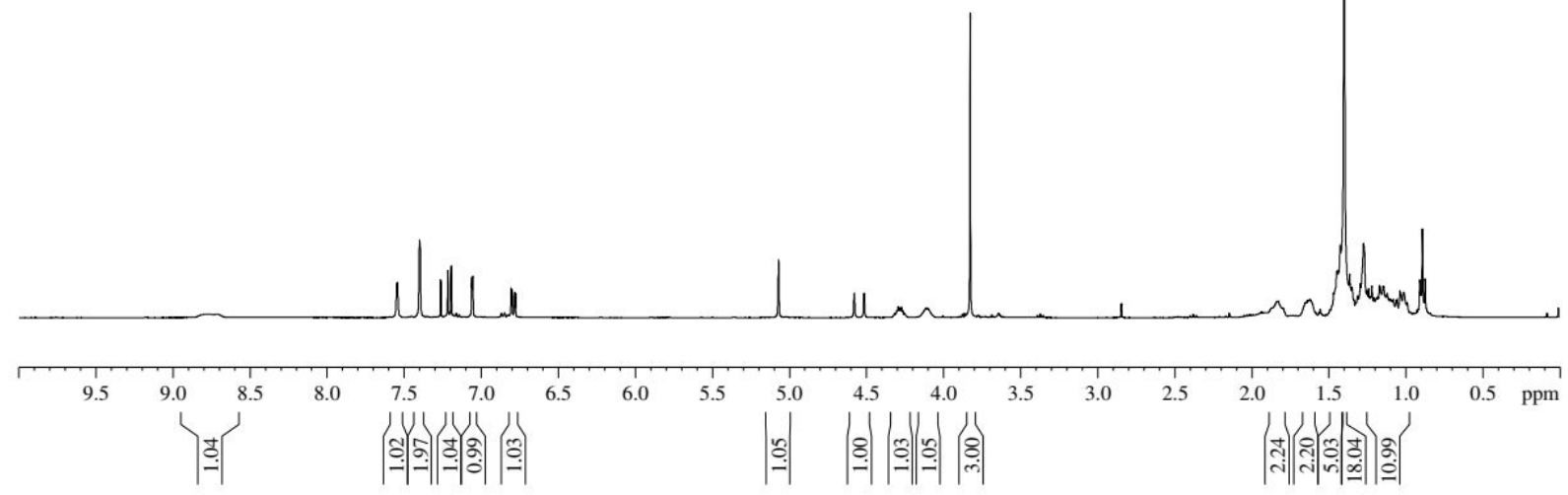



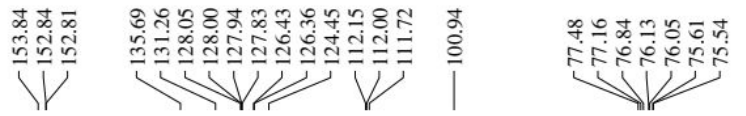

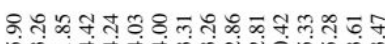

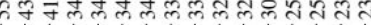

$1+$

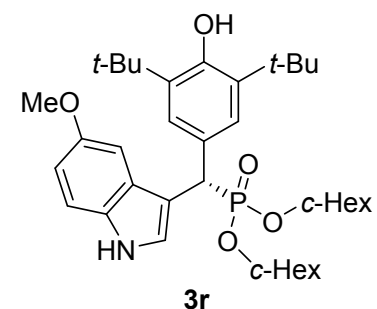

${ }^{13} \mathrm{C}\left\{{ }^{1} \mathrm{H}\right\}-\mathrm{NMR}\left(100 \mathrm{MHz}, \mathrm{CDCl}_{3}\right)$
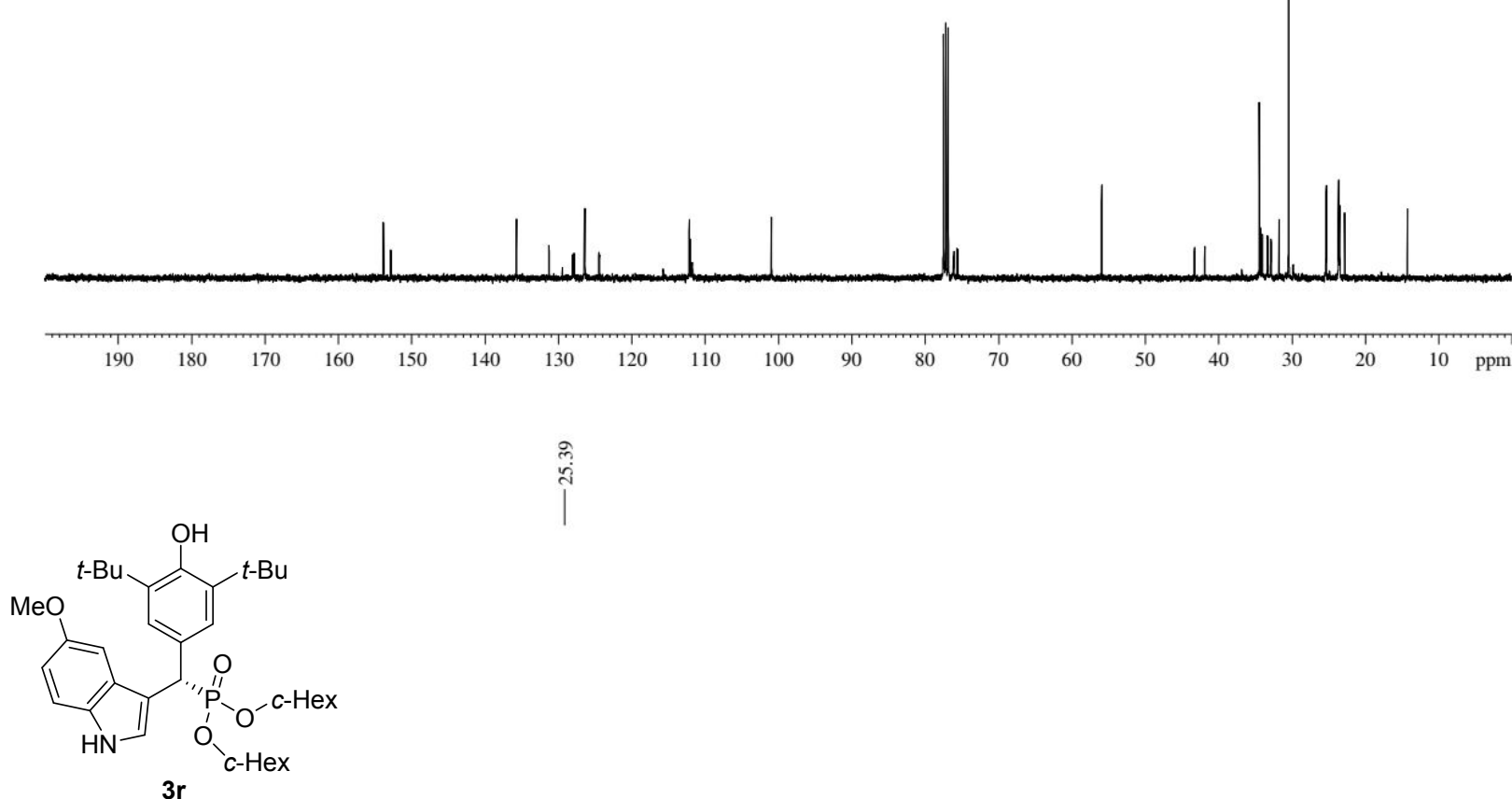

กั่

${ }^{31} \mathrm{P}-\mathrm{NMR}\left(162 \mathrm{MHz}, \mathrm{CDCl}_{3}\right.$ )

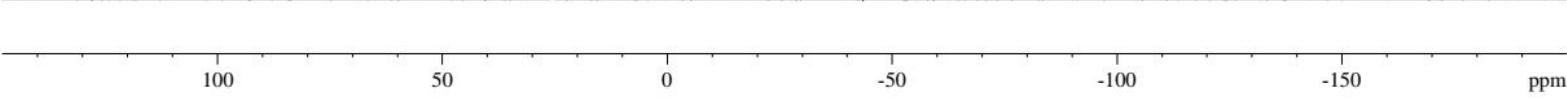


mAU

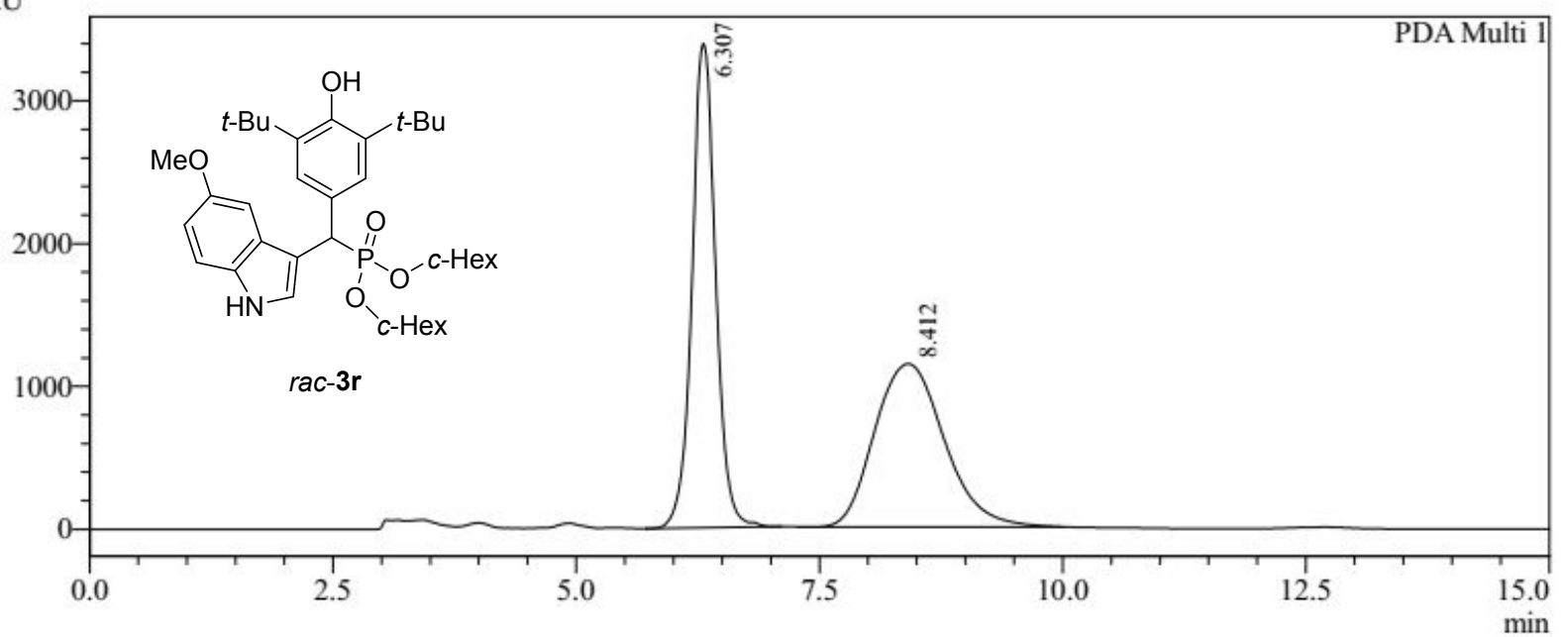

1 PDA Multi $1 / 230 \mathrm{~nm}, 4 \mathrm{~nm}$

\begin{tabular}{|c|c|c|c|c|}
\hline \multicolumn{5}{|c|}{ PDA Chl $230 \mathrm{~nm}$} \\
\hline Peak\# & Ret. Time & Area & Height & Area $\%$ \\
\hline 1 & 6.307 & 57269104 & 3387872 & 50.183 \\
\hline 2 & 8.412 & 56851501 & 1141245 & 49.817 \\
\hline Total & & 114120605 & 4529117 & 100.000 \\
\hline
\end{tabular}

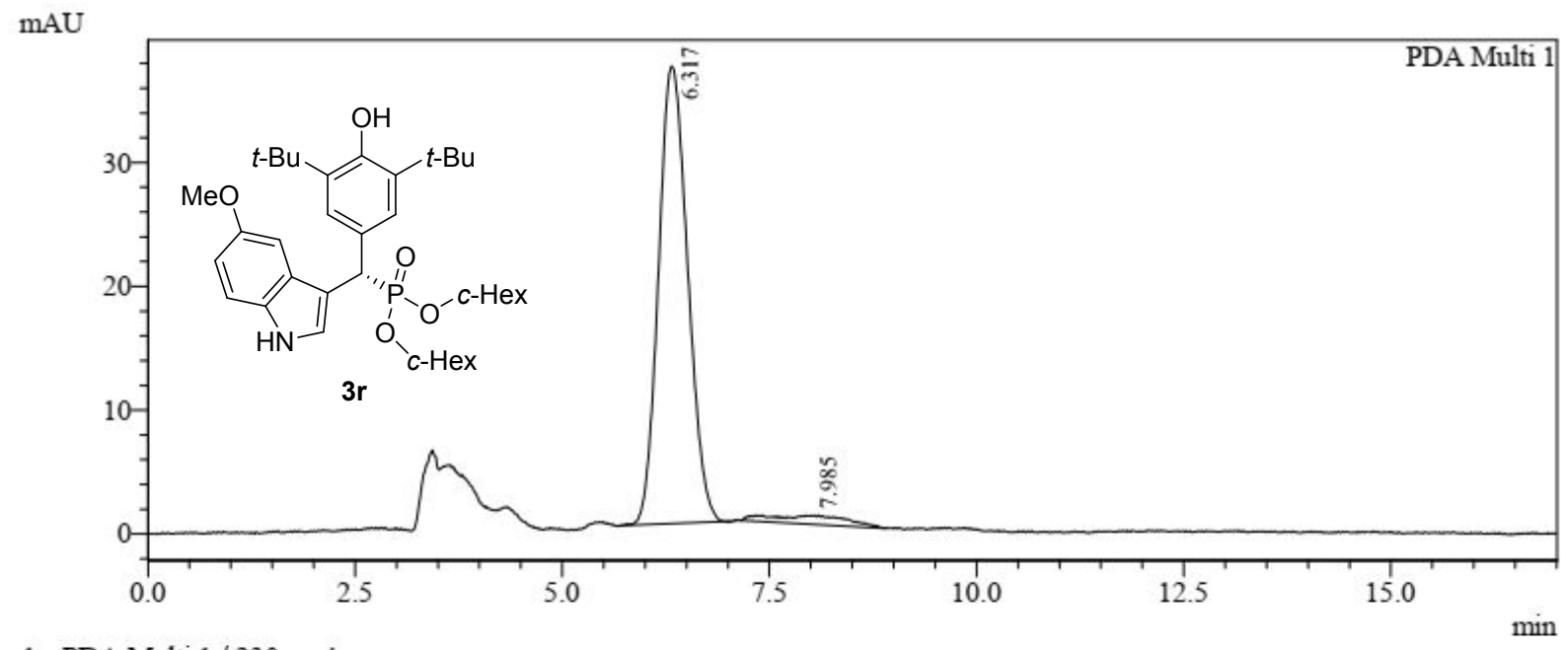

1 PDA Multi $1 / 230 \mathrm{~nm} 4 \mathrm{~nm}$

PeakTable

Ch1 $230 \mathrm{~nm} 4 \mathrm{~nm}$
\begin{tabular}{|r|r|r|r|r|}
\hline \multicolumn{1}{|c|}{ Peak\# } & Ret. Time & \multicolumn{1}{|c|}{ Area } & Height & Area $\%$ \\
\hline 1 & 6.317 & 904895 & 36939 & 95.147 \\
\hline 2 & 7.985 & 46151 & 728 & 4.853 \\
\hline Total & & 951046 & 37667 & 100.000 \\
\hline
\end{tabular}




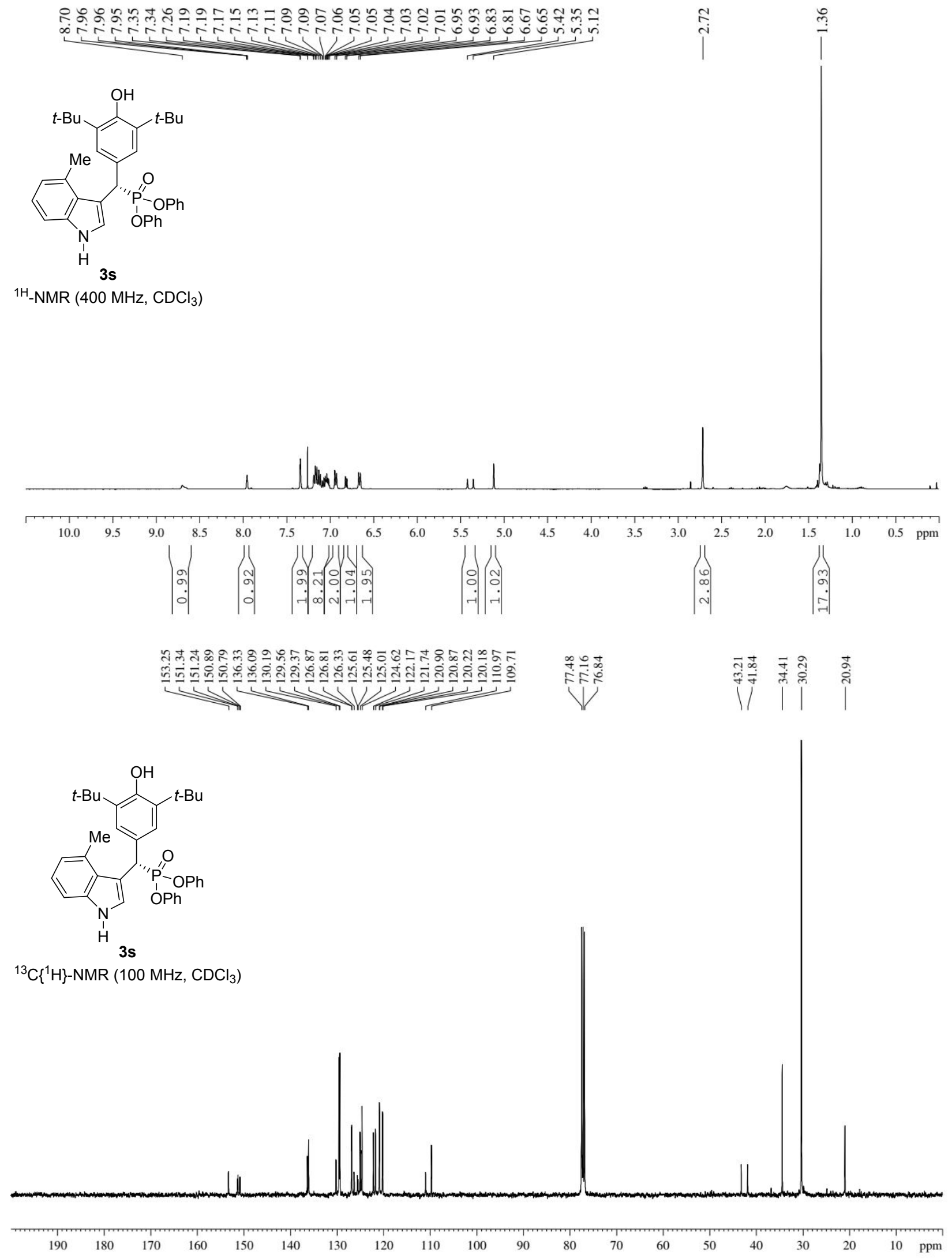




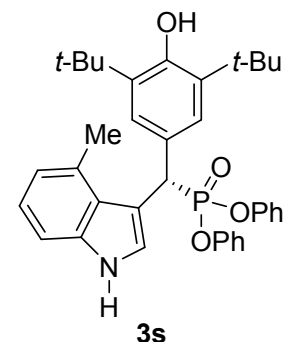

${ }^{31} \mathrm{P}-\mathrm{NMR}\left(162 \mathrm{MHz}, \mathrm{CDCl}_{3}\right)$

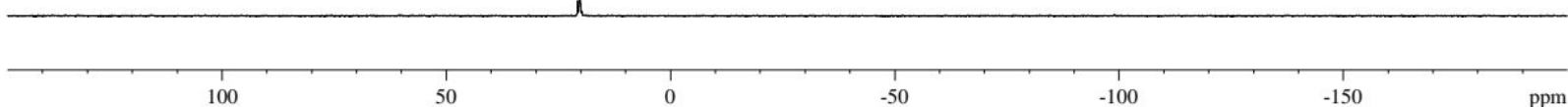

mAU

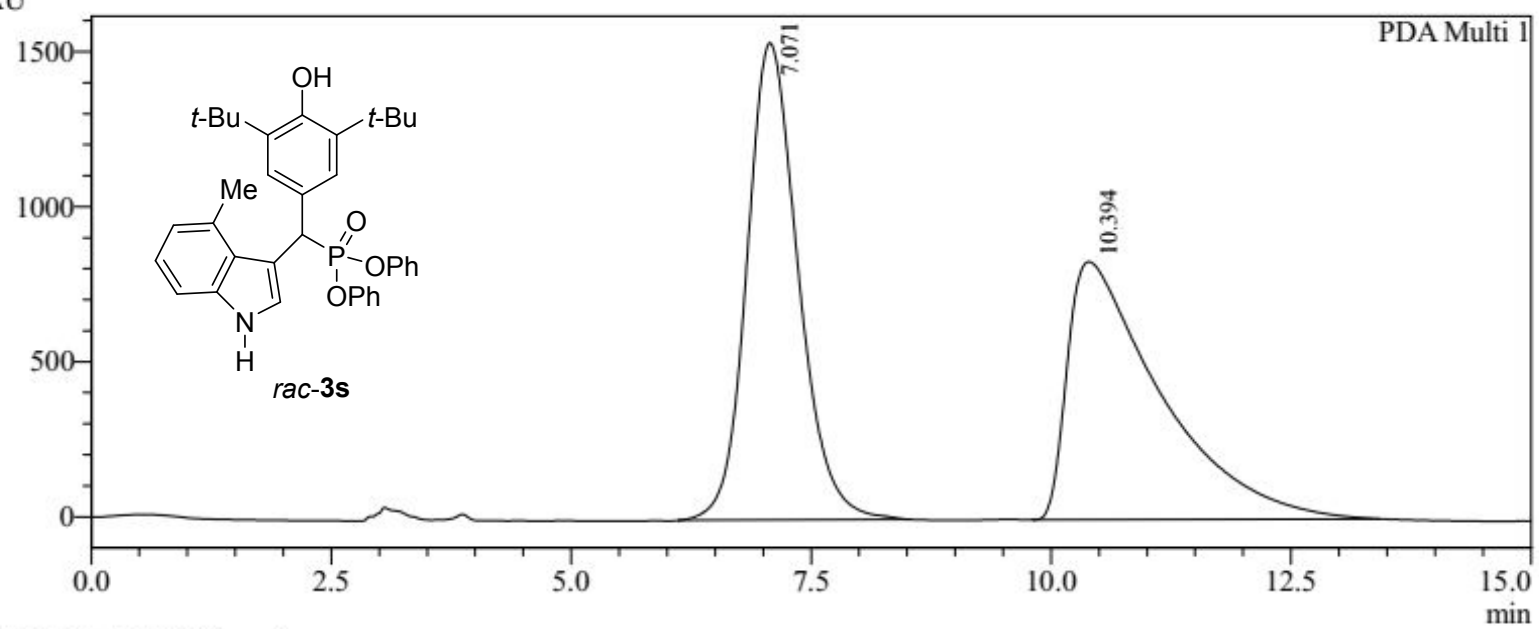

1 PDA Multi $1 / 224 \mathrm{~nm}, 4 \mathrm{~nm}$

\begin{tabular}{|c|c|c|c|c|}
\hline \multicolumn{5}{|c|}{ PDA Chl 224nm } \\
\hline Peak\# & Ret. Time & Area & Height & Area \% \\
\hline 1 & 7.071 & 57363907 & 1538676 & 50.520 \\
\hline 2 & 10.394 & 56182706 & 831664 & 49.480 \\
\hline Total & & 113546613 & 2370340 & 100.000 \\
\hline
\end{tabular}




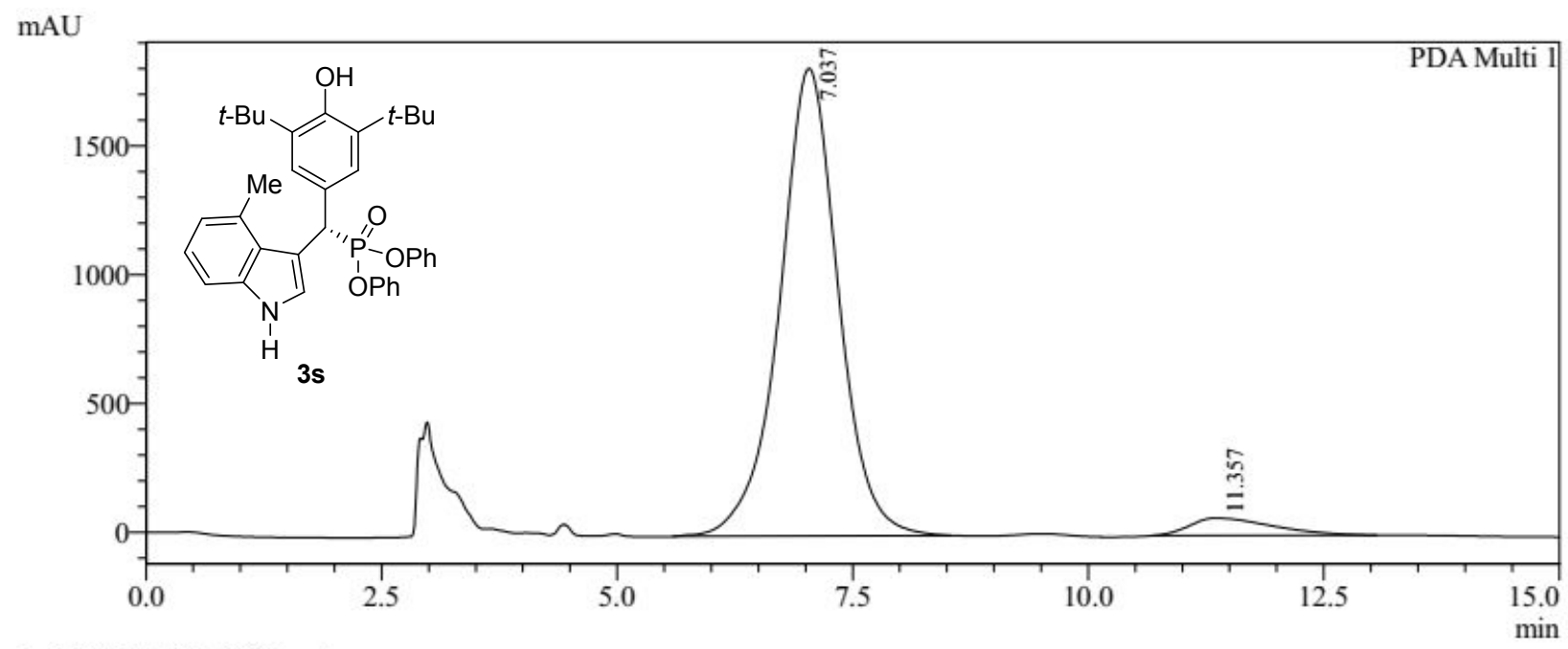

1 PDA Multi $1 / 224 \mathrm{~nm}, 4 \mathrm{~nm}$

\begin{tabular}{|c|c|c|c|c|}
\hline \multicolumn{5}{|c|}{ PDA Chl 224nm } \\
\hline Peak\# & Ret. Time & Area & Height & Area $\%$ \\
\hline 1 & 7.037 & 78730151 & 1815003 & 95.012 \\
\hline 2 & 11.357 & 4133072 & 67693 & 4.988 \\
\hline Total & & 82863223 & 1882696 & 100.000 \\
\hline
\end{tabular}

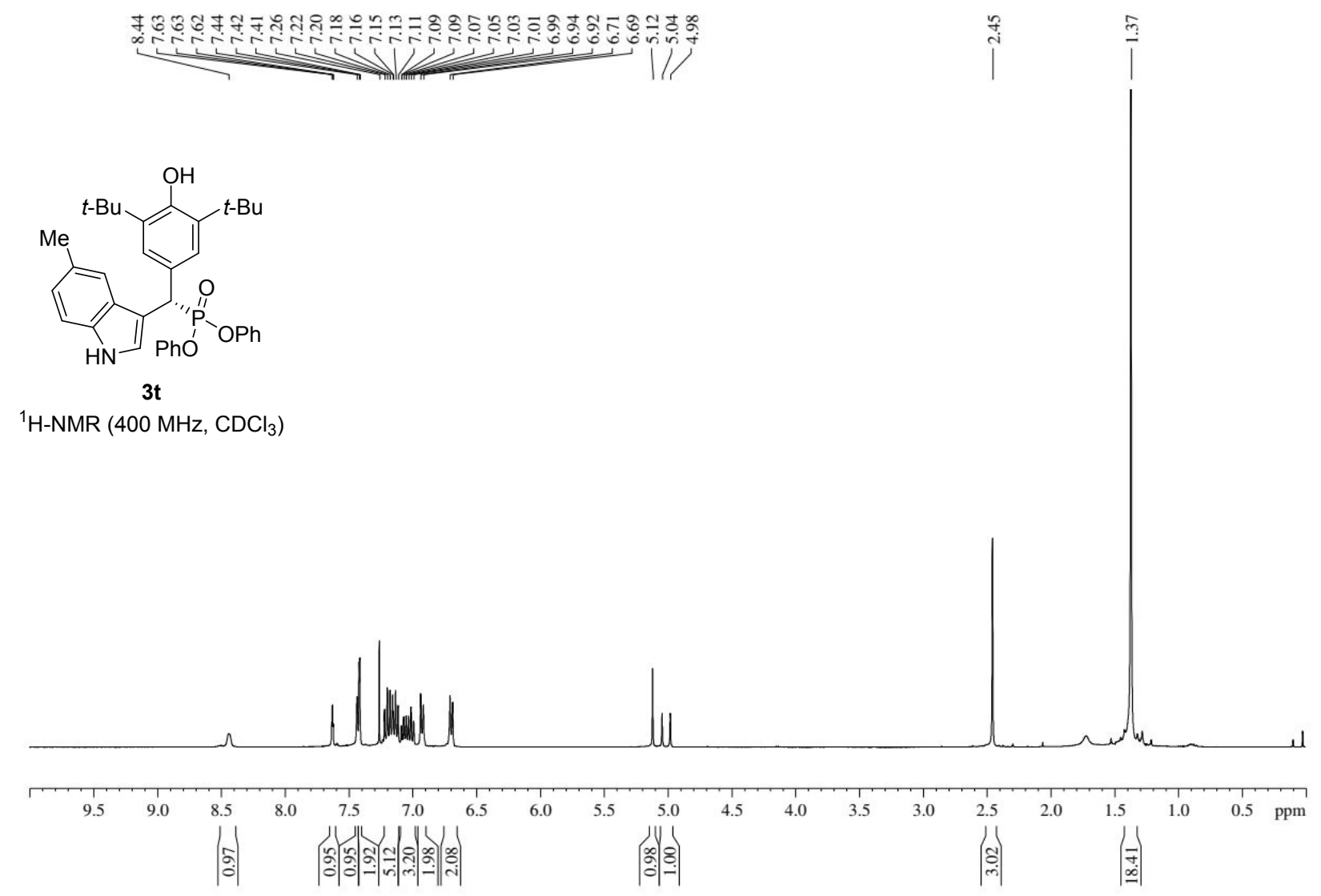



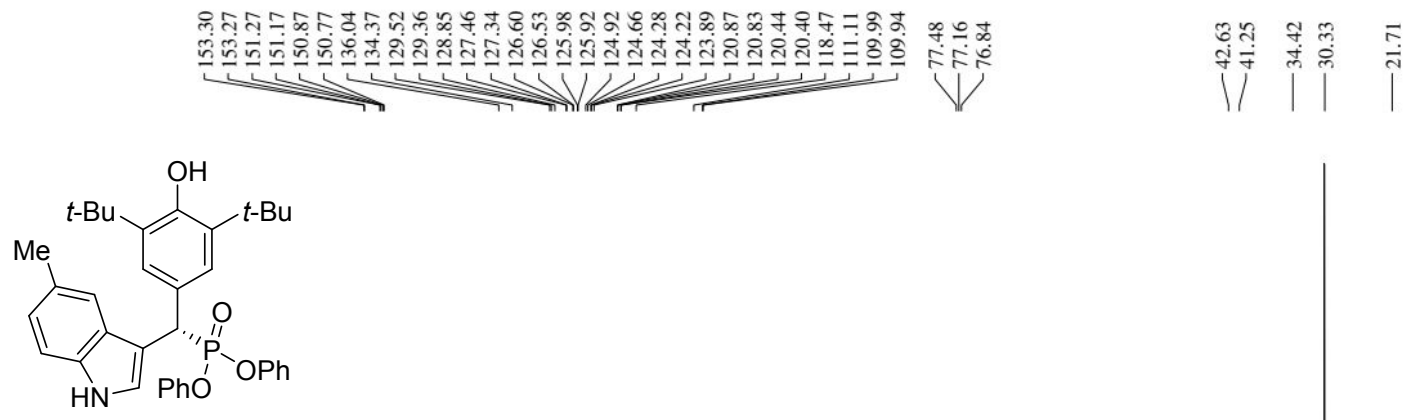

$3 \mathbf{t}$

${ }^{13} \mathrm{C}\left\{{ }^{1} \mathrm{H}\right\}-\mathrm{NMR}\left(100 \mathrm{MHz}, \mathrm{CDCl}_{3}\right)$
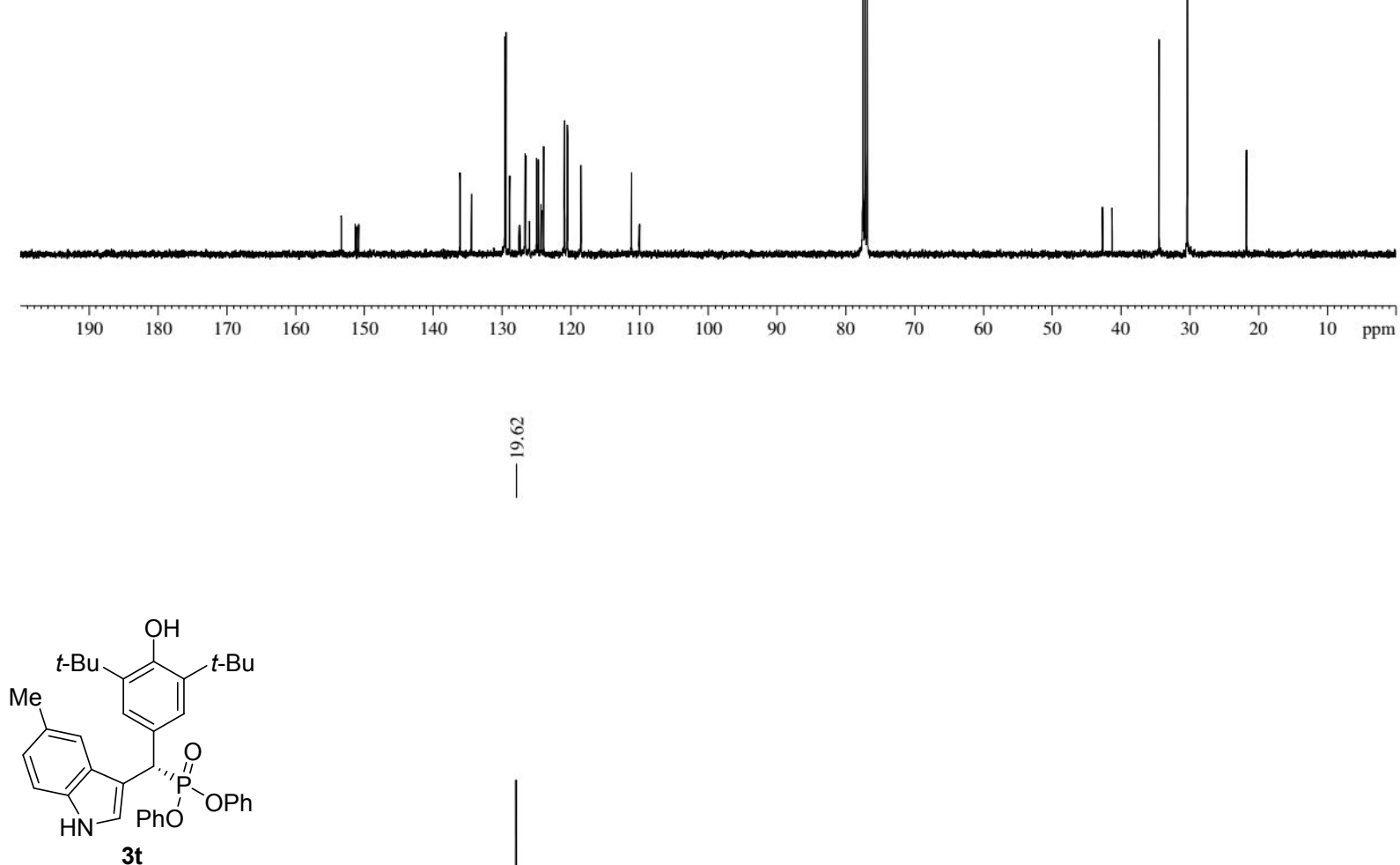

${ }^{31} \mathrm{P}-\mathrm{NMR}\left(162 \mathrm{MHz}, \mathrm{CDCl}_{3}\right.$ ) 


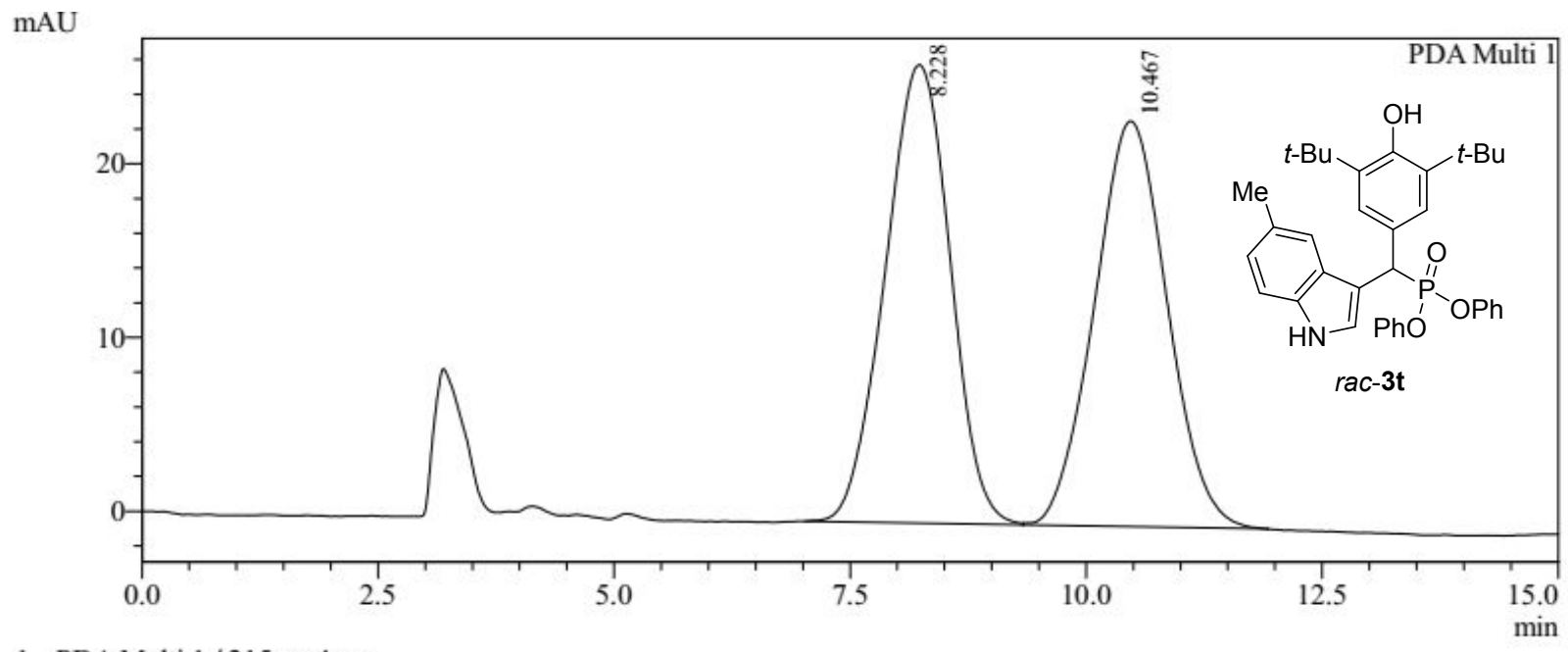

1 PDA Multi $1 / 215 \mathrm{~nm}, 4 \mathrm{~nm}$

PDA Ch1 $215 \mathrm{~nm}$
\begin{tabular}{|r|r|r|r|r|}
\hline \multicolumn{1}{|c|}{ Peak\# } & Ret. Time & Area & Height & Area $\%$ \\
\hline 1 & 8.228 & 1271436 & 26485 & 50.258 \\
\hline 2 & 10.467 & 1258388 & 23434 & 49.742 \\
\hline Total & & 2529824 & 49918 & 100.000 \\
\hline
\end{tabular}

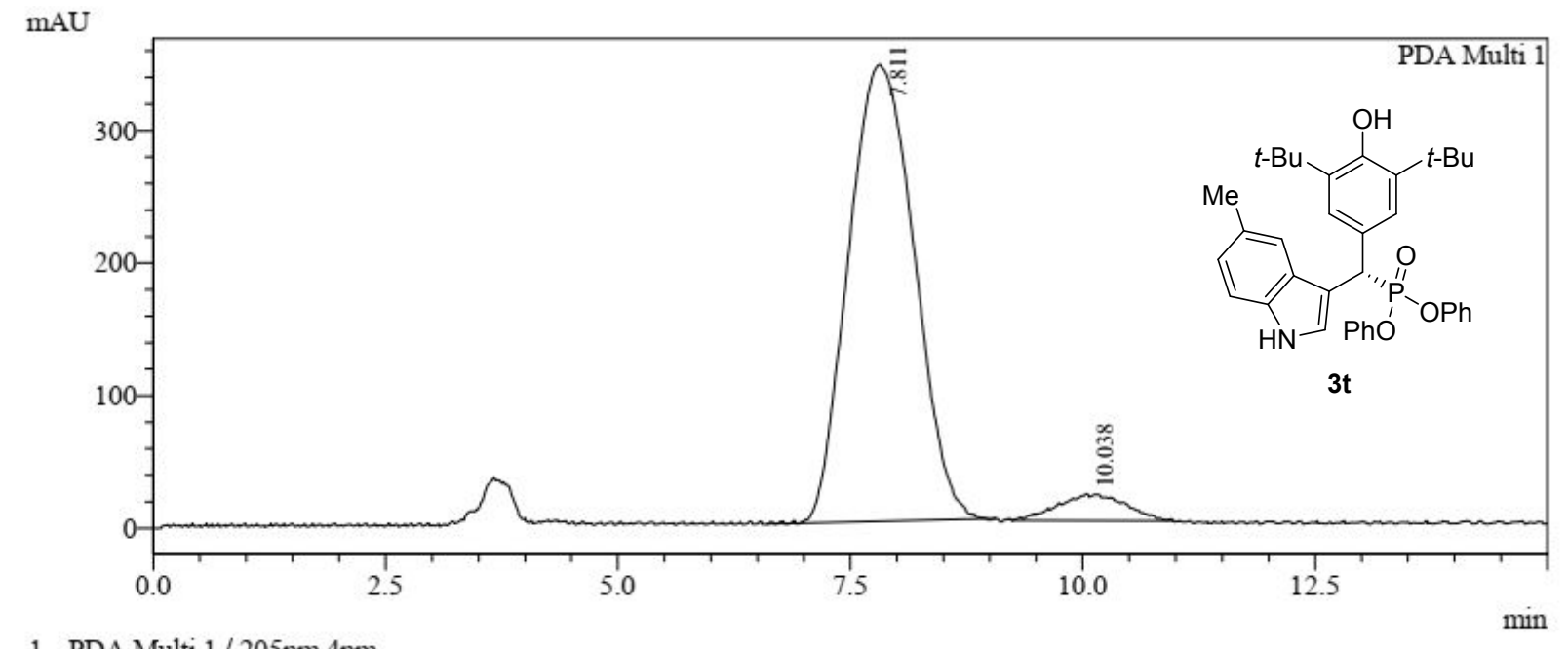

1 PDA Multi $1 / 205 \mathrm{~nm} 4 \mathrm{~nm}$

PeakTable

Ch1 205nm 4nm
\begin{tabular}{|r|r|r|r|r|}
\hline \multicolumn{1}{|c|}{ Peak\# } & Ret. Time & \multicolumn{1}{|c|}{ Area } & \multicolumn{1}{|c|}{ Height } & \multicolumn{1}{c|}{ Area \% } \\
\hline 1 & 7.811 & 16985350 & 344398 & 94.279 \\
\hline 2 & 10.038 & 1030703 & 20251 & 5.721 \\
\hline Total & & 18016053 & 364649 & 100.000 \\
\hline
\end{tabular}




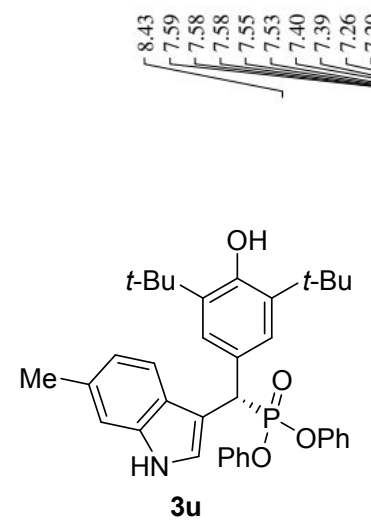

${ }^{1} \mathrm{H}-\mathrm{NMR}\left(400 \mathrm{MHz}, \mathrm{CDCl}_{3}\right)$
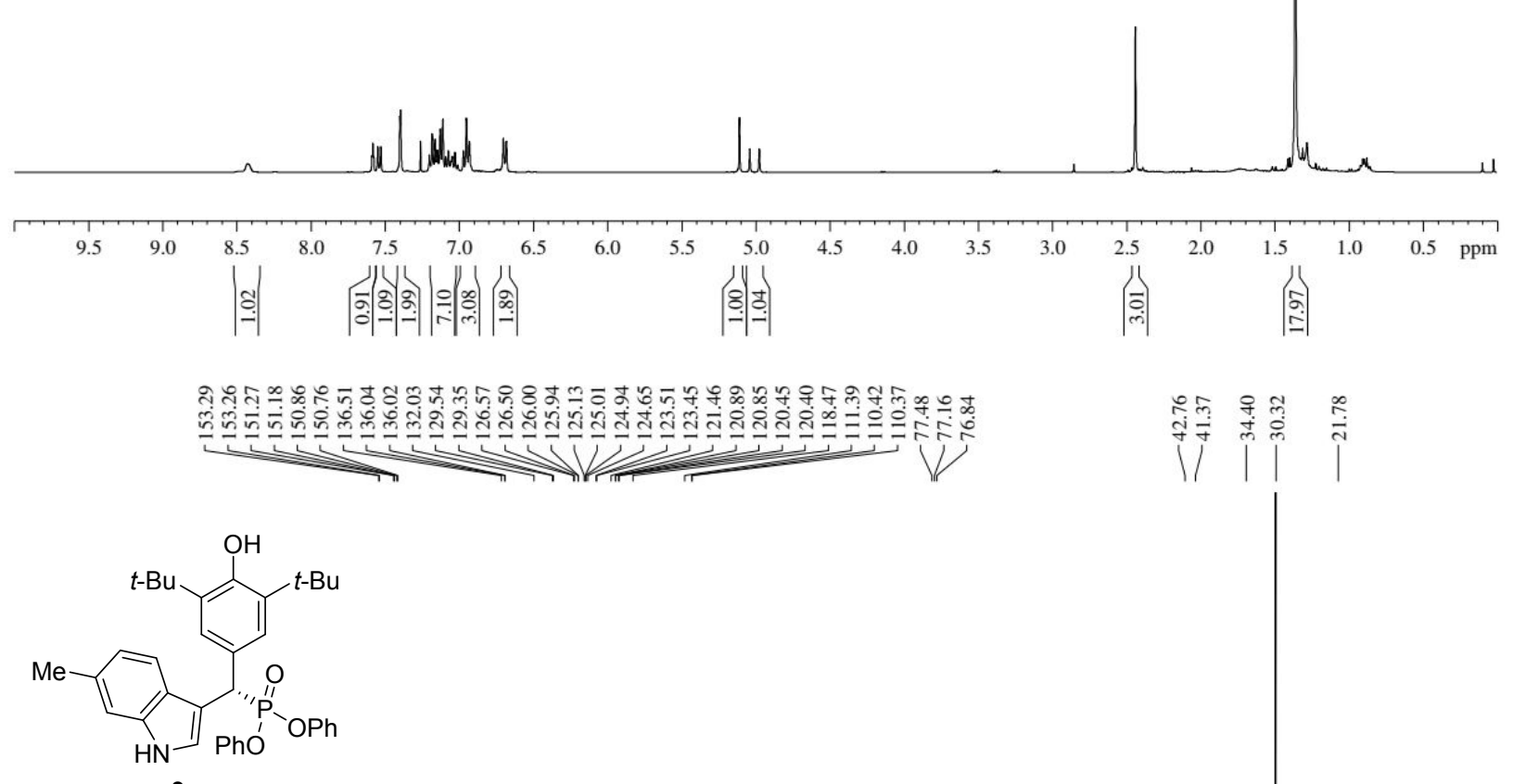

3u

${ }^{13} \mathrm{C}\left\{{ }^{1} \mathrm{H}\right\}-N M R\left(100 \mathrm{MHz}, \mathrm{CDCl}_{3}\right)$

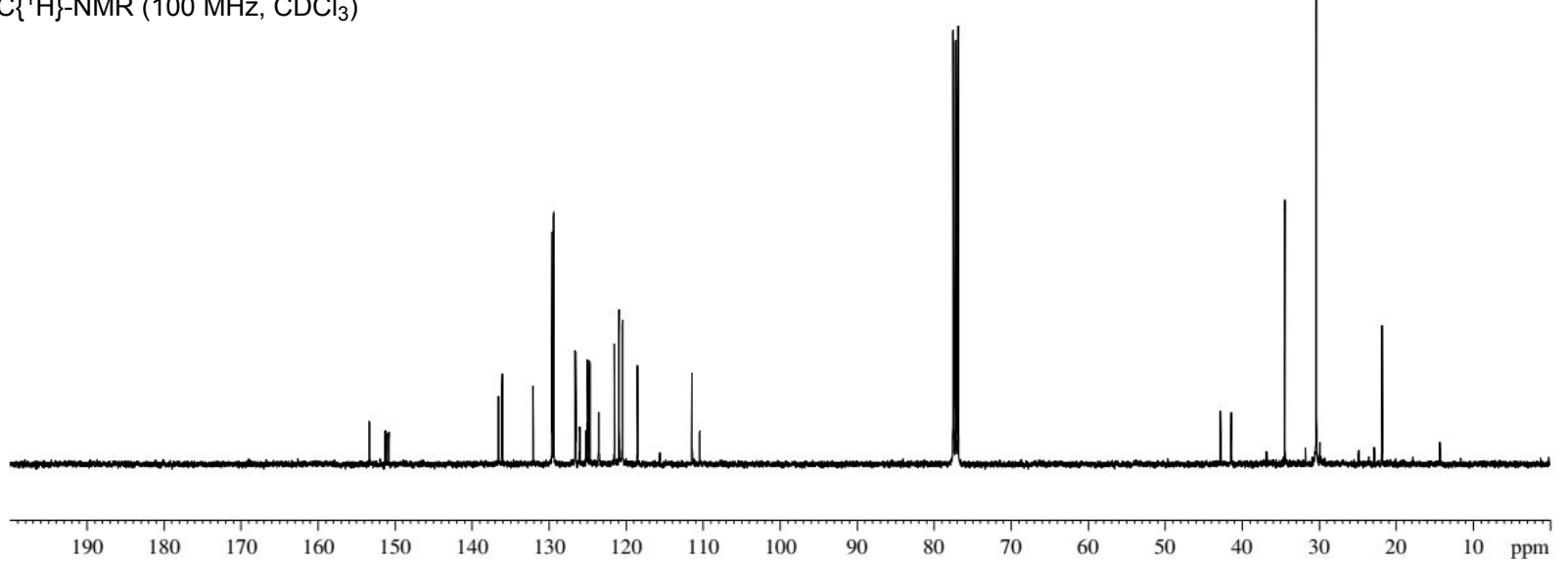




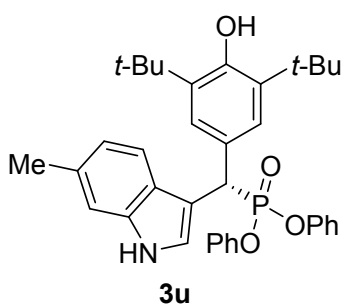

${ }^{31} \mathrm{P}-\mathrm{NMR}\left(162 \mathrm{MHz}, \mathrm{CDCl}_{3}\right)$

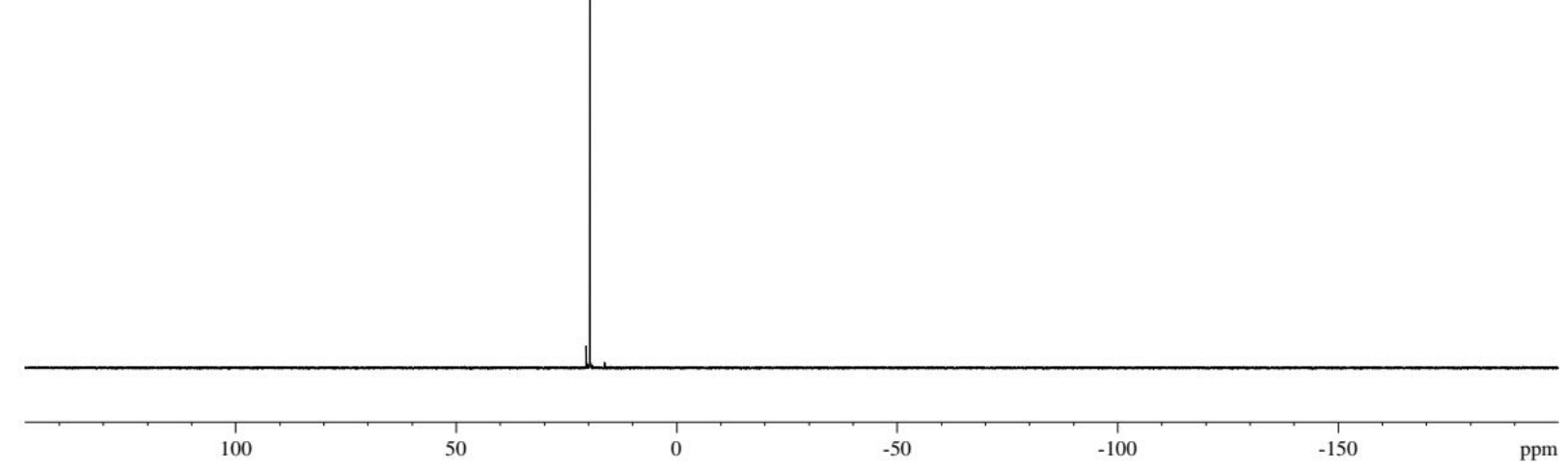

mAU

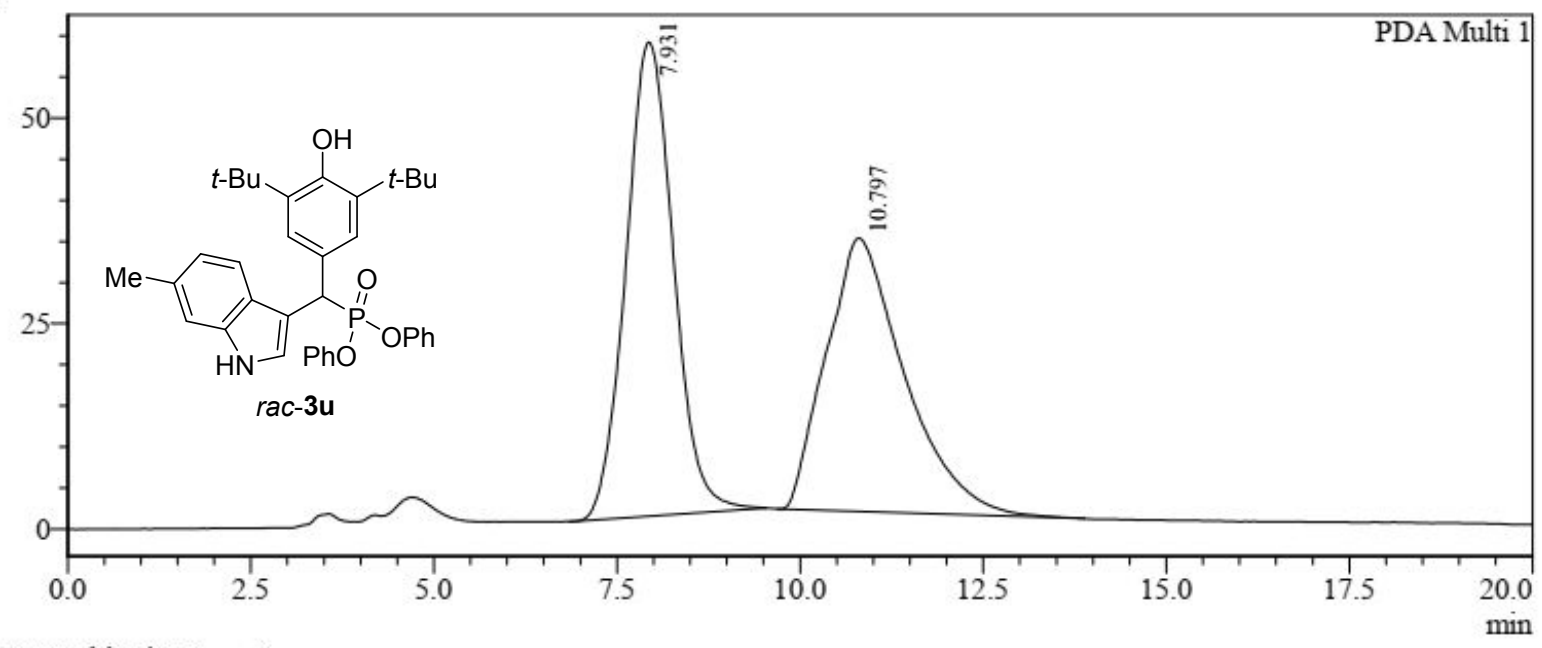

1 PDA Multi 1 / 224nm 4nm

PeakTable

Ch1 224nm 4nm
\begin{tabular}{|r|r|r|r|r|}
\multicolumn{1}{|c|}{ PeakTable } \\
\hline 1 & Ret. Time & Area & Height & \multicolumn{1}{|c|}{ Area \% } \\
\hline 2 & 7.931 & 2603042 & 57612 & 51.268 \\
\hline Total & 10.797 & 2474239 & 33219 & 48.732 \\
\hline & & 5077281 & 90832 & 100.000 \\
\hline
\end{tabular}


mAU

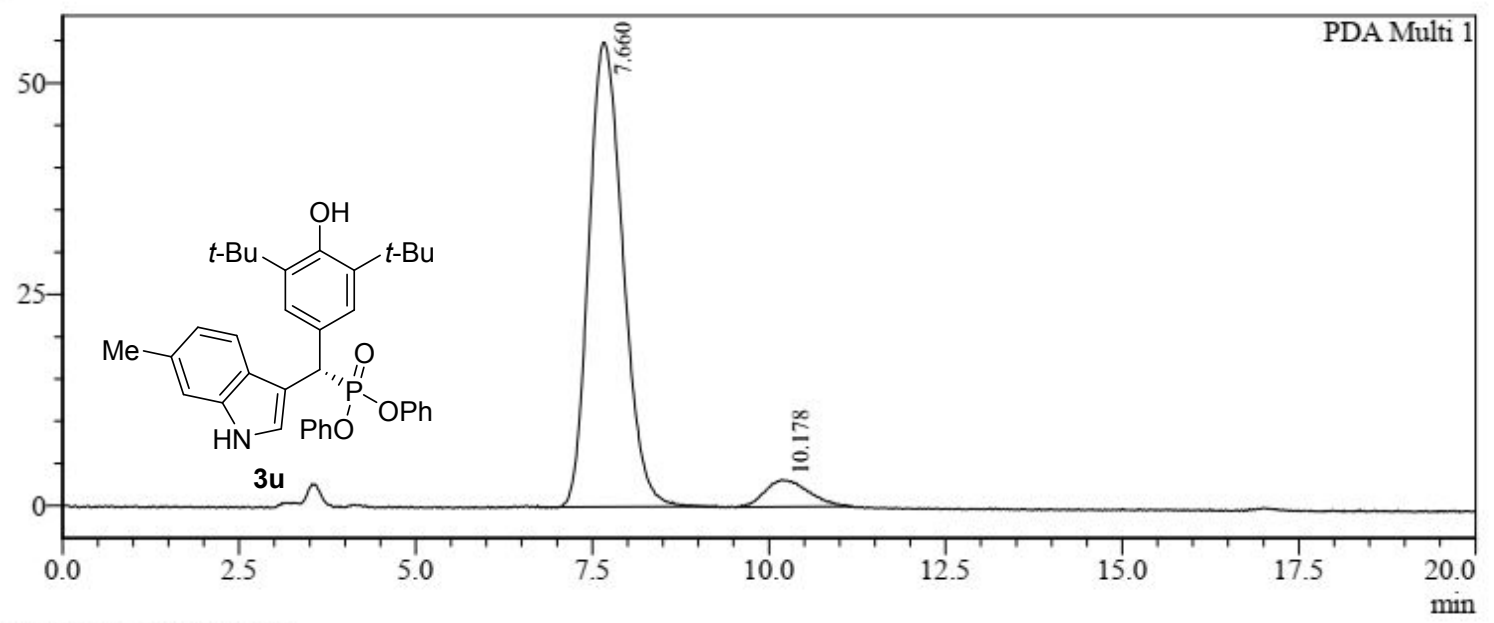

1 PDA Multi $1 / 224 \mathrm{~nm} 4 \mathrm{~nm}$

Ch1 224nm 4nm
\begin{tabular}{|r|r|r|r|r|}
\hline Peak\# & Ret. Time & \multicolumn{1}{c|}{ Area } & Height & \multicolumn{1}{c|}{ Area \% } \\
\hline 1 & 7.660 & 1843801 & 54983 & 93.069 \\
\hline 2 & 10.178 & 137303 & 3212 & 6.931 \\
\hline Total & & 1981105 & 58195 & 100.000 \\
\hline
\end{tabular}

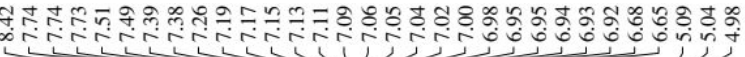

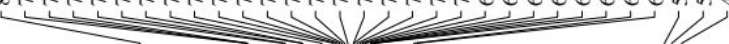<smiles>Cc1cccc2c(C(c3cc(C(C)(C)C)c(O)c(C(C)(C)C)c3)P(=O)(O)c3ccccc3)c[nH]c12</smiles>

${ }^{1} \mathrm{H}-\mathrm{NMR}\left(400 \mathrm{MHz}, \mathrm{CDCl}_{3}\right)$

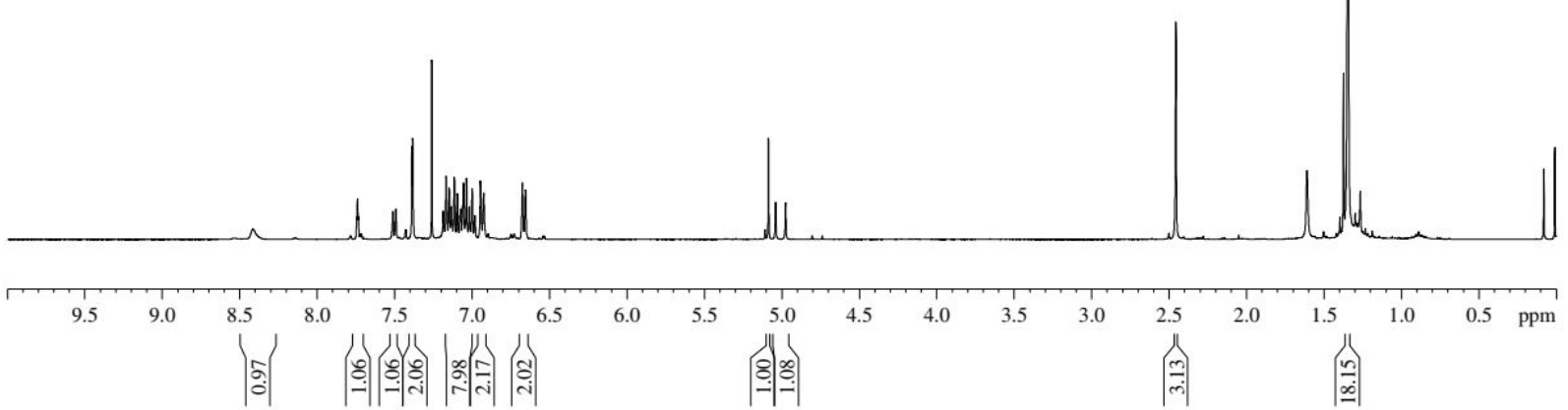




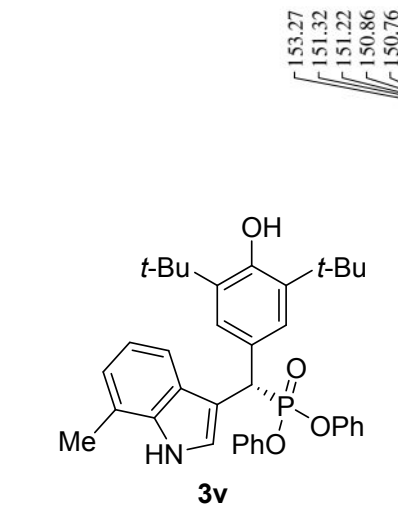

${ }^{13} \mathrm{C}\left\{{ }^{1} \mathrm{H}\right\}-\mathrm{NMR}\left(100 \mathrm{MHz}, \mathrm{CDCl}_{3}\right)$
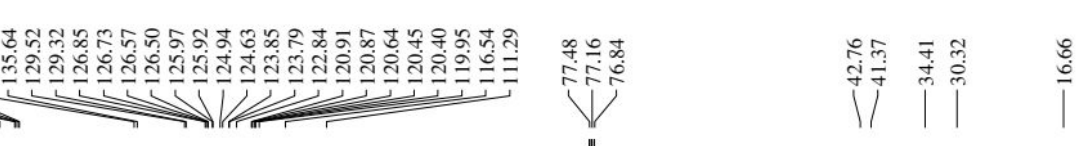

C\{
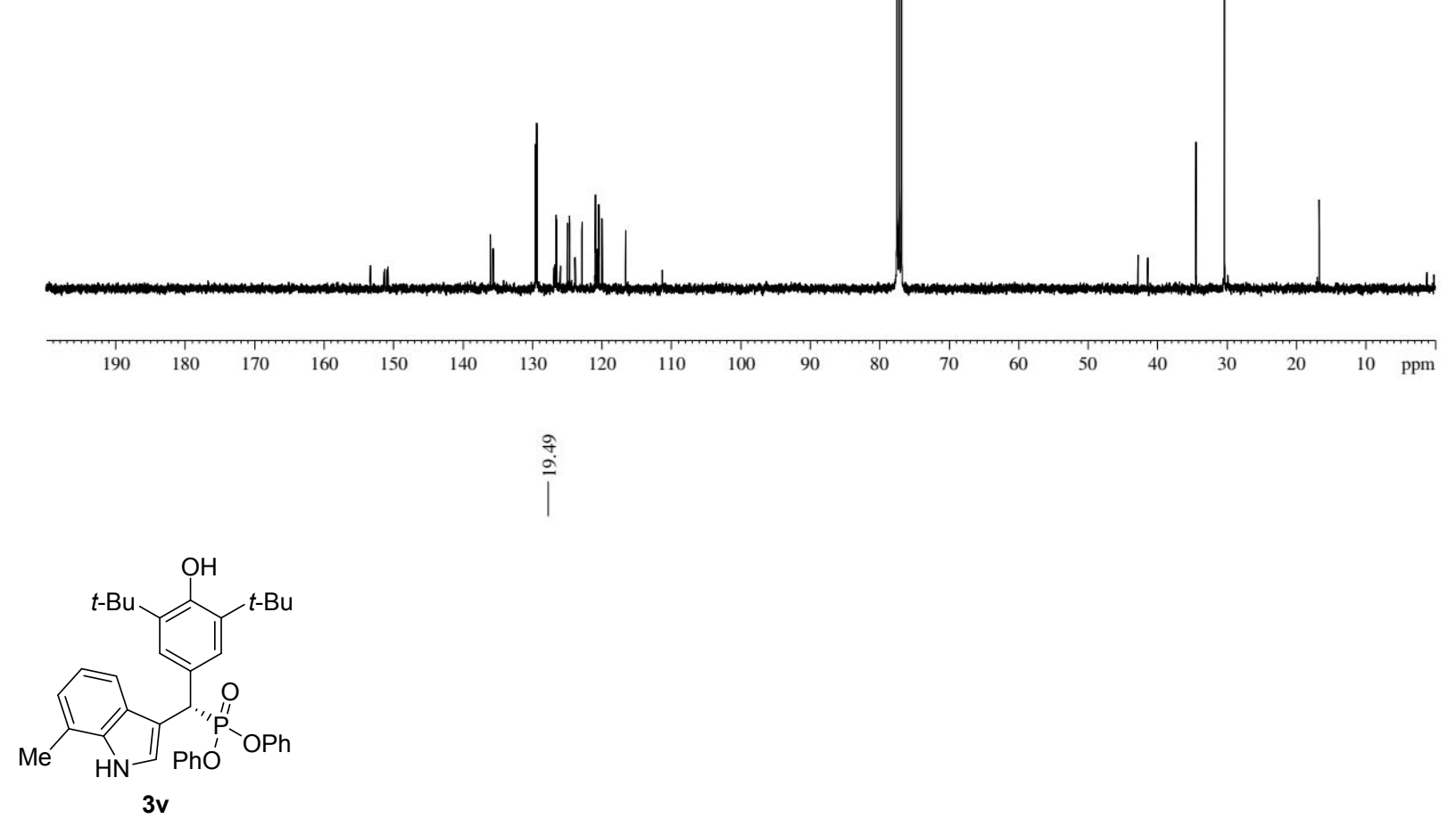

${ }^{31}$ P-NMR (162 MHz, $\mathrm{CDCl}_{3}$ )

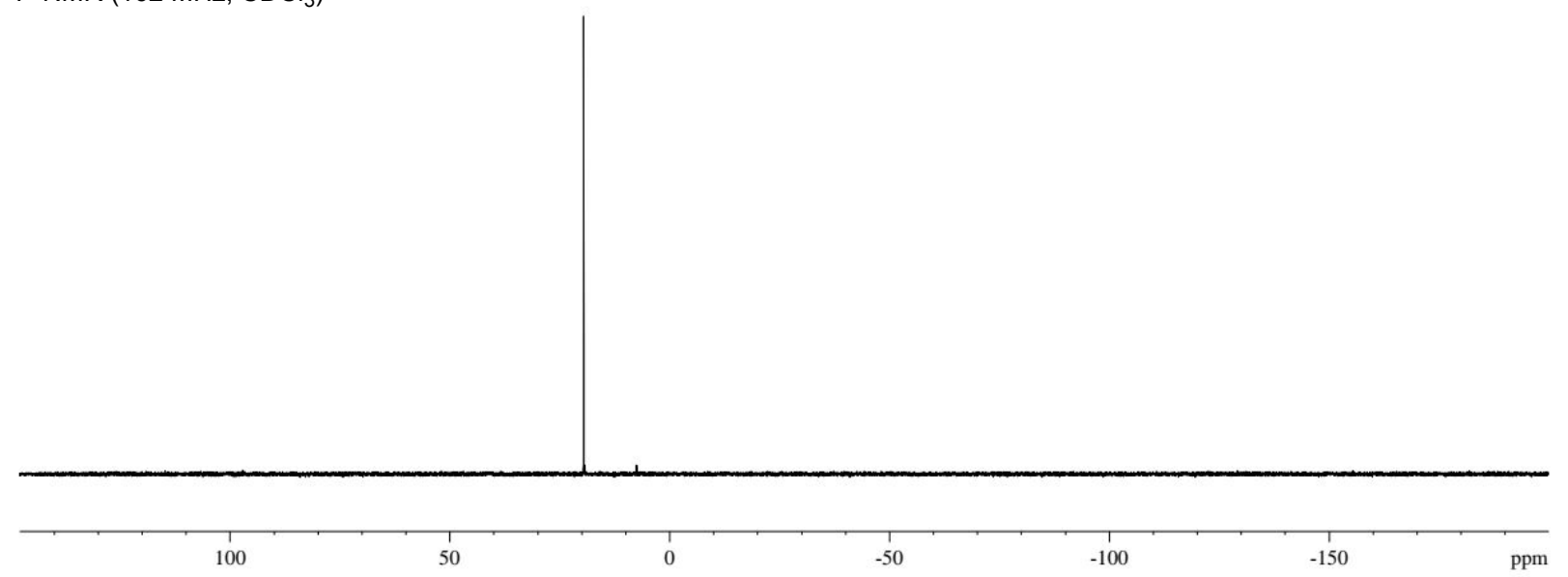


mAU

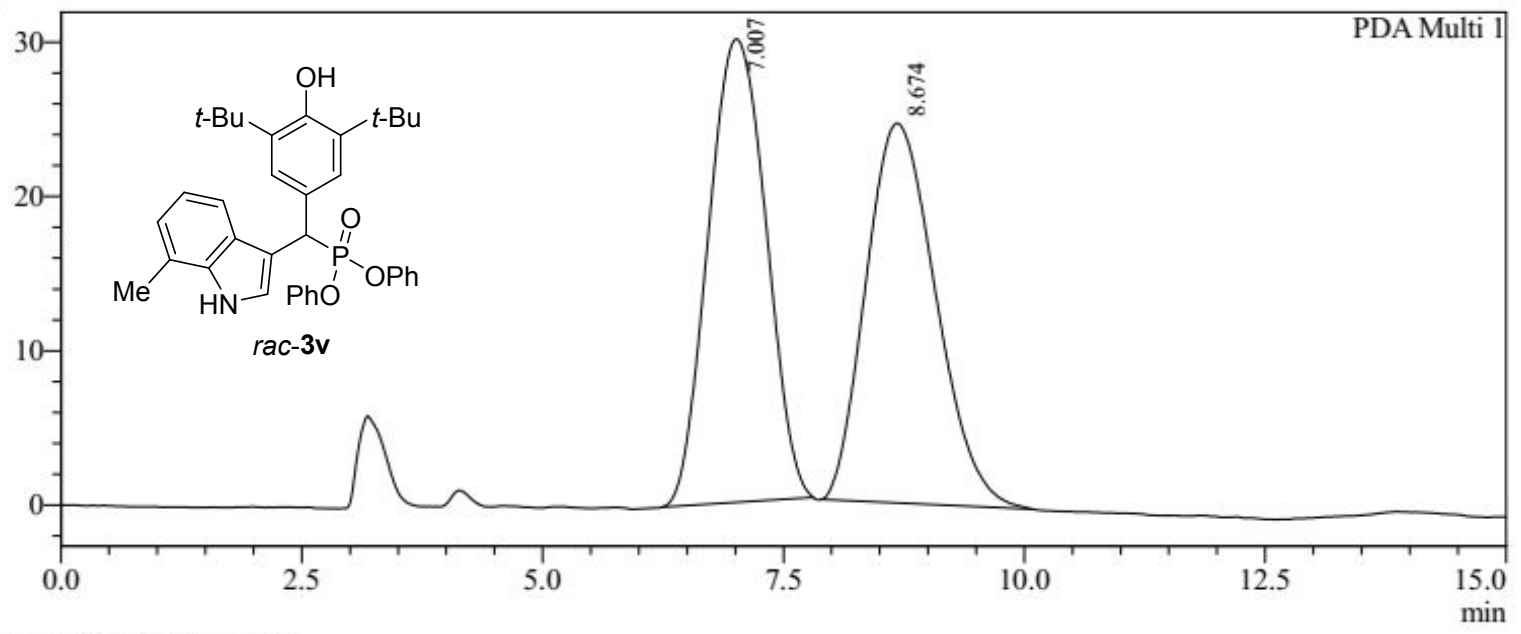

1 PDA Multi $1 / 224 \mathrm{~nm}, 4 \mathrm{~nm}$

PDA Ch1 224 nm
\begin{tabular}{|r|r|r|r|r|}
\hline \multicolumn{5}{|c|}{ PeakTable } \\
\hline Peak\# & Ret. Time & Area & Height & Area $\%$ \\
\hline 1 & 7.007 & 1246479 & 29860 & 50.220 \\
\hline 2 & 8.674 & 1235577 & 24475 & 49.780 \\
\hline Total & & 2482056 & 54335 & 100.000 \\
\hline
\end{tabular}

mAU

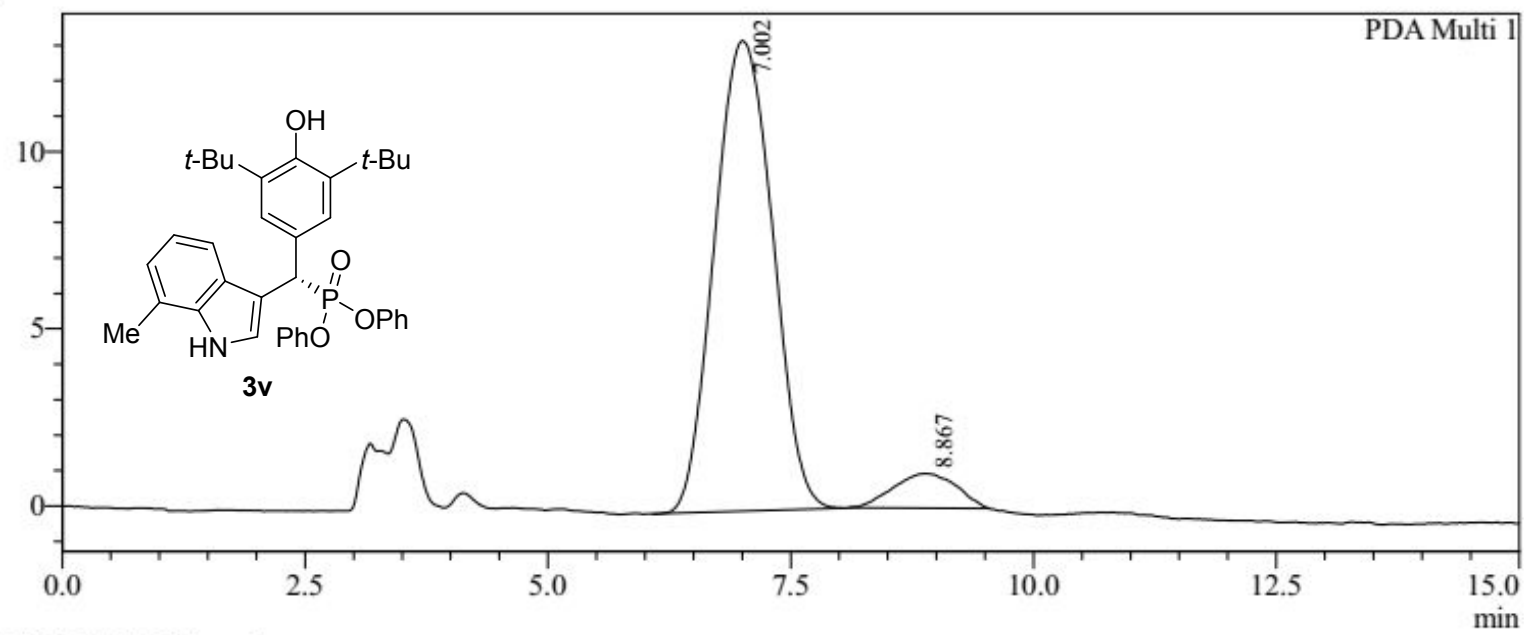

1 PDA Multi $1 / 224 \mathrm{~nm}, 4 \mathrm{~nm}$

PDA Chl $224 \mathrm{~nm}$
\begin{tabular}{|r|r|r|r|r|}
\hline \multicolumn{1}{|c|}{ Peak\# } & Ret. Time & \multicolumn{1}{c|}{ Area } & Height & \multicolumn{1}{c|}{ Area $\%$} \\
\hline 1 & 7.002 & 551796 & 13206 & 92.626 \\
\hline 2 & 8.867 & 43928 & 968 & 7.374 \\
\hline Total & & 595724 & 14174 & 100.000 \\
\hline
\end{tabular}




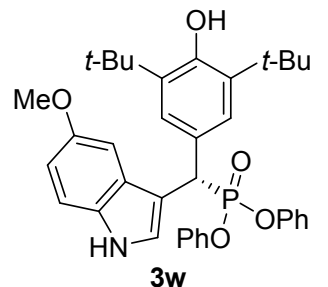

${ }^{1} \mathrm{H}-\mathrm{NMR}\left(400 \mathrm{MHz}, \mathrm{CDCl}_{3}\right)$
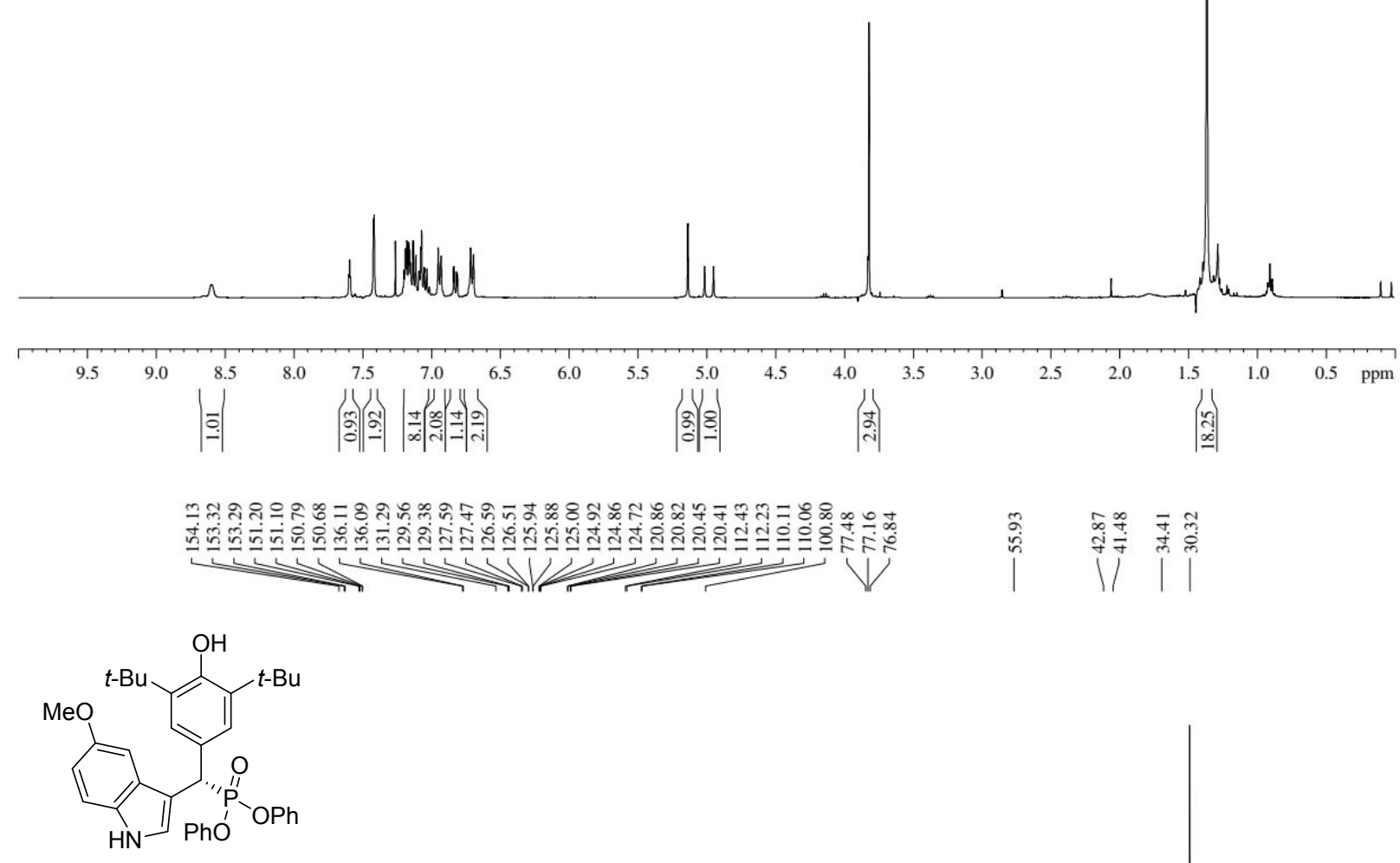

$3 w$

${ }^{13} \mathrm{C}\left\{{ }^{1} \mathrm{H}\right\}-N M R\left(100 \mathrm{MHz}, \mathrm{CDCl}_{3}\right)$

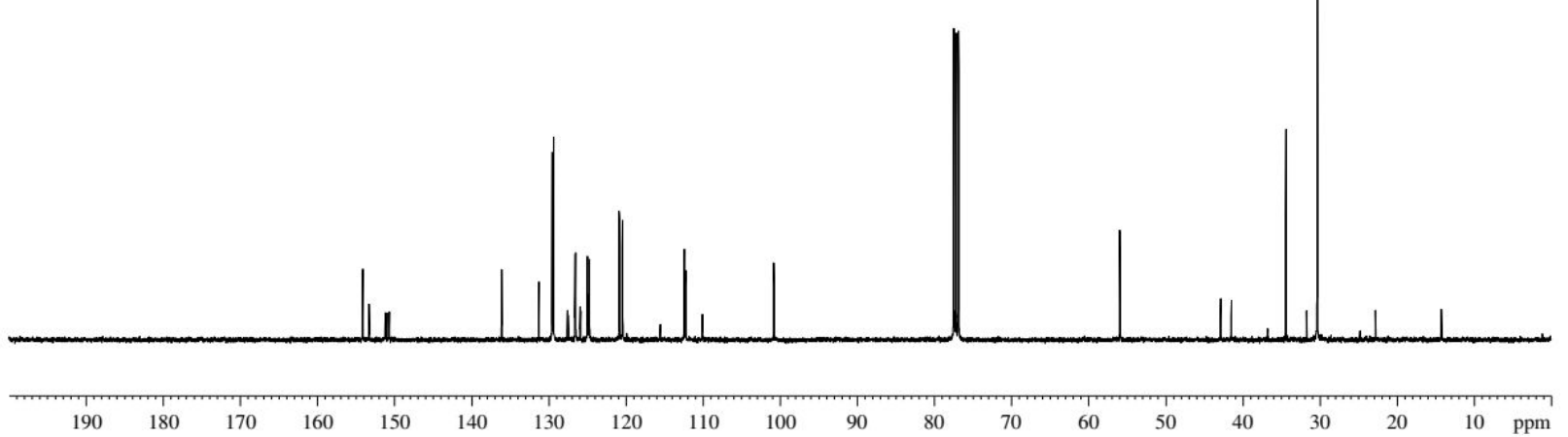


<smiles>COc1ccc2[nH]cc([C@H](c3cc(C(C)(C)C)c(O)c(C(C)(C)C)c3)P(=O)(O)Oc3ccccc3)c2c1</smiles>

${ }^{31} \mathrm{P}-\mathrm{NMR}\left(162 \mathrm{MHz}, \mathrm{CDCl}_{3}\right)$

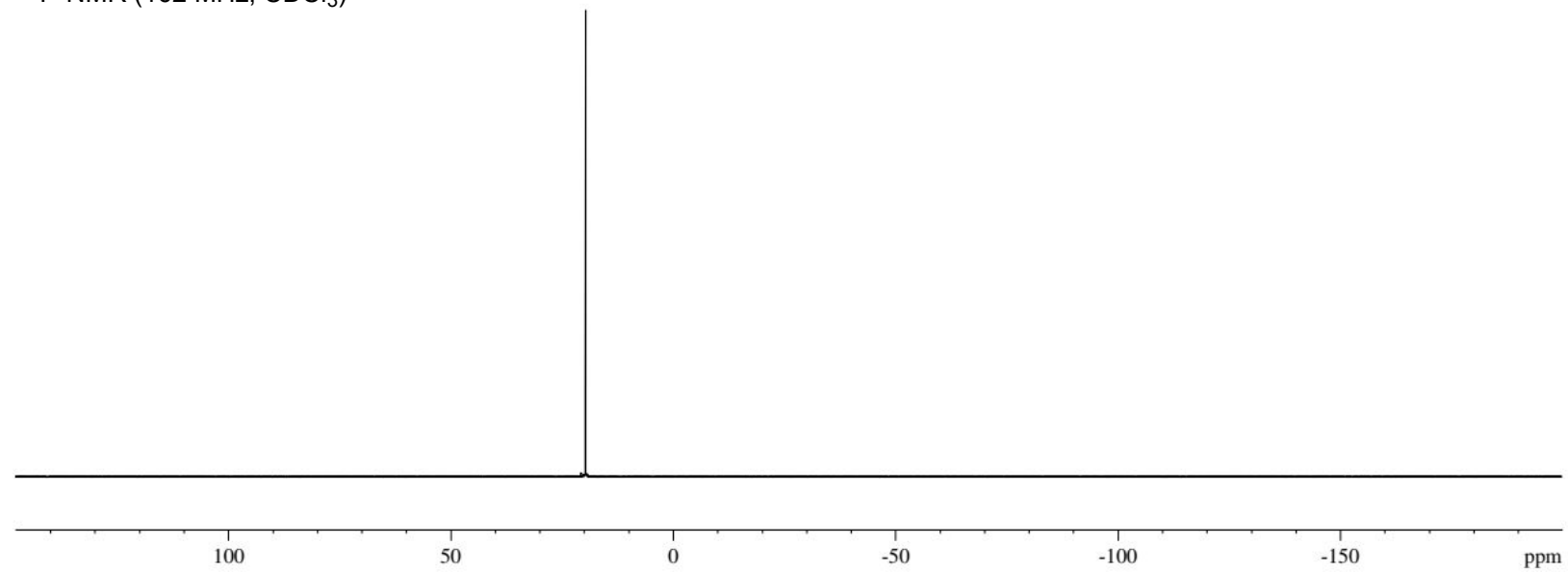

mAU

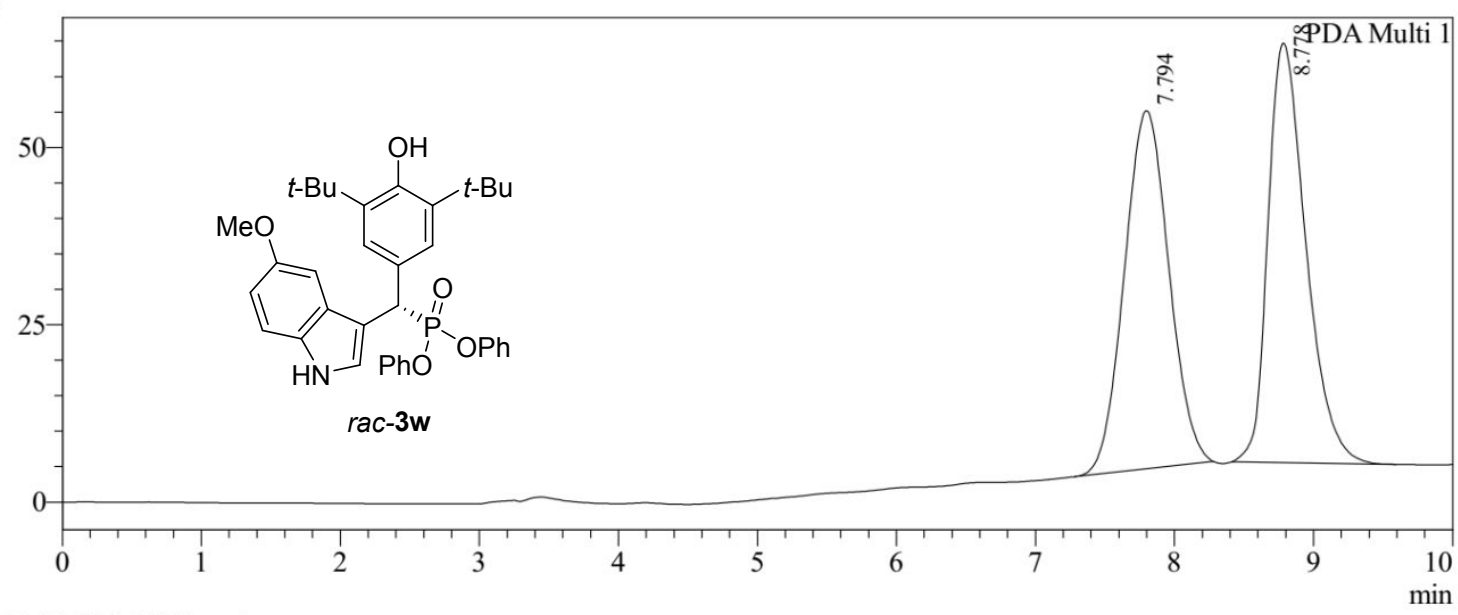

1 PDA Multi 1 / 224nm,4nm

PDA Ch1 224nm
\begin{tabular}{|r|r|r|r|r|}
\hline Peak\# & Ret. Time & Area & Height & Area $\%$ \\
\hline 1 & 7.794 & 1123496 & 50504 & 50.054 \\
\hline 2 & 8.778 & 1121065 & 59094 & 49.946 \\
\hline Total & & 2244562 & 109598 & 100.000 \\
\hline
\end{tabular}


nAU

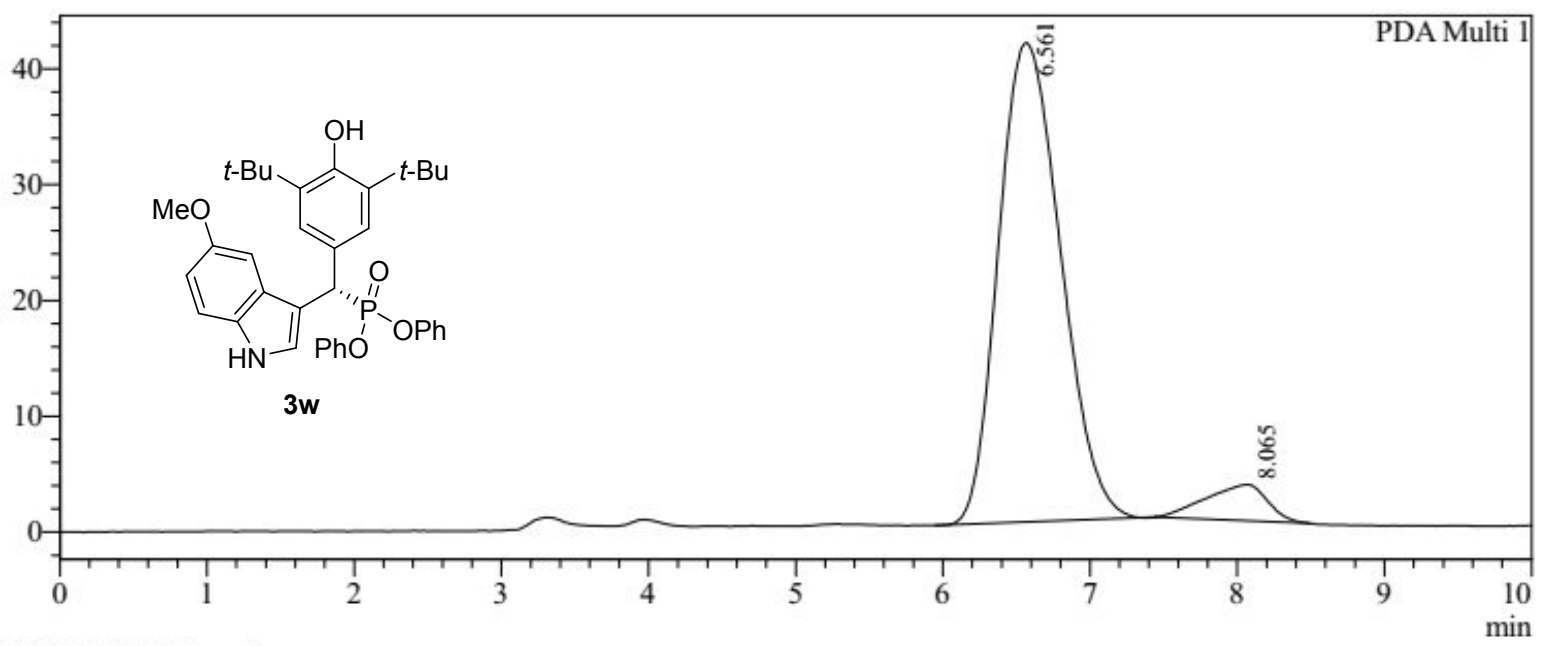

I PDA Multi $1 / 224 \mathrm{~nm}, 4 \mathrm{~nm}$

PDA Ch1 $224 \mathrm{~nm}$
\begin{tabular}{|r|r|r|r|r|}
\hline \multicolumn{1}{|c|}{ Peak\# } & Ret. Time & \multicolumn{1}{|c|}{ Area } & \multicolumn{1}{c|}{ Height } & \multicolumn{1}{|c|}{ Area $\%$} \\
\hline 1 & 6.561 & 1214095 & 41286 & 93.099 \\
\hline 2 & 8.065 & 89994 & 3106 & 6.901 \\
\hline Total & & 1304089 & 44392 & 100.000 \\
\hline
\end{tabular}
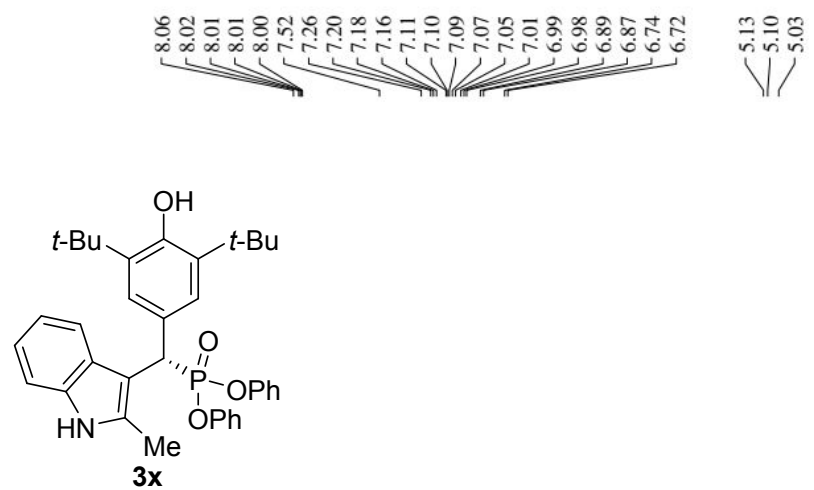

${ }^{1} \mathrm{H}-\mathrm{NMR}\left(400 \mathrm{MHz}, \mathrm{CDCl}_{3}\right)$

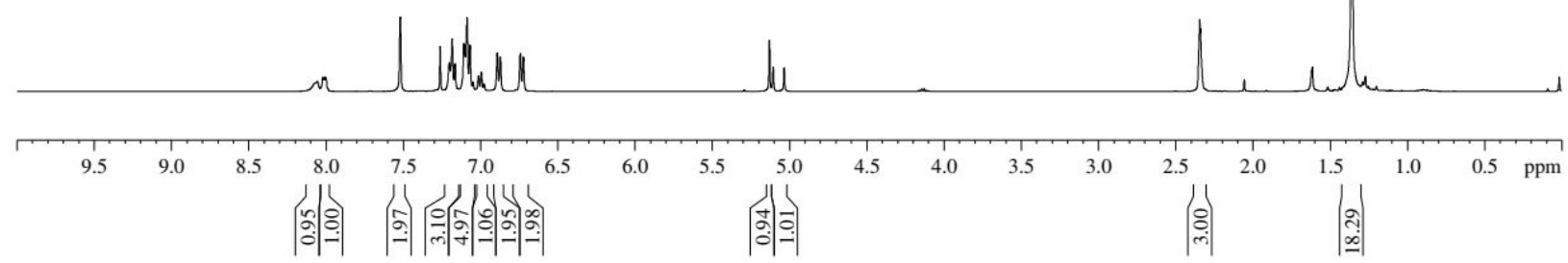



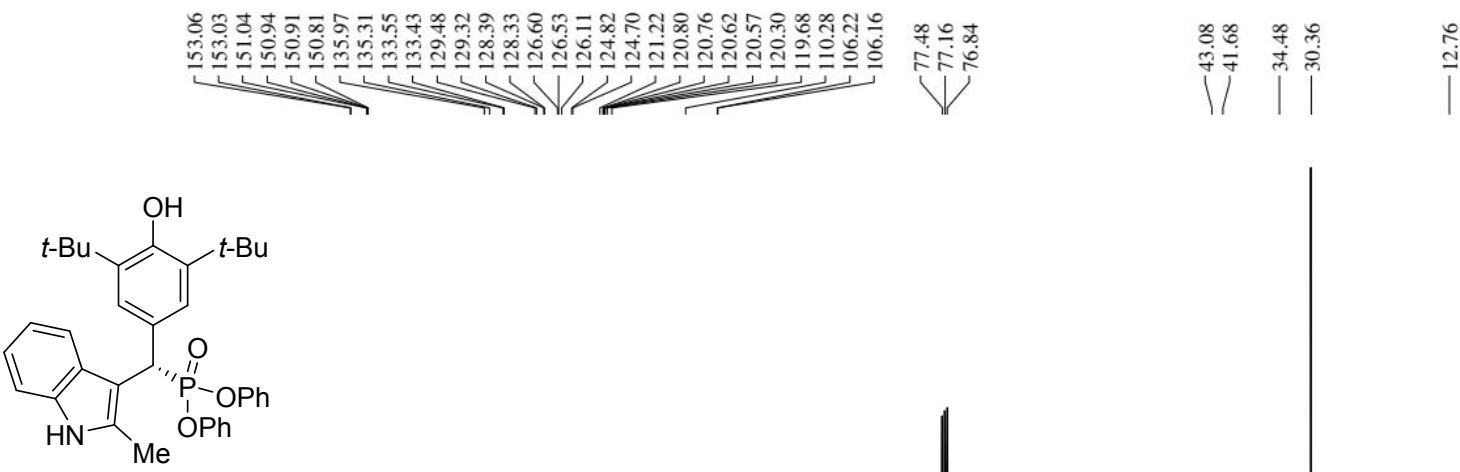

$3 \mathbf{x}$

${ }^{13} \mathrm{C}\left\{{ }^{1} \mathrm{H}\right\}-\mathrm{NMR}\left(100 \mathrm{MHz}, \mathrm{CDCl}_{3}\right)$
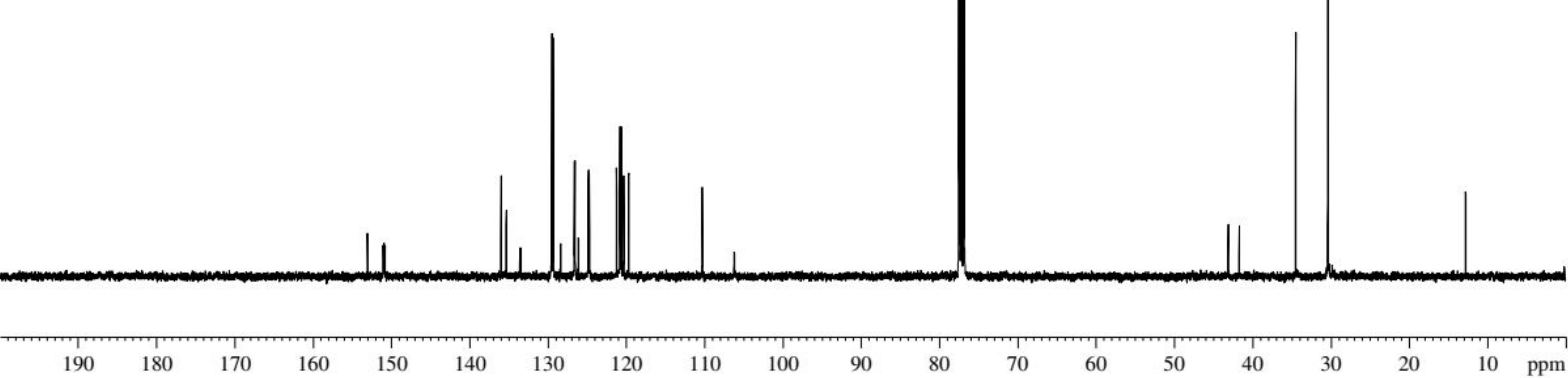

$\frac{8}{1}$

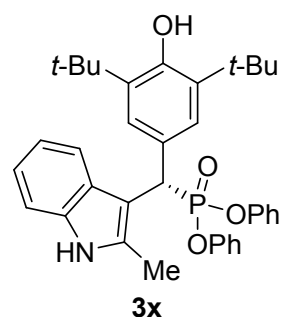

${ }^{31}$ P-NMR (162 MHz, $\mathrm{CDCl}_{3}$ ) 
mAU

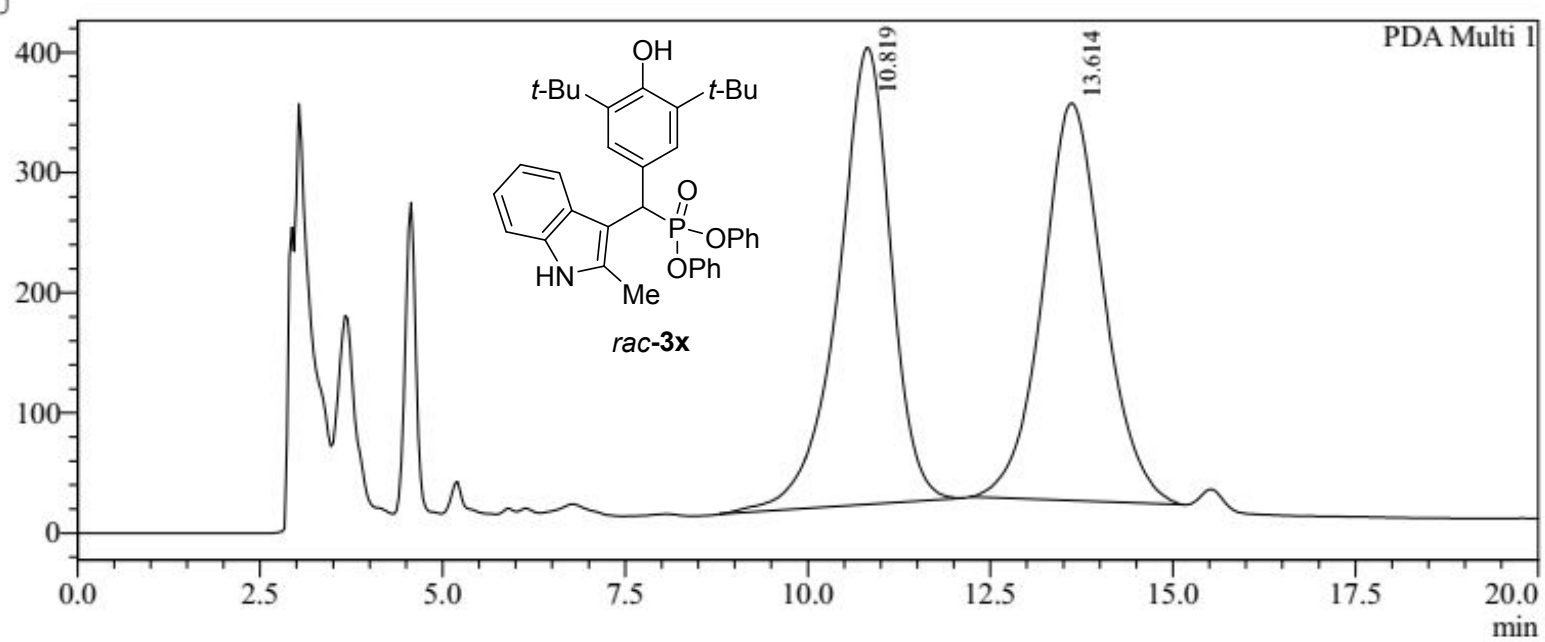

1 PDA Multi $1 / 215 \mathrm{~nm}, 4 \mathrm{~nm}$

\begin{tabular}{|c|c|c|c|c|}
\hline \multicolumn{5}{|c|}{ PDAChl $215 \mathrm{~nm}$} \\
\hline Peak\# & Ret. Time & Area & Height & Area \% \\
\hline 1 & 10.819 & 19160257 & 379921 & 49.778 \\
\hline 2 & 13.614 & 19331203 & 330644 & 50.222 \\
\hline Total & & 38491460 & 710564 & 100.000 \\
\hline
\end{tabular}

mAU

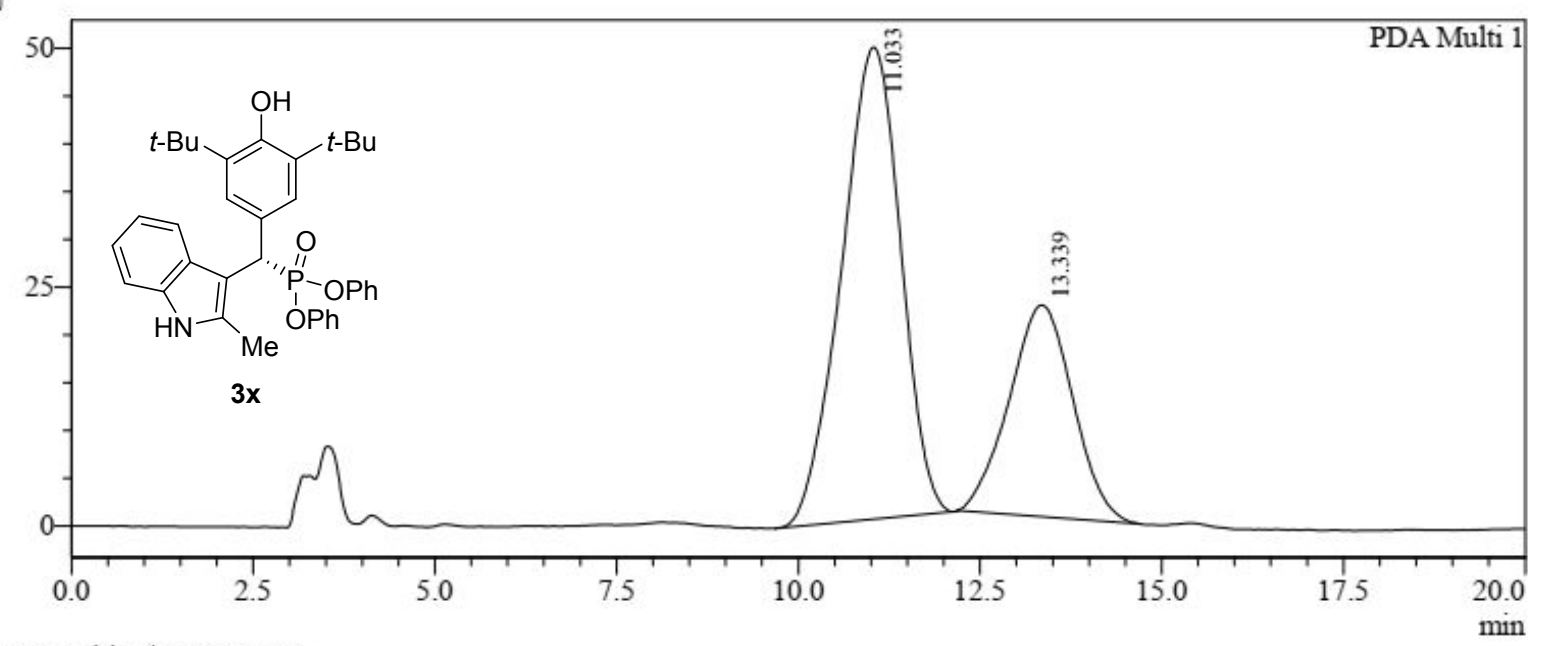

1 PDA Multi $1 / 215 \mathrm{~nm} 4 \mathrm{~nm}$

PeakTable

Ch1 $215 \mathrm{~nm} 4 \mathrm{~nm}$
\begin{tabular}{|r|r|r|r|r|}
\hline Peak\# & Ret. Time & Area & Height & \multicolumn{1}{|c|}{ Area $\%$} \\
\hline 1 & 11.033 & 2790723 & 49372 & 67.350 \\
\hline 2 & 13.339 & 1352885 & 22147 & 32.650 \\
\hline Total & & 4143608 & 71519 & 100.000 \\
\hline
\end{tabular}




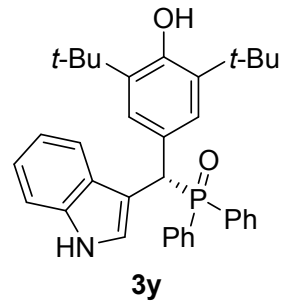

${ }^{1} \mathrm{H}-\mathrm{NMR}\left(400 \mathrm{MHz}, \mathrm{CDCl}_{3}\right)$
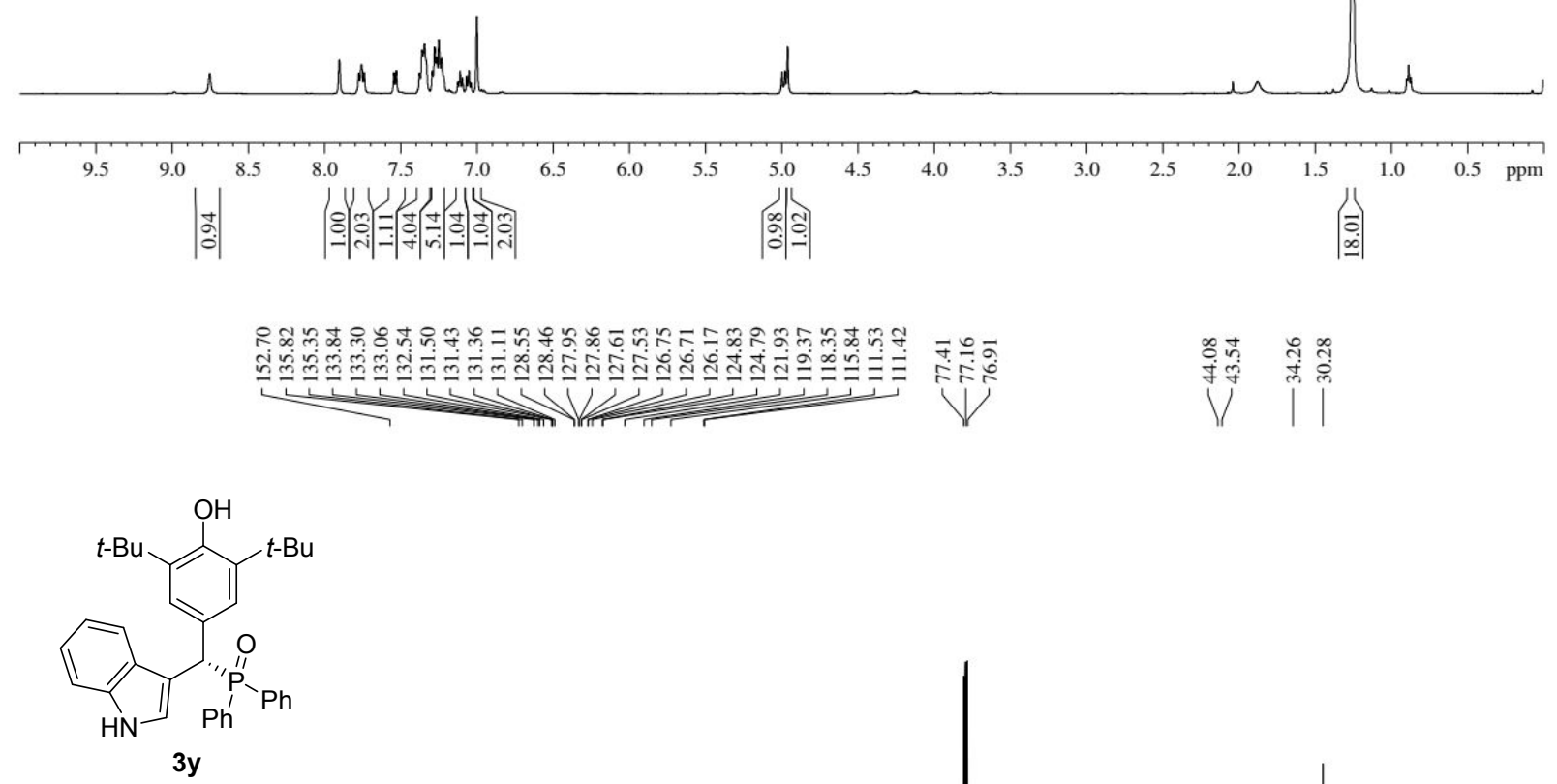

${ }^{13} \mathrm{C}\left\{{ }^{1} \mathrm{H}\right\}-\mathrm{NMR}\left(100 \mathrm{MHz}, \mathrm{CDCl}_{3}\right)$
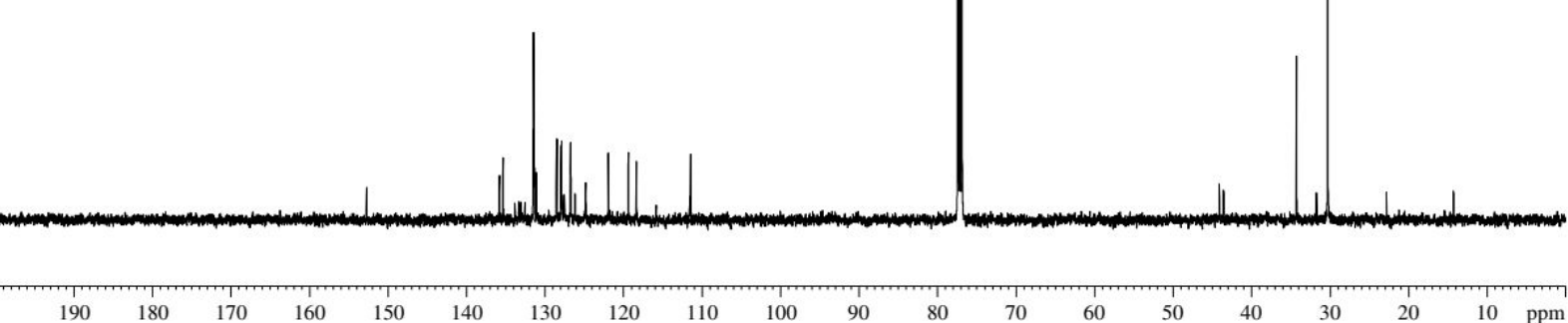


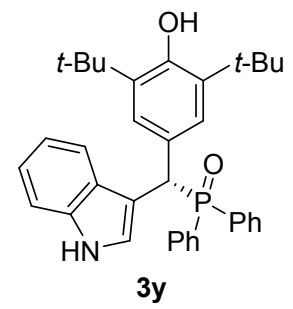

${ }^{31} \mathrm{P}-\mathrm{NMR}\left(162 \mathrm{MHz}, \mathrm{CDCl}_{3}\right)$

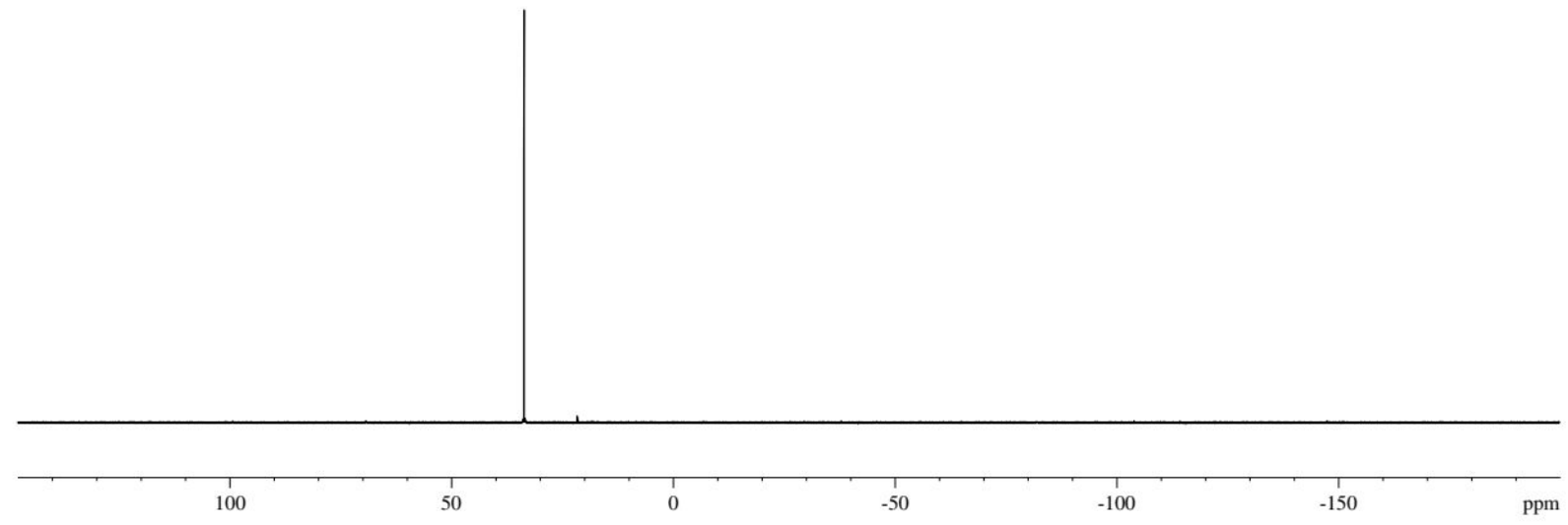

mAU

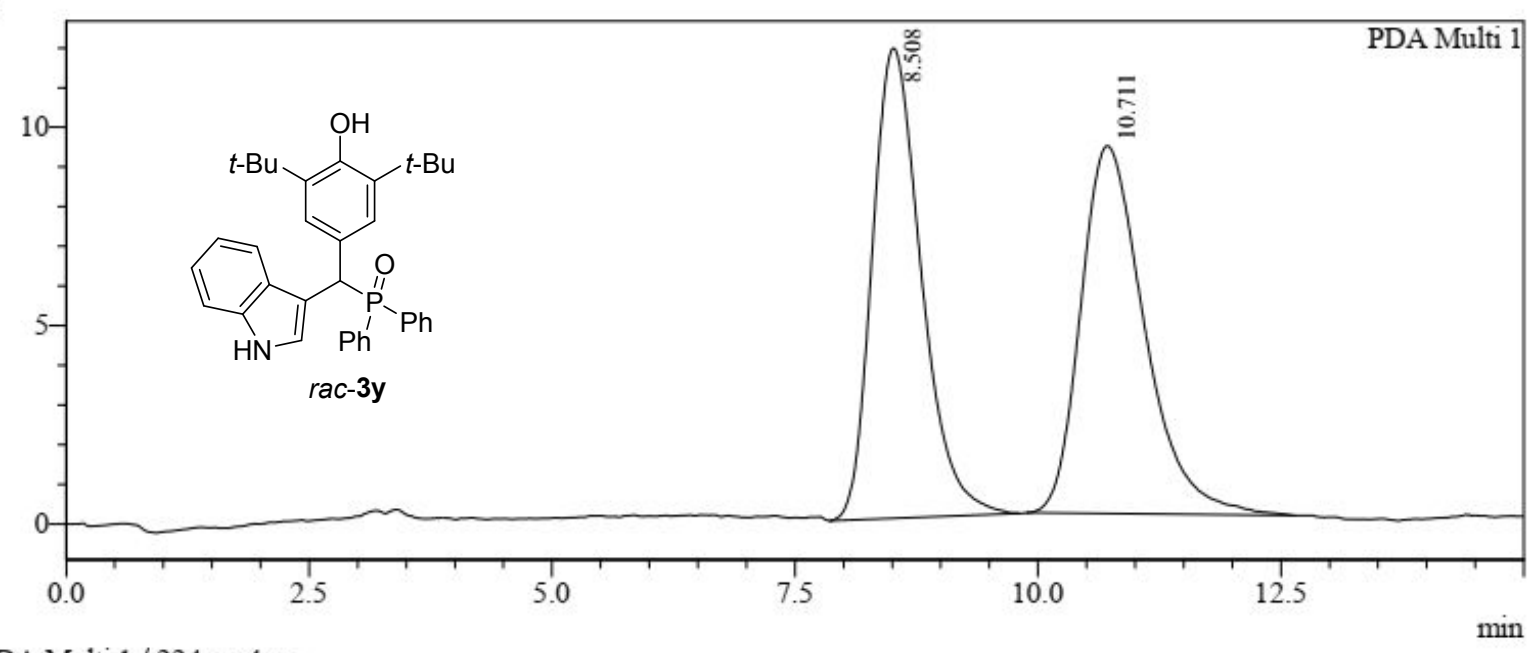

1 PDA Multi 1 / 224nm 4nm

PeakTable

Ch1 $224 \mathrm{~nm} 4 \mathrm{~nm}$
\begin{tabular}{|r|r|r|r|r|}
\hline Peak\# & Ret. Time & Area & Height & \multicolumn{1}{|c|}{ Area \% } \\
\hline 1 & 8.508 & 416818 & 11852 & 49.548 \\
\hline 2 & 10.711 & 424421 & 9273 & 50.452 \\
\hline Total & & 841239 & 21125 & 100.000 \\
\hline
\end{tabular}


mAU

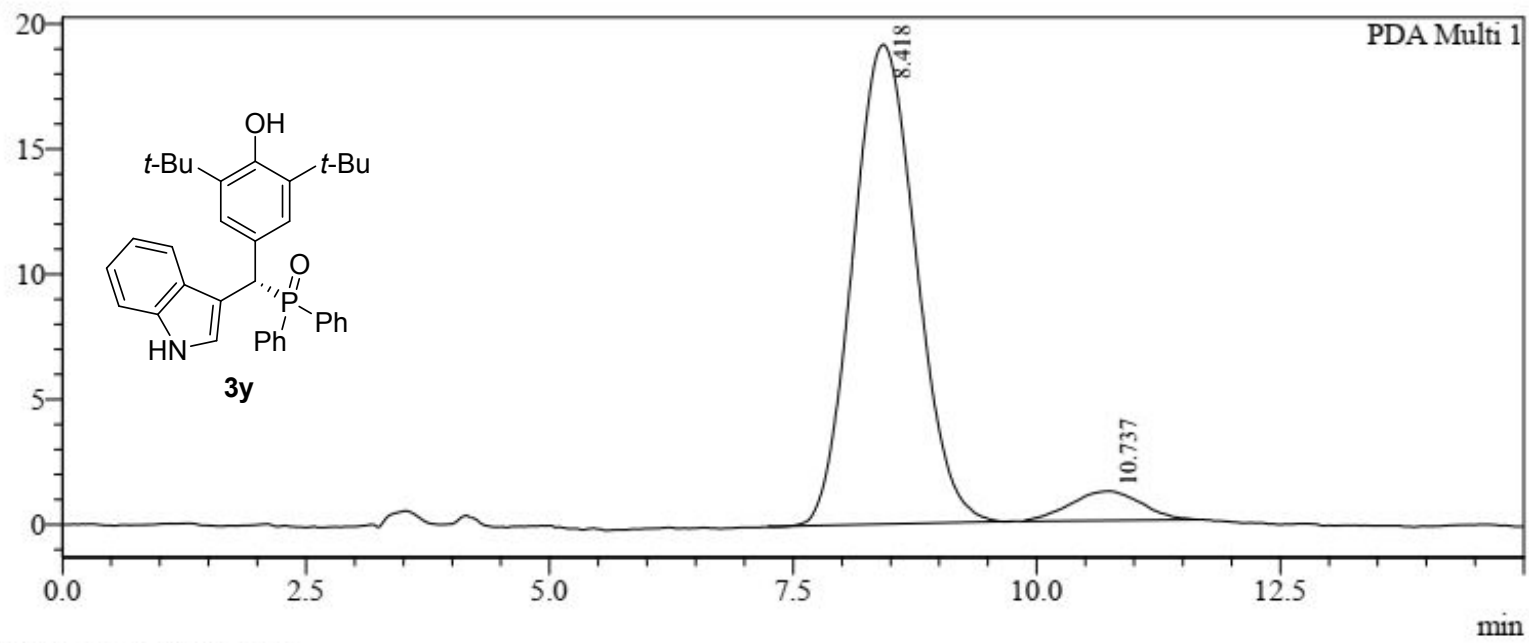

1 PDA Multi $1 / 224 \mathrm{~nm} 4 \mathrm{~nm}$

Ch1 224nm 4nm
\begin{tabular}{|r|r|r|r|r|}
\hline \multicolumn{1}{|c|}{ Peak\#\# } & Ret. Time & \multicolumn{1}{|c|}{ Area } & Height & \multicolumn{1}{c|}{ Area \% } \\
\hline 1 & 8.418 & 866730 & 19163 & 93.669 \\
\hline 2 & 10.737 & 58582 & 1166 & 6.331 \\
\hline Total & & 925312 & 20329 & 100.000 \\
\hline
\end{tabular}

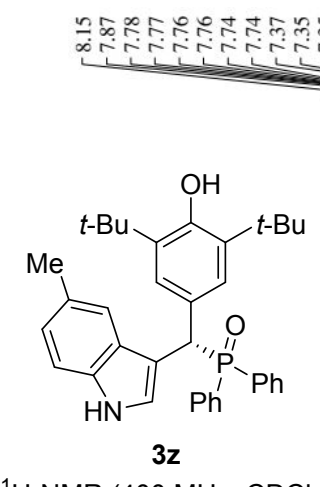

${ }^{1} \mathrm{H}-\mathrm{NMR}\left(400 \mathrm{MHz}, \mathrm{CDCl}_{3}\right)$

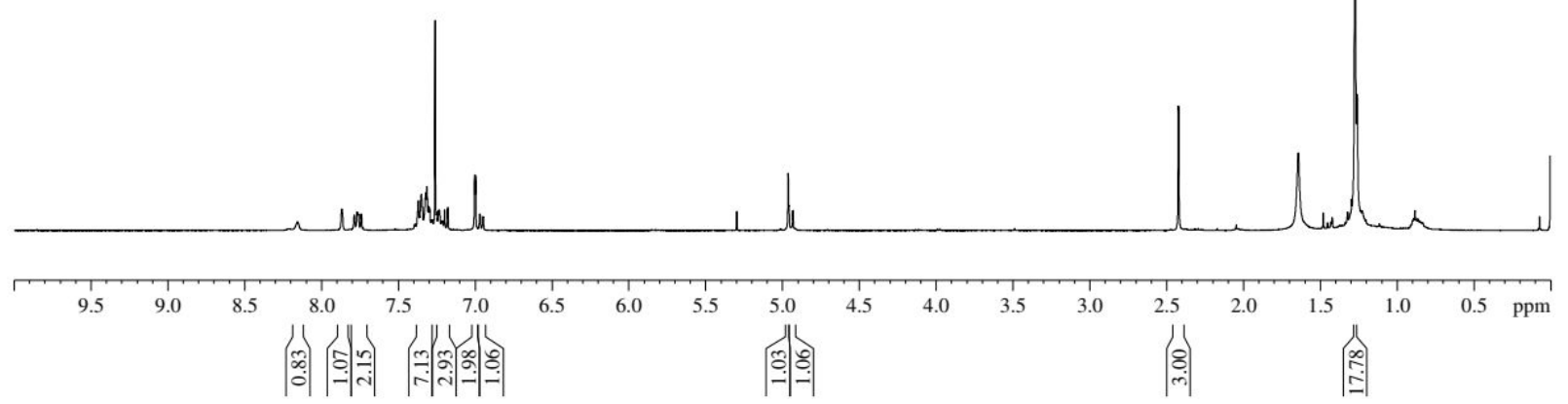



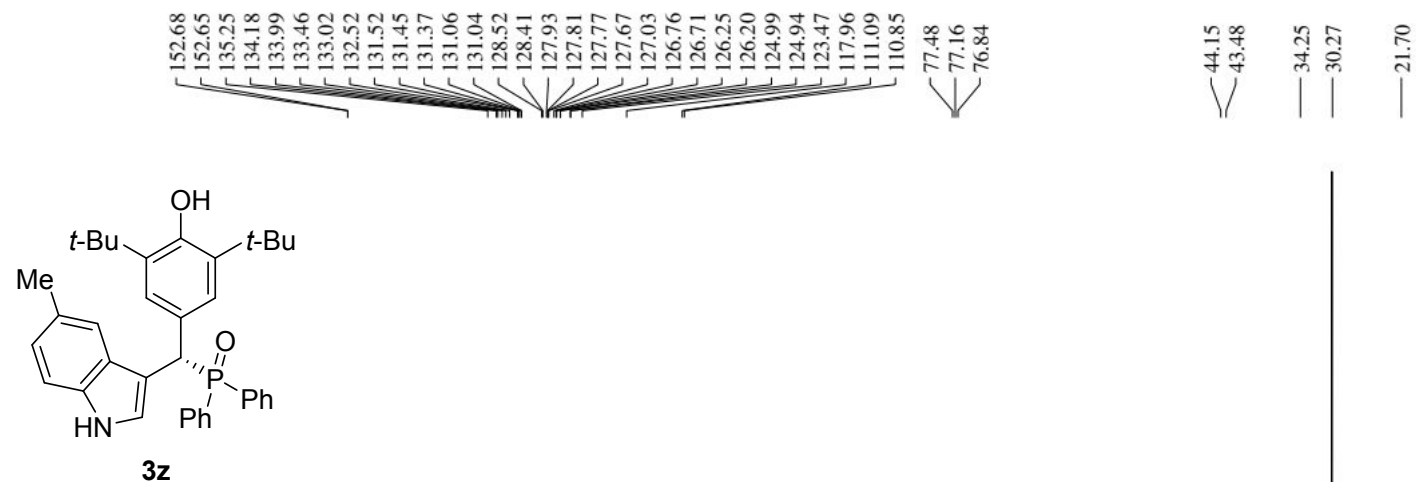

${ }^{13} \mathrm{C}\left\{{ }^{1} \mathrm{H}\right\}-N M R\left(100 \mathrm{MHz}, \mathrm{CDCl}_{3}\right)$
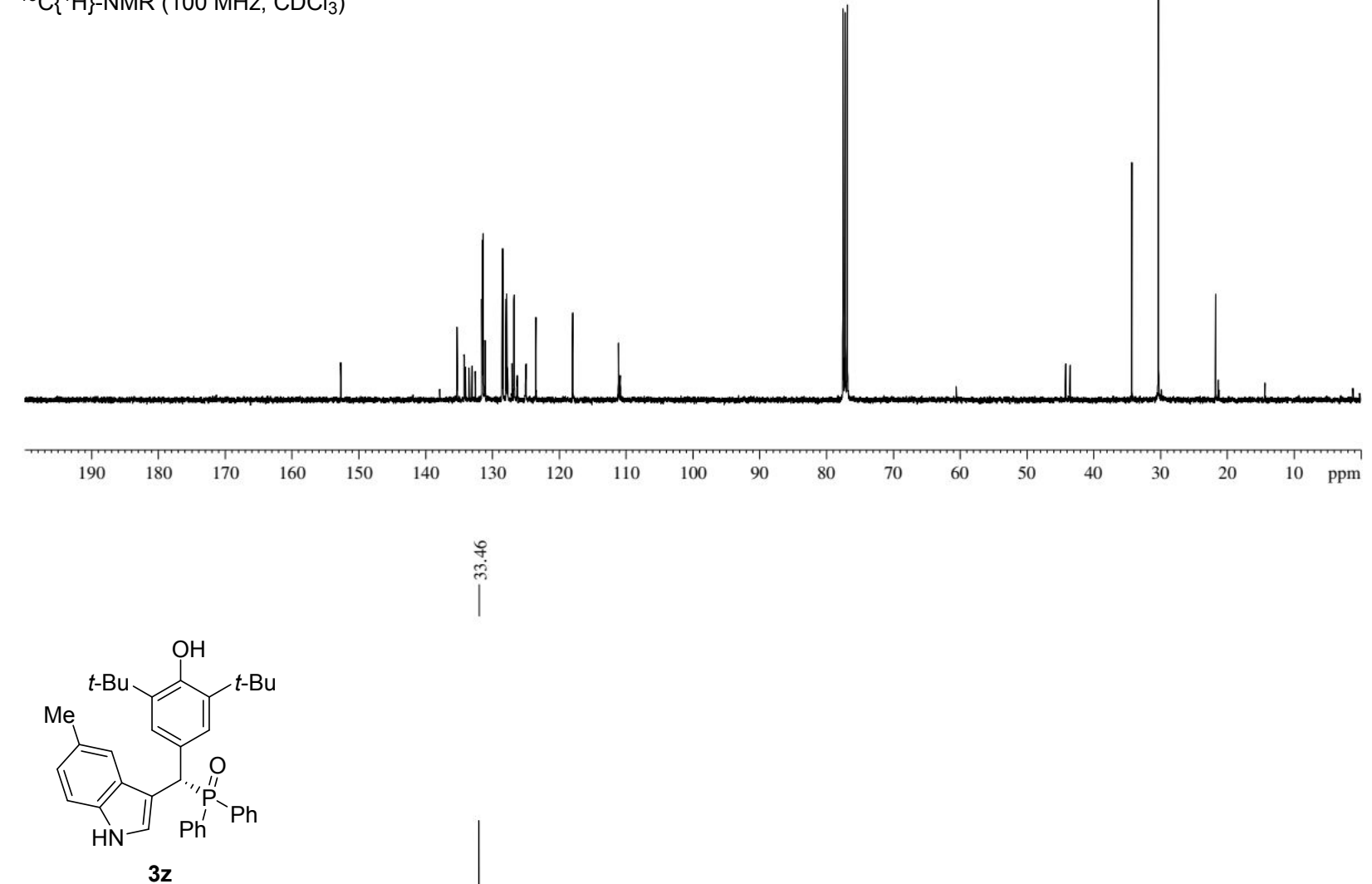

${ }^{31} \mathrm{P}-\mathrm{NMR}\left(162 \mathrm{MHz}, \mathrm{CDCl}_{3}\right.$ )

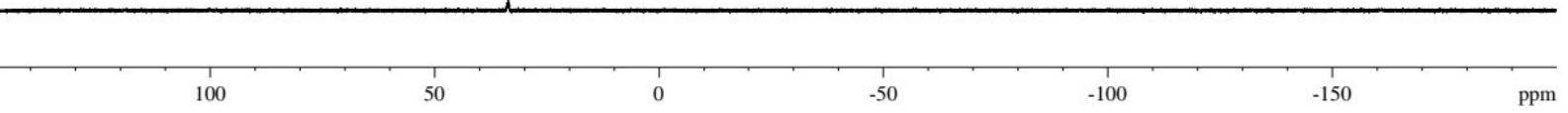


mAU

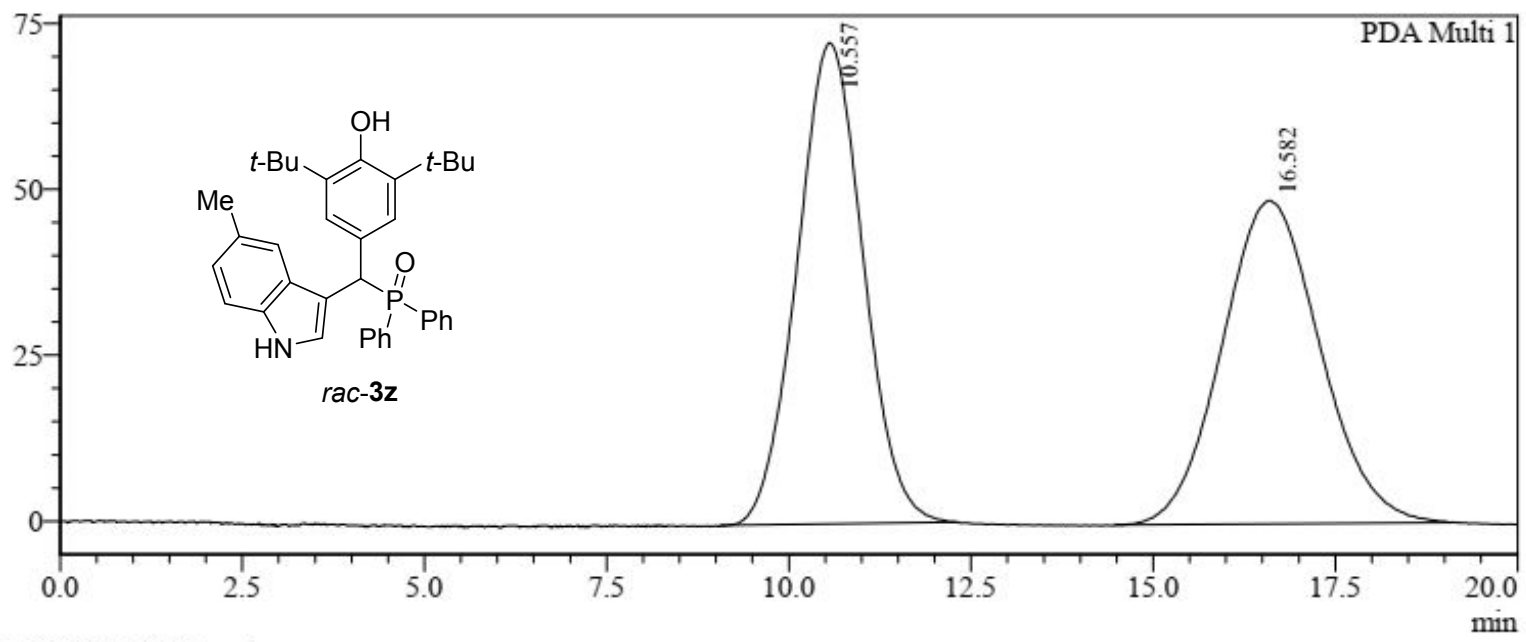

1 PDA Multi $1 / 220 \mathrm{~nm} 4 \mathrm{~nm}$

\begin{tabular}{|c|c|c|c|c|}
\hline \multirow{2}{*}{\multicolumn{5}{|c|}{$\mathrm{Ch} 1220 \mathrm{~nm} 4 \mathrm{~nm}$}} \\
\hline & & & & \\
\hline Peak\# & Ret. Time & Area & Height & Area \% \\
\hline 1 & 10.557 & 4573785 & 72491 & 50.046 \\
\hline 2 & 16.582 & 4565452 & 48719 & 49.954 \\
\hline Total & & 9139237 & 121210 & 100.000 \\
\hline
\end{tabular}

mAU

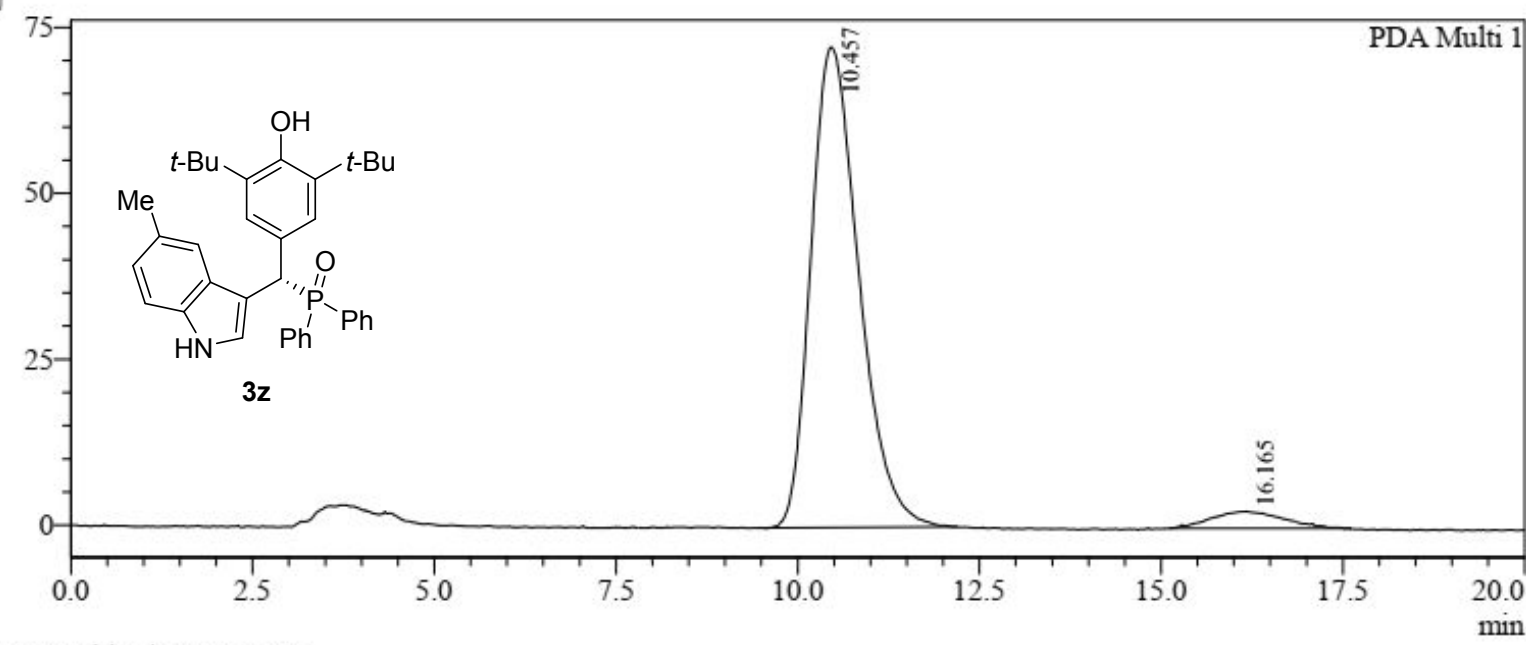

1 PDA Multi $1 / 220 \mathrm{~nm} 4 \mathrm{~nm}$

PeakTable

Ch1 220nm 4nm
\begin{tabular}{|r|r|r|r|r|}
\hline \multicolumn{1}{|c|}{ Peak\# } & Ret. Time & \multicolumn{1}{|c|}{ Area } & Height & Area \% \\
\hline 1 & 10.457 & 3316912 & 72328 & 94.887 \\
\hline 2 & 16.165 & 178717 & 2572 & 5.113 \\
\hline Total & & 3495629 & 74900 & 100.000 \\
\hline
\end{tabular}



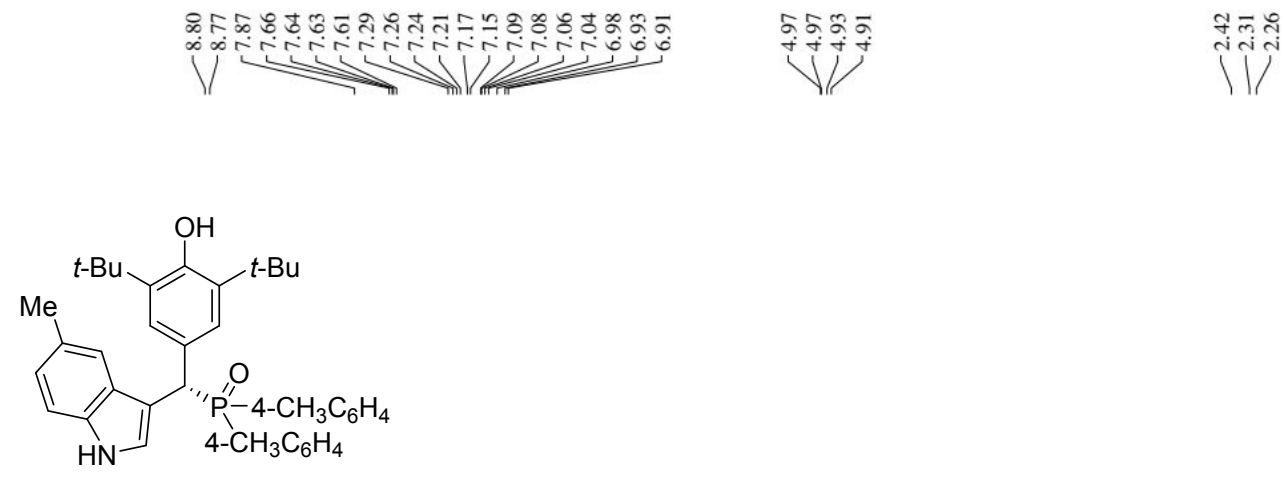

$3 z a$

${ }^{1} \mathrm{H}-\mathrm{NMR}\left(400 \mathrm{MHz}, \mathrm{CDCl}_{3}\right)$
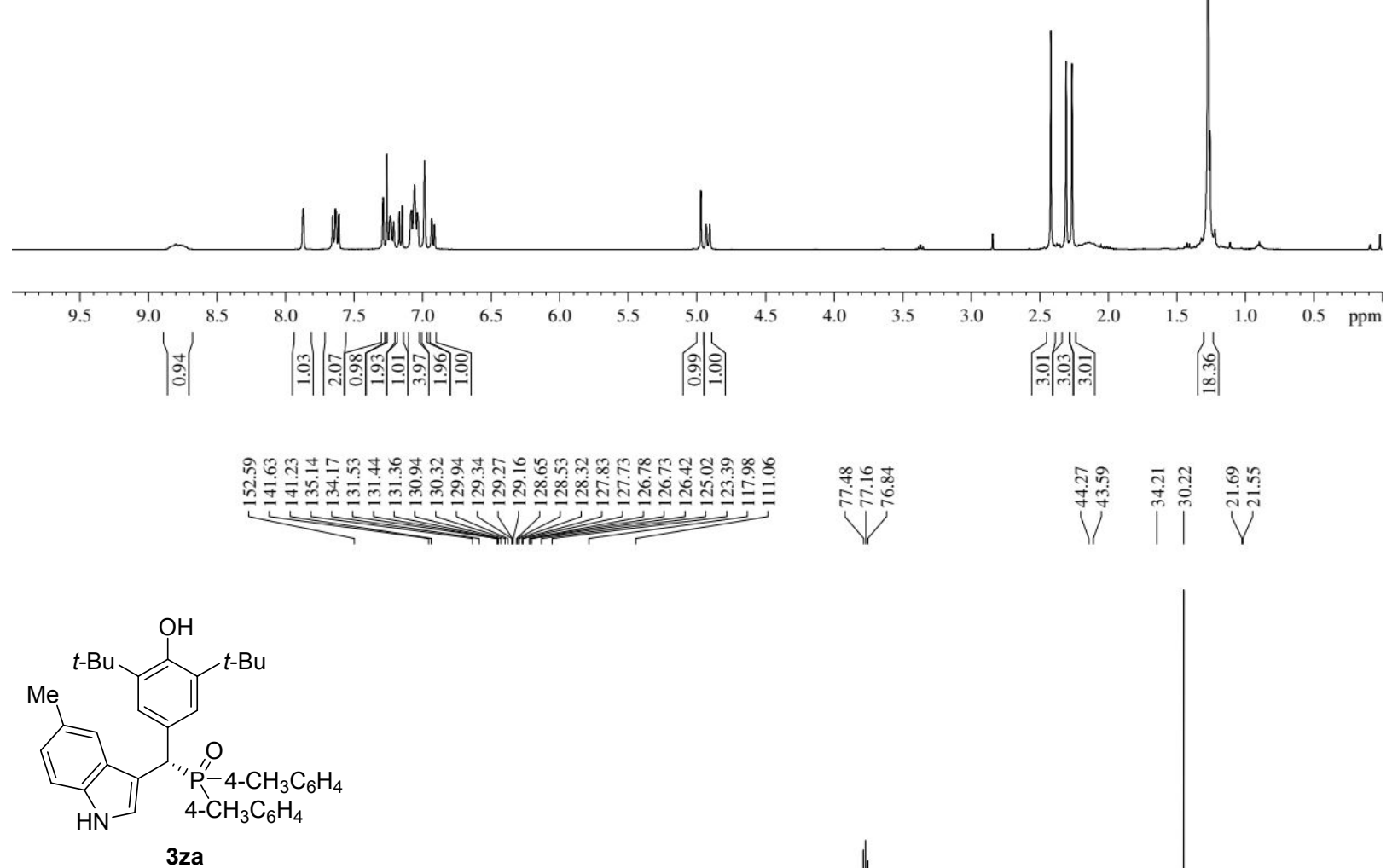

${ }^{13} \mathrm{C}\left\{{ }^{1} \mathrm{H}\right\}-\mathrm{NMR}\left(100 \mathrm{MHz}, \mathrm{CDCl}_{3}\right)$

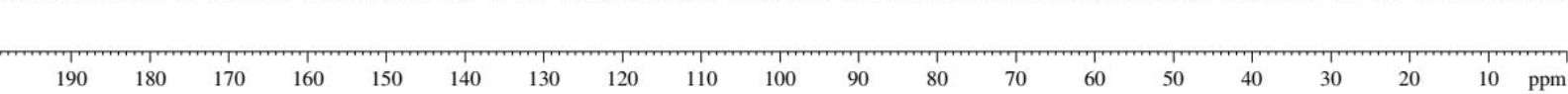



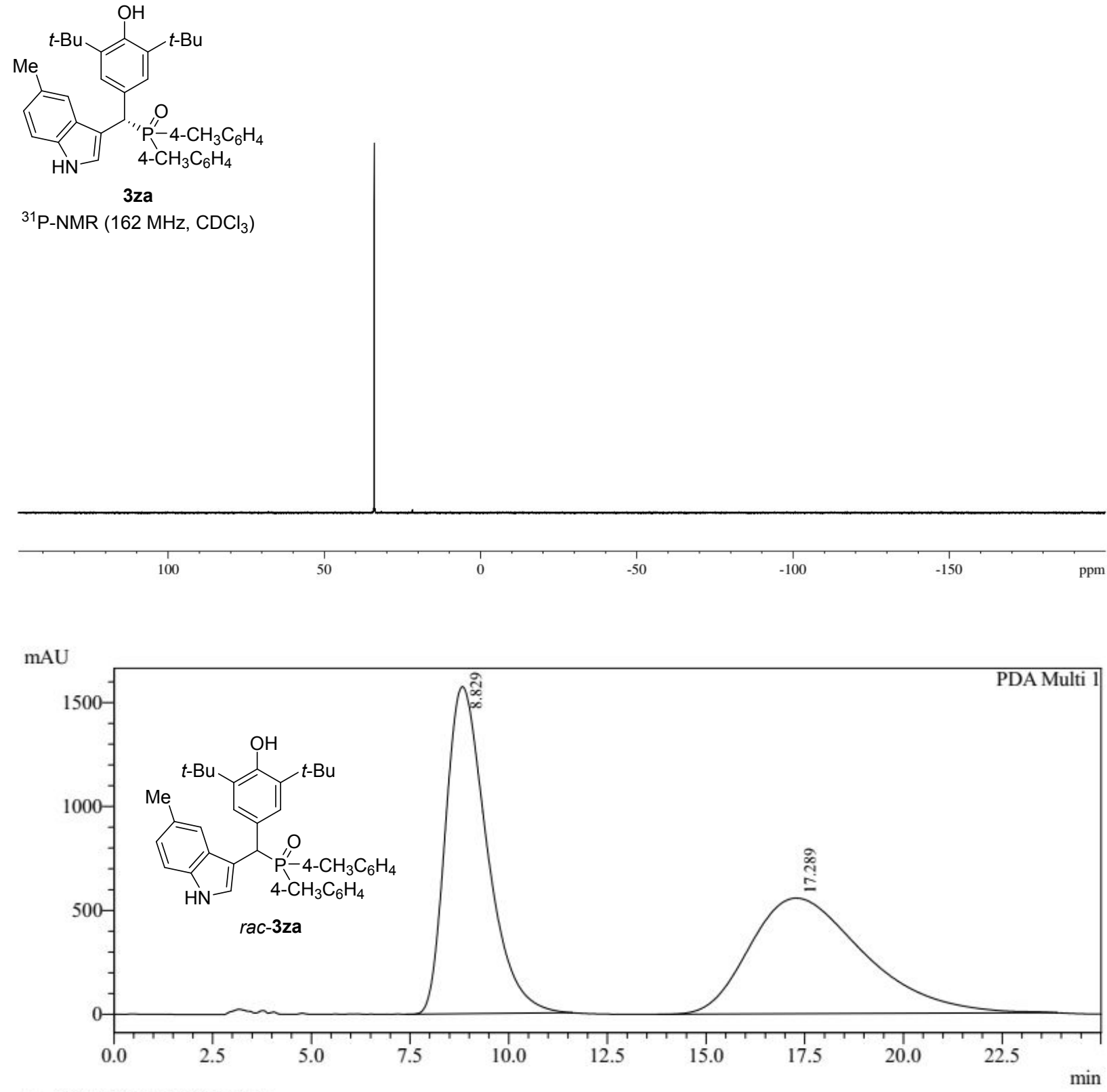

1 PDA Multi $1 / 230 \mathrm{~nm}, 4 \mathrm{~nm}$

\begin{tabular}{|c|c|c|c|c|}
\hline \multicolumn{5}{|c|}{ PDA Chl 230nm } \\
\hline Peak\# & Ret. Time & Area & Height & Area \% \\
\hline 1 & 8.829 & 114645184 & 1575901 & 50.502 \\
\hline 2 & 17.289 & 112365128 & 557144 & 49.498 \\
\hline Total & & 227010312 & 2133044 & 100.000 \\
\hline
\end{tabular}


mAU

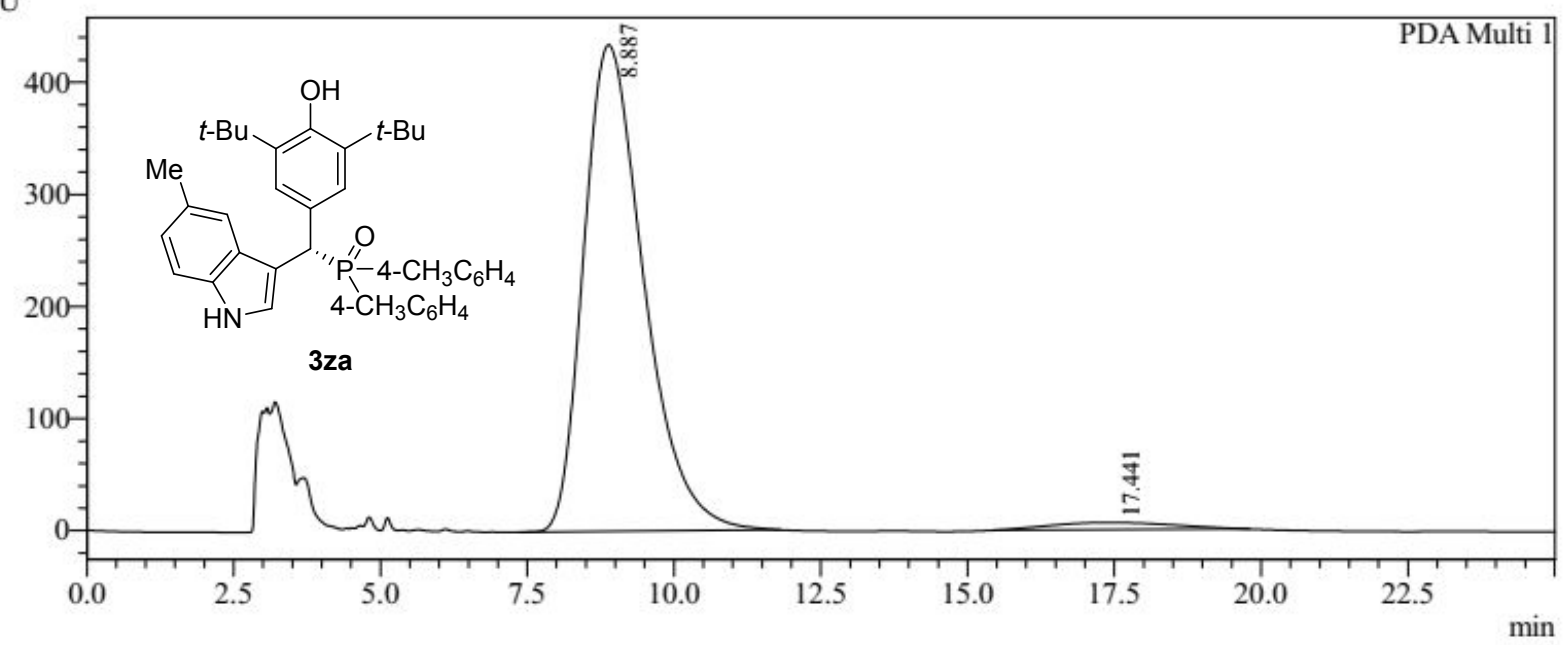

1 PDA Multi $1 / 230 \mathrm{~nm}, 4 \mathrm{~nm}$

\begin{tabular}{|c|c|c|c|c|}
\hline \multicolumn{5}{|c|}{ PDA Chl 230nm } \\
\hline Peak\# & Ret Time & Area & Height & Area \% \\
\hline 1 & 8.887 & 30981793 & 433936 & 97.036 \\
\hline 2 & 17.441 & 946411 & 6383 & 2.964 \\
\hline Total & & 31928205 & 440319 & 100.000 \\
\hline
\end{tabular}
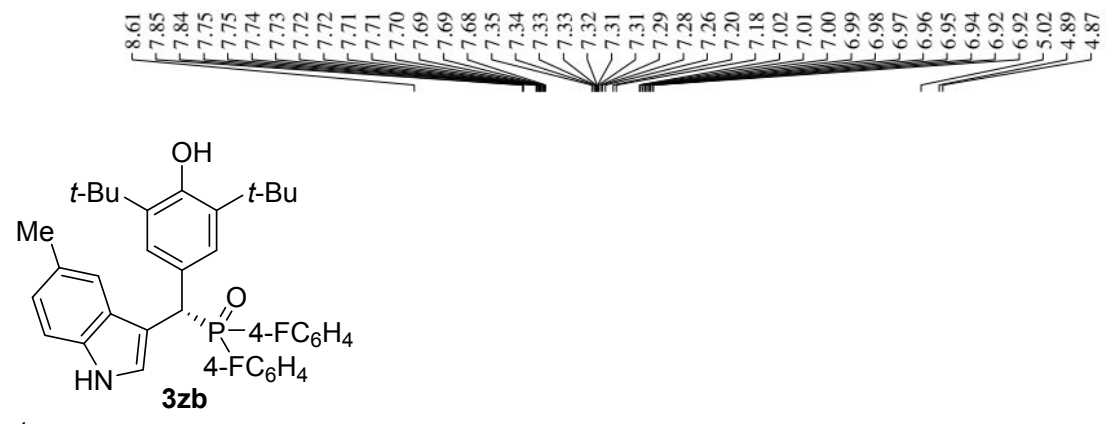

${ }^{1} \mathrm{H}-\mathrm{NMR}\left(400 \mathrm{MHz}, \mathrm{CDCl}_{3}\right)$

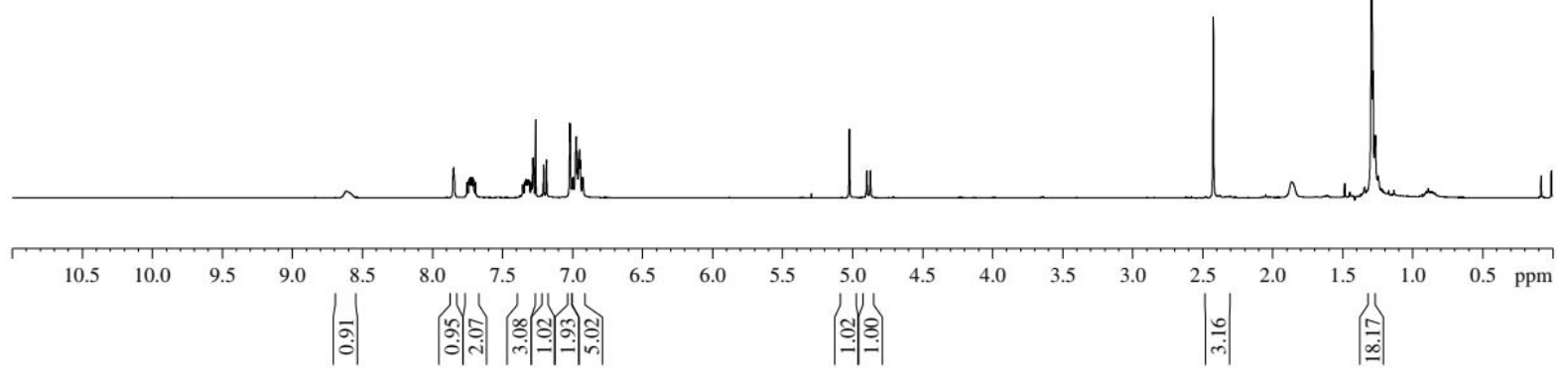



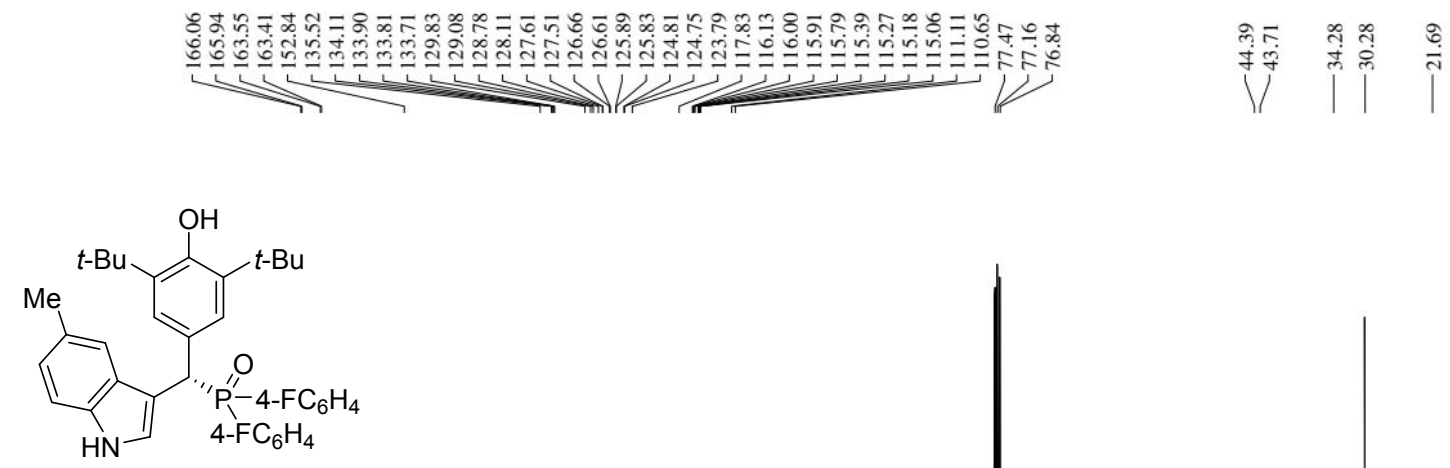

3zb

${ }^{13} \mathrm{C}\left\{{ }^{1} \mathrm{H}\right\}-\mathrm{NMR}\left(100 \mathrm{MHz}, \mathrm{CDCl}_{3}\right)$

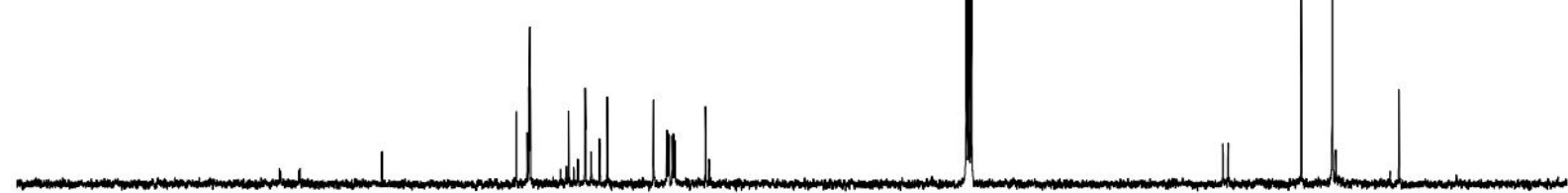

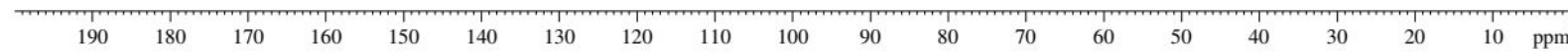

这

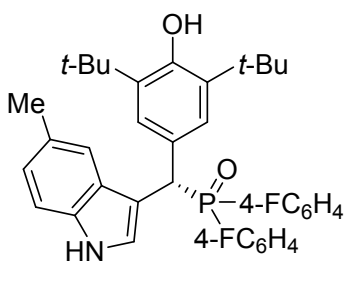

$3 z b$

${ }^{19} \mathrm{~F}-\mathrm{NMR}\left(376 \mathrm{MHz}, \mathrm{CDCl}_{3}\right)$

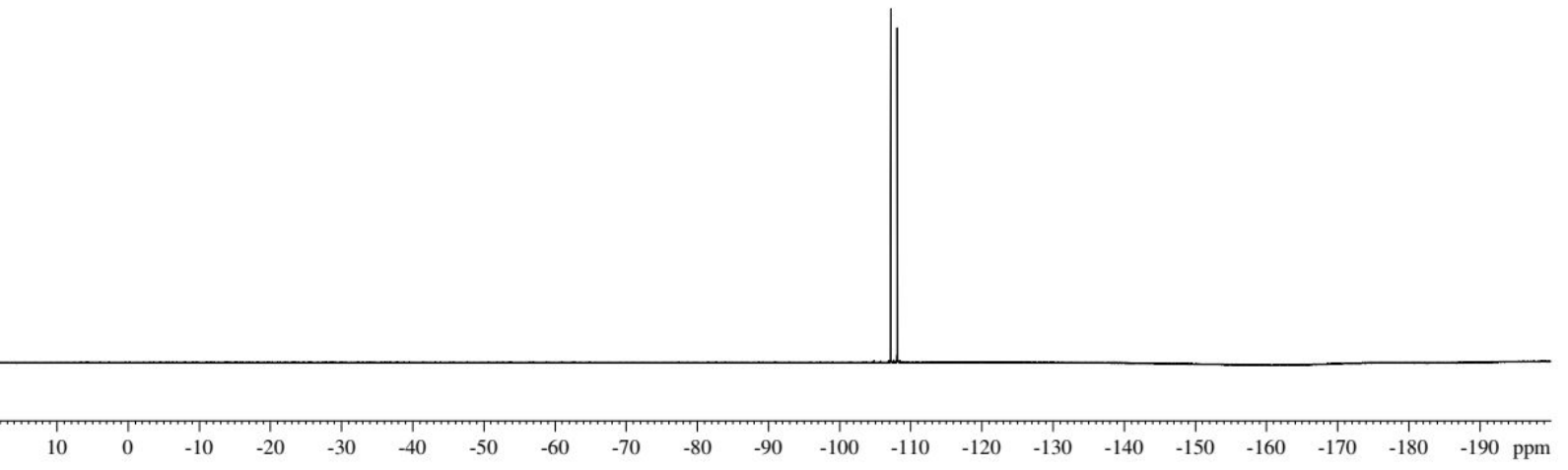



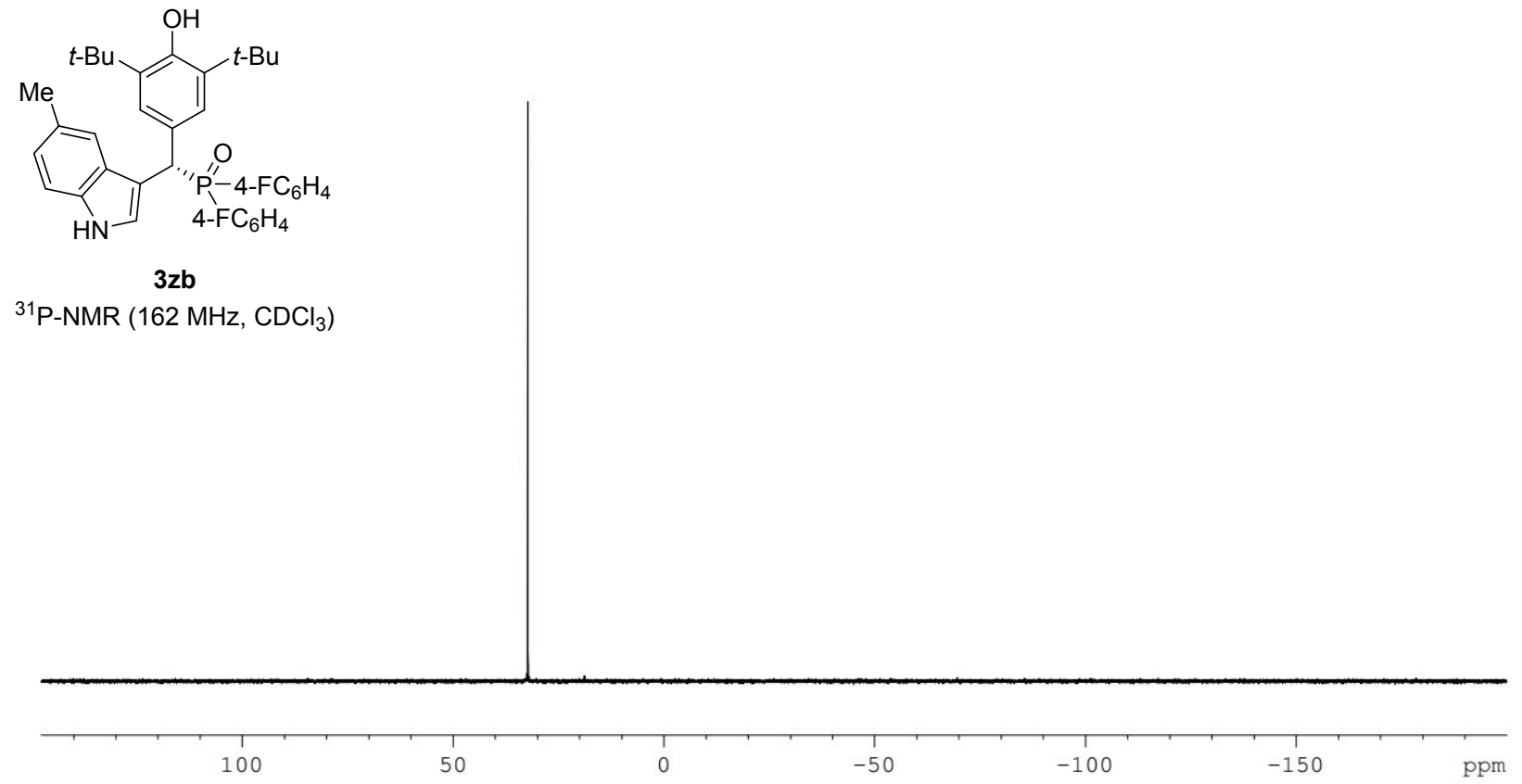

mAU

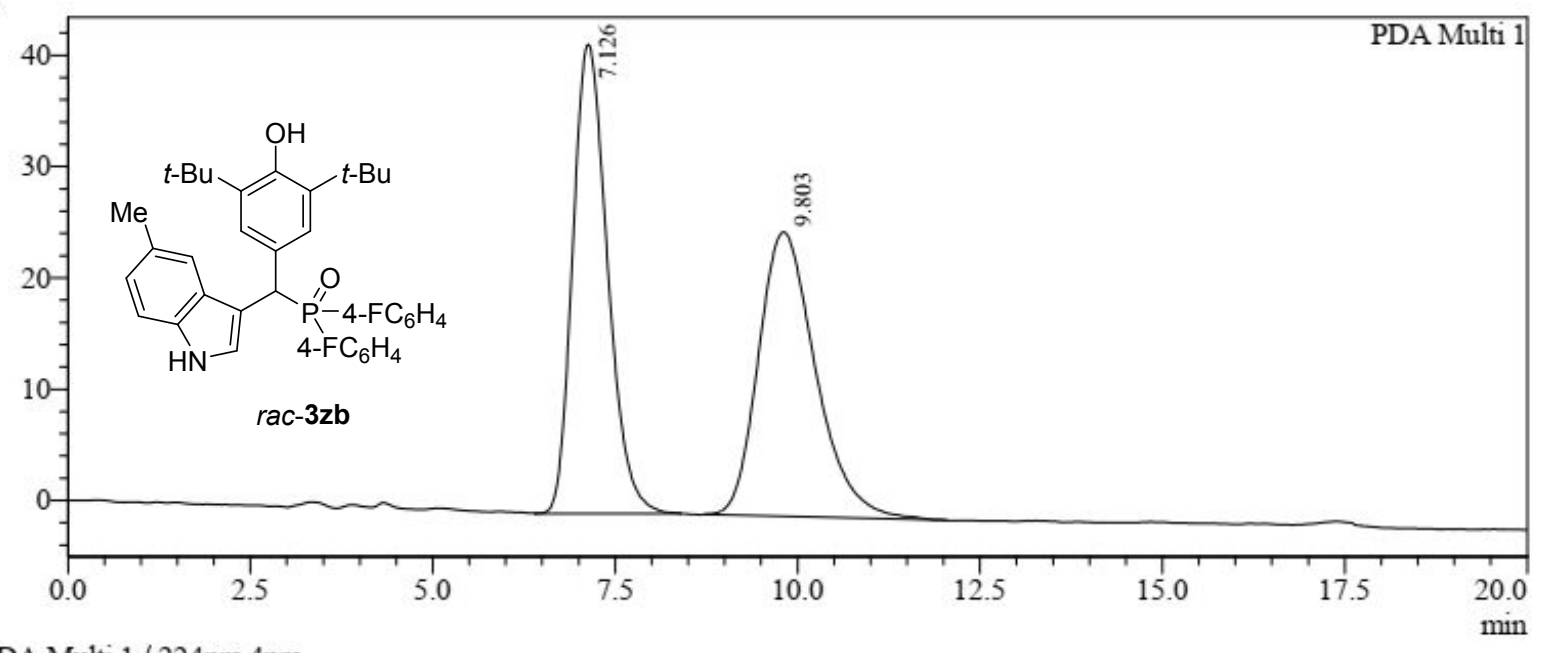

1 PDA Multi $1 / 224 \mathrm{~nm} 4 \mathrm{~nm}$

PeakTable

Ch1 $224 \mathrm{~nm} 4 \mathrm{~nm}$
\begin{tabular}{|r|r|r|r|r|}
\hline Peak\# & Ret. Time & \multicolumn{1}{|c|}{ Area } & Height & Area $\%$ \\
\hline 1 & 7.126 & 1398147 & 42132 & 50.091 \\
\hline 2 & 9.803 & 1393092 & 25520 & 49.909 \\
\hline Total & & 2791239 & 67653 & 100.000 \\
\hline
\end{tabular}


mAU

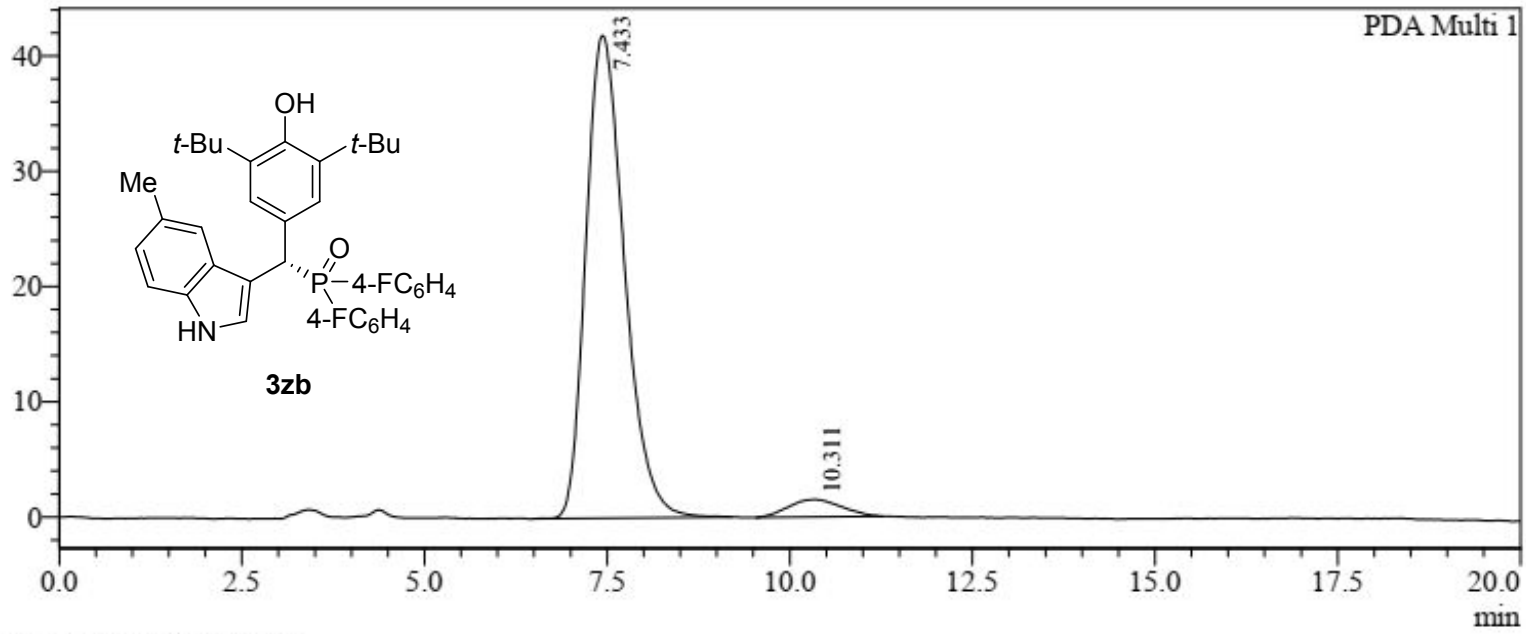

1 PDA Multi 1 / 224nm 4nm

\begin{tabular}{|c|c|c|c|c|}
\hline \multicolumn{5}{|c|}{ Ch1 224nm 4nm } \\
\hline Peak\# & Ret. Time & Area & Height & Area \% \\
\hline 1 & 7.433 & 1539210 & 41872 & 95.287 \\
\hline 2 & 10.311 & 76127 & 1512 & 4.713 \\
\hline Total & & 1615338 & 43384 & 100.000 \\
\hline
\end{tabular}

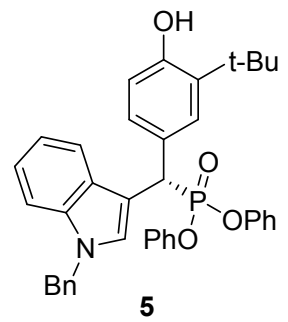

${ }^{1} \mathrm{H}-\mathrm{NMR}\left(400 \mathrm{MHz}, \mathrm{CDCl}_{3}\right)$

lathylu
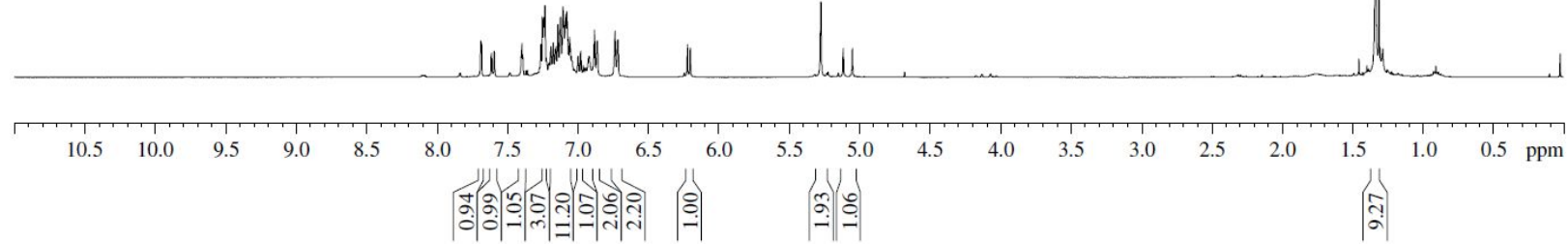

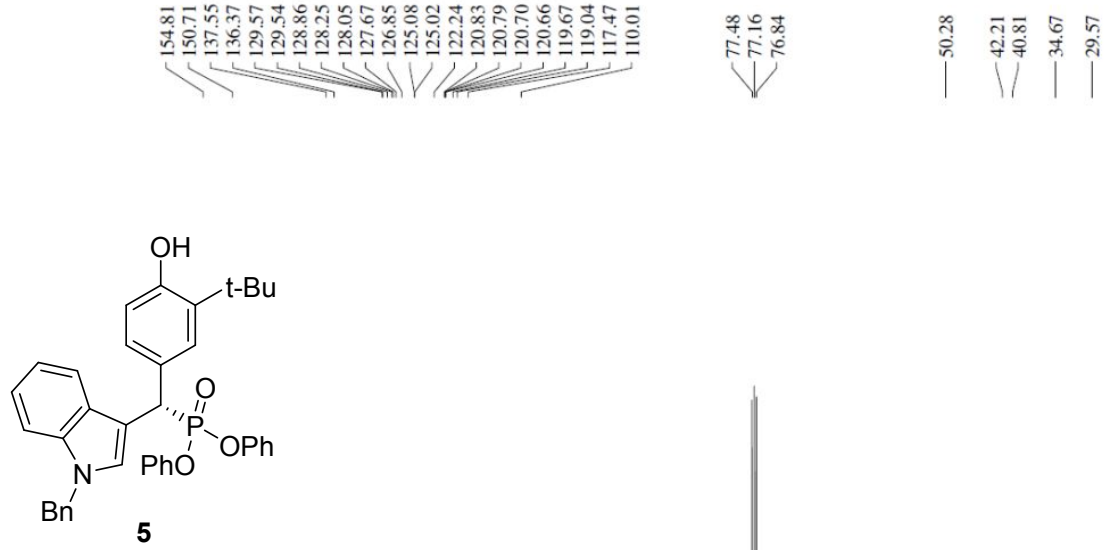

${ }^{13} \mathrm{C}\left\{{ }^{1} \mathrm{H}\right\}-\mathrm{NMR}\left(100 \mathrm{MHz}, \mathrm{CDCl}_{3}\right)$
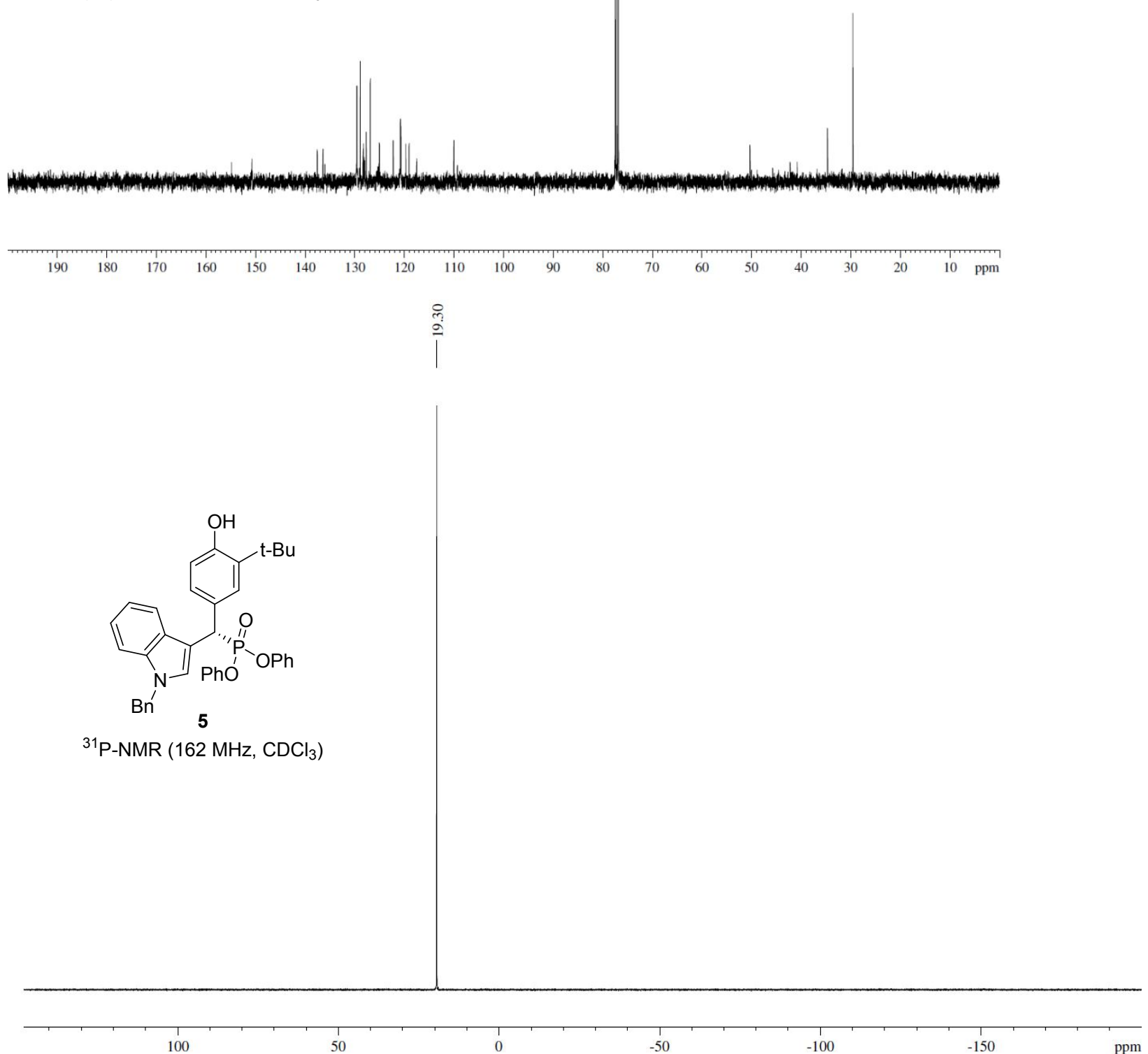
mAU

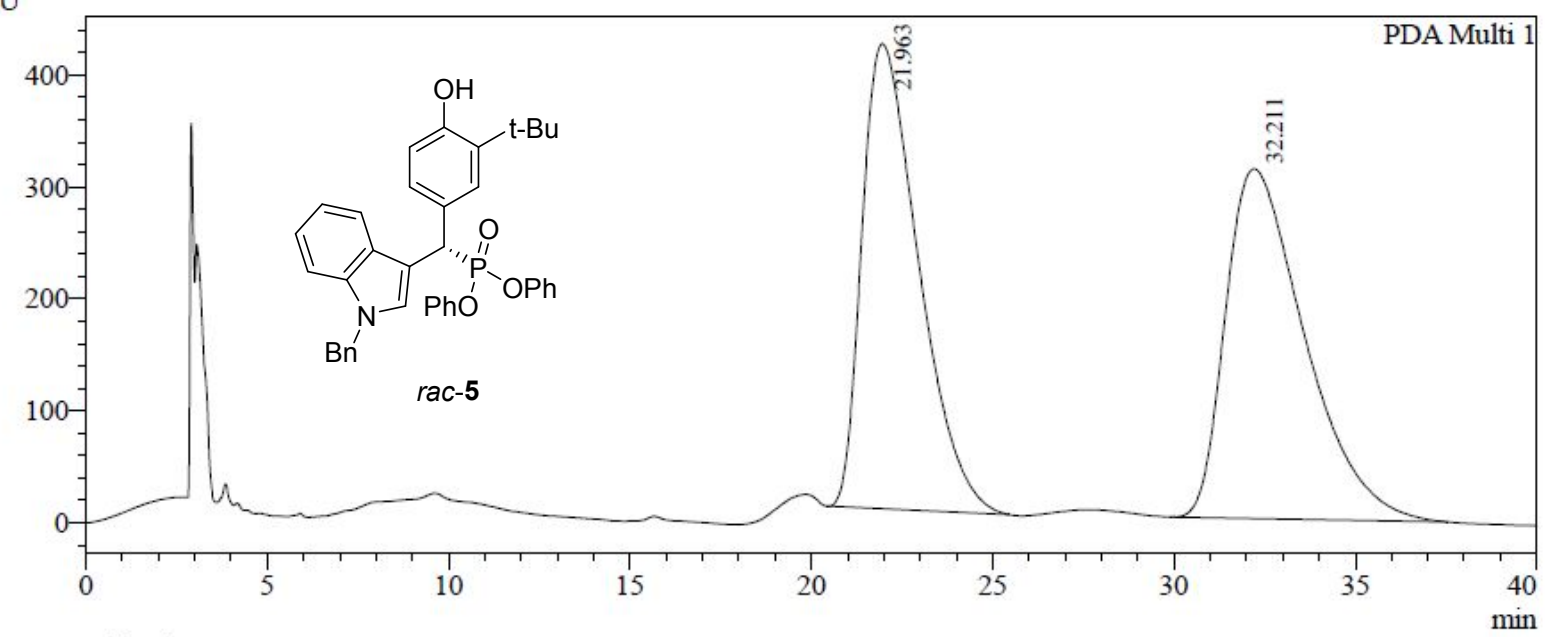

1 PDA Multi $1 / 230 \mathrm{~nm}, 4 \mathrm{~nm}$

PeakTable

PDA Ch1 230 nm
\begin{tabular}{|r|r|r|r|r|}
\hline Peak\# & Ret. Time & Area & Height & \multicolumn{1}{|c|}{ Area $\%$} \\
\hline 1 & 21.963 & 45143783 & 415336 & 48.703 \\
\hline 2 & 32.211 & 47547663 & 312472 & 51.297 \\
\hline Total & & 92691446 & 727808 & 100.000 \\
\hline
\end{tabular}

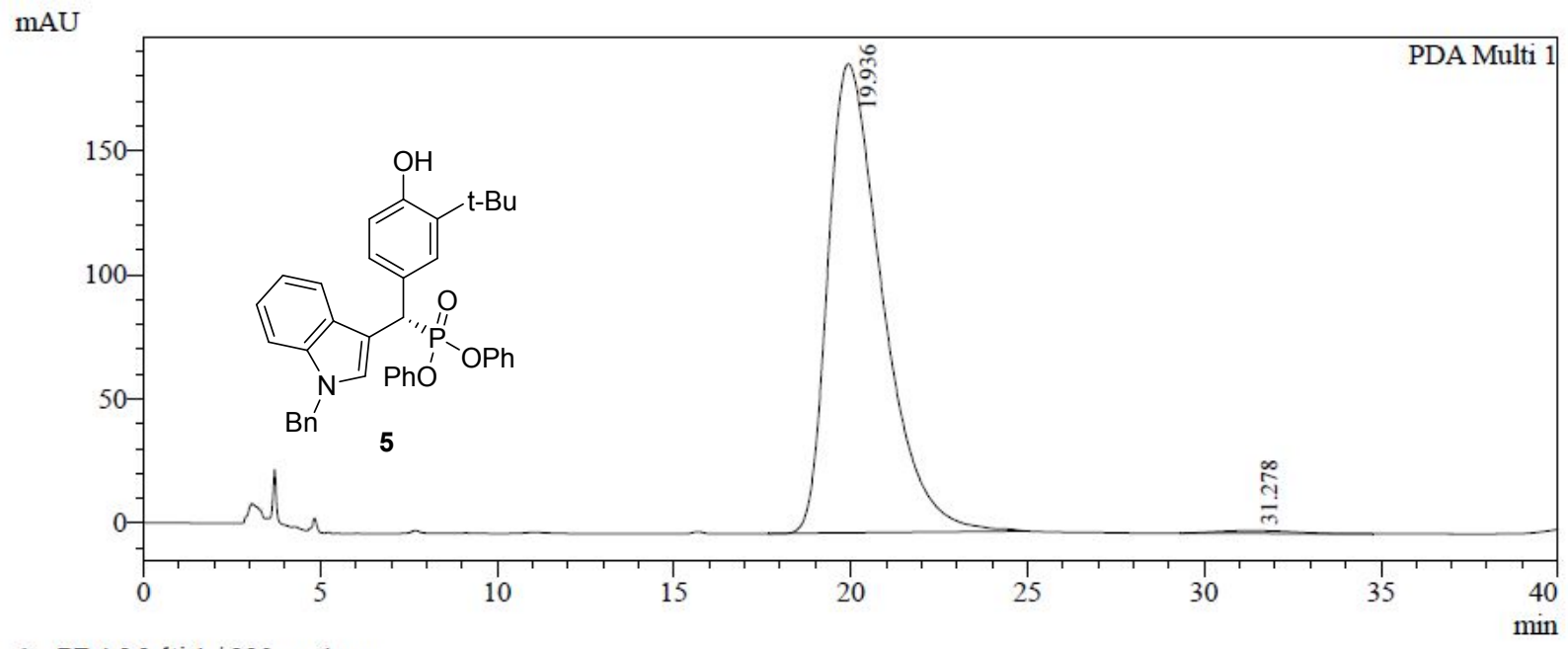

1 PDA Multi $1 / 230 \mathrm{~nm}, 4 \mathrm{~nm}$

PeakTable

PDA Ch1 $230 \mathrm{~nm}$
\begin{tabular}{|r|r|r|r|r|}
\hline Peak\# & Ret. Time & \multicolumn{1}{|c|}{ Area } & \multicolumn{1}{c|}{ Height } & Area \% \\
\hline 1 & 19.936 & 20206879 & 188967 & 99.219 \\
\hline 2 & 31.278 & 158992 & 1034 & 0.781 \\
\hline Total & & 20365870 & 190001 & 100.000 \\
\hline
\end{tabular}


<smiles>CC(C)(C)c1cc(C(c2ccc3ccccc3c2O)P(=O)(c2ccccc2)c2ccccc2)cc(C(C)(C)C)c1O</smiles>

${ }^{1} \mathrm{H}-\mathrm{NMR}\left(400 \mathrm{MHz}, \mathrm{CDCl}_{3}\right)$
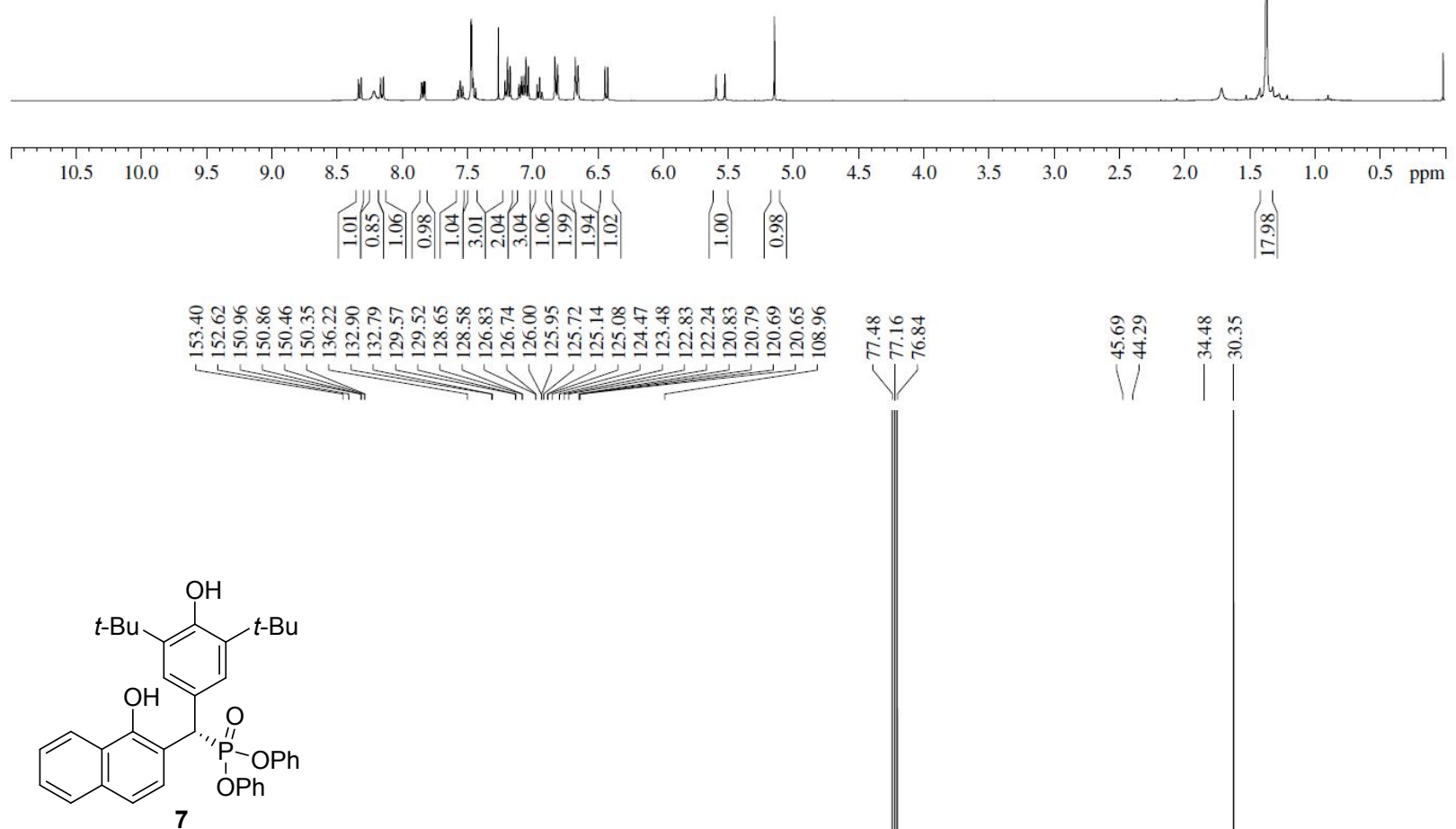

${ }^{13} \mathrm{C}\left\{{ }^{1} \mathrm{H}\right\}-\mathrm{NMR}\left(100 \mathrm{MHz}, \mathrm{CDCl}_{3}\right)$

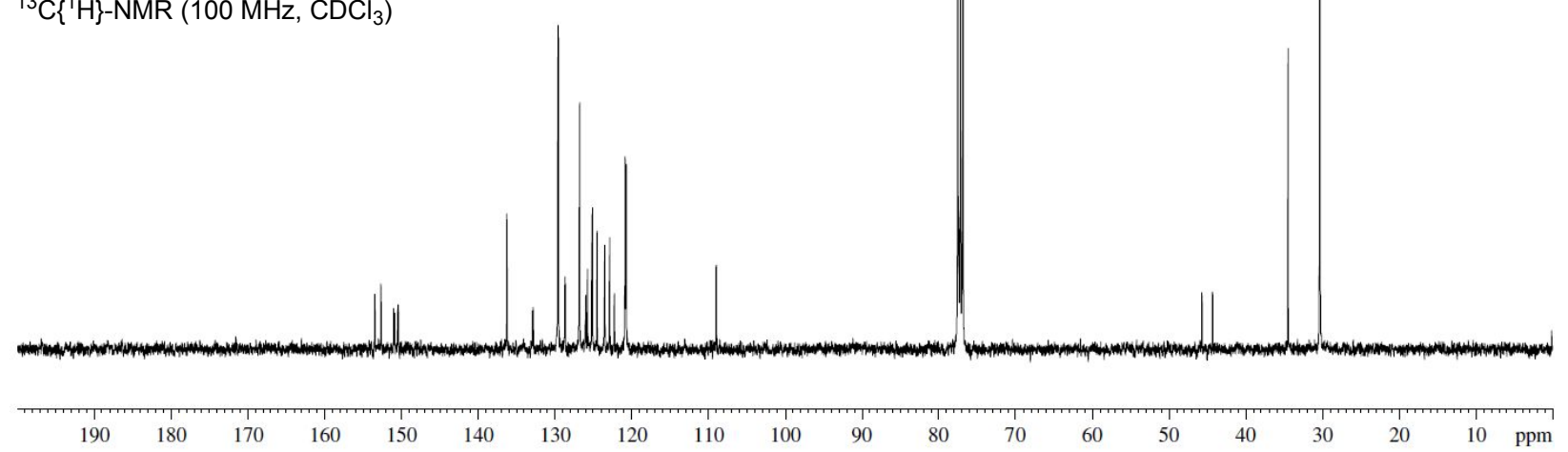




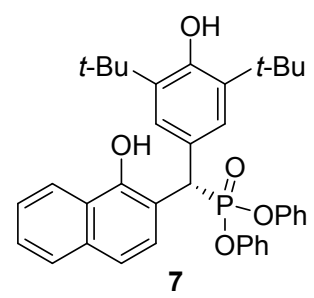

${ }^{31} \mathrm{P}-\mathrm{NMR}\left(162 \mathrm{MHz}, \mathrm{CDCl}_{3}\right.$ )
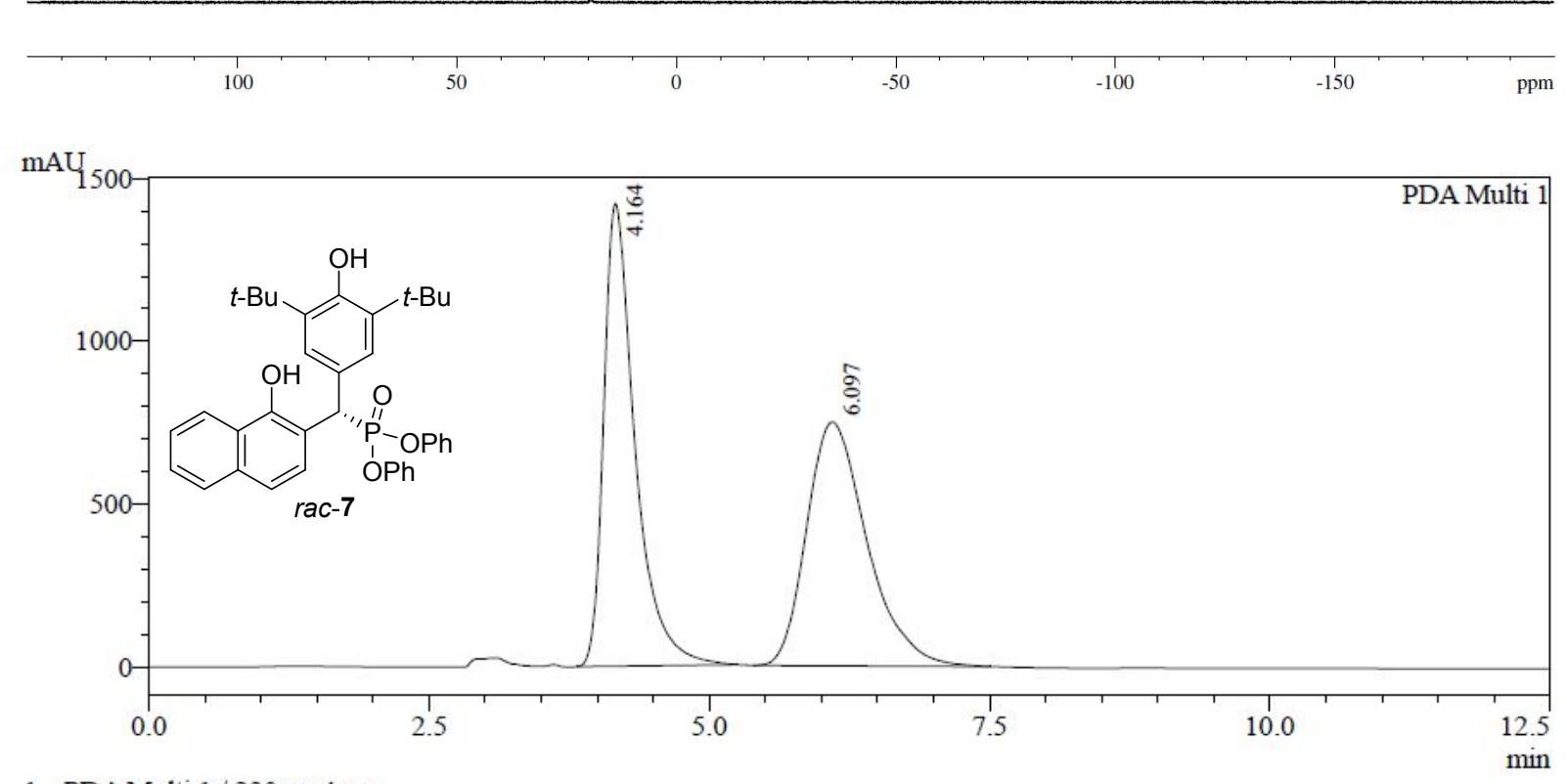

1 PDA Multi $1 / 230 \mathrm{~nm}, 4 \mathrm{~nm}$

PDA Ch1 230nm
\begin{tabular}{|r|r|r|r|r|}
\hline \multicolumn{1}{|c|}{ Peak\# } & ReakTable \\
\hline 1 & 4.164 & 27623463 & 1420152 & \multicolumn{1}{|c|}{ Area $\%$} \\
\hline 2 & 6.097 & 28001559 & 748353 & 50.660 \\
\hline Total & & 55625022 & 2168505 & 100.000 \\
\hline
\end{tabular}


mAU

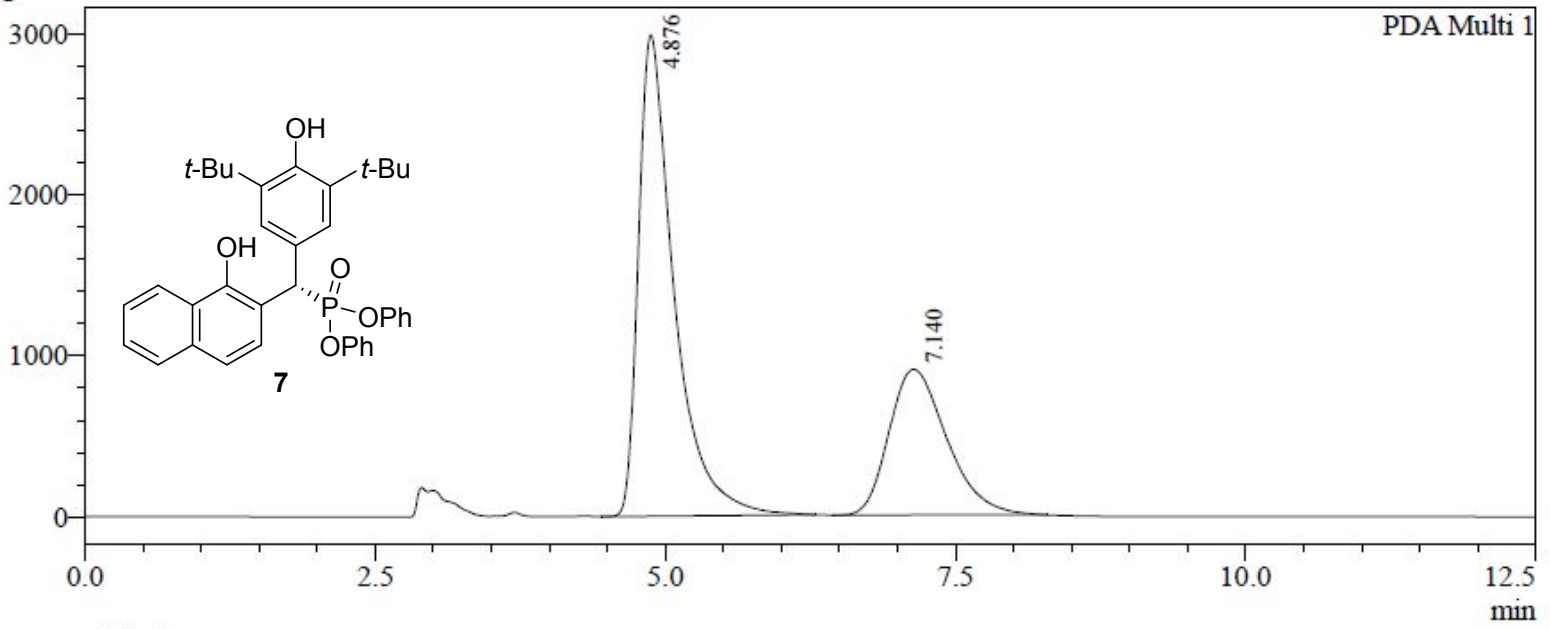

1 PDA Multi $1 / 230 \mathrm{~nm}, 4 \mathrm{~nm}$

\begin{tabular}{|c|c|c|c|c|}
\hline \multicolumn{5}{|c|}{ PDA Ch1 230nm } \\
\hline Peak\# & Ret. Time & Area & Height & Area $\%$ \\
\hline 1 & 4.876 & 63949094 & 2987545 & 67.113 \\
\hline 2 & 7.140 & 31337102 & 904175 & 32.887 \\
\hline Total & & 95286197 & 3891719 & 100.000 \\
\hline
\end{tabular}

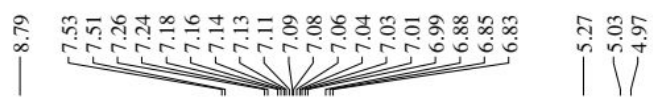<smiles>[C-]c1cc(C(c2c[nH]c3ccccc23)P(=O)(O)c2ccccc2)cc(C)c1O</smiles>

$11 \mathrm{a}$

${ }^{1} \mathrm{H}-\mathrm{NMR}\left(400 \mathrm{MHz}, \mathrm{CDCl}_{3}\right)$

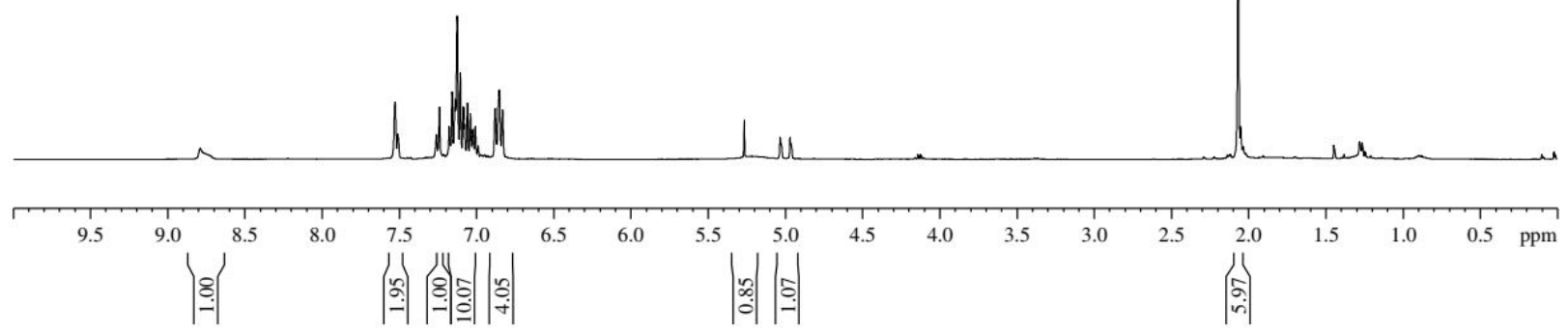




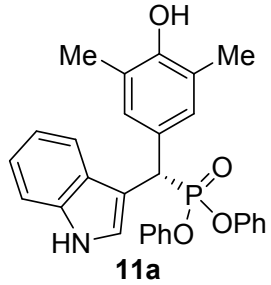

${ }^{13} \mathrm{C}\left\{{ }^{1} \mathrm{H}\right\}-\mathrm{NMR}\left(100 \mathrm{MHz}, \mathrm{CDCl}_{3}\right)$
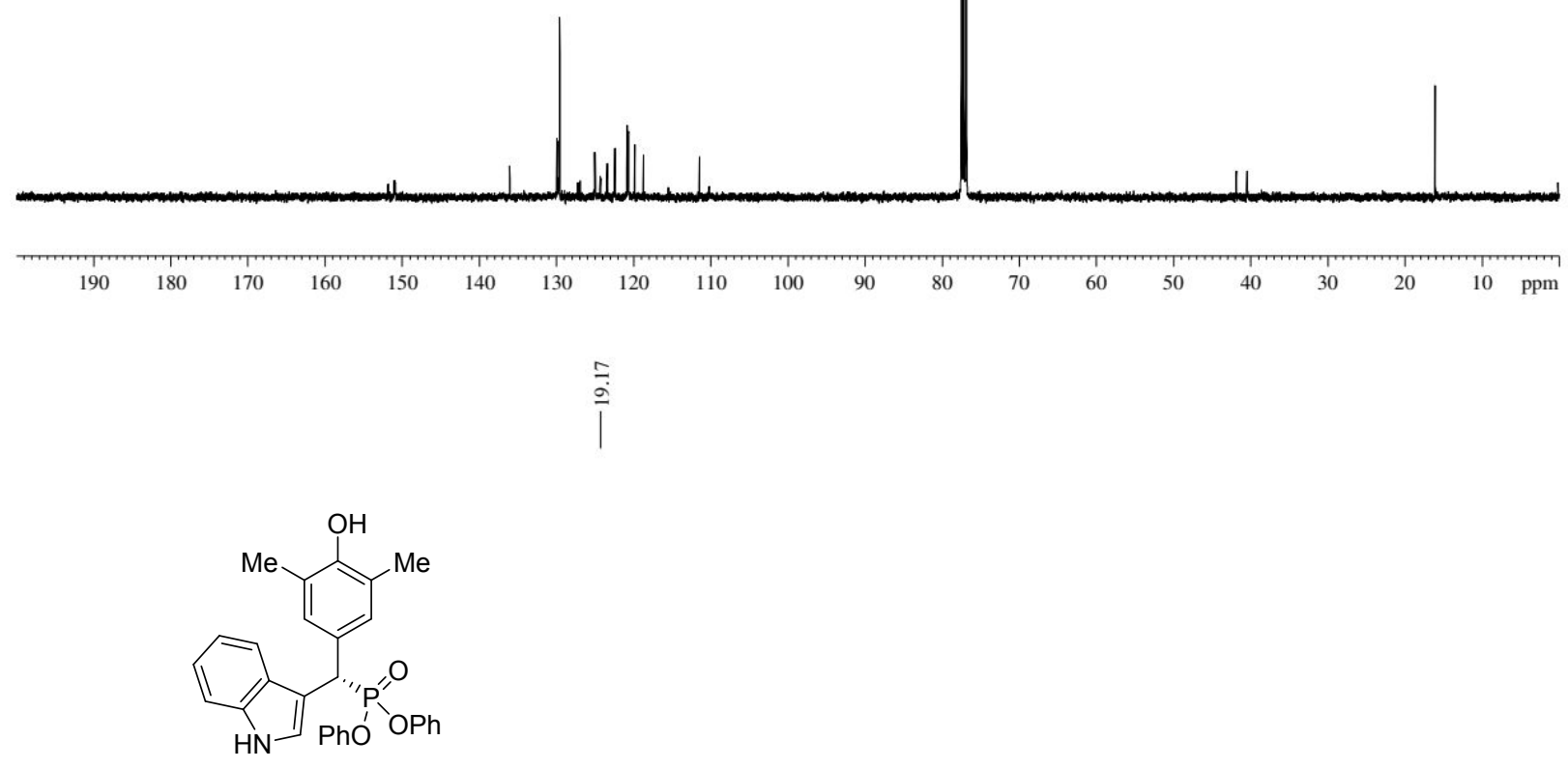

$11 \mathrm{a}$

${ }^{31} \mathrm{P}-\mathrm{NMR}\left(162 \mathrm{MHz}, \mathrm{CDCl}_{3}\right.$ )

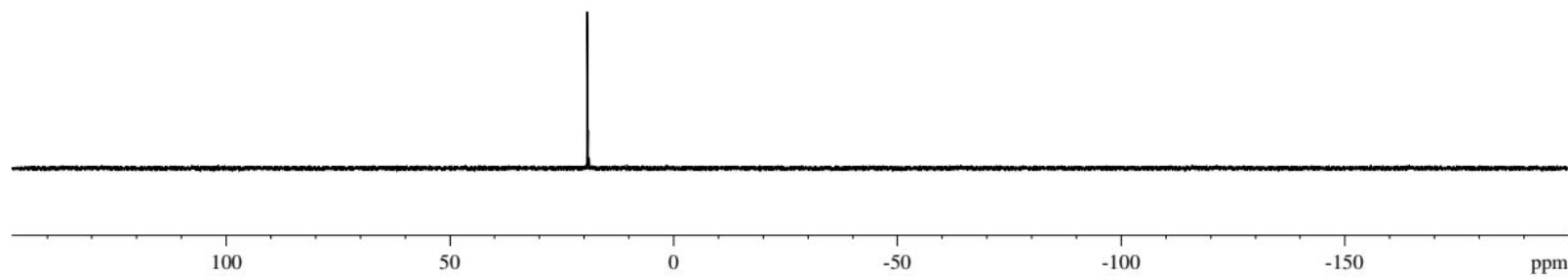


mAU

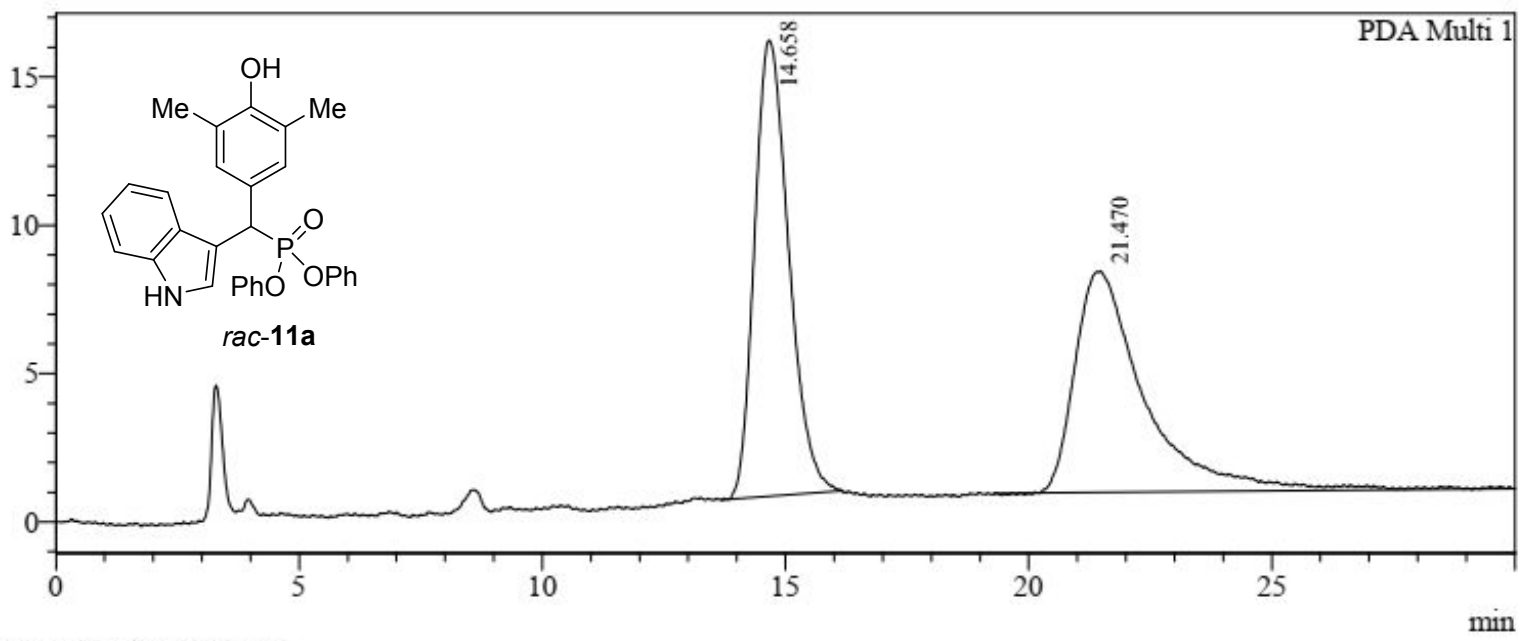

1 PDA Multi $1 / 205 \mathrm{~nm} 4 \mathrm{~nm}$

Ch1 205nm 4nm
\begin{tabular}{|r|r|r|r|r|}
\hline \multicolumn{1}{|c|}{ PeakTable } \\
\hline 1 & Ret. Time & Area & \multicolumn{1}{c|}{ Height } & \multicolumn{1}{c|}{ Area $\%$} \\
\hline 2 & 14.658 & 752771 & 15363 & 50.190 \\
\hline Total & 21.470 & 747079 & 7459 & 49.810 \\
\hline & & 1499851 & 22822 & 100.000 \\
\hline
\end{tabular}

mAU

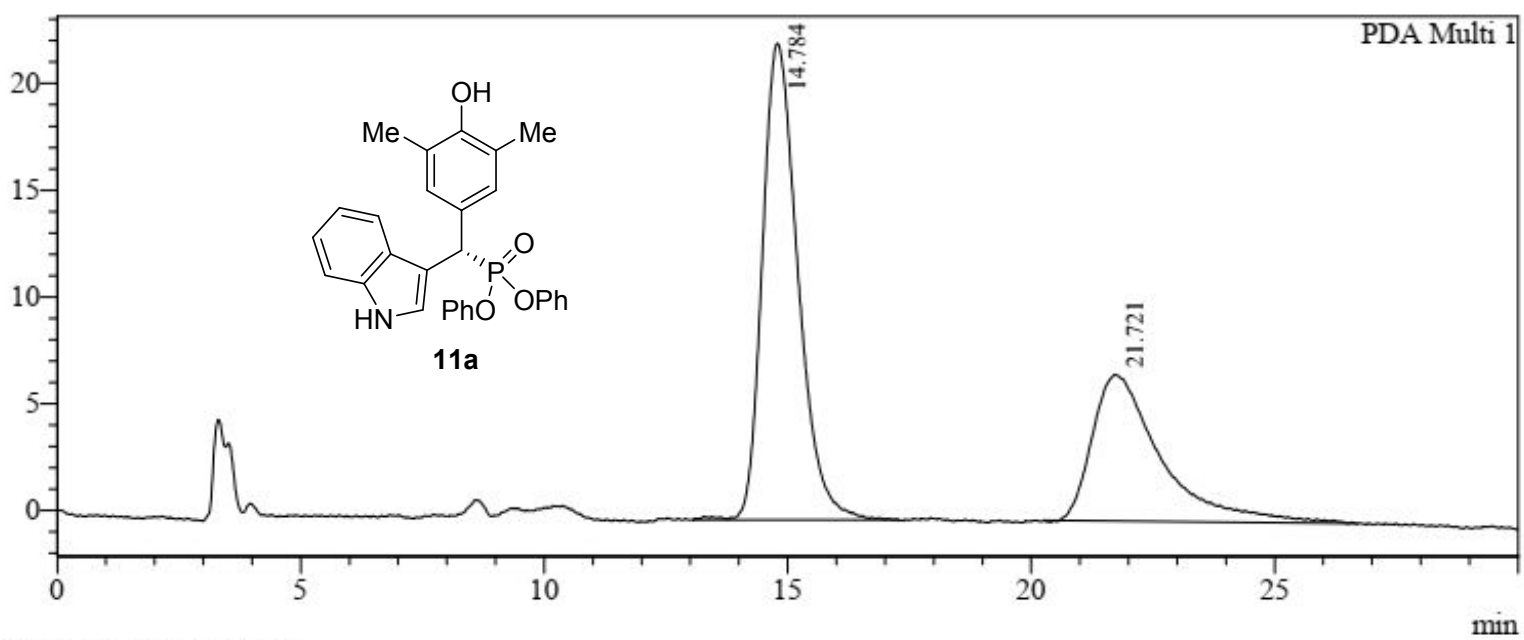

1 PDA Multi $1 / 205 \mathrm{~nm} 4 \mathrm{~nm}$

PeakTable

Ch1 $205 \mathrm{~nm} 4 \mathrm{~nm}$
\begin{tabular}{|r|r|r|r|r|}
\hline Peak\# & Ret. Time & \multicolumn{1}{|c|}{ Area } & Height & Area $\%$ \\
\hline 1 & 14.784 & 1135018 & 22302 & 62.876 \\
\hline 2 & 21.721 & 670165 & 6865 & 37.124 \\
\hline Total & & 1805184 & 29167 & 100.000 \\
\hline
\end{tabular}




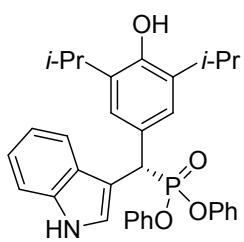

11b

${ }^{1} \mathrm{H}-\mathrm{NMR}\left(400 \mathrm{MHz}, \mathrm{CDCl}_{3}\right)$
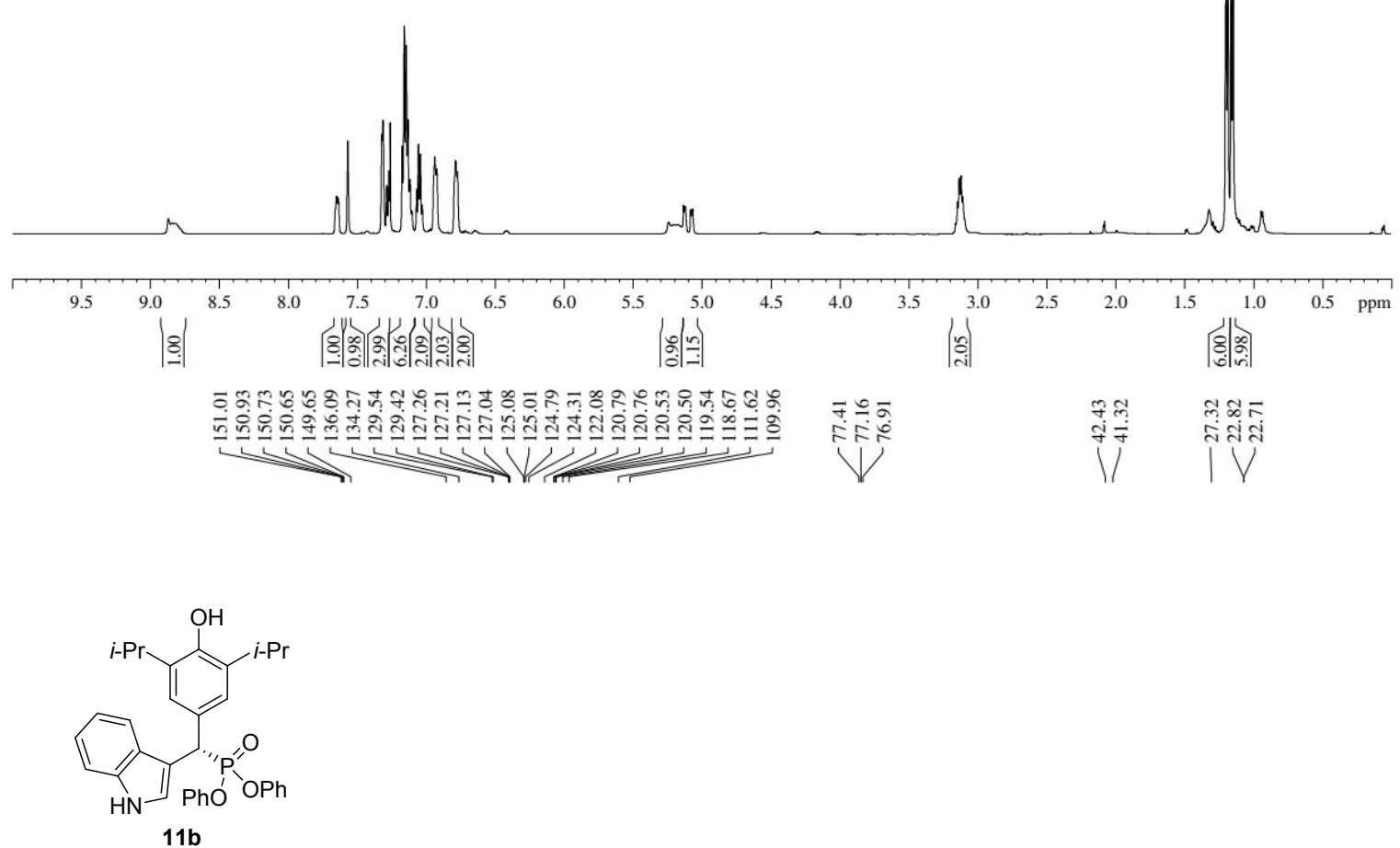

${ }^{13} \mathrm{C}\left\{{ }^{1} \mathrm{H}\right\}-\mathrm{NMR}(100 \mathrm{MHz}, \mathrm{CDCl})$
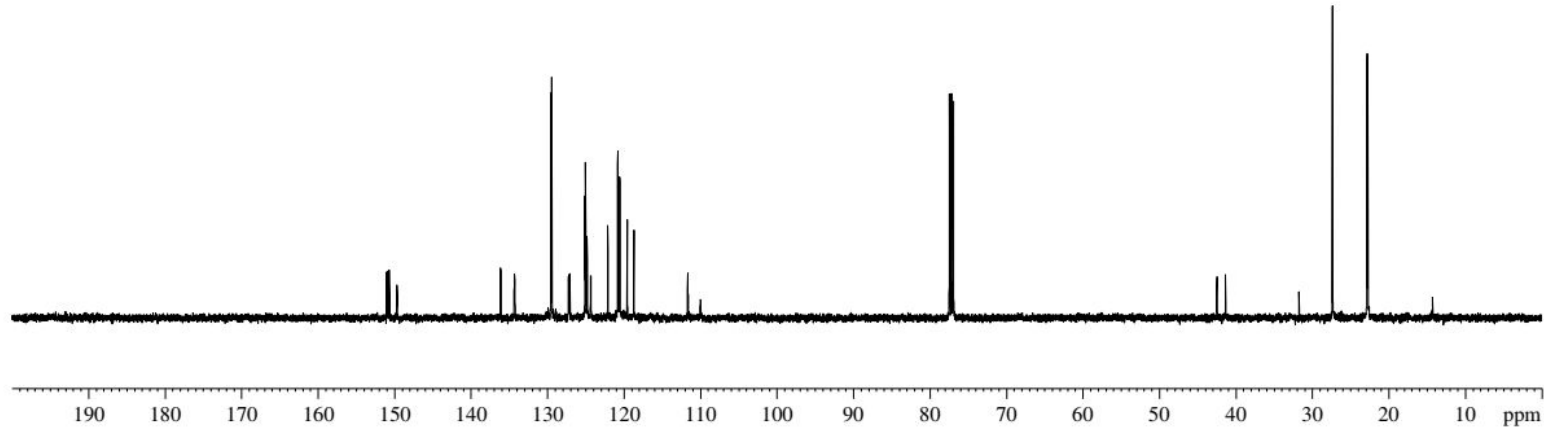


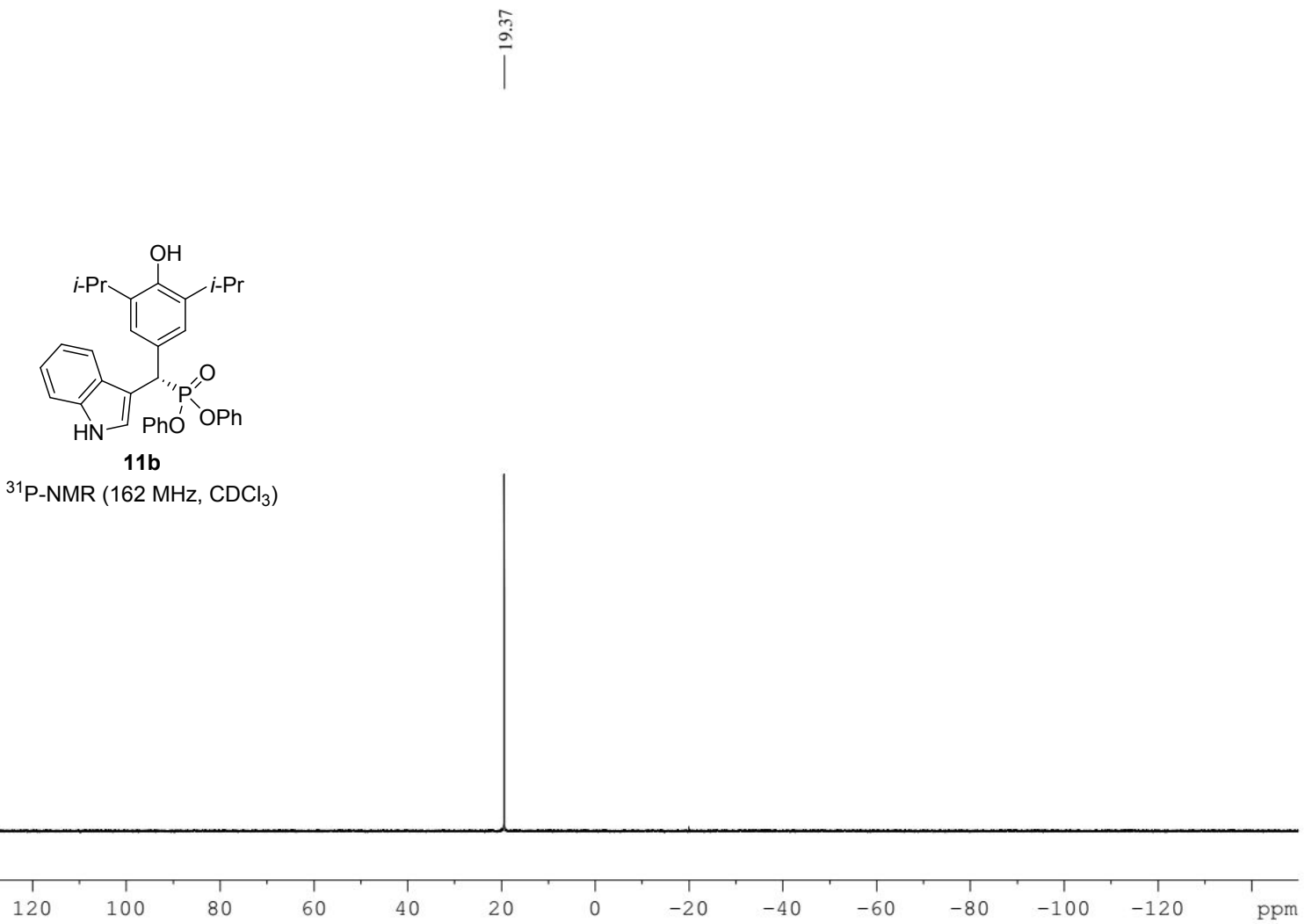

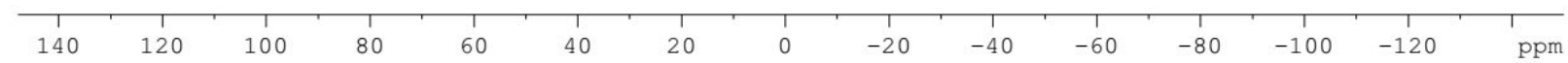

$\mathrm{mAU}$

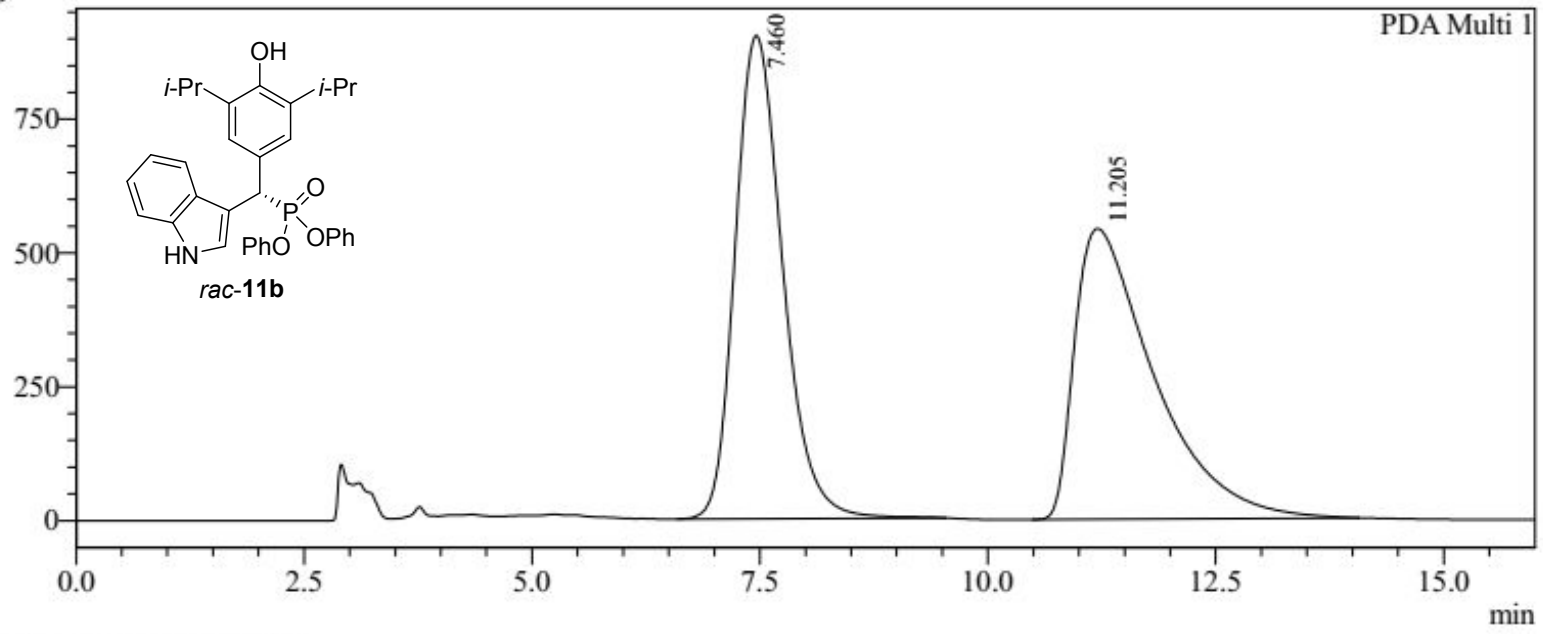

1 PDA Multi $1 / 230 \mathrm{~nm}, 4 \mathrm{~nm}$

\begin{tabular}{|c|c|c|c|c|}
\hline \multicolumn{5}{|c|}{ PDA Chl 230nm } \\
\hline Peak\# & Ret. Time & Area & Height & Area $\%$ \\
\hline 1 & 7.460 & 32788845 & 902359 & 49.951 \\
\hline 2 & 11.205 & 32852967 & 542775 & 50.049 \\
\hline Total & & 65641812 & 1445134 & 100.000 \\
\hline
\end{tabular}




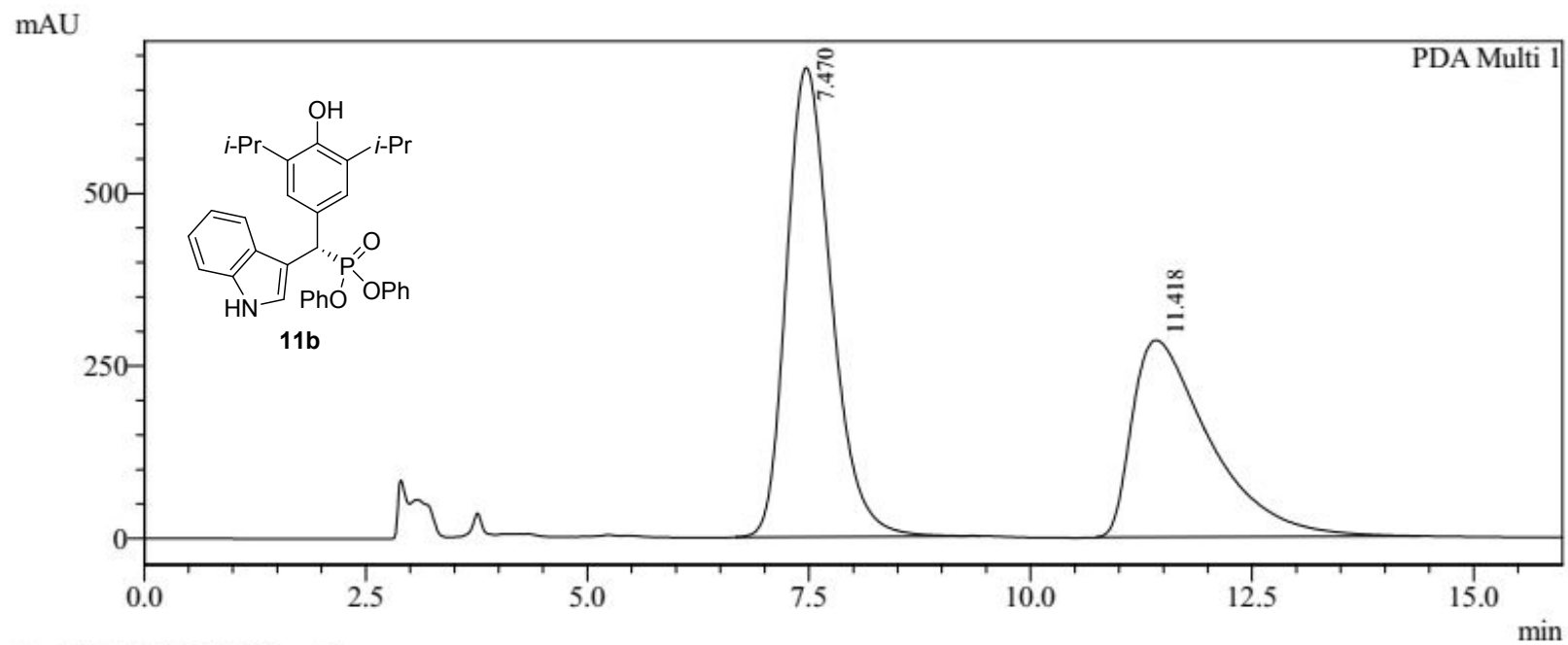

1 PDA Multi $1 / 230 \mathrm{~nm}, 4 \mathrm{~nm}$

\begin{tabular}{|c|c|c|c|c|}
\hline \multicolumn{5}{|c|}{ PDAChl 230nm } \\
\hline Peak\# & Ret. Time & Area & Height & Area \% \\
\hline 1 & 7.470 & 23963739 & 679851 & 57.945 \\
\hline 2 & 11.418 & 17392370 & 285192 & 42.055 \\
\hline Total & & 41356109 & 965043 & 100.000 \\
\hline
\end{tabular}

\section{Single Crystal X-ray Diffraction Analysis of 3z:}

A single crystal of $\mathbf{3 z}$ was mounted and the diffraction data were collected at $273 \mathrm{~K}$ on a Bruker APEX-II CCD diffractometer using Bruker SAINT software. The crystallographic refinement parameters are given below:

\section{Crystal data and structure refinement for $3 z$}

Identification code

Empirical formula

Formula weight

Temperature

Wavelength

Crystal system

Space group

Unit cell dimensions

Volume

$\mathrm{Z}$

Density (calculated) 3z

$\mathrm{C}_{72} \mathrm{H}_{82} \mathrm{~N}_{2} \mathrm{O}_{5} \mathrm{P}_{2}$

1117.34

273(2) K

$0.71073 \mathrm{~A}^{\mathrm{o}}$

Orthorhombic

P $2{ }_{1} 2{ }_{1} 2_{1}$

$a=10.8366(7) \mathrm{A}^{\mathrm{o}} \quad \alpha=90^{\circ}$.

$b=12.9616(9) \mathrm{A}^{\circ} \quad \beta=90^{\circ}$.

$c=45.140(3) \mathrm{A}^{\mathrm{o}} \quad \gamma=90^{\circ}$.

$6340.3(7) \mathrm{A}^{\mathrm{o}^{3}}$

4

$1.171 \mathrm{~g} / \mathrm{cm}^{3}$ 


$\begin{array}{ll}\text { Absorption coefficient } & 0.120 \mathrm{~mm}^{-1} \\ \mathrm{~F}(000) & 2392.0 \\ \text { Crystal size } & 0.3 \times 0.2 \times 0.1 \mathrm{~mm}^{3} \\ \text { Theta range for data collection } & 2.39 \text { to } 26.95^{\circ} . \\ \text { Index ranges } & -14 \leq \mathrm{h} \leq 14,-17 \leq \mathrm{k} \leq 17,-60 \leq 1 \leq 60 \\ \text { Reflections collected } & 138261 \\ \text { Independent reflections } & 15839 \text { Rint }=0.0890, \text { Rsigma }=0.0515] \\ \text { Completeness to theta }=26.95^{\circ} & 100.0 \% \\ \text { Refinement method } & \text { Full-matrix least-squares on } \mathrm{F}^{2} \\ \text { Data / restraints / parameters } & 15839 / 0 / 702 \\ \text { Goodness-of-fit on F } & 1.036 \\ \text { Final R indices [I }>2 \sigma(\mathrm{I})] & \mathrm{R} 1=0.0472, \mathrm{wR} 2=0.1074 \\ \text { R indices (all data) } & \mathrm{R} 1=0.0663, \mathrm{wR} 2=0.1199 \\ \text { Absolute structure (Flack) parameter } & 0.07(3) \\ \text { Largest diff. peak and hole } & 0.59 \text { and }-0.36 \mathrm{e} . \mathrm{A}^{\mathrm{o}}{ }^{-3}\end{array}$

Atomic coordinates $\left(\times 10^{4}\right)$ and equivalent isotropic displacement parameters $\left(\mathrm{A}^{\left.\mathrm{o}^{2} \times 10^{3}\right)}\right.$ for 3z. $U(e q)$ is defined as one third of the trace of the orthogonalized $U^{\mathrm{ij}}$ tensor.

\begin{tabular}{lrrrr}
\hline P2 & $1441.5(7)$ & $4675.2(6)$ & $6072.5(2)$ & $20.12(16)$ \\
P1 & $7765.3(7)$ & $9572.4(6)$ & $7033.0(2)$ & $22.09(16)$ \\
O2 & $3258(2)$ & $6930.0(17)$ & $6509.9(5)$ & $26.6(5)$ \\
O4 & $2055(2)$ & $4695.0(19)$ & $4654.1(4)$ & $28.4(5)$ \\
O3 & $2679.6(19)$ & $5163.5(17)$ & $6096.6(5)$ & $25.9(5)$ \\
O1 & $7123(2)$ & $9663.9(18)$ & $7323.6(5)$ & $29.2(5)$ \\
N1 & $5662(3)$ & $12615(2)$ & $6922.5(6)$ & $27.9(6)$ \\
N2 & $3781(2)$ & $1832(2)$ & $6195.4(6)$ & $29.1(6)$ \\
C27 & $4998(3)$ & $7939(2)$ & $6325.4(6)$ & $19.1(6)$ \\
C20 & $6070(3)$ & $12828(2)$ & $6640.9(7)$ & $21.9(6)$ \\
C29 & $5071(3)$ & $7360(2)$ & $6029.1(6)$ & $20.8(6)$ \\
C56 & $2889(3)$ & $1093(3)$ & $6146.2(6)$ & $25.7(6)$ \\
C26 & $4111(3)$ & $7699(2)$ & $6544.1(6)$ & $20.4(6)$ \\
C50 & $2202(3)$ & $2668(2)$ & $5983.2(6)$ & $20.3(6)$ \\
C19 & $6033(3)$ & $13724(2)$ & $6474.2(7)$ & $26.5(7)$ \\
C52 & $813(3)$ & $1025(2)$ & $5949.5(6)$ & $23.4(6)$ \\
C15 & $6591(2)$ & $11903(2)$ & $6528.3(6)$ & $19.4(6)$ \\
C16 & $7078(3)$ & $11898(2)$ & $6238.5(6)$ & $19.4(6)$
\end{tabular}




\begin{tabular}{|c|c|c|c|c|}
\hline $\mathrm{C} 43$ & 808.1(19) & $4348.7(16)$ & $6431.3(3)$ & $22.6(6)$ \\
\hline $\mathrm{C} 48$ & $-161.7(18)$ & $3663.5(15)$ & $6474.9(3)$ & $24.4(6)$ \\
\hline $\mathrm{C} 47$ & $-575.7(18)$ & $3454.2(16)$ & $6760.1(4)$ & $26.8(7)$ \\
\hline C46 & $-20(2)$ & $3930(2)$ & $7001.7(3)$ & 41.4(9) \\
\hline $\mathrm{C} 45$ & $950(2)$ & $4615(2)$ & $6958.0(4)$ & $52.7(11)$ \\
\hline $\mathrm{C} 44$ & $1363.9(19)$ & $4824.7(18)$ & $6672.8(4)$ & $39.7(9)$ \\
\hline C55 & $2865(3)$ & $58(3)$ & $6215.0(7)$ & $34.2(8)$ \\
\hline C31 & $3854(3)$ & $7465(2)$ & $5853.2(7)$ & $25.2(6)$ \\
\hline C53 & $766(3)$ & $-19(2)$ & $6014.6(7)$ & $28.5(7)$ \\
\hline $\mathrm{C} 25$ & $4058(3)$ & $8245(2)$ & $6814.5(6)$ & $21.5(6)$ \\
\hline C60 & $586(3)$ & $3732(2)$ & $5332.3(6)$ & $19.5(6)$ \\
\hline C54 & $1808(3)$ & $-489(3)$ & $6147.6(7)$ & $33.8(8)$ \\
\hline C51 & 1883(3) & $1597(2)$ & $6011.9(6)$ & $20.8(6)$ \\
\hline $\mathrm{C} 17$ & $7031(3)$ & $12781(2)$ & $6067.9(7)$ & $22.6(6)$ \\
\hline $\mathrm{C} 18$ & $6515(3)$ & $13690(2)$ & $6190.1(7)$ & $26.2(6)$ \\
\hline $\mathrm{C} 28$ & $5851(3)$ & $8707(2)$ & $6390.6(6)$ & $19.5(6)$ \\
\hline $\mathrm{C} 22$ & $7542(3)$ & $12806(3)$ & $5758.0(7)$ & $27.0(7)$ \\
\hline C57 & $3360(3)$ & 2771(3) & $6099.3(7)$ & $25.2(6)$ \\
\hline C62 & $1856(3)$ & $4376(2)$ & $4943.9(6)$ & $22.2(6)$ \\
\hline $\mathrm{C} 30$ & $5406(3)$ & $6222(2)$ & $6086.8(7)$ & $26.2(6)$ \\
\hline C37 & $375.3(15)$ & $5494.9(14)$ & $5874.4(4)$ & $22.6(6)$ \\
\hline $\mathrm{C} 42$ & $-895.9(16)$ & $5358.4(14)$ & $5886.9(4)$ & $26.2(6)$ \\
\hline C41 & $-1659.1(13)$ & $5899.0(17)$ & $5694.2(5)$ & $33.1(7)$ \\
\hline $\mathrm{C} 40$ & $-1151.2(17)$ & $6576.2(16)$ & $5488.9(4)$ & $34.2(8)$ \\
\hline C39 & $120.0(19)$ & $6712.6(15)$ & $5476.3(4)$ & $34.2(8)$ \\
\hline C38 & $883.3(13)$ & $6172.0(16)$ & $5669.0(5)$ & $27.5(7)$ \\
\hline $\mathrm{C} 33$ & $3146(3)$ & $7947(3)$ & $7062.3(7)$ & $27.4(7)$ \\
\hline C64 & $2715(3)$ & $4086(2)$ & $5424.0(6)$ & $19.5(6)$ \\
\hline C49 & $1384(3)$ & $3485(2)$ & $5852.0(6)$ & $18.4(5)$ \\
\hline C69 & $-464(3)$ & $4066(2)$ & $4833.8(7)$ & $23.5(6)$ \\
\hline C71 & $-756(3)$ & $5178(3)$ & $4731.1(8)$ & $35.7(8)$ \\
\hline C61 & $682(3)$ & $4050(2)$ & $5037.5(6)$ & $20.9(6)$ \\
\hline C63 & $2884(3)$ & $4422(2)$ & $5133.7(6)$ & $20.3(6)$ \\
\hline $\mathrm{C} 21$ & $5898(3)$ & $11602(2)$ & $6987.6(7)$ & $28.1(7)$ \\
\hline $\mathrm{C} 14$ & $6469(3)$ & $11133(2)$ & $6753.4(6)$ & $20.9(6)$ \\
\hline $\mathrm{C} 23$ & $5826(3)$ & $9261(2)$ & $6653.3(6)$ & $19.0(6)$ \\
\hline $\mathrm{C} 1$ & $8073(2)$ & $8241.0(12)$ & $6933.8(5)$ & $27.3(7)$ \\
\hline C6 & 8888.9(19) & $7986.4(15)$ & $6707.7(5)$ & $34.6(8)$ \\
\hline $\mathrm{C} 5$ & $8958(2)$ & $6975.5(18)$ & $6606.2(5)$ & $46.4(10)$ \\
\hline $\mathrm{C} 4$ & $8211(2)$ & $6219.3(13)$ & $6730.9(6)$ & $52.1(11)$ \\
\hline $\mathrm{C} 3$ & $7395(2)$ & $6474.0(14)$ & $6957.0(6)$ & $44.8(10)$ \\
\hline $\mathrm{C} 2$ & $7326(2)$ & $7484.8(17)$ & $7058.4(5)$ & $36.1(8)$ \\
\hline
\end{tabular}




\begin{tabular}{|c|c|c|c|c|}
\hline $\mathrm{C} 32$ & $6081(3)$ & $7805(2)$ & $5827.4(7)$ & $25.0(6)$ \\
\hline C66 & $5072(3)$ & $4889(3)$ & $5284.4(7)$ & $30.6(7)$ \\
\hline C65 & $4130(3)$ & $4861(2)$ & $5031.5(6)$ & $23.3(6)$ \\
\hline $\mathrm{C} 70$ & $-294(3)$ & $3354(3)$ & $4562.6(7)$ & $31.7(7)$ \\
\hline C59 & $1575(3)$ & $3740(2)$ & $5526.5(6)$ & $18.1(5)$ \\
\hline $\mathrm{C} 13$ & $6850(3)$ & $10029(2)$ & $6718.0(6)$ & $20.3(6)$ \\
\hline $\mathrm{C} 72$ & $-1627(3)$ & $3683(3)$ & 4993.3(7) & $29.2(7)$ \\
\hline C68 & $4689(3)$ & $4198(3)$ & $4783.5(7)$ & $29.2(7)$ \\
\hline $\mathrm{C} 7$ & $9187.1(16)$ & 10309.9(17) & $7027.0(4)$ & $24.8(6)$ \\
\hline $\mathrm{C} 12$ & $9648(2)$ & $10606(2)$ & $7301.4(3)$ & $50.5(11)$ \\
\hline $\mathrm{C} 11$ & $10745(2)$ & $11158(2)$ & $7318.8(4)$ & $64.2(15)$ \\
\hline $\mathrm{C} 10$ & $11381.2(18)$ & $11415(2)$ & $7061.6(5)$ & $40.5(9)$ \\
\hline C9 & 10919.9(19) & $11118.7(19)$ & $6787.2(4)$ & $33.6(7)$ \\
\hline $\mathrm{C} 8$ & $9822.9(19)$ & $10566.3(18)$ & $6769.8(3)$ & $28.8(7)$ \\
\hline $\mathrm{C} 24$ & $4905(3)$ & $9034(2)$ & $6859.9(6)$ & $21.1(6)$ \\
\hline C34 & $3432(3)$ & $6845(3)$ & $7167.7(8)$ & $36.0(8)$ \\
\hline C36 & $1810(3)$ & $8031(3)$ & $6953.6(8)$ & $35.7(8)$ \\
\hline $\mathrm{C} 35$ & $3270(3)$ & $8665(3)$ & $7332.3(7)$ & $38.7(9)$ \\
\hline C58 & $-370(4)$ & $-648(3)$ & $5947.8(8)$ & $38.7(9)$ \\
\hline C67 & $3959(3)$ & $5981(3)$ & $4926.7(8)$ & $34.2(8)$ \\
\hline O5 & $6171(4)$ & $8836(4)$ & $7813.6(7)$ & $95.4(16)$ \\
\hline
\end{tabular}

\section{Bond lengths $\left[\mathrm{A}^{\circ}\right]$ for $3 \mathrm{z}$.}

\begin{tabular}{|c|c|c|c|}
\hline Atom-Atom & Length/ $\mathbf{A}^{0}$ & Atom-Atom & Length/ $\mathbf{A}^{0}$ \\
\hline P2-O3 & $1.487(2)$ & $\mathrm{C} 25-\mathrm{C} 33$ & $1.542(4)$ \\
\hline P2-C37 & $1.8066(15)$ & C60-C59 & $1.385(4)$ \\
\hline P2-C43 & $1.8092(16)$ & C60-C61 & $1.397(4)$ \\
\hline P2-C49 & $1.837(3)$ & C17-C18 & $1.415(4)$ \\
\hline P1-O1 & $1.489(2)$ & $\mathrm{C} 17-\mathrm{C} 22$ & $1.505(4)$ \\
\hline $\mathrm{P} 1-\mathrm{C} 1$ & $1.8138(18)$ & $\mathrm{C} 28-\mathrm{C} 23$ & $1.387(4)$ \\
\hline $\mathrm{P} 1-\mathrm{C} 7$ & $1.8134(17)$ & C62-C63 & $1.406(4)$ \\
\hline P1-C13 & $1.832(3)$ & C62-C61 & $1.406(4)$ \\
\hline $\mathrm{O} 2-\mathrm{C} 26$ & $1.369(3)$ & C37-C42 & 1.3900 \\
\hline O4-C62 & $1.388(3)$ & C37-C38 & 1.3900 \\
\hline $\mathrm{N} 1-\mathrm{C} 21$ & $1.370(4)$ & C42-C41 & 1.3900 \\
\hline $\mathrm{N} 1-\mathrm{C} 20$ & $1.374(4)$ & $\mathrm{C} 41-\mathrm{C} 40$ & 1.3900 \\
\hline N2-C57 & $1.371(4)$ & C40-C39 & 1.3900 \\
\hline
\end{tabular}




$\begin{array}{llll}\text { N2-C56 } & 1.378(4) & \text { C39-C38 } & 1.3900 \\ \text { C27-C28 } & 1.390(4) & \text { C33-C36 } & 1.532(5) \\ \text { C27-C26 } & 1.412(4) & \text { C33-C34 } & 1.537(5) \\ \text { C27-C29 } & 1.536(4) & \text { C33-C35 } & 1.539(5) \\ \text { C20-C19 } & 1.385(4) & \text { C64-C63 } & 1.393(4) \\ \text { C20-C15 } & 1.419(4) & \text { C64C59 } & 1.393(4) \\ \text { C29-C32 } & 1.536(4) & \text { C49-C59 } & 1.520(4) \\ \text { C29-C30 } & 1.541(4) & \text { C69-C72 } & 1.534(4) \\ \text { C29-C31 } & 1.546(4) & \text { C69-C61 } & 1.545(4) \\ \text { C56-C55 } & 1.377(5) & \text { C69C71 } & 1.546(4) \\ \text { C56-C51 } & 1.409(4) & \text { C69-C70 } & 1.544(4) \\ \text { C26-C25 } & 1.412(4) & \text { C63-C65 } & 1.537(4) \\ \text { C50-C57 } & 1.367(4) & \text { C21-C14 } & 1.367(4) \\ \text { C50-C51 } & 1.436(4) & \text { C14-C13 } & 1.498(4) \\ \text { C50-C49 } & 1.503(4) & \text { C23-C24 } & 1.397(4) \\ \text { C19-C18 } & 1.385(4) & \text { C23-C13 } & 1.519(4) \\ \text { C52-C53 } & 1.385(4) & \text { C1-C6 } & 1.3900 \\ \text { C52-C51 } & 1.405(4) & \text { C1-C2 } & 1.3900 \\ \text { C15-C16 } & 1.411(4) & \text { C6-C5 } & 1.3900 \\ \text { C15-C14 } & 1.430(4) & \text { C5-C4 } & 1.3900 \\ \text { C16-C17 } & 1.381(4) & \text { C4-C3 } & 1.3900 \\ \text { C43-C48 } & 1.3900 & \text { C3-C2 } & 1.3900 \\ \text { C43-C44 } & 1.3900 & \text { C66-C65 } & 1.532(4) \\ \text { C48-C47 } & 1.3900 & \text { C65-C68 } & 1.536(4) \\ \text { C47-C46 } & 1.3900 & \text { C65-C67 } & 1.538(4) \\ \text { C46-C45 } & 1.3900 & \text { C7-C12 } & 1.3900 \\ \text { C45-C44 } & 1.3900 & \text { C7-C8 } & 1.3900 \\ \text { C55-C54 } & 1.381(5) & \text { C12-C11 } & 1.3900 \\ \text { C53-C54 } & 1.417(5) & \text { C11-C10 } & 1.3900 \\ \text { C53-C58 } & 1.507(5) & \text { C10-C9 } & 1.3900 \\ \text { C25-C24 } & 1.390(4) & \text { C9-C8 } & 1.3900\end{array}$

Symmetry transformations used to generate equivalent atoms:

\section{Bond angles $\left[{ }^{\circ}\right]$ for $3 z$.}




\begin{tabular}{|c|c|c|c|}
\hline Atom-Atom-Atom & Angle $/^{\circ}$ & Atom-Atom-Atom & Angle ${ }^{\circ}$ \\
\hline O3-P2-C37 & $111.27(11)$ & O4-C62-C61 & $120.9(3)$ \\
\hline O3-P2-C43 & $112.11(11)$ & C63-C62-C61 & $123.1(3)$ \\
\hline C37-P2-C43 & $109.76(10)$ & C42-C37-C38 & 120.0 \\
\hline O3-P2-C49 & $115.32(13)$ & C42-C37-P2 & $122.60(11)$ \\
\hline C37-P2-C49 & $101.76(11)$ & C38-C37-P2 & $116.63(11)$ \\
\hline C43-P2-C49 & $106.00(11)$ & C37-C42-C41 & 120.0 \\
\hline O1-P1-C1 & $112.27(12)$ & $\mathrm{C} 40-\mathrm{C} 41-\mathrm{C} 42$ & 120.0 \\
\hline O1-P1-C7 & $111.60(12)$ & C41-C40-C39 & 120.0 \\
\hline C1-P1-C7 & $109.97(11)$ & C40-C39-C38 & 120.0 \\
\hline O1-P1-C13 & $113.89(13)$ & C39-C38-C37 & 120.0 \\
\hline C1-P1-C13 & $102.44(12)$ & C36-C33-C34 & $110.8(3)$ \\
\hline C7-P1-C13 & $106.13(12)$ & C36-C33-C35 & $107.1(3)$ \\
\hline C21-N1-C20 & $109.3(2)$ & C34-C33-C35 & $107.4(3)$ \\
\hline C57-N2-C56 & $109.5(3)$ & C36-C33-C25 & $110.9(3)$ \\
\hline C28-C27-C26 & $117.5(3)$ & C34-C33-C25 & 109.1(3) \\
\hline C28-C27-C29 & $120.0(3)$ & C35-C33-C25 & $111.5(3)$ \\
\hline C26-C27-C29 & $122.4(3)$ & C63-C64-C59 & $122.0(3)$ \\
\hline N1-C20-C19 & $131.4(3)$ & C50-C49-C59 & $117.0(2)$ \\
\hline N1-C20-C15 & $106.9(3)$ & C50-C49-P2 & $111.00(19)$ \\
\hline C19-C20-C15 & $121.7(3)$ & C59-C49-P2 & 109.62(19) \\
\hline C27-C29-C32 & $111.7(2)$ & C72-C69-C61 & $112.1(2)$ \\
\hline C27-C29-C30 & $109.5(2)$ & C72-C69-C71 & $105.9(3)$ \\
\hline C32-C29-C30 & $107.0(2)$ & C61-C69-C71 & $110.8(3)$ \\
\hline C27-C29-C31 & $111.1(2)$ & C72-C69-C70 & $106.1(3)$ \\
\hline C32-C29-C31 & $105.7(2)$ & C61-C69-C70 & $111.6(3)$ \\
\hline C30-C29-C31 & $111.8(2)$ & C71-C69-C70 & $110.1(3)$ \\
\hline C55-C56-N2 & $130.8(3)$ & C60-C61-C62 & $116.2(3)$ \\
\hline C55-C56-C51 & $122.3(3)$ & C60-C61-C69 & $120.7(3)$ \\
\hline N2-C56-C51 & $106.8(3)$ & C62-C61-C69 & $123.0(3)$ \\
\hline $\mathrm{O} 2-\mathrm{C} 26-\mathrm{C} 25$ & $115.8(3)$ & C64-C63-C62 & $117.1(3)$ \\
\hline $\mathrm{O} 2-\mathrm{C} 26-\mathrm{C} 27$ & $122.8(3)$ & C64-C63-C65 & $120.9(3)$ \\
\hline $\mathrm{C} 25-\mathrm{C} 26-\mathrm{C} 27$ & $121.5(3)$ & C62-C63-C65 & $121.9(3)$ \\
\hline C57-C50-C51 & $106.4(3)$ & C14-C21-N1 & $110.2(3)$ \\
\hline C57-C50-C49 & $128.6(3)$ & C21-C14-C15 & 106.3(3) \\
\hline C51-C50-C49 & $125.1(3)$ & C21-C14-C13 & $129.2(3)$ \\
\hline
\end{tabular}




\begin{tabular}{|c|c|c|c|}
\hline C18-C19-C20 & $117.7(3)$ & C15-C14-C13 & $124.4(3)$ \\
\hline C53-C52-C51 & $120.2(3)$ & $\mathrm{C} 28-\mathrm{C} 23-\mathrm{C} 24$ & $118.4(3)$ \\
\hline C16-C15-C20 & 119.0(3) & C28-C23-C13 & $119.3(3)$ \\
\hline C16-C15-C14 & $133.6(3)$ & $\mathrm{C} 24-\mathrm{C} 23-\mathrm{C} 13$ & $122.1(3)$ \\
\hline C20-C15-C14 & 107.4(2) & $\mathrm{C} 6-\mathrm{C} 1-\mathrm{C} 2$ & 120.0 \\
\hline C17-C16-C15 & $120.0(3)$ & C6-C1-P1 & 121.61(12) \\
\hline C48-C43-C44 & 120.0 & C2-C1-P1 & $117.64(12)$ \\
\hline $\mathrm{C} 48-\mathrm{C} 43-\mathrm{P} 2$ & $124.28(11)$ & $\mathrm{C} 5-\mathrm{C} 6-\mathrm{C} 1$ & 120.0 \\
\hline C44-C43-P2 & $115.72(11)$ & $\mathrm{C} 6-\mathrm{C} 5-\mathrm{C} 4$ & 120.0 \\
\hline $\mathrm{C} 43-\mathrm{C} 48-\mathrm{C} 47$ & 120.0 & $\mathrm{C} 5-\mathrm{C} 4-\mathrm{C} 3$ & 120.0 \\
\hline C46-C47-C48 & 120.0 & $\mathrm{C} 2-\mathrm{C} 3-\mathrm{C} 4$ & 120.0 \\
\hline C45-C46-C47 & 120.0 & $\mathrm{C} 3-\mathrm{C} 2-\mathrm{C} 1$ & 120.0 \\
\hline C44-C45-C46 & 120.0 & C66-C65-C63 & $111.8(2)$ \\
\hline C45-C44-C43 & 120.0 & C66-C65-C68 & $107.0(3)$ \\
\hline C56-C55-C54 & $117.8(3)$ & C63-C65-C68 & $111.0(2)$ \\
\hline C52-C53-C54 & 118.7(3) & C66-C65-C67 & $106.7(3)$ \\
\hline C52-C53-C58 & 121.1(3) & C63-C65-C67 & 109.6(3) \\
\hline C54-C53-C58 & $120.2(3)$ & C68-C65-C67 & $110.6(3)$ \\
\hline C24-C25-C26 & 118.0(3) & C60-C59-C64 & $118.6(3)$ \\
\hline $\mathrm{C} 24-\mathrm{C} 25-\mathrm{C} 33$ & $120.0(3)$ & C60-C59-C49 & $120.3(2)$ \\
\hline C26-C25-C 33 & $121.9(3)$ & C64-C59-C49 & $120.8(2)$ \\
\hline C59-C60-C61 & 122.9(3) & C14-C13-C23 & 116.4(2) \\
\hline C55-C54-C53 & $122.2(3)$ & C14-C13-P1 & $112.1(2)$ \\
\hline C52-C5-C56 & 118.7(3) & C23-C13-P1 & $109.46(19)$ \\
\hline C52-C51-C50 & $133.7(3)$ & $\mathrm{C} 12-\mathrm{C} 7-\mathrm{C} 8$ & 120.0 \\
\hline C56-C51-C50 & $107.5(3)$ & C12-C7-P1 & $115.96(12)$ \\
\hline C16-C17-C18 & 119.1(3) & C8-C7-P1 & $124.04(12)$ \\
\hline C16-C17-C22 & 121.5(3) & C7-C12-C11 & 120.0 \\
\hline C18-C17-C22 & $119.3(3)$ & $\mathrm{C} 10-\mathrm{C} 11-\mathrm{C} 12$ & 120.0 \\
\hline C19-C18-C17 & $122.4(3)$ & C11-C10-C9 & 120.0 \\
\hline $\mathrm{C} 23-\mathrm{C} 28-\mathrm{C} 27$ & $122.6(3)$ & C8-C9-C10 & 120.0 \\
\hline C50-C57-N2 & $109.9(3)$ & $\mathrm{C} 9-\mathrm{C} 8-\mathrm{C} 7$ & 120.0 \\
\hline O4-C62-C63 & 116.0(3) & C25-C24-C23 & 121.9(3) \\
\hline
\end{tabular}

Symmetry transformations used to generate equivalent atoms: 
displacement factor exponent takes the form: $-2 p^{2}\left[h^{2} a^{* 2} U^{11}+\ldots+2 h k a^{*} b^{*} U^{12}\right]$

\begin{tabular}{|c|c|c|c|c|c|c|}
\hline Atom & $\mathrm{U}^{11}$ & $\mathrm{U}^{22}$ & $\mathrm{U}^{33}$ & $U^{23}$ & $\mathrm{U}^{13}$ & $\mathrm{U}^{12}$ \\
\hline $\mathrm{P} 2$ & $20.6(3)$ & $21.5(4)$ & $18.2(3)$ & $-0.4(3)$ & $0.5(3)$ & $-2.0(3)$ \\
\hline P1 & $24.2(4)$ & $23.6(4)$ & $18.5(3)$ & $2.5(3)$ & $-2.9(3)$ & $-1.1(3)$ \\
\hline $\mathrm{O} 2$ & $28.2(11)$ & $25.1(12)$ & $26.6(11)$ & $-0.8(9)$ & $2.1(9)$ & $-5.5(9)$ \\
\hline $\mathrm{O} 4$ & 29.9(11) & $38.0(13)$ & $17.2(10)$ & $7.5(9)$ & $0.3(9)$ & $-3.4(10)$ \\
\hline $\mathrm{O} 3$ & $23.4(10)$ & $30.1(12)$ & $24.2(11)$ & $-0.3(9)$ & $0.1(9)$ & $-6.2(9)$ \\
\hline $\mathrm{O} 1$ & $33.5(12)$ & $35.4(12)$ & $18.8(10)$ & $4.5(9)$ & $-0.1(9)$ & $-3.5(10)$ \\
\hline N1 & $36.9(15)$ & $24.0(14)$ & $22.7(13)$ & $-6.7(10)$ & $6.2(12)$ & $3.6(11)$ \\
\hline $\mathrm{N} 2$ & $21.5(13)$ & $42.5(16)$ & $23.4(13)$ & $2.8(12)$ & $-4.4(11)$ & $9.7(12)$ \\
\hline $\mathrm{C} 27$ & $22.3(14)$ & $16.9(13)$ & 18.1(13) & $2.0(11)$ & $-2.2(11)$ & $3.5(11)$ \\
\hline $\mathrm{C} 20$ & 21.1(14) & 19.2(14) & $25.4(15)$ & $-3.9(12)$ & $0.0(12)$ & $-0.5(11)$ \\
\hline $\mathrm{C} 29$ & 23.9(14) & $18.8(14)$ & $19.6(14)$ & $-1.0(11)$ & $-1.2(12)$ & $1.7(11)$ \\
\hline C56 & $28.1(15)$ & $32.4(17)$ & $16.6(14)$ & $0.6(12)$ & $3.3(12)$ & $9.1(13)$ \\
\hline $\mathrm{C} 26$ & 21.4(14) & $17.5(14)$ & $22.4(14)$ & $3.6(11)$ & $-3.2(12)$ & $-0.2(11)$ \\
\hline $\mathrm{C} 50$ & $21.7(13)$ & $26.3(15)$ & $13.0(12)$ & $-0.8(11)$ & $0.8(11)$ & $1.9(12)$ \\
\hline C19 & $26.8(15)$ & $17.7(15)$ & $35.1(17)$ & $-3.7(12)$ & $-0.8(13)$ & $2.6(12)$ \\
\hline C52 & $28.4(15)$ & $25.4(15)$ & $16.3(13)$ & $0.8(11)$ & $-0.4(12)$ & $4.6(13)$ \\
\hline $\mathrm{C} 15$ & 17.2(13) & 20.1(14) & $21.0(14)$ & $-3.5(11)$ & $-1.4(11)$ & $-0.5(11)$ \\
\hline $\mathrm{C} 16$ & $16.8(13)$ & 19.2(14) & $22.2(14)$ & $-0.9(11)$ & $-0.1(11)$ & $0.9(11)$ \\
\hline $\mathrm{C} 43$ & $24.5(14)$ & $25.1(15)$ & $18.2(13)$ & $-3.5(11)$ & $0.0(12)$ & $1.9(12)$ \\
\hline $\mathrm{C} 48$ & $27.0(15)$ & $23.7(15)$ & $22.4(14)$ & $-1.2(12)$ & $0.1(12)$ & $-1.8(12)$ \\
\hline $\mathrm{C} 47$ & $28.9(16)$ & $25.5(16)$ & $26.1(15)$ & $1.3(13)$ & $6.2(13)$ & $-1.2(13)$ \\
\hline $\mathrm{C} 46$ & $47(2)$ & $55(2)$ & $21.4(16)$ & $-7.1(16)$ & $11.6(16)$ & $-8.5(19)$ \\
\hline $\mathrm{C} 45$ & $55(2)$ & $80(3)$ & $23.6(17)$ & $-18.6(19)$ & $8.9(17)$ & $-26(2)$ \\
\hline $\mathrm{C} 44$ & 41.8(19) & $51(2)$ & $26.5(16)$ & $-13.3(16)$ & $6.9(15)$ & $-17.8(18)$ \\
\hline C55 & 41.3(19) & $35.5(19)$ & $25.7(16)$ & $7.4(14)$ & $2.5(15)$ & $19.8(16)$ \\
\hline $\mathrm{C} 31$ & $27.8(15)$ & $26.4(16)$ & $21.5(15)$ & $-1.9(12)$ & $-3.9(12)$ & $0.6(13)$ \\
\hline $\mathrm{C} 53$ & $42.9(19)$ & $22.5(15)$ & $20.1(15)$ & $-1.0(11)$ & $3.7(14)$ & $3.0(14)$ \\
\hline $\mathrm{C} 25$ & 19.1(13) & $25.3(15)$ & $20.1(14)$ & $4.9(11)$ & $-1.5(11)$ & $1.0(12)$ \\
\hline C60 & $20.3(13)$ & $19.0(14)$ & $19.2(13)$ & $-0.5(11)$ & $0.5(11)$ & $-0.3(11)$ \\
\hline C54 & $51(2)$ & $23.5(17)$ & $26.4(16)$ & $4.3(13)$ & $5.6(15)$ & $12.1(15)$ \\
\hline $\mathrm{C} 51$ & $24.7(14)$ & $23.9(15)$ & $13.7(12)$ & $0.7(11)$ & $1.6(11)$ & $6.4(11)$ \\
\hline
\end{tabular}




\begin{tabular}{|c|c|c|c|c|c|c|}
\hline $\mathrm{C} 17$ & $18.7(13)$ & $24.6(15)$ & $24.4(14)$ & $3.9(12)$ & $-0.2(12)$ & $-0.8(11)$ \\
\hline C18 & $23.8(15)$ & $22.0(15)$ & $32.8(16)$ & $5.0(13)$ & $0.4(13)$ & $0.0(12)$ \\
\hline $\mathrm{C} 28$ & $21.4(13)$ & $18.6(14)$ & $18.5(13)$ & $2.8(11)$ & $-0.7(11)$ & $3.0(11)$ \\
\hline $\mathrm{C} 22$ & $25.3(16)$ & $28.5(16)$ & $27.0(16)$ & $6.7(13)$ & $3.8(12)$ & $-1.3(13)$ \\
\hline 57 & $22.6(14)$ & $33.1(17)$ & $19.8(14)$ & $-0.6(13)$ & $-0.2(12)$ & $3.7(12)$ \\
\hline C62 & $28.2(15)$ & $23.7(15)$ & $14.6(13)$ & $2.6(11)$ & $1.4(11)$ & $0.5(12)$ \\
\hline 30 & $28.9(15)$ & $22.3(15)$ & $27.4(15)$ & $-1.6(13)$ & $-3.7(13)$ & $2.5(12)$ \\
\hline C37 & $26.0(14)$ & $19.0(14)$ & $23.0(14)$ & $-2.4(11)$ & $-0.5(12)$ & $-0.5(12)$ \\
\hline 42 & $26.2(14)$ & $22.2(15)$ & $30.2(15)$ & $1.0(13)$ & $3.4(13)$ & $3.8(13)$ \\
\hline $\mathrm{C} 41$ & $27.7(16)$ & $33.1(18)$ & $38.4(18)$ & $0.8(15)$ & $3.7(14)$ & $8.4(14)$ \\
\hline $\mathrm{C} 40$ & $36.4(18)$ & $27.6(17)$ & $38.6(19)$ & $4.9(14)$ & $-2.7(15)$ & $12.6(14)$ \\
\hline C39 & $41.8(19)$ & $21.7(16)$ & 39.1(19) & $9.9(14)$ & $3.1(16)$ & $3.3(14)$ \\
\hline C38 & $29.8(16)$ & $23.4(16)$ & $29.4(16)$ & $3.0(12)$ & $-0.5(13)$ & $-2.5(13)$ \\
\hline $\mathrm{C} 33$ & $26.7(15)$ & $33.3(17)$ & $22.2(15)$ & $5.2(13)$ & $0.9(12)$ & $-2.2(13)$ \\
\hline C64 & $19.8(13)$ & $19.4(14)$ & $19.4(13)$ & $0.2(11)$ & $-1.1(11)$ & $-0.3(11)$ \\
\hline C49 & $17.7(13)$ & $20.8(14)$ & $16.7(13)$ & $-0.2(10)$ & $0.2(11)$ & $1.0(11)$ \\
\hline C69 & $22.6(14)$ & $30.1(17)$ & $17.9(14)$ & $4.1(12)$ & $-2.0(11)$ & $2.4(12)$ \\
\hline C71 & $36.6(18)$ & $40(2)$ & $30.6(17)$ & $11.0(15)$ & $-3.2(15)$ & $9.5(16)$ \\
\hline C61 & $23.5(14)$ & $20.4(14)$ & $18.8(13)$ & $0.0(11)$ & $-1.3(12)$ & $1.9(11)$ \\
\hline C63 & $21.0(13)$ & $18.2(14)$ & $21.7(14)$ & $-0.5(11)$ & $2.3(12)$ & $0.4(11)$ \\
\hline $\mathrm{C} 21$ & $39.3(18)$ & $26.1(16)$ & $18.9(14)$ & $-1.3(12)$ & $1.8(14)$ & $0.1(14)$ \\
\hline $\mathrm{C} 14$ & $23.1(14)$ & $20.8(14)$ & $18.7(13)$ & $-1.5(11)$ & $-0.8(12)$ & $1.3(12)$ \\
\hline $\mathrm{C} 23$ & $19.6(13)$ & $18.8(14)$ & $18.5(13)$ & $2.1(10)$ & $-2.3(11)$ & $2.8(11)$ \\
\hline $\mathrm{C} 1$ & $28.5(16)$ & $23.6(16)$ & $30.0(16)$ & $3.5(13)$ & $-8.6(13)$ & $1.4(12)$ \\
\hline C6 & $30.2(17)$ & $28.6(17)$ & $45(2)$ & $-5.8(15)$ & $-5.9(15)$ & $2.2(14)$ \\
\hline $\mathrm{C} 5$ & $40(2)$ & $37(2)$ & $62(3)$ & $-15.4(19)$ & $-5.3(19)$ & $8.1(17)$ \\
\hline $\mathrm{C} 4$ & $50(2)$ & $26(2)$ & $80(3)$ & $-8(2)$ & $-18(2)$ & $8.2(18)$ \\
\hline $\mathrm{C} 3$ & $49(2)$ & $25.8(18)$ & $59(3)$ & $7.6(17)$ & $-14(2)$ & $-4.3(16)$ \\
\hline $\mathrm{C} 2$ & $38.8(19)$ & $28.1(17)$ & $42(2)$ & $11.2(15)$ & $-9.1(16)$ & $-2.0(15)$ \\
\hline $\mathrm{C} 32$ & $26.9(15)$ & $27.9(16)$ & $20.3(14)$ & $-1.3(12)$ & $1.4(12)$ & $0.7(13)$ \\
\hline C66 & $26.3(16)$ & $39.5(19)$ & $26.0(16)$ & $-2.6(14)$ & $0.9(13)$ & $-7.8(14)$ \\
\hline C65 & $24.8(14)$ & $25.2(16)$ & $19.8(14)$ & $0.0(11)$ & $1.4(12)$ & $-4.4(12)$ \\
\hline $\mathrm{C} 70$ & $28.0(16)$ & $42(2)$ & $25.0(16)$ & $-0.4(14)$ & $-4.8(14)$ & $-1.5(15)$ \\
\hline C59 & $19.6(13)$ & $17.7(14)$ & $17.0(12)$ & $0.3(10)$ & $0.2(11)$ & $1.8(11)$ \\
\hline $\mathrm{C} 13$ & $23.5(14)$ & $20.9(14)$ & $16.5(13)$ & $0.0(11)$ & $1.1(11)$ & $0.2(11)$ \\
\hline $\mathrm{C} 72$ & $21.7(15)$ & $42.2(19)$ & $23.7(15)$ & $5.6(14)$ & $-4.0(12)$ & $0.5(14)$ \\
\hline
\end{tabular}




\begin{tabular}{lcccccc} 
C68 & $26.7(16)$ & $35.0(18)$ & $26.0(16)$ & $-1.6(13)$ & $6.5(13)$ & $-2.3(14)$ \\
C7 & $26.9(14)$ & $25.1(15)$ & $22.5(14)$ & $3.1(12)$ & $-4.1(12)$ & $-0.1(13)$ \\
C12 & $50(2)$ & $81(3)$ & $20.2(16)$ & $7.7(18)$ & $-6.0(16)$ & $-29(2)$ \\
C11 & $61(3)$ & $100(4)$ & $32(2)$ & $9(2)$ & $-17(2)$ & $-43(3)$ \\
C10 & $32.1(18)$ & $44(2)$ & $46(2)$ & $6.7(17)$ & $-7.0(17)$ & $-9.8(16)$ \\
C9 & $32.0(17)$ & $31.6(18)$ & $37.0(18)$ & $-0.2(15)$ & $7.5(15)$ & $-3.1(15)$ \\
C8 & $29.4(16)$ & $31.1(17)$ & $25.7(15)$ & $-4.7(13)$ & $1.6(13)$ & $-1.8(14)$ \\
C24 & $25.4(14)$ & $20.7(14)$ & $17.2(13)$ & $0.7(11)$ & $-1.7(12)$ & $2.5(12)$ \\
C34 & $37.8(19)$ & $42(2)$ & $27.9(17)$ & $12.0(15)$ & $1.6(15)$ & $-8.2(16)$ \\
C36 & $24.7(16)$ & $51(2)$ & $31.9(18)$ & $0.4(16)$ & $4.2(14)$ & $-0.4(15)$ \\
C35 & $37.4(19)$ & $56(2)$ & $22.5(16)$ & $-0.9(16)$ & $8.4(14)$ & $-8.1(17)$ \\
C58 & $58(2)$ & $26.1(18)$ & $32.3(18)$ & $1.4(14)$ & $2.2(17)$ & $-5.6(16)$ \\
C67 & $38.9(19)$ & $26.1(17)$ & $37.4(19)$ & $4.3(14)$ & $4.0(16)$ & $-8.7(15)$ \\
O5 & $112(3)$ & $138(4)$ & $36.8(17)$ & $29(2)$ & $-6(2)$ & $-85(3)$ \\
\hline
\end{tabular}

Hydrogen coordinates $\left(\mathrm{A}^{0} \times 10^{4}\right)$ and isotropic displacement parameters $\left(\mathrm{A}^{0^{2} \times 10^{3}}\right)$ for 3z.

\begin{tabular}{lcccl}
\hline Atom & $x$ & $y$ & $z$ & $U(e q)$ \\
\hline H2A & 3401.64 & 6611.2 & 6356.84 & 40 \\
H4 & 1428.59 & 4596.58 & 4556.18 & 43 \\
H1 & 5312.84 & 13050.18 & 7039.51 & 33 \\
H2 & 4490.9 & 1720.53 & 6274.27 & 35 \\
H19 & 5696.01 & 14328.35 & 6550.31 & 32 \\
H52 & 133.91 & 1348.11 & 5864.11 & 28 \\
H16 & 7431.63 & 11300.28 & 6162.45 & 23 \\
H48 & -533.51 & 3345.07 & 6313.33 & 29 \\
H47 & -1224.56 & 2995.78 & 6789.32 & 32 \\
H46 & -296.95 & 3790.17 & 7192.48 & 50 \\
H45 & 1321.7 & 4933.85 & 7119.64 & 63 \\
H44 & 2012.77 & 5283.15 & 6643.65 & 48 \\
H55 & 3537.44 & -262 & 6304.03 & 41 \\
H31A & 3181.68 & 7207.1 & 5970.31 & 38 \\
H31B & 3911.39 & 7075.46 & 5672.83 & 38 \\
H31C & 3711.46 & 8178.37 & 5807.05 & 38
\end{tabular}




\begin{tabular}{|c|c|c|c|}
\hline H60 & -175.34 & 3503.86 & 5401.14 \\
\hline H54 & 1779.33 & -1190.15 & 6190.98 \\
\hline H18 & 6499.97 & 14286.1 & 6075.5 \\
\hline $\mathrm{H} 28$ & 6463.49 & 8854.18 & 6252.63 \\
\hline $\mathrm{H} 22 \mathrm{~A}$ & 6957.94 & 13132.22 & 5628.36 \\
\hline $\mathrm{H} 22 \mathrm{~B}$ & 7693.51 & 12114.26 & 5691.53 \\
\hline $\mathrm{H} 22 \mathrm{C}$ & 8301 & 13188.44 & 5756.4 \\
\hline H57 & 3797.23 & 3387.1 & 6111.36 \\
\hline $\mathrm{H} 30 \mathrm{~A}$ & 6204.59 & 6183.85 & 6178.11 \\
\hline Н30B & 5419.36 & 5853.1 & 5902.32 \\
\hline H30C & 4801.9 & 5918.76 & 6215.83 \\
\hline H42 & -1235.69 & 4905.36 & 6024.29 \\
\hline H41 & -2509.61 & 5807.74 & 5702.6 \\
\hline $\mathrm{H} 40$ & -1661.86 & 6937.89 & 5359.93 \\
\hline H39 & 459.82 & 7165.67 & 5338.95 \\
\hline H38 & 1733.76 & 6263.3 & 5660.63 \\
\hline H64 & 3382.8 & 4092.38 & 5553.31 \\
\hline H49 & 537.69 & 3224.41 & 5869.05 \\
\hline H71A & -906.03 & 5605.38 & 4900.97 \\
\hline H71B & -1475.61 & 5171.69 & 4607.02 \\
\hline $\mathrm{H} 71 \mathrm{C}$ & -68.04 & 5446.23 & 4621.59 \\
\hline $\mathrm{H} 21$ & 5698.57 & 11279.8 & 7165.37 \\
\hline H6 & 9388.54 & 8492.31 & 6624.34 \\
\hline H5 & 9503.65 & 6805.15 & 6454.96 \\
\hline $\mathrm{H} 4 \mathrm{~A}$ & 8257.2 & 5543 & 6662.97 \\
\hline H3 & 6895.64 & 5968.01 & 7040.35 \\
\hline $\mathrm{H} 2 \mathrm{~B}$ & 6780.5 & 7655.17 & 7209.72 \\
\hline H32A & 5928.79 & 8525.35 & 5794.94 \\
\hline H32B & 6074.31 & 7447.97 & 5641.03 \\
\hline $\mathrm{H} 32 \mathrm{C}$ & 6871.54 & 7718.39 & 5920.23 \\
\hline H66A & 5225.95 & 4199.04 & 5352.74 \\
\hline H66B & 4752.96 & 5296.46 & 5444.49 \\
\hline H66C & 5828.8 & 5187.38 & 5214.44 \\
\hline H70A & -1052.18 & 3320.52 & 4453 \\
\hline H70B & -70.83 & 2674.8 & 4628.3 \\
\hline $\mathrm{H} 70 \mathrm{C}$ & 346.72 & 3624.18 & 4437.91 \\
\hline
\end{tabular}




$\begin{array}{lcclr}\text { H13 } & 7401.63 & 10010.16 & 6546.03 & 24 \\ \text { H72A } & -1788.36 & 4114 & 5161.83 & 44 \\ \text { H72B } & -1503.37 & 2984.77 & 5057.94 & 44 \\ \text { H72C } & -2316.51 & 3709.85 & 4860.05 & 44 \\ \text { H68A } & 5431.31 & 4517.86 & 4712.21 & 44 \\ \text { H68B } & 4108.1 & 4137.58 & 4624 & 44 \\ \text { H68C } & 4878.72 & 3524.49 & 4859.36 & 44 \\ \text { H12 } & 9223.04 & 10434.51 & 7473.45 & 61 \\ \text { H11 } & 11054.08 & 11356.49 & 7502.4 & 77 \\ \text { H10 } & 12115.23 & 11784.31 & 7073.25 & 49 \\ \text { H9 } & 11345.31 & 11290.17 & 6615.15 & 40 \\ \text { H8 } & 9514.25 & 10368.2 & 6586.19 & 35 \\ \text { H24 } & 4857.1 & 9422.78 & 7032.77 & 25 \\ \text { H34A } & 2892.44 & 6668.23 & 7328.49 & 54 \\ \text { H34B } & 4273.53 & 6808.69 & 7233.32 & 54 \\ \text { H34C } & 3308.99 & 6370.96 & 7007.05 & 54 \\ \text { H36A } & 1686.86 & 7569.09 & 6789.92 & 54 \\ \text { H36B } & 1647.52 & 8725.68 & 6891.05 & 54 \\ \text { H36C } & 1257.83 & 7850.12 & 7111.63 & 54 \\ \text { H35A } & 2709.85 & 8445.8 & 7484.62 & 58 \\ \text { H35B } & 3077.23 & 9359.32 & 7274.79 & 58 \\ \text { H35C } & 4100.33 & 8636.49 & 7405.97 & 58 \\ \text { H58A } & -1006.73 & -204.08 & 5872.74 & 58 \\ \text { H58B } & -652.02 & -977.39 & 6125.83 & 58 \\ \text { H58C } & -174.99 & -1162.79 & 5802.31 & 58 \\ \text { H67A } & 3620.67 & 6386.65 & 5085.14 & 51 \\ \text { H67B } & 3405.85 & 5995.51 & 4760.58 & 51 \\ \text { H67C } & 4742.95 & 6261.34 & 4868.97 & 51 \\ \text { H5A } & 6503.95 & 9099.25 & 7660.84 & 143 \\ \text { H5B } & 6664.41 & 8979.81 & 7954.1 & 143\end{array}$



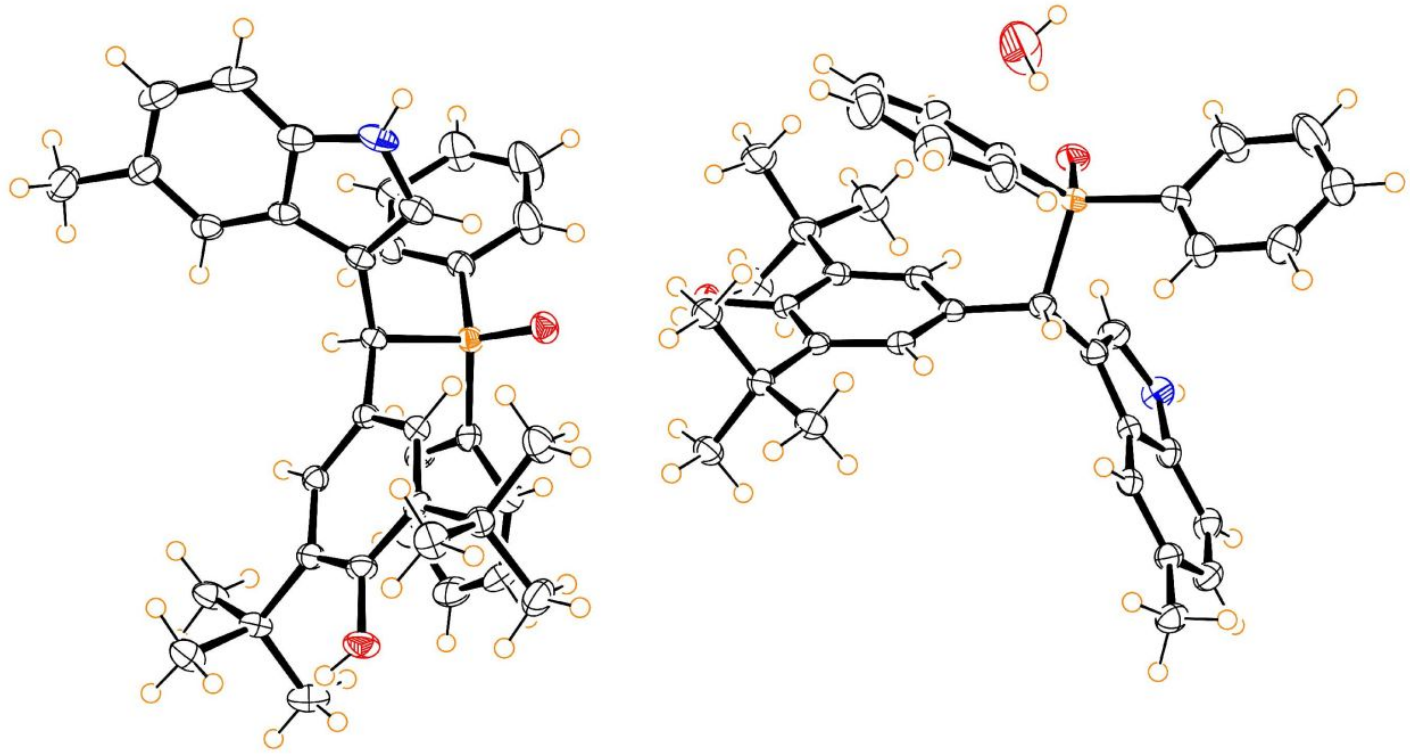

ORTEP representation of the $X$-ray structure of $3 z$ (thermal ellipsoids at $50 \%$ probability) 